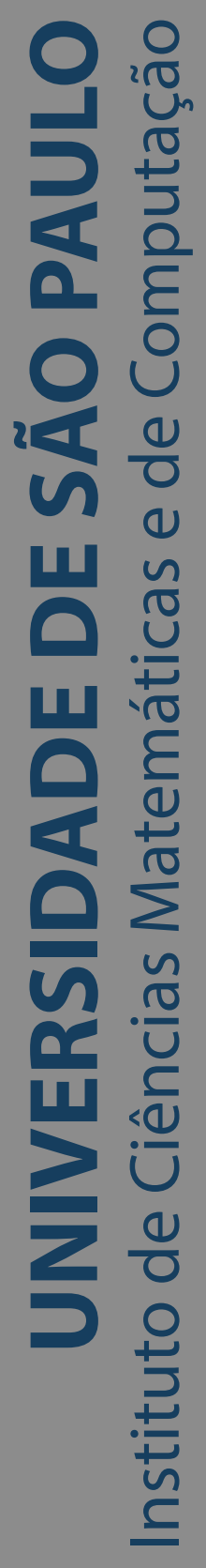

\title{
Bi-Lipschitz invariant geometry
}

\section{Thiago Filipe da Silva}

Doctoral Dissertation of the Graduate Program in Mathematics (PPG-Mat) 

Data de Depósito:

Assinatura:

\section{Thiago Filipe da Silva}

\section{Bi-Lipschitz invariant geometry}

Doctoral dissertation submitted to the Instituto de Ciências Matemáticas e de Computação - ICMC-USP, in partial fulfillment of the requirements for the degree of the Doctorate Program in Mathematics. FINAL VERSION

Concentration Area: Mathematics

Advisor: Prof. Dr. Nivaldo de Góes Grulha Júnior Co-advisor: Prof. Dr. Terence James Gaffney 
Ficha catalográfica elaborada pela Biblioteca Prof. Achille Bassi e Seção Técnica de Informática, ICMC/USP, com os dados inseridos pelo(a) autor(a)

da Silva, Thiago Filipe

Bi-Lipschitz invariant geometry / Thiago Filipe da Silva; orientador Nivaldo de Góes Grulha Júnior; coorientador Terence James Gaffney. -- São Carlos, 2018 .

$143 \mathrm{p}$.

Tese (Doutorado - Programa de Pós-Graduação em Matemática) -- Instituto de Ciências Matemáticas e de Computação, Universidade de São Paulo, 2018 .

1. Double of a module. 2. Lipschitz saturation of a module. 3. Bi-Lipschitz equisingularity. 4. Determinantal Varieties. 5. Integral closure of ideals and modules. I. de Góes Grulha Júnior, Nivaldo, orient. II. Gaffney, Terence James, coorient. III. Título. 


\section{Thiago Filipe da Silva}

\section{Geometria bi-Lipschitz invariante}

Tese apresentada ao Instituto de Ciências Matemáticas e de Computação - ICMC-USP, como parte dos requisitos para obtenção do título de Doutor em Ciências - Matemática. VERSÃO REVISADA

Área de Concentração: Matemática

Orientador: Prof. Dr. Nivaldo de Góes Grulha Júnior

Coorientador: Prof. Dr. Terence James Gaffney 

To Gesus, the OPrince of Oreace. 

Primeiramente agradeço a Deus, por Jesus, pela vida e pelo privilégio de aprender um pouco mais de Matemática.

Agradeço a minha esposa Mariana por todo o amor dedicado, pela compreensão nas horas difíceis e por ter feito parte deste tempo tão especial na minha vida. No início dessa jornada nós ainda éramos "apenas" namorados, separados por $1.140 \mathrm{~km}$, mas nada que o aeroporto de Viracopos e as promoções da Azul não pudessem dar um jeitinho na saudade. Depois fiz a melhor escolha da minha vida: após aquele período turbulento do exame de qualificação, eu casei com você. Te amo!

Agradeço meus pais Josué e Maria pelo amor, dedicação e orações. Foi muito bom ter vocês comigo aqui em São Carlos em 2014, com o "caçorro", o Lex! Agradeço minha irmã Jeane e ao meu cunhado Marcos pelas orações e pelas excelentes sugestões de boas músicas para ouvir durante o trabalho. Agradeço também minha irmã Samyra e ao meu cunhado José Antônio pelo carinho, por sempre nos receberem com tanto amor em sua casa em São Paulo. Foi um tempo precioso poder estar mais perto de vocês, da Clarice e Cecília, amores do titio. Além disso não poderia esquecer de agradecer a todos vocês especialmente pelo natal de 2014, onde tivemos a oportunidade de estarmos todos juntos comemorando o nascimento do nosso Salvador. Agradeço também minha segunda família em Vitória, Marlene e Luiz, pais da Mariana que me receberam tão carinhosamente em sua família, ao Higor, Heitor, a tia Carminha e a Laurinha, amor do titio.

Agradeço o Prof. Nivaldo, meu orientador, pelos valiosos momentos de pesquisa, pelo incentivo, por ter me dado a oportunidade de ter contato com outros grandes pesquisadores e especialmente pela grande amizade construída ao longo desses anos. Com certeza você, a Suelen e o Lucca tornaram nosso tempo aqui em São Carlos muito mais agradável e sentiremos muitas saudades.

I am very grateful to Professor Terence Gaffney, my co-advisor, for the valuable opportunity in work together. I am also grateful to Professor Gaffney and Mary Gaffney for the friendship and for the great support when I have been in Boston, for one year, for the care and kindness that you receive us.

Agradeço a Profa. Miriam, da UFPB, por ter nos recebido de forma tão carinhosa quando estivemos em João Pessoa em outubro e novembro de 2016. Foi um tempo muito proveitoso para a minha pesquisa poder trabalhar com a profa. Miriam, além de ter sido muito bom poder 
conhecer aquela cidade com um litoral tão lindo.

Agradeço ao Prof. Marcelo Escudero, da UEM, pela hospitalidade quando estivemos em Maringá em fevereiro de 2017. Foi um enorme prazer poder apresentar alguns pontos da minha pesquisa ao professor. Minha visita em Maringá com certeza me motivou bastante a continuar trabalhando, tendo em vista as palavras de incentivo que o professor Marcelo direcionou a mim. Além disso foi um grande prazer conhecer uma cidade tão linda como Maringá.

I am grateful to professor Anne Frühbis-Krüger for the valuable comments and suggestions in our work about Bi-Lipschitz equisingularity of Determinantal Surfaces.

I am grateful to the members of the board examination for the careful reading and the valuable suggestions which certainly improved this work.

Agradeço aos professores Behrooz, Irene, Ana Cláudia e Denise, meus professores nas disciplinas do programa, que tanto contribuíram para a minha formação.

Agradeço à professora Maria Aparecida Soares Ruas pelo incentivo e pelas valiosas conversas a respeito da minha pesquisa.

Agradeço a todos os professores do Departamento de Matemática da Universidade Federal do Espírito Santo pelo suporte e incentivo em realizar o doutorado. Em especial aos professores Leonardo e Alan, pelos conselhos e disposição em me auxiliar na escolha do ICMC como local de doutoramento, e ao professor Ricardo pelo grande auxílio com o fechamento das disciplinas sob minha responsabilidade antes do início do meu doutorado.

Agradeço especialmente aos meus amigos Maico, Fernando, Leandro, Telau, Karlo e Giovani, colegas da turma de 2009 do mestrado/UFES, pela força e companheirismo. Também agradeço a Jovane, Elivelton e Jhonatan, amigos muito especiais que apesar da distância, a amizade continua a mesma.

Agradeço aos irmãos da IPB Jardim Camburi, da IPB Central de São Carlos e da Segunda IPB de Nova Venécia pelas orações e acolhimento.

Agradeço o suporte financeiro da Fundação de Amparo à Pesquisa do Estado de São Paulo para a realização deste doutorado, processo no. 2013/22411-2, e pela Bolsa Estágio de Pesquisa no Exterior, processo no. 2015/09529-0. 
"Perhaps I could best describe my experience of doing mathematics in terms of entering a dark mansion. You go into the first room and it's dark, completely dark. You stumble around, bumping into the furniture. Gradually, you learn where each piece of furniture is. And finally, after six months or so, you find the light switch and turn it on. Suddenly, it's all illuminated and you can see exactly where you were. Then you enter the next dark room..." 



\section{ABSTRACT}

DA SILVA, T. F.. Bi-Lipschitz invariant geometry. 2018. 143 f. Doctoral dissertation (Doctorate Candidate Program in Mathematics) - Instituto de Ciências Matemáticas e de Computação (ICMC/USP), São Carlos - SP.

The study about bi-Lipschitz equisingularity has been a very important subject in Singularity Theory in last decades. Many different approach have cooperated for a better understanding about. One can see that the bi-Lipschitz geometry is able to detect large local changes in curvature more accurately than other kinds of equisingularity. The aim of this thesis is to investigate the bi-Lipschitz geometry in an algebraic viewpoint. We define some algebraic tools developing classical properties. From these tools, we obtain algebraic criterions for the bi-Lipschitz equisingularity of some families of analytic varieties. We present a categorical and homological viewpoints of these algebraic structure developed before. Finally, we approach algebraically the bi-Lipschitz equisingularity of a family of Essentially Isolated Determinantal Singularities.

Key-words: Double of a module, Lipschitz saturation of a module, bi-Lipschitz equisingularity, Determinantal varieties, Integral closure of ideals and modules. 



\section{RESUMO}

DA SILVA, T. F. $\quad$ Geometria bi-Lipschitz Invariante. 2017.143 p. $\quad$ Tese de doutorado (Candidato ao Programa de Doutorado em Matemática) - Instituto de Ciências Matemáticas e de Computação (ICMC/USP), São Carlos - SP.

$\mathrm{O}$ estudo da equisingularidade bi-Lipschitz tem sido amplamente investigado nas últimas décadas. Diversas abordagens têm contribuído para uma melhor compreensão a respeito. Observa-se que a geometria bi-Lipschitz é capaz de detectar grandes alterações locais de curvatura com maior precisão quando comparada a outros padrões de equisingularidade. $\mathrm{O}$ objetivo desta tese é investigar a geometria bi-Lipschitz do ponto de vista algébrico. Definimos algumas estruturas algébricas desenvolvendo algumas propriedades clássicas. A partir de tais estruturas obtemos critérios algébricos para a equisingularidade bi-Lipschitz de algumas classes de famílias de variedades analíticas. Apresentamos uma visão categórica e homológica dos elementos desenvol- vidos. Finalmente abordamos algebricamente a equisingularidade de famílias de Singularidades Determinantais Essencialmente Isoladas.

Palavras-chave: O double de um módulo, Saturação Lipschitz de um módulo, Equisingularidade biLipschitz, Variedades Determinantais, Fecho Integral de ideais e módulos. 

$\overline{-}$

Four moving lines . . . . . . . . . . . . . . . . . . 37

2

$X: y^{2} z^{2}=z^{3}+x^{2} \ldots \ldots \ldots \ldots \ldots \ldots \ldots$

3

Cone .............................. 48

4

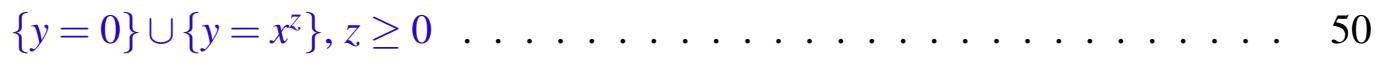

5

Cusp and Node . . . . . . . . . . . . . . . . . 50 50

6

Family of Cusps . . . . . . . . . . . . . . . . . 67 

1 Possible candidates of 1 -jets with 4 or more variables . . . . . . . . . 104

2 Simple isolated Cohen-Macaulay in codimension 2 singularities in $\left(\mathbb{C}^{4}, 0\right)$. 106

3 Simple isolated Cohen-Macaulay in codimension 2 singularities in $\left(\mathbb{C}^{5}, 0\right)$. . 107 

$\mathbb{Z}$ - Integer numbers

$\mathbb{C}$ - Complex numbers

$\mathbb{R}$ - Real numbers

$\mathbb{K}$ - The field $\mathbb{R}$ or $\mathbb{C}$

$\mathbb{P}^{N}$ - N-dimensional complex projective space

$\mathscr{O}_{X}$ - Analytic sheaf of rings of the analytic variety $X$

$\Delta(X)$ - Diagonal of the set $X$ in $X \times X$

$\ell(M)$ - The length of a module $M$

$\bar{M}$ - Integral closure of $M$

$\mathscr{R}(M)$ - Rees algebra of the module $M$

$I_{S}$ - Lipschitz saturation of the ideal $I$

$B_{I}(X)-$ Blow-up of $X$ with respect to the ideal $I$

$M_{D}$ - Double of the module $M$

$\operatorname{Proj}(G)$ - The projective spectrum of a graded ring $G$

$e(M)$ - The Buchsbaum-Rim multiplicity of a module $M$

$e(M, N)$ - The multiplicity of the pair of modules $(M, N)$

$\mathscr{F}_{x}$ - The stalk of the sheaf $\mathscr{F}$ at the point $x$

$\operatorname{supp}(\mathscr{F})$ - The support of the sheaf $\mathscr{F}$

$\operatorname{cosupp}(\mathscr{F})$ - The cosupport of the sheaf $\mathscr{F}$ 



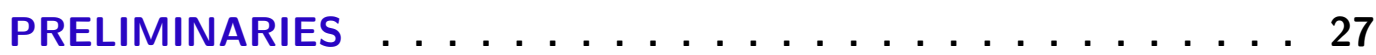

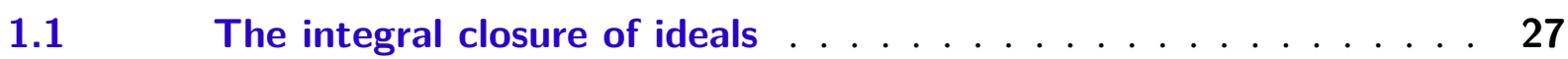

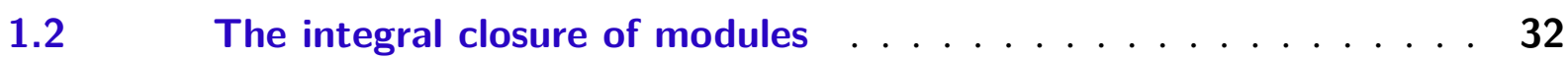

1.3 Whitney equisingularity and the integral closure of modules . . . . 36

1.4 The Lipschitz saturation and the double of an ideal . . . . . . 40

1.5 Determinantal Varieties . . . . . . . . . . . . . 44

$1.6 \quad$ Bi-Lipschitz geometry and Lipschitz stratifications . . . . . . 46

2 THE DOUBLE Of A MODULE $\ldots \ldots \ldots \ldots \ldots$

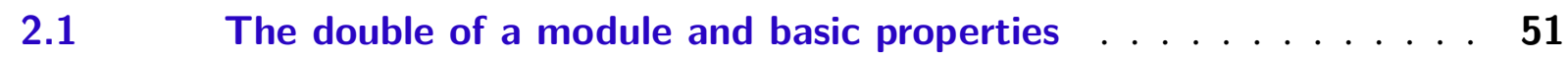

$2.2 \quad$ The infinitesimal Lipschitz conditions $i L_{A}$ and $i L_{m_{Y}} \ldots \ldots \ldots 6$

2.3 The genericity theorem applied in a family of hyperplane sections . 76

3 THE LIPSCHITZ SATURATION OF A MODULE . . . . . . . 81

3.1 The Lipschitz saturation of a module and basic properties . . . . . 81

3.2 The generic equivalence among the Lipschitz saturations . . . . . 93

$3.3 \quad$ Geometric applications . . . . . . . . . . . . . . . 99

4 BI-LIPSCHITZ EQUISINGULARITY OF DETERMINANTAL SUR-

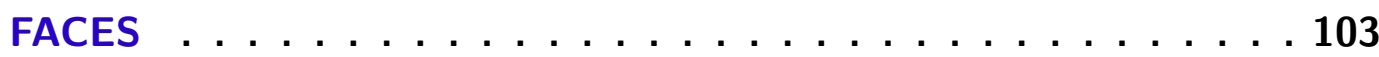

4.1 Simple isolated Cohen-Macaulay of codimension 2 singularities in $\mathbb{C}^{4}$ and $\mathbb{C}^{5} \ldots \ldots \ldots \ldots \ldots . \ldots \ldots 10 \ldots$

4.2 The bi-Lipschitz equisingularity on determinantal varieties . . . . 108

5 CATEGORICAL ASPECTS OF THE DOUBLE STRUCTURE $\ldots 113$

5.1 The double homomorphism and basic properties . . . . . . 113

$5.2 \quad$ Homological aspects of the double structure . . . . . . . . 122

$5.3 \quad$ The Double category . . . . . . . . . . . . . . . 125

$5.4 \quad$ The double in a quotient of a free $\mathscr{O}_{X}$-module of finite rank $\ldots 126$

5.5 The double homomorphism relative to an analytic map germ . . . 127

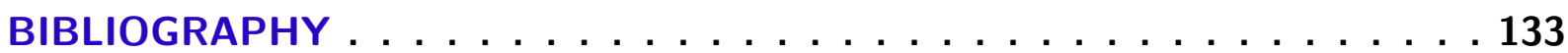

Index . . . . . . . . . . . . . . . . . . . 143 

The study about bi-Lipschitz equisingularity was started by Pham and Teissier [84, 85] at 1969. In these papers they defined the notion of the Lipschitz saturation of a local ring on the context of analytic complex algebras. After, in 1971 Zariski [111] developed the Lipschitz saturation of a complete local domain of dimension one. In the same year, [112] he defined the saturation of a local ring of dimension one, and in 1975 [113] he generalized this notion for local rings with arbitrary dimension. For more about Lipschitz saturation of local rings one can see the important contributions of Lipman $[65,66]$.

As reported by Trotman in [101], while Thom [99] was describing Whitney's work about stratifications of analytic varieties $[109,110]$ in 1964 at the Bourbaki seminar, he suggested that the vector fields yielding local topological triviality of Whitney stratifications (conjectured by Whitney in his contribution to the Morse Jubilee volume [109], although Whitney's conjecture, still unproven, required a much stronger notion of local triviality) might be made Lipschitz, hence the local bi-Lipschitz triviality would follow as a corollary. In his 1985 dissertation, Mostowski [72] proved the Thom's conjecture. Few years before in 1969, Pham and Teissier $[84,85]$ obtained a positive result for the special case of families of plane curves.

Using a set of equisingularity conditions, Mostowski [72] defined a notion of Lipschitz stratification (see the section 1.6) stronger than Verdier's $(w)$-regularity (see the section 1.3), and he proved that every complex analytic variety admits a Lipschitz stratification. Further, Mostowski proved that these Lipschitz stratifications are locally bi-Lipschitz trivial along strata by integrating stratified Lipschitz vector fields. Then, Parusiński generalized these theorems for every analytic real variety [78], after for all semianalytic sets [79], and finally in 1994 for every subanalytic set [82].

There are canonical Whitney (equivalently Verdier) stratifications of complex analytic varieties by the theory of Teissier [98] (see also [54]) which shows identifies canonical Whitney strata as precisely the loci of equimultiplicity of the different polar varieties. However, in general there is no canonical Lipschitz stratification attached to a given subanalytic set, even in the special cases of real algebraic varieties or complex algebraic varieties. For details, see Mostowski's habilitation dissertation [72]. Nevertheless, Mostowski [71] gave equivalent algebraic criteria for Lipschitz regularity in the case of complex surfaces and in [70] he also explained the close relation of the polar varieties of a complex analytic variety $X$ and the stratifications of $X$ with locally trivializing Lipschitz vector fields.

Many mathematicians worked or are still working on bi-Lipschitz geometry. We can 
cite, for instance, Birbrair [3, 4, 5, 6, 7, 8, 9, 10, 11, 12, 13, 14], Comte [20], Costa [22, 23, 24], Fernandes [6, 7, 8, 9, 10, 11, 12, 13, 32, 33, 34, 35], Gaffney [39, 40], Grandjean [8, 9], Grulha [26], Henry [55], Juniati [57, 58, 59], Kovalev [60], Lê [10], Mostowski [70, 71, 72, 73], Neumann [11, 12, 14, 74], O’Shea [9, 77], Parusiński [55, 78, 79, 80, 81, 82], Paunescu [61], Pham [84, 85], Pichon [74], Ruas [6, 33, 34, 91], Saia [23, 24], Sampaio [10, 35, 92], Teissier [85], Trotman [58, 59, 101], Valette [57, 59, 91, 102, 103, 104, 105, 106] and Zariski [111, 112, $113]$.

In [41], Gaffney used the integral closure of modules to describe the Whitney equisingularity. If $X \subseteq \mathbb{C}^{n} \times \mathbb{C}^{k}$ is a family of complex analytic varieties defined by an analytic map $F:\left(\mathbb{C}^{n} \times \mathbb{C}^{k}, 0\right) \rightarrow\left(\mathbb{C}^{p}, 0\right), Y=0 \times \mathbb{C}^{k} \equiv \mathbb{C}^{k} \subseteq X$ the parameter space and the singular locus of a small enough representative of $X$, Gaffney showed that $(X-Y, Y)$ is Whitney equisingular if and only if all the partial derivatives of $F$ with respect to the parameter space are in the integral closure of the submodule generated by $\left\{z_{i} \frac{\partial F}{\partial z_{j}}\right\}_{i, j=1}^{n}$, where $z_{1}, \ldots, z_{n}$ are the coordinate functions on $\mathbb{C}^{n}$. We denote this inclusion as

$$
J M(X)_{Y} \subseteq \overline{m_{Y} J M_{z}(X)}
$$

Since there is a close relation between the double structure and Lipschitz behavior, it is natural to hope that the above condition may desbribe the bi-Lipschitz equisingularity adding the double structure, i.e, $\left(J M(X)_{Y}\right)_{D} \subseteq \overline{\left(m_{Y} J M_{Z}(X)\right)_{D}}$, or even a weaker condition as $\left(J M(X)_{Y}\right)_{D} \subseteq$ $\overline{\left(J M_{z}(X)\right)_{D}}$. In the hypersurface case, Gaffney [39] called these the infinitesimal Lipschitz conditions $m_{Y}$ and A, respectively.

This thesis is divided into five chapters.

In the first chapter we first remember the main definitions and results about the integral closure of ideals and modules. Then we recall the Whitney and Verdier regularity conditions and its algebraic characterization obtained by Gaffney in [41] using the integral closure of modules. We see the definition of the Lipschitz saturation of an ideal, following the ideas of Pham, Teissier [85] and Zariski [111, 112, 113]. We also remember the main about the double of an ideal and the infinitesimal Lipschitz conditions for hypersurfaces given by Gaffney in [39, 40]. After we recall the definition of Determinantal Varieties, which will be used on the fourth chapter. We end the first chapter presenting the classical approach about bi-Lipschitz geometry due to Mostowski and Parusiński.

In the second chapter we start generalizing the concept of the double for modules, and we obtain several properties and results. Then we use the double structure to rephrase the infinitesimal Lipschitz conditions in any codimension, and we generalize some results of [39].

In the third chapter we define some different notions of Lipschitz saturations of a module, each one generalizing the original concept for ideals, and we get relations among them. At the end we apply the algebraic machinery developed to obtain conditions for bi-Lipschitz equisingularity of some families of analytic varieties. 
In the fourth chapter we investigate the bi-Lipschitz equisingularity for families of determinantal varieties. First we remember the classification of simple isolated singularities of codimension 2 in $\mathbb{C}^{4}$ and $\mathbb{C}^{5}$, given by Frühbis-Krüger and Neumer in [37]. Then we get a canonical stratified vector field defined on a semi-universal 1-unfolding of a determinantal variety which is Lipschitz when the double of the partial derivative of the unfolding with respect to the parameter belongs to the integral closure of the ideal generated by the double of the another partial derivatives. We also see that for the special case that the matrix of deformation is constant, the last above condition always is true and then the canonical vector field is Lipschitz.

Finally, in the fifth chapter we develop a categorical viewpoint of the double structure. We start defining the double of a homomorphism in a quite natural way. Then we look for homological properties of the double structure and we compare algebraic properties of the double homomorphism with the "single" homomorphism. We define the double category and we end the chapter generalizing the double homomorphism for two analytic varieties linked by an analytic map germ.

Have a good reading! 



\subsection{The integral closure of ideals}

In this section we present some classical results and definitions about the integral closure of an ideal following [95].

For more about this subject one can see the works of Bivià-Ausina [15, 16].

Definition 1.1.1. Let I be an ideal in a ring $R$. An element $h \in R$ is said to be integral over $\mathbf{I}$ if there exists a positive integer $n$ and elements $a_{i} \in I^{i}$, with $i \in\{1, \ldots, n\}$, such that

$$
h^{n}+a_{1} h^{n-1}+\ldots+a_{n-1} h+a_{n}=0 .
$$

Such an equation is called an equation of integral dependence of $\mathbf{h}$ over $\mathbf{I}$.

The set of all elements that are integral over $I$ is called the integral closure of $\mathbf{I}$, and is denoted by $\bar{I}$. If $\bar{I}=I$, then $I$ is called integrally closed. If $I \subseteq J$ are ideals, we say that $J$ is integral over $I$ if $J \subseteq \bar{I}$.

A basic example is the following:

Example 1.1.2. For arbitrary elements $x, y \in R, x y \in \overline{\left(x^{2}, y^{2}\right)}$. In fact, take $n=2, a_{1}=0 \in\left(x^{2}, y^{2}\right)$ and $a_{2}=-x^{2} y^{2} \in\left(x^{2}, y^{2}\right)^{2}$, notice that

$$
(x y)^{2}+a_{1}(x y)+a_{2}=0
$$

is an equation of integral dependence of $x y$ over $\left(x^{2}, y^{2}\right)$. Therefore, $(x, y)^{2}$ is integral over $\left(x^{2}, y^{2}\right)$.

Definition 1.1.3. Let $R \subseteq S$ be an extension of rings. An element $s \in S$ is called integral over $\boldsymbol{R}$ if $f(s)=0$ for some monic polynomial $f \in R[T]$. 
The next lemma is a link between the notions of integral closure of extensions of rings and ideals, and it is an important tool to prove that the integral closure of an ideal is also an ideal. Lemma 1.1.4. ([64] Lemma 1.3) Let $R$ be a ring and denote by $R[T]$ the ring of polynomials of one variable over $R$. Let $h \in R$ and I an ideal of $R$. Then: $h$ is integral over I if and only if $h T$ is integral over the subring $\bigoplus_{n \in \mathbb{Z}^{+}} I^{n} T^{n}$ of $R[T]$.

Now, using the previous lemma and the fact that the set of the elements which are integral over a subring is also a subring, we have the next

Proposition 1.1.5. ([64] Corollary 1.4) If I is an ideal of $R$ then $\bar{I}$ is an integrally closed ideal of $R$, i.e, $\overline{\bar{I}}=\bar{I}$.

Remark 1.1.6. ([95] Remark 1.1.3) Basic properties of the integral closure of ideals:

- $I \subseteq \bar{I}$, since for each $r \in I$, taking $n=1$ and $a_{1}=-r$ give an equation of integral dependence over I.

- If $I \subseteq J$ are ideals then $\bar{I} \subseteq \bar{J}$, since every equation of integral dependence of $r$ over I is also an equation of integral dependence of $r$ over $J$.

- $\bar{I} \subseteq \sqrt{I}$, since from the equation of integral dependence of some degree $n$ for $h \in \bar{I}$, we conclude that $h^{n} \in\left(a_{1}, \ldots, a_{n}\right) \subseteq I$.

- Radical, hence prime and maximal, ideals are integrally closed.

- The nilradical $\sqrt{0}$ of the ring is contained in I for every ideal I, because for each nilpotent element $r$ there exists an integer $n$ such that $r^{n}=0$ and this is an equation of integral dependence over $I$.

- Intersections of integrally closed ideals are integrally closed.

- The following property is called persistence: if $\varphi: R \longrightarrow S$ is a ring homomorphism, then $\varphi(\bar{I}) \subseteq \overline{\varphi(I) S}$. This follows as by applying $\varphi$ to an equation of integral dependence of an element $r$ over I to obtain an equation of integral dependence of $\varphi(r)$ over $\varphi(I) S$.

- Another important property is contraction: if $\varphi: R \longrightarrow S$ is a ring homomorphism and I is an integrally closed ideal of $S$, then $\varphi^{-1}(I)$ is integrally closed in $R$. In fact, if $r$ is integral over $\varphi^{-1}(I)$, then applying $\varphi$ to an equation of integral dependence of $r$ over $\varphi^{-1}(I)$ gives an equation of integral dependence of $\varphi(r)$ over $I$, hence $\varphi(r) \in I$ and $r \in \varphi^{-1}(I)$.

- In particular, if $R$ is a subring of $S$, and $I$ is an integrally closed ideal of $S$, then $I \cap R$ is an integrally closed ideal in $R$.

Integral closure behaves well under localization: 
Proposition 1.1.7. ([95] Proposition 1.1.4) Let $R$ be a ring and $I$ an ideal in $R$. For any multiplicatively closed subset $W$ of $R$,

$$
W^{-1} \bar{I}=\overline{W^{-1} I}
$$

Furthermore, the following are equivalent:

a) $\bar{I}=I$;

b) $\overline{W^{-1} I}=W^{-1} I$, for all multiplicatively closed subsets $W$ of $R$;

c) $\overline{I_{P}}=I_{P}$, for all prime ideals $P$ of $R$;

d) $\overline{I_{M}}=I_{M}$, for all maximal ideals $M$ of $R$.

The following proposition reduces questions about the integral closure to questions about integral closure in integral domains.

Proposition 1.1.8. ([95] Proposition 1.1.5) Let $R$ be a ring and I an ideal of $R$.

a) The image of the integral closure of I in $R_{\text {red }}$ is the integral closure of the image of I in $R_{\text {red }}: \bar{I} R_{\text {red }}=\overline{I R_{\text {red }}}$. Thus $\bar{I}$ equals the natural lift to $R$ of the integral closure of $I$ in the reduced ring $R_{\text {red }}$.

b) An element $r \in R$ is in the integral closure of I if and only if for every minimal prime ideal $P$ of $R$, the image of $r$ in $\frac{R}{P}$ is in the integral closure of $\frac{I+P}{P}$.

We next rephrase integral closure with ideal equalities:

Proposition 1.1.9. ([95] Proposition 1.1.7) Let $R$ be a ring, I an ideal of $R$ and $r \in R$. Then: $r \in \bar{I}$ if and only if there exists an integer $n$ such that $(I+(r))^{n}=I(I+(r))^{n-1}$.

One can also use modules to express integral dependence, usually known as determinantal trick.

Corollary 1.1.10. ([95] Corollary 1.1.8) Let I be an ideal of $R$ and $r \in R$. Then, the following are equivalent:

(a) $r$ is integral over I;

(b) There exists a finitely generated $R$-module $M$ such that $r M \subseteq I M$ and such that whenever $a M=0$ for some $a \in R$, then $a r \in \sqrt{0}$.

Moreover, if I is finitely generated and contains a non-zerodivisor, $r$ is integral over I if and only if there exists a finitely generated faithful $R$-module $M$ such that $I M=(I+(r)) M$. 
We introduce reductions, which are an extremely useful tool for integral closure in general.

Definition 1.1.11. Let $J \subseteq I$ be ideals in $R$. The ideal $J$ is said to be a reduction of I if there exists a non-negative integer $n$ such that $I^{n+1}=J I^{n}$.

As a consequence of Proposition 1.1.9 we have the following result.

Corollary 1.1.12. ([95] Corollary 1.2.2) An element $r \in R$ is integral over $J$ if and only if $J$ is a reduction of $J+(r)$.

Remark 1.1.13. Note that if $J I^{n}=I^{n+1}$, then for all positive integers $m, I^{m+n}=J I^{m+n-1}=$ $\ldots=J^{m} I^{n}$. In particular, if $J \subseteq I$ is a reduction, there exists an integer $n$ such that for all $m \geq 1$, $I^{m+n} \subseteq J^{m}$.

The reduction property is transitive:

Proposition 1.1.14. ([95] Proposition 1.2.4) Let $K \subseteq J \subseteq I$ be ideals in $R$.

a) If $K$ is a reduction of $J$ and $J$ is a reduction of $I$, then $K$ is a reduction of $I$;

b) If $K$ is a reduction of $I$ then $J$ is a reduction of $I$;

c) If I is finitely generated, $J=K+\left(r_{1}, \ldots, r_{k}\right)$, and $K$ is a reduction of $I$, then $K$ is a reduction of $J$.

The next corollary gives a relation between the integral closure and reductions.

Corollary 1.1.15. ([95] Corollary 1.2.5) Let $J \subseteq I$ be ideals in $R$. Suppose that I is finitely generated. Then $J$ is a reduction of $I$ if and only if $I \subseteq \bar{J}$.

In particular, if $R$ is a noetherian ring, then reduction property in $R$ is equivalent to the integral dependence.

Notice that in Example 1.1.2 we have seen that $\left(x^{2}, y^{2}\right)$ is a reduction of $(x, y)^{2}$.

The next result says about the behaviour of the integral closure with powers of an ideal.

Proposition 1.1.16. ([64] Corollary 1.8) Let I be an ideal of $R$.

a) $(\bar{I})^{n} \subseteq \overline{I^{n}}$;

b) $I \cdot \overline{I^{n}} \subseteq \overline{I^{n+1}}$.

As a corollary of the Corollary 1.1.10, we have the 
Corollary 1.1.17. ([64] Corollary 1.11) If I and $J$ are ideals in $R$ then

$$
\bar{I} \cdot \bar{J} \subseteq \overline{I \cdot J} .
$$

Proposition 1.1.18. ([64] Proposition 1.14) Let $R$ be an excellent reduced ring and I an ideal in $R$ containing a non-zerodivisor element of $R$. Then, there exists an integer $N$ such that, if $n \geq N$ :

a) $I \cdot \overline{I^{n}}=\overline{I^{n+1}}$;

b) $I .(\bar{I})^{n}=(\bar{I})^{n+1}$.

For the definition of excellent rings, see [52]. Every complete local ring is an excellent ring. For example, the local rings of the analytic geometry.

Notation 1.1.19. Let I be an ideal of $R$. We denote by e(I) the Hilbert-Samuel multiplicity of I.

Proposition 1.1.20. ([64] Proposition 1.18) Let $(R, m)$ be a local noetherian ring. If I and $J$ are $m$-primary ideals of $R$ with the same integral closure, i.e., $\bar{I}=\bar{J}$ then $e(I)=e(J)$.

For the definition of $m$-primary ideals and Hilbert-Samuel multiplicity see [31].

Remark 1.1.21. If $(R, m)$ is an analytic equidimensional local algebra, David Rees [86] showed a reciprocal: if $I$ and $J$ are $m$-primary ideals of $R$ such that $e(I)=e(J)$ and $I \subseteq J$ then $\bar{I}=\bar{J}$.

Let us recall the definition of nowhere dense in a topological space.

Definition 1.1.22. Let $X$ be a topological space. A subset $A \subseteq X$ is called nowhere dense if $\bar{A}$ has empty interior. Equivalently, a nowhere dense set is a set that is not dense in any non-empty open subset of $X$.

The next theorem is one of the most important result to our work. It gives several points of view of the integral closure of an ideal when we are working in analytic geometry.

Theorem 1.1.23. ([64] Theorem 2.1) Let $\left(X, \mathscr{O}_{X}\right)$ be a reduced complex analytic space, $x \in X$, and let $\mathscr{I}$ be a coherent sheaf of ideals of $\mathscr{O}_{X}$. Denote $I=\mathscr{I}_{x}$ the stalk of $\mathscr{I}$ on $x$, which is an ideal of $\mathscr{O}_{X, x}$. Let $h \in \mathscr{O}_{X, x}$. Suppose that $\mathscr{I}$ defines a nowhere dense closed subset of X. The following are equivalent:

(a) h is integral over I;

(b) There exist a neighborhood $U$ of $x$, a positive real number $C$, representatives of the space germ $X$, the function germ $h$, and generators $g_{1}, \ldots, g_{m}$ of I on $U$, which we identify with the corresponding germs, so that

$$
\|h(z)\| \leq C \max \left\{\left\|g_{1}(z)\right\|, \ldots,\left\|g_{m}(z)\right\|\right\},
$$

for all $z \in U$; 
(c) For all analytic path germs $\phi:(\mathbb{C}, 0) \rightarrow(X, x)$, the pullback $\phi^{*}(h)$ is contained in the ideal generated by $\phi^{*}(I)$ in the local ring $\mathscr{O}_{\mathbb{C}, 0}$;

(d) Let NB denote the normalization of the blowup of $X$ by $I, \bar{D}$ the pullback of the exceptional divisor of the blowup of $X$ by I. Then, for any irreducible component $C$ of the underlying set of $\bar{D}$, the order of vanishing of the pullback of $h$ to $N B$ along $C$ is greater than or equal to the order of the divisor $\bar{D}$ along $C$.

We end this section with a theorem that allows us work with the integral closure of a sheaf of ideals.

Theorem 1.1.24. ([64] Theorem 2.6) Let $\left(X, \mathscr{O}_{X}\right)$ be a reduced complex analytic space, and let $\mathscr{I}$ be a coherent sheaf of ideals of $\mathscr{O}_{X}$. Suppose that $\mathscr{I}$ defines a nowhere dense closed subset of $X$. Then there exists a coherent sheaf of ideals of $\mathscr{O}_{X}$, denoted $\overline{\mathscr{I}}$, such that

$$
(\overline{\mathscr{I}})_{x}=\overline{\mathscr{I}_{x}}
$$

for all $x \in X$.

\subsection{The integral closure of modules}

In this section we develop the notion of the integral closure of a module and we develop analogous properties for this object. In the contexts considered in this work, it seems most convenient to define the integral closure of a module using the valuative criterion. We follow the Gaffney's language for the integral closure of a module following [41].

Here we denote $\mathscr{O}_{n}:=\mathscr{O}_{\mathbb{C}^{n}, 0}$ and $m_{n}$ its maximal ideal.

Definition 1.2.1. Suppose $(X, x)$ is a complex analytic germ and that $M$ is a submodule of $\mathscr{O}_{X, x}^{p}$. Let $h \in \mathscr{O}_{X, x}^{p}$.

- We say that $\mathbf{h}$ is integral over $\mathbf{M}$ if $h \circ \varphi \in\left(\varphi^{*}(M)\right) \mathscr{O}_{1}$, for all analytic path $\varphi:(\mathbb{C}, 0) \rightarrow$ $(X, x)$.

- We say that $\mathbf{h}$ is strictly integral over $\mathbf{M}$ if $h \circ \varphi \in m_{1}\left(\varphi^{*}(M)\right) \mathscr{O}_{1}$, for all analytic path $\varphi:(\mathbb{C}, 0) \rightarrow(X, x)$.

The integral closure of $\mathbf{M}$ is defined as $\bar{M}:=\left\{h \in \mathscr{O}_{X, x}^{p} \mid h\right.$ is integral over $\left.M\right\}$ and the strict integral closure of $\mathbf{M}$ is defined as $M^{\dagger}:=\left\{h \in \mathscr{O}_{X, x}^{p} \mid h\right.$ is strictly integral over $\left.M\right\}$. Using the definition it is clear that $\bar{M}$ and $M^{\dagger}$ are submodules of $\mathscr{O}_{X, x}^{p}, M \subseteq \bar{M}$ and $M^{\dagger} \subseteq \bar{M}$.

Example 1.2.2. ([41] Example 1.4) Suppose $X=\mathbb{C}^{2}$ and that $M \subseteq \mathscr{O}_{2}^{2}$ is generated by $\{(x, 0),(0, y),(y, x)\}$. Then, $\bar{M}=m_{2} \mathscr{O}_{2}^{2}$. Indeed, let $\varphi:(\mathbb{C}, 0) \rightarrow\left(\mathbb{C}^{2}, 0\right)$. Then, $\left(\varphi^{*}(M)\right) \mathscr{O}_{1}$ is generated by $\left\{\left(t^{n}, 0\right),\left(0, t^{n}\right)\right\}$, where $n=\min \left\{o\left(\varphi_{1}\right), o\left(\varphi_{2}\right)\right\}$ and $o\left(\varphi_{i}\right)$ denotes the order of vanishing of $\varphi_{i}$, for $i \in\{1,2\}$. But these are the generators of $\left(\varphi^{*}\left(m_{2}\right) \mathscr{O}_{2}^{2}\right) \mathscr{O}_{1}$ as well. 
If $(X, x)$ has several components, then it is clear that $M$ induces a submodule $M_{V}$ in $\mathscr{O}_{V, x}^{p}$, $V$ any component of $(X, x)$. It is also clear from the definition that $h \in \bar{M}$ if and only if $h_{V} \in \overline{M_{V}}$ for all components $V$ of $(X, x)$, where $h_{V}$ is the element of $\mathscr{O}_{V, x}^{p}$ induced by $h$. It is clear from the definition that $\overline{\bar{M}}=\bar{M}$.

The following generalization of Nakayama's Lemma is often useful.

Proposition 1.2.3. ([41] Proposition 1.5) Suppose N, $M$ are submodules of $\mathscr{O}_{X, x}^{p}$, and $m=m_{X, x}$.

a) If $\overline{(m \bar{M}+N)}=\bar{M}$ then $\bar{N}=\bar{M}$;

b) If $M \subseteq m \bar{M}+N \subseteq \bar{M}$ then $\bar{N}=\bar{M}$.

The link between the integral closure of ideals and modules is very strong. If $M$ is a submodule of $\mathscr{O}_{X, x}^{p}$, and $[M]$ is a matrix of generators of $M$, let $J_{k}(M)$ denote the ideal generated by $k \times k$ minors of $[M]$. This is the same as the $(p-k)$-th Fitting ideal of $\frac{\mathscr{O}_{X, x}^{p}}{M}$, hence is independent of the choice of generators of $M$ (see [36] and [68]). If $h \in \mathscr{O}_{X, x}^{p}$, let $(h, M)$ denote the submodule generated by $h$ and $M$.

The following lemma, which is a generalization of Cramer's rule, is helpful in establishing the connection between $\bar{M}$ and $\overline{J_{k}(M)}$.

Lemma 1.2.4. ([41] Lemma 1.6) Suppose $h \in \mathscr{O}_{X, x}^{p}, M$ is an $\mathscr{O}_{X, x}$-submodule of $\mathscr{O}_{X, x}^{p}, J_{k+1}(h, M)=$ 0 and no element of $J_{k}(M)$ is a zero divisor on $\mathscr{O}_{X, x}$. Then

$$
J_{k}(M) \cdot h \subseteq J_{k}(h, M) \cdot M
$$

The link between $\bar{M}$ and $\overline{J_{k}(M)}$ is established by

Proposition 1.2.5. ([41] Proposition 1.7) Suppose $M$ is a submodule of $\mathscr{O}_{X, x}^{p}, X$ irreducible, $h \in \mathscr{O}_{X, x}^{p}$. Then $h \in \bar{M}$ if and only if $J_{k}(h, M) \subseteq \overline{J_{k}(M)}$, where $k$ is the largest integer such that $J_{k}(h, M) \neq 0$.

The proof of the above proposition in [41] shows that $h \in \bar{M}$ implies $J_{k}(h, M) \subseteq \overline{J_{k}(M)}$ for all $k$ with no assumptions on $X$ or the generic rank of $M$.

If $X$ is not irreducible, one obtains:

Corollary 1.2.6. ([41] Corollary 1.8) Suppose $(X, x)$ is a complex analytic germ with irreducible components $\left(V_{i}\right)$, and $M$ is a submodule of $\mathscr{O}_{X, x^{\prime}}^{p}$. Then $h \in \bar{M}$ if and only if $J_{k_{i}}\left(h, M_{i}\right) \subseteq \overline{J_{k_{i}}\left(M_{i}\right)}$, for all $i$, where $M_{i}$ is the submodule of $\mathscr{O}_{V_{i}, x}^{p}$ induced from $M$ and $k_{i}$ is the generic rank of $\left(h, M_{i}\right)$ on $V_{i}$.

In [87] Rees defined the notion of the integral closure of $M$ in $K \underset{R}{\otimes} M$, where $R$ is a noetherian domain, $K$ its field of fractions and $M$ is a finitely generated torsion-free $R$-module. 
His definition is based on the theory of discrete valuations. However, the previous proposition and the Theorem 1.2 of [87] show that $\bar{M}$ in our language is exactly the set of elements of $\mathscr{O}_{X, x}^{p}$ which are integral over $M$ in Rees's language.

Using Proposition 1.2.5 and Corollary 1.2.6, Gaffney proved the next result.

Proposition 1.2.7. ([41] Proposition 1.9) Suppose $M$ is a submodule of $\mathscr{O}_{X, x}^{p}$ and $h \in \mathscr{O}_{X, x}^{p}$. Then $h \in \bar{M}$ if and only if on each component $V$ of $(X, x)$, there exists an ideal $I$ of $\mathscr{O}_{V, x}, I \neq 0$ such that $I \cdot h \subseteq I \cdot M$ in $\mathscr{O}_{V, x}^{p}$.

In the particular case when the generic rank of $M$ is 0 or maximal, Gaffney obtained a description of the integral closure of $M$ stronger than the Proposition 1.2.7.

Proposition 1.2.8. ([41] Proposition 1.10) Suppose $M$ is a submodule of $\mathscr{O}_{X, x}^{p}$ of generic rank $p$ or 0 on each component of $X$, and let $h \in \mathscr{O}_{X, x}^{p}$. Then $h \in \bar{M}$ if and only if there exists a faithful submodule I of $\mathscr{O}_{X, x}$ such that $I \cdot h \subseteq I \cdot M$.

The next proposition contains the growth condition for integral closure in the module case. The version we work here was suggested by Looijenga. In what follows, we let $\Gamma(E)$ denote sections of a vector bundle.

Proposition 1.2.9. ([41] Proposition 1.11) Suppose $h \in \mathscr{O}_{X, x}^{p}$ and that $M$ is a submodule of $\mathscr{O}_{X, x}^{p}$. Then $h \in \bar{M}$ if and only if for each choice of generators $\left\{s_{i}\right\}$ of $M$ there exists a neighborhood $U$ of $x$ such that for all $\varphi \in \Gamma\left(\operatorname{Hom}\left(\mathbb{C}^{p}, \mathbb{C}\right)\right)$,

$$
\|\varphi(z) \cdot h(z)\| \leq C \sup _{i}\left\|\varphi(z) \cdot s_{i}(z)\right\|
$$

for all $z \in U$.

We consider the "sheafification"of the construction of the integral closure of modules. The connection between $M$ and $J_{k}(M)$ allows us to show that the integral closure gives rise to a coherent sheaf. The proof uses a description of $\bar{M}$ in terms of blowing up.

Proposition 1.2.10. ([41] Proposition 1.12) Suppose $\mathscr{M}$ is a coherent sheaf of submodules of $\mathscr{O}_{X}^{p}$. Then, there exists a unique coherent sheaf $\overline{\mathscr{M}}$ on $X$ such that for each $x \in X,(\overline{\mathscr{M}})_{x}=\overline{\mathscr{M}}_{x}$.

There is another way to make a connection between the integral closure of modules and ideals.

Let us define the ideal sheaf $\rho(M)$ on $X \times \mathbb{P}^{p-1}$ associated to a submodule sheaf $M$ of $\mathscr{O}_{X}^{p}($ see $[48])$ : Given $h=\left(h_{1}, \ldots, h_{p}\right) \in \mathscr{O}_{X}^{p}$ and $\left(x,\left[t_{1}, \ldots, t_{p}\right]\right) \in X \times \mathbb{P}^{p-1}$, with $t_{i} \neq 0$, we define $\rho(h)$ as the germ of the analytic map given by $\sum_{j=1}^{p} h_{j}(z) \frac{T_{j}}{T_{i}}$ which is well-defined on a Zariski open subset of $X \times \mathbb{P}^{p-1}$ that contains the point $\left(x,\left[t_{1}, \ldots, t_{p}\right]\right)$. We define $\rho(M)$ as the ideal generated by $\{\rho(h) \mid h \in M\}$. 
The next result gives a relation between the integral closure of modules and ideals.

Proposition 1.2.11. ([48] Proposition 3.4) Let $h \in \mathscr{O}_{X, x}^{p}$. Then $h \in \bar{M}$ at $x$ if and only if $\rho(h) \in$ $\overline{\rho(M)}$ at all point $\left(x,\left[t_{1}, \ldots, t_{p}\right]\right) \in V(\rho(M))$.

\section{The Multiplicity of a Pair of Modules}

We end this section defining the multiplicity of a pair of modules following the lines of [42].

The multiplicity of an ideal or module or pair of modules is one of the most important invariants we can associate to an $m$-primary module. It is intimately connected with integral closure.

Let $(X, x)$ be a germ of an analytic space and assume that $(X, x)$ has dimension $d$. Let $M \subseteq N$ be sheaves of $\mathscr{O}_{X}$-submodules of $\mathscr{O}_{X}^{p}$, where $M$ has finite colength in $N$. Denote $\mathscr{M}$ the associated ideal sheaf $\rho(M)$. Let $\mathscr{R}(M)$ and $\mathscr{R}(N)$ the Rees algebra of $M$ and $N$, respectively.

Then, we have canonical maps which give us the following diagram:

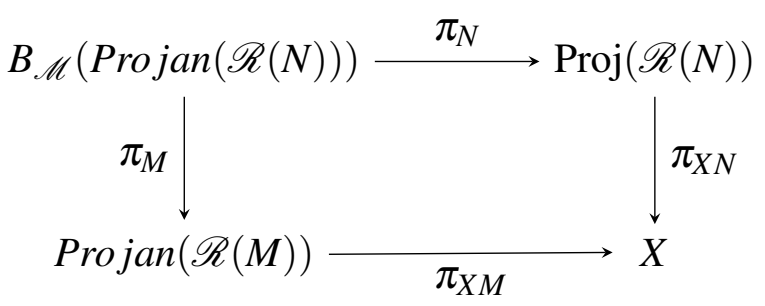

On the blow up $B_{\mathscr{M}}(\operatorname{Projan}(\mathscr{R}(N)))$ we have two tautological bundles. One is the pullback of the bundle on Projan $(\mathscr{R}(N))$. The other comes from Projan $(\mathscr{R}(M))$. Denote the corresponding Chern classes by $c_{M}$ and $c_{N}$, and denote the exceptional divisor by $D_{M, N}$. Suppose the generic rank of $N$ (and hence $M$ ) is $g$.

Then, the multiplicity of a pair of modules $(M, N)$ at $x$ is:

$$
e(M, N):=\sum_{j=0}^{d+g-2} \int D_{M, N} \cdot c_{M}^{d+g-2-j} \cdot c_{N}^{j} .
$$

Kleiman and Thorup show that this multiplicity is well defined at $x \in X$ as long as $\bar{M}=\bar{N}$ on a deleted neighborhood of $x$. This condition implies that $D_{M, N}$ lies in the fiber over $x$, hence is compact. It also possible to conclude that, when $N=\mathscr{O}_{X, x}^{p}$, and $M$ has finite colength in $\mathscr{O}_{X, x}^{p}$ then $e\left(M, \mathscr{O}_{X, x}^{p}\right)$ is the Buchsbaum-Rim multiplicity $e(M)$ defined in [19]. There is a fundamental result due to Kleiman and Thorup, the principle of additivity [62], which states that given a sequence of $\mathscr{O}_{X, x}$-modules $M \subseteq N \subseteq P$ such that the multiplicity of the pairs is well defined, then

$$
e(M, P)=e(M, N)+e(N, P)
$$


Also if $\bar{M}=\bar{N}$ at $x$ then $e(M, N)=0$, and the converse also holds if $X$ is equidimensional. These results will be used in this work.

\subsection{Whitney equisingularity and the integral closure of modules}

In this section, we follow the Gaffney's approach about Whitney equisingularity and integral closure of modules presented in [42].

The way how Gaffney got algebraic conditions that characterizes the Whitney equisingularity using the integral closure of modules have inspired us to develop some algebraic tools in this work that will be useful in order to describe the bi-Lipchitz Equisingularity.

We start with some notation to describe a family of analytic sets.

Setup 1.3.1. Let $(X, 0) \subseteq\left(\mathbb{C}^{n+k}, 0\right)$ be the germ of the analytic space defined by an analytic map $F: \mathbb{C}^{n} \times \mathbb{C}^{k} \rightarrow \mathbb{C}^{p}$, where $X$ is a sufficient small representative such that $Y=\mathbb{C}^{k}=0 \times \mathbb{C}^{k} \subseteq X$ is the singular set of $X$. Let $F_{1}, \ldots, F_{p}: \mathbb{C}^{n} \times \mathbb{C}^{k} \rightarrow \mathbb{C}$ be the coordinates functions of $F$, for each $y \in Y$ let $f_{y}: \mathbb{C}^{n} \rightarrow \mathbb{C}^{p}$ given by $f_{y}(z):=F(z, y)$ and let $X_{y}:=f_{y}^{-1}(0)$. Assume that $X_{y}$ has an isolated singularity in 0 , for all $y \in Y$ and $n \geq p$. Let $z_{1}, \ldots, z_{n}, y_{1}, \ldots, y_{k}$ be the coordinates on $\mathbb{C}^{n+k}$, let $m_{Y}$ be the ideal of $\mathscr{O}_{X}$ generated by $\left\{z_{1}, \ldots, z_{n}\right\}$, let $J M(X)$ be the jacobian module of $X$, let $J M(X)_{Y}$ be the module generated by $\left\{\frac{\partial F}{\partial y_{1}}, \ldots, \frac{\partial F}{\partial y_{k}}\right\}$ and let $J M_{z}(X)$ be the module generated by $\left\{\frac{\partial F}{\partial z_{1}}, \ldots, \frac{\partial F}{\partial z_{n}}\right\}$. In this setup we assume that $F$ defines $X$ with reduced structure and $X$ is equidimensional. Usually $Y$ is called the parameter space of the family $X$.

Given a family as above, we say the family is holomorphically trivial if there exists a holomorphic family of origin preserving bi-holomorphic germs $r_{y}$ such that $r_{y}\left(X_{0}\right)=X_{y}$. If the map-germs are only homeomorphisms, we say the family is $C^{0}$ trivial.

Example 1.3.2. ([42] Example 1.1) Let $X \subseteq \mathbb{C}^{3}$ be the family of four moving lines with equation $F(x, y, z)=x z(z+x)(z-(1+y) x)=0$. Here $y$ is the parameter, the $x$ and $z$ axis are fixed as is the line $z+x=0$, while the line $z=(1+y) x$ moves with $y$.

For each $y \in \mathbb{C}$ let $f_{y}:\left(\mathbb{C}^{2}, 0\right) \rightarrow \mathbb{C}$ given by $f_{y}(z)=F(x, y, z)$.

It is easy to see that if $\theta: \mathbb{C}^{2} \rightarrow \mathbb{C}^{2}$ is a $\mathbb{C}$-linear map which preserves the lines $x=0$, $z=0$ and $z=-x$ on $\mathbb{C}^{2}$ then $\theta$ is a multiple of the identity on $\mathbb{C}^{2}$.

Suppose by contradiction that $X$ is a holomorphically trivial family. So we have a family $r_{y}:\left(\mathbb{C}^{2}, 0\right) \rightarrow\left(\mathbb{C}^{2}, 0\right), y \in \mathbb{C}$, of bi-holomorphic germs such that $r_{y}\left(X_{0}\right)=X_{y}$, for all $y \in \mathbb{C}$. Take $y \in \mathbb{C}, y \neq 0$. Since $r_{y}\left(X_{0}\right)=X_{y}$ then the differential Dr $r_{y}(0): \mathbb{C}^{2} \rightarrow \mathbb{C}^{2}$ takes the tangent lines of $X_{0}$ into the tangent lines of $X_{y}$, i.e:

$$
\{z=-x\} \subseteq X_{0} \text { maps to }\{z=-x\} \subseteq X_{y} \text { through } \operatorname{Dr}_{y}(0)
$$




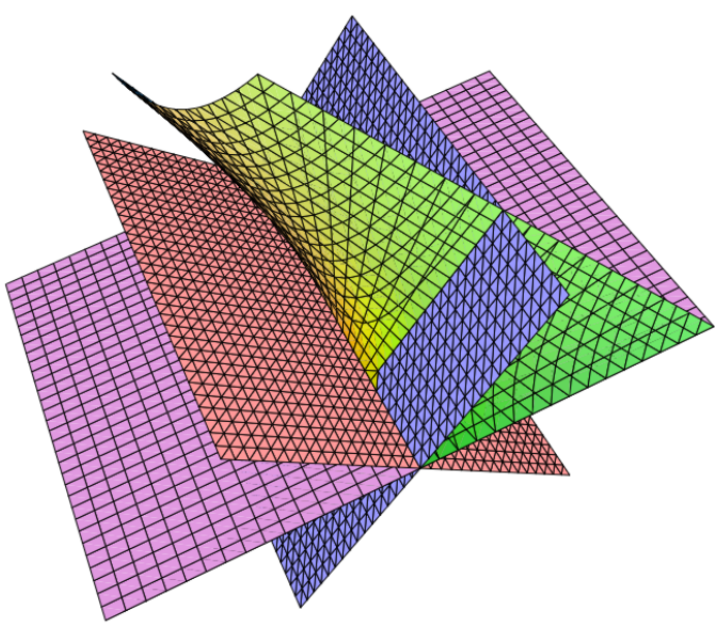

Figure 1 - Four moving lines

$$
\begin{aligned}
& \{z=0\} \subseteq X_{0} \text { maps to }\{z=0\} \subseteq X_{y} \text { through } \operatorname{Dr}_{y}(0) \\
& \{x=0\} \subseteq X_{0} \text { maps to }\{x=0\} \subseteq X_{y} \text { through } \operatorname{Dr}_{y}(0) \\
& \{z=x\} \subseteq X_{0} \text { maps to }\{z=x\} \subseteq X_{y} \text { through } \operatorname{Dr}_{y}(0) .
\end{aligned}
$$

In particular $\operatorname{Dr}_{y}(0)$ is a $\mathbb{C}$-linear map which preserves the lines $x=0, z=0$ and $z=-x$ on $\mathbb{C}^{2}$. Thus, Dry $(0)$ is a multiple of the identity, i.e, there exists $a \in \mathbb{C}$ such that Dry $(0)=a . I d_{\mathbb{C}^{2}}$. Taking $x \neq 0$ sufficiently nearby 0 , once we apply $\operatorname{Dr}_{y}(0)$ at the point $(x, x)$, we conclude that $a=1$ and $a=1+y$, which implies that $y=0$, contradiction.

Therefore, the family $X$ is not holomorphically trivial. However it should be equisingular for any reasonable definition of equisingularity.

This example shows that we need a notion of equisingularity that is less restrictive than holomorphic equivalence.

The Whitney conditions imply $C^{0}$-triviality but also imply the family is well-behaved at the infinitesimal level.

Here we denote $\mathbb{K}$ as the field $\mathbb{R}$ or $\mathbb{C}$.

Definition 1.3.3. (Whitney's conditions) Suppose $X$ is a subset of $\mathbb{K}^{n}, X_{0}$ is the set of smooth points on $X$ and that $Y$ is a smooth subset of $X$.

- The pair $\left(X_{0}, Y\right)$ satisfies Whitney's condition $\boldsymbol{A}$ at $y \in Y$ iffor all sequences $\left(x_{i}\right)$ of points of $X_{0}$ such that

$$
\begin{gathered}
x_{i} \rightarrow y \\
T_{x_{i}} X \rightarrow T
\end{gathered}
$$

implies $T_{y} Y \subseteq T$; 
- The pair $\left(X_{0}, Y\right)$ satisfies Whitney's condition $\boldsymbol{B}$ at $y \in Y$ iffor all sequences $\left(x_{i}\right)$ of points of $X_{0}$ and $\left(y_{i}\right)$ of points of $Y$ such that

$$
\begin{gathered}
x_{i} \rightarrow y \\
y_{i} \rightarrow y \\
T_{x_{i}} X \rightarrow T \\
\sec \left(x_{i}, y_{i}\right) \rightarrow L
\end{gathered}
$$

implies $L \subseteq T$.

John Mather first pointed out that Whitney's condition B implies Whitney's condition A in the notes of his lectures at Harvard in 1970 [69].

Example 1.3.4. Let us go back to the Example 1.3.2, and to prove that the family of four moving lines satisfies the Whitney's conditions. As pointed out by Mather, we only have to check the condition $B$.

Let $Y$ be a smooth subspace of $X, u \in Y,\left(x_{i}\right)_{i \in \mathbb{N}}$ a sequence of points of $X_{0}$ and $\left(y_{i}\right)_{i \in \mathbb{N}} a$ sequence of points of $Y$ such that

$$
\begin{gathered}
x_{i} \rightarrow u \\
y_{i} \rightarrow u \\
T_{x_{i}} X \rightarrow T \\
\sec \left(x_{i}, y_{i}\right) \rightarrow L .
\end{gathered}
$$

Let $M, N$ and $P$ the planes $x=0, z=0$ and $z=-x$, respectively, and let $R$ the surface $z=(1+y) x$. So $M \cap N \cap P \cap R$ is the $y$-axis and $X=M \cup N \cup P \cup R$. Consider

$$
\begin{aligned}
\tilde{M} & :=\left\{i \in \mathbb{N} \mid x_{i} \in M\right\} \\
\tilde{N} & :=\left\{i \in \mathbb{N} \mid x_{i} \in N\right\} \\
\tilde{P} & :=\left\{i \in \mathbb{N} \mid x_{i} \in P\right\} \\
\tilde{R} & :=\left\{i \in \mathbb{N} \mid x_{i} \in R\right\} .
\end{aligned}
$$

Clearly $\mathbb{N}=\tilde{M} \cup \tilde{N} \cup \tilde{P} \cup \tilde{R}$ and one of these sets has to be not bounded.

(i) Suppose that $\tilde{M}$ is not bounded. So we have a subsequence $\left(T_{x_{i}} X\right)_{i \in \tilde{M}} \rightarrow T$, hence $T=M$. We also have that $u=\lim _{i \rightarrow \infty} x_{i}=\lim _{i \in \tilde{M}} x_{i} \in \bar{M}=M$ which implies that $T_{u} M=M$. Since $Y$ is a smooth subset of $X$ and $u \in M$ there exists an open subset $U$ of $Y$ such that $u \in U$ and $U$ is a submanifold of $M$ (this occurs because $M, N, P$ and $R$ intersects transversely pairwise). Since $\left(x_{i}\right),\left(y_{i}\right) \rightarrow u$ then $x_{i}, y_{i} \in U \subseteq M, \forall i \in \mathbb{N}$ sufficiently large. Therefore, the set $\hat{M}:=\left\{i \in \mathbb{N} \mid x_{i}, y_{i} \in M\right\}$ is not bounded. Since $M$ is a plane then $\sec \left(x_{i}, y_{i}\right) \subseteq M, \forall i \in \hat{M}$ and since this subsequence also converges to $L$ then $L \subseteq M=T$. 
The same argument can be applied when $\tilde{N}$ or $\tilde{P}$ once these subsets of $X$ are planes as well.

(ii) Suppose that $\tilde{R}$ is not bounded. Analogously to the previous case, we have that $u \in R$, there exists an open subset $U$ of $Y$ such that $u \in U$ such that $U$ is a submanifold of $R$ and the set $\hat{R}:=\left\{i \in \mathbb{N} \mid x_{i}, y_{i} \in R\right\}$. Since $R$ and $U$ is a submanifold of $R$ then the pair $(R, U)$ satisfies the Whitney's conditions. Since

$$
\begin{gathered}
\left(x_{i}\right)_{i \in \hat{R}} \rightarrow u \\
\left(y_{i}\right)_{i \in \hat{R}} \rightarrow u \\
\left(T_{x_{i}} R\right)_{i \in \hat{R}} \rightarrow T \\
\left(\sec \left(x_{i}, y_{i}\right)\right)_{i \in \hat{R}} \rightarrow L .
\end{gathered}
$$

then $L \subseteq T$.

Therefore, the family of four moving lines satisfies the Whitney's conditions.

Example 1.3.5. ([42] Example 1.4) Consider $X \subseteq \mathbb{R}^{3}$ the family defined by $F(x, y, z)=z^{3}+x^{2}-$ $y^{2} z^{2}=0$. The members of the family $X_{y}$ consist of node singularities where the loop is pulled smaller and smaller as $y$ tends to zero becoming a cusp at $y=0$ (see the picture below). The singular locus is the y-axis. Whitney A holds because every limiting tangent plane contains the $y$-axis. But Whitney $B$ fails. Notice that the parabola $z=y^{2}$ is in the surface, and letting $x_{i}=\left(0, t_{i}, t_{i}^{2}\right)$ and $y_{i}=\left(0, t_{i}, 0\right),\left(t_{i}\right)$ any sequence tending to 0 , we see that the limiting secant line is the z-axis, while the limiting tangent plane along this curve is the xy-plane.

We see that the dimension of the limiting tangent planes at the origin is 1, while it is zero elsewhere on the y-axis. This kind of drastic change at the infinitesimal level is prevented by the Whitney conditions.

Reading: There are many places that we can read about the Whitney conditions. An important reference is the Chapter III of [98], which is more in the spirit of the way that Gaffney develops the subject. Surely one can see the works of Whitney $[109,110]$ as well.

\section{Verdier's Condition W (or (w)-regularity)}

The next condition, while is equivalent to the Whitney conditions in the complex analytic case (proved by Teissier in [98]), is easier to work using algebra.

The $(w)$-regularity says that the distance between the tangent space to $X$ at a point $x_{i}$ of $X_{0}$ and the tangent space to $Y$ at $y$ goes to zero as fast as the distance between $x_{i}$ and $Y$. We first need to define what we mean by the distance between two linear spaces.

Suppose $A, B$ are linear subspaces of $\mathbb{K}^{N}$. We define the distance from $A$ to $B$ as

$$
\operatorname{dist}(A, B)=\sup \left\{\frac{\|\langle u, v\rangle\|}{\|u\|\|v\|} \mid u \in B^{\perp}-\{0\} \text { and } v \in A-\{0\}\right\} \text {. }
$$




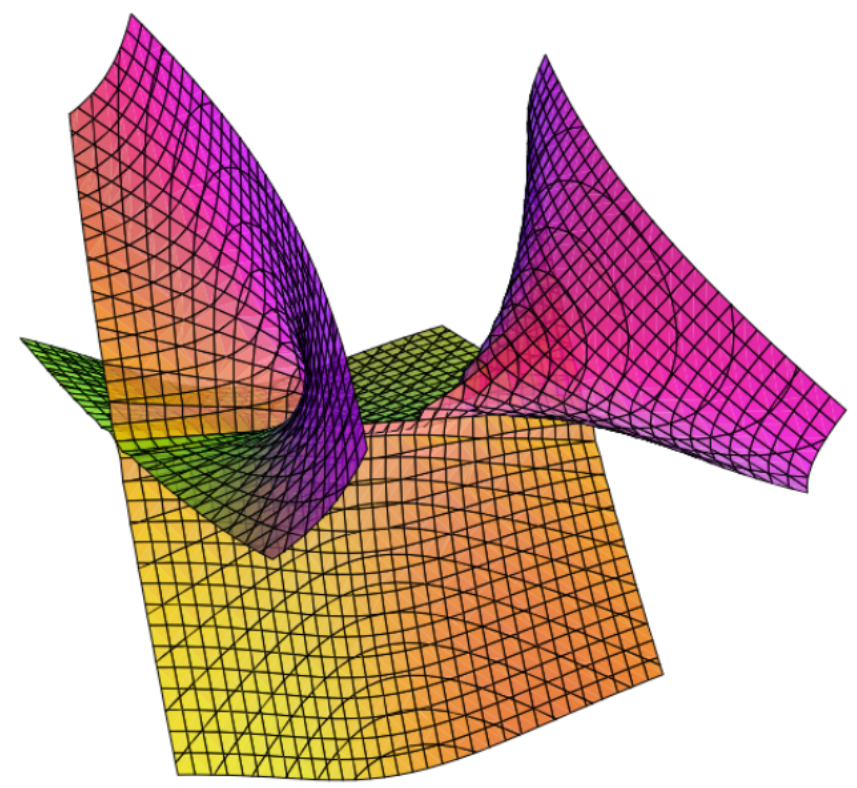

Figure $2-X: y^{2} z^{2}=z^{3}+x^{2}$

In the applications, $B$ is the "big space"and $A$ is the "small" space. The inner product is Hermitian inner product when we work over $\mathbb{C}$.

Example 1.3.6. ([42] Example 1.5) Work in $\mathbb{R}^{3}$. Let $A=x$-axis, $B$ a plane with unit normal $u_{0}$, then the distance from $A$ to $B$ is $\cos \theta$, where $\theta$ is the small angle between $u_{0}$ and the $x$-axis, in the plane they determine. So when the distance is $0, B$ contains the $x$-axis.

We recall Verdier's condition $W$, also called $(w)$-regularity.

Definition 1.3.7. Suppose $Y \subseteq \bar{X}$, where $X, Y$ are strata in a stratification of an analytic space. Then the pair $(X, Y)$ satisfies the Verdier's condition $W$ at $0 \in Y$ if there exists a positive real number $C$ such that $\operatorname{dist}\left(T Y_{0}, T X_{x}\right) \leq C \operatorname{dist}(x, Y)$ for all $x$ close to $Y$.

The next theorem was proved first by Teissier in the case of a family of analytic hypersurfaces, and generalized by Gaffney, for any codimension.

Theorem 1.3.8. ([41] Theorem 2.5) With the setup 1.3.1, the Verdier's condition Wholds for $(X-Y, Y)$ at $(0,0)$ if and only if $(X-Y, Y)$ satisfies the Whitney's conditions at $(0,0)$ if and only if

$$
J M(X)_{Y} \subseteq \overline{m_{Y} J M_{z}(X)}
$$

\subsection{The Lipschitz saturation and the double of an ideal}

Following the approach of Pham-Teissier in [85], let $A$ be a commutative local ring over $\mathbb{C}$, and $\bar{A}$ its normalization (we can assume that $A$ is the local ring of an anatyc space $X$ at the 
origin in $\mathbb{C}^{n}$ ). Let $I$ be the kernel of the inclusion

$$
\bar{A} \underset{\mathbb{C}}{\otimes} \bar{A} \rightarrow \bar{A} \underset{A}{\otimes} \bar{A}
$$

In this construction, the tensor product is the analytic tensor product which has the right universal property for the category of analytic algebras, and which gives the analytic algebra for the analytic fiber product.

Pham and Teissier then defined the Lipschitz saturation of $A$, denoted $\tilde{A}$, to consist of all elements $h \in \bar{A}$ such that $h \otimes 1-1 \otimes h \in \bar{A} \otimes \bar{C}$ is in the integral closure of $I$ (for related results see [66]). The connection between this notion and that of Lipschitz functions is as follows. If we pick generators $\left(z_{1}, \ldots, z_{n}\right)$ of the maximal ideal of the local ring $A$, then $z_{i} \otimes 1-1 \otimes z_{i} \in \bar{A} \underset{\mathbb{C}}{\otimes} \bar{A}$ give a set of generators of $I$. Choosing $z_{i}$ so that they are the restriction of coordinates on the ambient space, using the supremum criterion given by Lejeune-Jalabert and Teissier in [64], we see the integral closure condition is equivalent to

$$
\left|h\left(z_{1}, \ldots, z_{n}\right)-h\left(z_{1}^{\prime}, \ldots, z_{n}^{\prime}\right)\right| \leq C \sup _{i}\left\{\left|z_{i}-z_{i}^{\prime}\right|\right\}
$$

holding on some neighborhood $U$ of $(0,0)$ on $X \times X$. This last inequality is what is meant by the meromorphic function $h$ being Lipschitz at the origin on $X$. Note that the integral closure condition is equivalent to the inequality holding on a neighborhood $U$ for some $C$ for any set of generators of the maximal ideal of the local ring. The constant $C$ and the neighborhood $U$ will depend on the choice.

If $(X, x)$ is normal then passing to the Lipschitz saturation does not add any functions.

Definition 1.4.1. Let I be an ideal of $\mathscr{O}_{X, x}, S B_{I}(X)$ the Lipschitz saturation of the blow-up, which is the space whose analytic sheaf of rings $\mathscr{O}_{S B_{I}(X)}$ is the Lipschitz saturation of the analytic sheaf of rings $\mathscr{O}_{B_{I}(X)}$, i.e,

$$
\mathscr{O}_{S B_{I}(X)}=\widetilde{\mathscr{O}}_{B_{I}(X)}
$$

Let $\pi_{S}: S B_{I}(X) \rightarrow X$ be the projection map. The Lipschitz saturation of the ideal I is denoted $I_{S}$, and is the ideal $I_{S}:=\left\{h \in \mathscr{O}_{X, x} \mid \pi_{S}^{*}(h) \in \pi_{S}^{*}(I)\right\}$.

Since the normalization of a local ring $A$ contains the Lipschitz Saturation of $A$ then $I \subseteq I_{S} \subseteq \bar{I}$. In particular, if $I$ is integrally closed then $I_{S}=\bar{I}$.

Here is a viewpoint on the Lipschitz saturation of an ideal $I$, which will be useful later. Given an ideal $I$, and an element $h$ that we want to check for inclusion in $I_{S}$, we can consider $\left(B_{I}(X), \pi\right), \pi^{*}(I)$ and $h \circ \pi$. Since $\pi^{*}(I)$ is locally principal, working at a point $z$ on the exceptional divisor $E$, we have a local generator $f \circ \pi$ of $\pi^{*}(I)$. Consider the quotient $(h / f) \circ \pi$. Then $h \in I_{S}$ if and only if at the generic point of any component of $E,(h / f) \circ \pi$ is Lipschitz with respect to a system of local coordinates. If this holds we say $h \circ \pi \in\left(\pi^{*}(I)\right)_{S}$. 
We can also work on the normalized blow-up $\left(N B_{I}(X), \pi_{N}\right)$. Then we say $h \circ \pi_{N} \in$ $\left(\pi_{N}^{*}(I)\right)_{S}$ if $(h / f) \circ \pi_{N}$ satisfies a Lipschitz condition at the generic point of each component of the exceptional divisor of $\left(N B_{I}(X), \pi_{N}\right)$ with respect to the pullback to $\left(N B_{I}(X), \pi_{N}\right)$ of a system of local coordinates $B_{I}(X)$ at the corresponding points of $B_{I}(X)$. As usual, the inequalities at the level of $N B_{I}(X)$ can be pushed down and are equivalent to inequalities on a suitable collection of open sets on $X$.

This definition can be given in an equivalent statement using the theory of integral closure of modules. Since Lipschitz conditions depend on controlling functions at two different points as the points come together, we should look for a sheaf defined on $X \times X$. We describe a way of moving from a sheaf of ideals on $X$ to a sheaf on $X \times X$.

Let $\pi_{1}, \pi_{2}: X \times X \rightarrow X$ be the projections to the $i$-th factor, $i \in\{1,2\}$, and let $h \in \mathscr{O}_{X, x}$. Define $h_{D} \in \mathscr{O}_{X \times X,(x, x)}^{2}$ as $\left(h \circ \pi_{1}, h \circ \pi_{2}\right)$, called the double of $\mathbf{h}$. We define the double of the ideal $I$, denoted $I_{D}$, as the submodule of $\mathscr{O}_{X \times X,(x, x)}^{2}$ generated by $h_{D}$, where $h$ is an element of $I$.

If $I$ is an ideal sheaf on a space $X$ then intuitively, $h \in \bar{I}$ if $h$ tends to zero as fast as the elements of $I$ do as you approach a zero of $I$. If $h_{D}$ is in $\overline{I_{D}}$ then the element defined by $(1,-1) \cdot\left(h \circ \pi_{1}, h \circ \pi_{2}\right)=h \circ \pi_{1}-h \circ \pi_{2}$ should be in the integral closure of the ideal generated by applying $(1,-1)$ to the generators of $I_{D}$, namely the ideal generated by $g \circ \pi_{1}-g \circ \pi_{2}, g$ any element of $I$. This implies the difference of $h$ at two points goes to zero as fast as the difference of elements of $I$ at the two points go to zero as the points approach each other. It is reasonable that elements in $I_{S}$ should have this property. In fact, as we can see in [40], the following result gives a nice link between Lipschitz saturation and integral closure of modules.

Theorem 1.4.2. ([40] Theorem 2.3) Suppose $(X, x)$ is a complex analytic set germ, $I \subseteq \mathscr{O}_{X, x}$ is an ideal and $h \in \mathscr{O}_{X, x}$. Then $h \in I_{S}$ if and only if $h_{D} \in \overline{I_{D}}$.

Corollary 1.4.3. ([39] Corollaries 3.4 and 3.5) Let $I \subseteq J \subseteq \bar{I}$ be ideals of $\mathscr{O}_{X, x}$, with $X$ equidimensional. Suppose that $I_{D}$ has finite colenght in $J_{D}$ and $J_{D}$ has finite colenght in $(\bar{I})_{D}$. Then

$$
e\left(I_{D},(\bar{I})_{D}\right)=e\left(J_{D},(\bar{I})_{D}\right) \text { if and only if } \overline{I_{D}}=\overline{J_{D}}
$$

if and only if

$$
I_{S}=J_{S}
$$

Here is an example showing the difference between the integral closure of the jacobian ideal and its saturation, in particular, the difference between the integral closure and saturation. 
Example 1.4.4. ([39]) Consider $F(x, y)=x^{2}+y^{p}, p \geq 3$ odd. Denote by $X$ the plane curve defined by $F$. Then $X$ has a normalization given by $\phi(t)=\left(t^{p}, t^{2}\right)$. The elements in the integral closure of the jacobian ideal are just those ring elements $h$ such that $h \circ \phi \in \phi^{*}(J(F))=\left(t^{p}\right)$. Now, $y^{q} \circ \phi=t^{2 q}$, so $y^{q} \in \overline{J(F)}$, for $q>\frac{p}{2}$. Denote a matrix of generators for $J(F)_{D}$ by $\left[J(F)_{D}\right]$. Consider the curve mapping into $X \times X$ given by $\Phi(t)=\left(t^{p}, t^{2}, t^{p}, c t^{2}\right)$, where $c$ is a $p^{\text {th }}$ root of unity different from 1 . Now consider the ideal generated by the entries of the vector

$$
(1,-1)\left[J(F)_{D}\right] \circ \Phi(t)
$$

This ideal is generated by $\left(y^{p-1}-y^{p-1},\left(y-y^{\prime}\right)\left(x, x^{\prime}, y^{p-1}, y^{\prime p-1}\right)\right) \circ \Phi(t)=\left(t^{p+2}\right)$. Meanwhile the order in $t$ of $(1,-1)\left(y^{q}, y^{q}\right) \circ \Phi(t)$ is $2 q$. If $p<2 q<p+2$, i.e, $q=\frac{p+1}{2}$, then $\left(y^{q}, y^{\prime q}\right)$ cannot be in $\overline{J(F)_{D}}$, hence $y^{q} \notin J(F)_{S}$, but $y^{q} \in \overline{J(F)}$.

Using the Lipschitz saturation of ideals (and doubles), in [39] we have set the infinitesimal Lipschitz conditions for hypersurfaces.

Setup: Let $X^{n+k}, 0 \subseteq \mathbb{C}^{n+1+k}, 0$ be a hypersurface, containing a smooth subset $Y$ embedded in $\mathbb{C}^{n+1+k}$ as $0 \times \mathbb{C}^{k}$, with $p_{Y}$ the projection to $Y$. Assume $Y=S(X)$, the singular set of $X$. Suppose $F$ is the defining equation of $X,(z, y)$ coordinates on $\mathbb{C}^{n+1+k}$. Denote by $f_{y}(z)=F(z, y)$ the family of functions defined by $F$ and $X_{y}:=f_{y}^{-1}(0)$. Assume that $f_{y}$ has an isolated singularity at the origin. Let $m_{Y}$ denote the ideal defining $Y$, and $J(F)_{Y}$, the ideal generated by the partial derivatives with respect to the $y$ coordinates, $J_{z}(F)$, those with respect to the $z$ coordinates. Here we work with the double relative to $Y$, which means that we work with the projections $\pi_{1}$ and $\pi_{2}$ defined on the fibered product $X \underset{Y}{\times} X$.

Definition 1.4.5. We say the pair $(X, Y)$ satisfies the infinitesimal Lipschitz condition $\mathbf{m}_{\mathbf{Y}}$ $\left(\mathbf{i} \mathbf{L}_{\mathbf{Y}}\right)$ at the origin if either of the two equivalent conditions hold:

$$
\begin{aligned}
& \text { 1. } J(F)_{Y} \subseteq\left(m_{Y} J_{Z}(F)\right)_{S} ; \\
& \text { 2. }\left(J(F)_{Y}\right)_{D} \subseteq \overline{\left(m_{Y} J_{Z}(F)\right)_{D}} .
\end{aligned}
$$

An analogous condition for $i L_{m_{Y}}$ is $J(F)_{Y} \subseteq \overline{m_{Y} J_{z}(F)}$. This is the equivalent to the Verdier's condition $W$ or the Whitney conditions. Next we give the definition of $i L_{A}$.

Definition 1.4.6. We say the pair $(X, Y)$ satisfies the infinitesimal Lipschiz condition $\boldsymbol{A}\left(\mathbf{i L}_{\mathbf{A}}\right)$ at the origin if either of the two equivalent conditions hold:

1. $J(F)_{Y} \subseteq\left(J_{z}(F)\right)_{S}$

2. $\left(J(F)_{Y}\right)_{D} \subseteq \overline{\left(J_{Z}(F)\right)_{D}}$.

The analogous condition is $J(F)_{Y} \subseteq \overline{J_{z}(F)}$. If one works on the ambient space, then this is equivalent to the $A_{F}$ condition. 
Since there are different ways in which the total space $X^{n+k}$ can be made into a family of spaces, it is natural to ask if the conditions we have defined depend on the projection to $Y$ which defines the family. The following result says the $i L_{m_{Y}}$ does not depend on the projection to $Y$.

Proposition 1.4.7. ([39] Proposition 3.8) In the above setup the following conditions are equivalent:

(a) $\left(J(F)_{Y}\right)_{D} \subseteq \overline{\left(m_{Y} J_{Z}(F)\right)_{D}}$;

(b) $\left(J(F)_{Y}\right)_{D} \subseteq \overline{\left(m_{Y} J(F)\right)_{D}}$.

While a similar result for $i L_{A}$ does not make sense, if we ask that $\left(J(F)_{Y}\right)_{D} \subseteq\left(J_{Z}(F)\right)_{D}^{\dagger}$ then an analogous result holds.

In Proposition 4.1 of [39] is proved that the cosupport of $\left(m_{Y} J_{z}(F)\right)_{D}$ and $\left(J_{z}(F)\right)_{D}$ on $X \underset{Y}{\times} X$ are equal, and consist of

$$
\Delta(X) \cup(\underset{Y}{X} \underset{Y}{\times}) \cup(\underset{Y}{0} \times)
$$

This set is where makes sense to ask about the infinitesimal Lipschitz conditions. The next result says us that both $i L$ conditions hold on the above set off $(0,0) \times Y$.

Proposition 1.4.8. ([39] Proposition 4.2) $i L_{A}$ and $i L_{m_{Y}}$ hold at all points of $\Delta(X)-((0,0) \times Y)$,

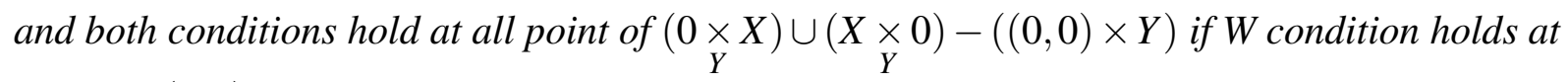
all point $(0, y), y \in Y$.

In the chapter 3 we generalize this results for an arbitrary codimension of $X$.

Finally, in $(0,0) \times Y$, Gaffney proved that the $i L_{A}$ condition holds generically.

Theorem 1.4.9. ([39] 4.3) In the setup of this section, there exists a Zariski open subset $U$ of $Y$ such that $i L_{A}$ holds for the pair $(X-Y, U \cap Y)$ along $Y$.

\subsection{Determinantal Varieties}

We first recall the definition of determinantal varieties. Let $\Sigma_{t} \subseteq \operatorname{Hom}\left(\mathbb{C}^{n}, \mathbb{C}^{p}\right)$ be the subset consisting of the maps that have rank less than $t$, with $1 \leq t \leq \min (n, p)$. It is possible to show that $\Sigma_{t}$ is an irreducible singular algebraic variety of codimension $(n-t+1)(p-t+1)$ (see [18]). Moreover the singular set of $\Sigma_{t}$ is exactly $\Sigma_{t-1}$. The set $\Sigma_{t}$ is called a generic determinantal variety of size $(n, p)$ obtained from $t \times t$ minors.

The representation of the variety $\Sigma_{t}$ as the union $\Sigma_{i} \backslash \Sigma_{i-1}, i=1, \ldots, t$ is a stratification of $\Sigma_{t}$, which is locally holomorphically trivial, and it is called the rank stratification of $\Sigma_{t}$. 
Definition 1.5.1. Let $U \subseteq \mathbb{C}^{r}$ be an open domain, $F=\left(m_{i j}(x)\right)$ be an $n \times p$ matrix whose entries are complex analytic functions on $U, 0 \in U$ and $f$ the function defined by the $t \times t$ minors of $M$. We say that $X=V(f)$ is a determinantal variety if it has codimension $(n-t+1)(p-t+1)$.

Currently, determinantal varieties have been an important object of study in Singularity Theory. For example, we can refer to the works of Damon [29], Frühbis-Krüger [37, 38], Gaffney [39, 47], Grulha [47], Nuño-Ballesteros [2, 76], Oréfice-Okamoto [2, 76], Pereira [83, 89], Pike [29], Ruas [47, 89], Tomazella [2, 76], Zhang [114] and others.

In the case where $X$ is a codimension two determinantal variety, we can use the HilbertBurch theorem to obtain a good description of $X$ and its deformations in terms of its presentation matrix. In fact, if $X$ is a codimension two Cohen- Macaulay variety, then $X$ can be defined by the maximal minors of a $n \times(n+1)$ matrix. Moreover, any perturbation of a $n \times(n+1)$ matrix gives rise to a deformation of $X$ and any deformation of $X$ can be obtained through a perturbation of the presentation matrix (see [93]). We can use this correspondence to study properties of codimension two Cohen-Macaulay varieties through their presentation matrix. This is the approach of Frühbis-Krüger, Pereira and Ruas.

In order to introduce the notion of EIDS and relate it with the classical approach of singularity theory, let us recall some concepts in this field.

Let $\mathscr{R}$ be the group of coordinate changes (on the source) in $\left(\mathbb{C}^{r}, 0\right)$. We denote $G L_{i}\left(\mathscr{O}_{r}\right)$ the group of invertible matrices of size $i \times i$ with entries in the local ring $\mathscr{O}_{r}$. Consider the group $\mathscr{H}:=G L_{p}\left(\mathscr{O}_{r}\right) \times G L_{n}\left(\mathscr{O}_{r}\right)$.

Given two matrices, we are interested in studying these germs according to the following equivalence relation.

Definition 1.5.2. Let $\mathscr{G}:=\mathscr{R} \ltimes \mathscr{H}$ be the semi-direct product of $\mathscr{R}$ and $\mathscr{H}$. We say that two germs $F_{1}, F_{2} \in \operatorname{Mat}_{(n, p)}\left(\mathscr{O}_{r}\right)$ are $\mathscr{G}$-equivalent if there exist $(\phi, R, L) \in \mathscr{G}$ such that $F_{1}=$ $L^{-1}\left(\phi^{*} F_{2}\right) R$.

It is not difficult to see that $\mathscr{G}$ is one of Damon's geometric subgroups of $\mathscr{K}$ (see [83]), hence as a consequence of Damon's result ([27]) we can use the techniques of singularity theory, for instance, those concerning finite determinacy. The notions of $\mathscr{G}$-equivalence and $\mathscr{K}_{\Delta}$-equivalence, where $\Delta$ consists of the subvariety of matrices of rank less than the maximal rank [27], coincide for finitely determined germs (see [17]).

The next result is a Geometric Criterion of Finite Determinacy for families of $n \times p$ matrices and was proved by Pereira in her Ph. D. thesis.

Theorem 1.5.3. (Geometric Criterion of Finite Determinacy, [83] Theorem 2.4.1) A represen-

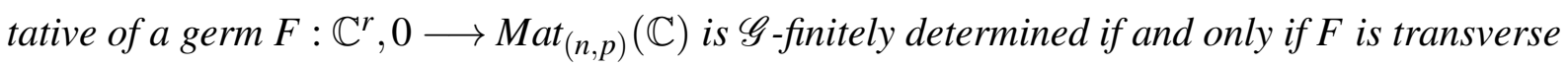
to the strata of the rank stratification of $\operatorname{Mat}_{(n, p)}(\mathbb{C})$ outside the origin. 
It follows that if $F$ is a $n \times p$ matrix with entries in the maximal ideal of $\mathscr{O}_{r}$, defining an isolated singularity, then $F$ is $\mathscr{G}$-finitely determined. Moreover if $F$ is $\mathscr{G}$-finitely determined, then the germ of $X$ at a singular point is holomorphic to either the product of $\Sigma_{t}$ with an affine space or a transverse slice of $\Sigma_{t}$. This motivates the following definition ([30]):

Definition 1.5.4. A point $x \in X=F^{-1}\left(\Sigma_{t}\right)$ is called essentially non-singular if, at the point $x$, the map $F$ is transversal to the corresponding stratum of the variety $\Sigma_{t}$. A germ $(X, 0) \subseteq\left(\mathbb{C}^{r}, 0\right)$ of a determinantal variety has an essentially isolated singular point at the origin (or is an essentially isolated determinantal singularity: EIDS) if it has only essentially non-singular points in a punctured neighborhood of the origin in $X$.

If $X=F^{-1}\left(\Sigma_{t}\right)$ then a perturbation of $X$ is obtained by perturbing the entries of $F$. This yields an unfolding of $F$, and if $X$ is an EIDS then happens to also give a deformation of $X$ which is transverse to the strata of $\operatorname{Hom}\left(\mathbb{C}^{n}, \mathbb{C}^{p}\right)$.

In the particular case where $X$ is Cohen-Macaulay of codimension 2, it is a consequence of the Auslander-Buchsbaum formula and the Hilbert-Burch Theorem that any deformation of $X$ can be given as a perturbation of the presentation matrix (see [38]). Therefore we can study these varieties and their deformations using their representation matrices. We can express the normal module $N(X)$, and the space of the first order deformations $T_{X}^{1}$, in terms of matrices, hence we can treat the base of the semi-universal deformation using matrix representation and we can express the normal module $N(X)$ in terms of matrices.

\subsection{Bi-Lipschitz geometry and Lipschitz stratifications}

In this section we follow the lines of [70] for an understanding of the bi-Lipschitz geometry.

Let $M$ and $N$ be metric spaces.

Definition 1.6.1. A map $f: M \rightarrow N$ is called Lipschitz if there exists a constant $C>0$ such that

$$
d(f(x), f(y)) \leq C d(x, y),
$$

for all $x, y \in M$.

A homeomorphism $f: M \rightarrow N$ is called bi-Lipschitz if $f$ is Lipschitz and the inverse $f^{-1}: N \rightarrow M$ is Lipschitz, as well.

In this case we say that $f$ is a bi-Lipschitz equivalence and that $M$ and $N$ are bi-Lipschitz equivalent.

In order to understand the bi-Lipschitz equisingularity, we recall here some important notions developed by Mostowski and Parusiński. 
We denote $\mathbb{K}$ the field $\mathbb{R}$ or $\mathbb{C}$.

Definition 1.6.2. A filtration of a subset $X \subseteq \mathbb{K}^{n}$ is a sequence of subsets of $X$

$$
X=X^{d_{1}} \supset X^{d_{2}} \supset \ldots \supset X^{d_{l}} \neq \emptyset
$$

with $d_{1}>d_{2}>\ldots>d_{l}$, and $X_{\text {sing }}^{d_{i}} \subseteq X^{d_{i+1}}, \forall i \in\{1, \ldots, l\}$. The superscripts $d_{i}$ denote dimensions, the $X^{d_{i}}$ are called skeletons, the manifolds $X^{d_{i}}:=X^{d_{i}}-X^{d_{i+1}}$ strata and the collection $\left\{\dot{X}^{d_{i}}\right\}$ is called a stratification of $X$.

The stratification $\left\{\stackrel{\circ}{X}^{d_{i}}\right\}$ is called weakly Lipschitz if for every $i$, for all point $p \in \dot{X}^{d_{i}}$ and vector $v \in T_{p} \dot{X}^{d_{i}}$, there is a Lipschitz vector field $\tilde{v}$ defined in a neighborhood of $p$ in $\mathbb{K}^{n}$ such that $\tilde{v}(p)=v$ and the local flow $v_{t}$ generated by $v$ satisfies $v_{t}(X) \subseteq X$.

The weakly Lipschitz stratifications have the following property: if $v$ is a Lipschitz vector field defined in a neighborhood of a point $p \in \check{X}^{d_{i}}$ in $\dot{X}^{d_{i}}$, which is tangent to $\dot{X}^{d_{i}}$, then $v$ extends to a Lipschitz vector field defined in a neighborhood of $p$ in $\mathbb{K}^{n}$, whose local flow preserves $X$.

Definition 1.6.3. Under the above notation, we say that $X$ is locally Lipschitz equisingular along a stratum $\stackrel{\circ}{\mathbf{X}}^{\mathbf{d}_{\mathbf{i}}}$ if for every $p \in \stackrel{\circ}{X}^{d_{i}}$ there is a neighborhood $U_{0}$ of $p$ in $\stackrel{\circ}{X}^{d_{i}}$, a tubular neighborhood $U$ of $U_{0}$ in $\mathbb{K}^{n}$ with a projection $\pi: U \rightarrow U_{0}$, and a bi-Lipschitz homeomorphism

$$
h: U \rightarrow U_{0} \times H_{0},
$$

where $H$ is the complement of $T_{p} U_{0}$ in $T_{p} \mathbb{K}^{n}=\mathbb{K}^{n}$, i.e,

$$
T_{p} \mathbb{K}^{n}=T_{p} U_{0} \oplus H
$$

$H_{0}$ is a neighborhood of $p$ in $H$, such that

1. $p r \circ h=\pi$;

2. $h(U \cap X)=U_{0} \times\left(H_{0} \cap X\right)$,

where pr: $U_{0} \times H_{0} \rightarrow U_{0}$ is the projection.

It is clear that $X$ is locally Lipschitz equisingular along every stratum of a weakly Lipschitz stratification. The existence of weakly Lipschitz stratifications is proved in [72].

By $\operatorname{dist}(\star, X)$ we mean the function of distance to $X$. If $X=\emptyset$ we set $\operatorname{dist}(\star, \emptyset) \equiv 1$. For any linear operator $A$ defined on $\mathbb{K}^{n}$, we define the norm of $A$ as

$$
\|A\|=\sup \left\{\|A(v)\| \mid v \in \mathbb{K}^{n},\|v\|=1\right\}
$$

For $q \in \dot{X}^{d_{j}}$, let $P_{q}: \mathbb{K}^{n} \rightarrow T_{q} \dot{X}^{d_{j}}$ be the orthogonal projection onto the tangent space of $\stackrel{\circ}{X}^{d_{j}}$ at $q$, and let $P_{q}^{\perp}=I d_{\mathbb{K}^{n}}-P_{q}: \mathbb{K}^{n} \rightarrow T_{q}^{\perp} \stackrel{\circ}{X}^{d_{j}}$ be the orthogonal projection onto the normal space. 
For technical reasons we replace the distance functions $\operatorname{dist}\left(q, X^{d_{j}}\right)$ by semianalytic functions (i.e, continuous functions with semianalytic graphs) such that

$$
\frac{1}{2 n} \operatorname{dist}\left(q, X^{d_{j}}\right) \leq \rho_{j}(q) \leq 2 n \operatorname{dist}\left(q, X^{d_{j}}\right) .
$$

For a proof of the existence of these functions $\rho_{j}$ 's see [67].

If $X^{d_{j}}$ 's are algebraic then the distance functions themselves can be used [28].

Definition 1.6.4. (Chain) Let $c_{0}$ be a fixed constant, $c_{0} \geq 2 n$. A $\mathbf{c}_{0}$-chain (or chain) for a point $q \in \dot{X}^{d_{j}}$ is a strictly increasing sequence of indices $j=j_{1}, j_{2}, \ldots, j_{r}=l$ and a sequence of points $q_{j_{s}} \in \dot{X}^{d_{j_{s}}}$ such that $j_{1}=j, q_{j_{1}}=q$ and each $j_{s}(s \geq 2)$ is the smallest integer greater than $j_{s-1}$ for which $\rho_{j_{s}-1}(q) \geq 2 c_{0}^{2} \rho_{j_{s}}(q)$, and $\left\|q-q_{j_{s}}\right\| \leq c_{0} \rho_{j_{s}}(q)$.

Now we are able to recall the following definition (see [80]).

Definition 1.6.5. (Mostowski's conditions for Lipschitz, stratifications) We call a stratification $\left\{\AA^{d_{i}}\right\}$ a Lipschitz stratification if for some constant $C>0$, every $c_{0}$-chain $q=q_{j_{1}}, q_{j_{2}}, \ldots, q_{j_{r}}$ and every $k \in\{1, \ldots, r\}$ we have:

1. $\left\|P_{q}^{\perp} \circ P_{q_{j_{2}}} \circ \ldots \circ P_{q_{j_{k}}}\right\| \leq C \frac{\left\|q-q_{j_{2}}\right\|}{\operatorname{dist}\left(q, X^{d_{j_{k-1}}}\right)}$.

If $q^{\prime} \in \stackrel{\circ}{X}^{d_{j}}$ such that $\left\|q-q^{\prime}\right\| \leq \frac{\operatorname{dist}\left(q, X^{d_{j-1}}\right)}{2 c_{0}}$ then

2. $\left\|\left(P_{q}-P_{q^{\prime}}\right) \circ P_{q_{j_{2}}} \circ \ldots \circ P_{q_{j_{k}}}\right\| \leq C \frac{\left\|q-q^{\prime}\right\|}{\operatorname{dist}\left(q, X^{d_{j_{k-1}}}\right)}$;

3. $\left\|P_{q}-P_{q^{\prime}}\right\| \leq C \frac{\left\|q-q^{\prime}\right\|}{\operatorname{dist}\left(q, X^{d_{j-1}}\right)}$.

Example 1.6.6. Consider the cone $X$ given by the equation $z^{2}=x^{2}+y^{2}$. If we take $X^{2}:=X$ and $X^{0}:=(0,0,0)$ then $\left\{X^{0}, X^{2}-X^{0}\right\}$ is a stratification of the cone $X$ which satisfies the three above Mostowski conditions.

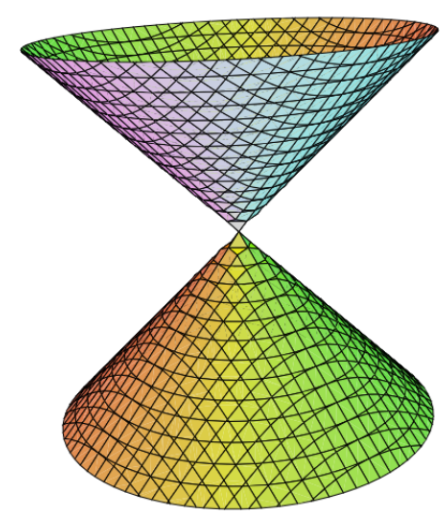

Figure 3 - Cone 
An alternate definition of Lipschitz stratification is given in [70].

Definition 1.6.7. We call a stratification $\left\{\dot{X}^{d_{i}}\right\}$ a Lipschitz stratification if for some constant $C>0$, for every compact set $F$ such that

$$
X^{d_{i+1}} \subseteq F \subseteq X^{d_{i}}
$$

and every Lipschitz vector field $v$, defined on $F$ and tangent to strata (i.e $v(x) \in T_{x} \dot{X}^{d_{j}}$ for all $x \in \dot{X}^{d_{j}}, j>i$, or $\left.x \in \dot{X}^{d_{i}} \cap F\right)$, there exists a Lipschitz vector field $\tilde{v}$, defined on $\mathbb{K}^{n}$, tangent to strata and extending $v$, such that the best Lipschitz constant for $\tilde{v}$ is less than or equal to $C$.

We state the main result of this section, due to Mostowski.

Theorem 1.6.8. ([72] Proposition 1.2) Every complex analytic set admits a complex analytic Lipschitz stratification. Moreover such Lipschitz stratification are locally bi-Lipschitz trivial along strata

In [78], Parusiński obtained the above theorem for analytic real varieties. After, he generalized this result for subanalytic sets.

Theorem 1.6.9. ([82] Theorem 1.4) Every subanalytic set admits a subanalytic Lipschitz stratification. Moreover such Lipschitz stratification are locally bi-Lipschitz trivial along strata

There is a construction of a Lipschitz stratification of an analytic hypersurface in [72], but it was not made canonically. Hence, one important thing to investigate is when it is possible to construct a canonical vector field satisfying the previous Lipschitz conditions.

Example 1.6.10. ([101] Example 5.1) Consider $\mathbb{R}^{3}$ with coordinates $x, y$ and $z$. For each $z$ consider $X_{z}=\{y=0\} \cup\left\{\left(x, x^{z}, z\right)\right\}$. Then the bi-Lipschitz type of $X_{z}$ and $X_{z^{\prime}}$ are different if $z \neq z^{\prime}$, both greater than 1 . Let us to verify this for natural numbers $n \neq m$. For each $n$ consider the map-germ $f_{n}:(\mathbb{R}, 0) \rightarrow(\mathbb{R}, 0)$ given by $f_{n}(x)=x^{n}$. Thus $f_{n}$ is finitely $C^{0}$ - $\mathscr{K}$-determined map germs. Since $\operatorname{deg} f_{n} \neq \operatorname{deg} f_{m}$ whenever $n \neq m$ then by Theorem 3.1 of [21] $f_{n}$ and $f_{m}$ are not topologically $\mathscr{K}$-equivalent if $n \neq m$. In particular, $f_{n}$ and $f_{m}$ are not bi-Lipschitz $\mathscr{K}$-equivalent for $n \neq m$, and therefore the fibers $X_{n}$ and $X_{m}$ have not the same bi-Lipschitz type. Hence, there is no bi-Lipschitz trivial stratification in this case.

For more results toward the contact equivalence, see [50, 96].

Example 1.6.11. ([59] Example 1.5) This is a semialgebraic example due to Satoshi Koike, showing that $(w)$ - regularity does not imply local bi-Lipschitz triviality. Let $X=\left\{y^{2}=z^{2} x^{2}+\right.$ $\left.x^{3}, x \geq 0\right\}$ in $\mathbb{R}^{3}$. Let $Y$ be the z-axis. Then the pair $(X-Y, Y)$ is $(w)$-regular, but the bi-Lipschitz type of the germ of $X$ at point $(0,0,0) \in Y$ is different from the bi-Lipschitz type of the germ of $X$ at points $(0,0, z) \in Y$ if $z \neq 0$. 


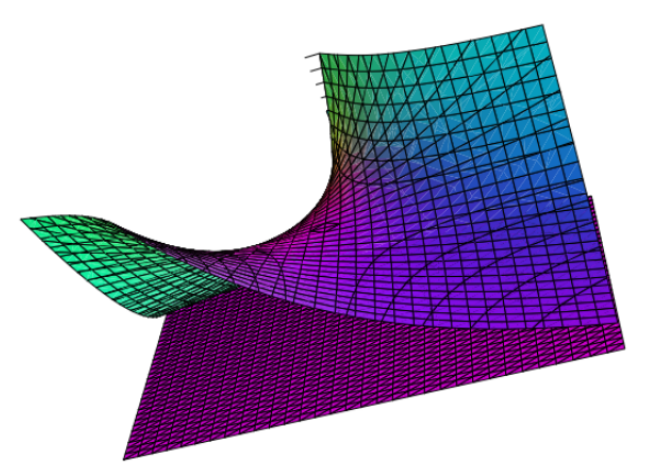

Figure $4-\{y=0\} \cup\left\{y=x^{z}\right\}, z \geq 0$

In fact, suppose that $X_{0}=\left\{y^{2}=x^{3}\right\}$ and $X_{z}=\left\{y^{2}=z^{2} x^{2}+x^{3}\right\}$ have the same bi-Lipschitz type, with $z \neq 0$. In particular $X_{0}$ and $X_{z}$ are homeomorphic, hence $X_{0}-\{0\}$ and $X_{z}-\{0\}$ as well, which is a contradiction because these sets have 2 and 3 connected components, respectively.

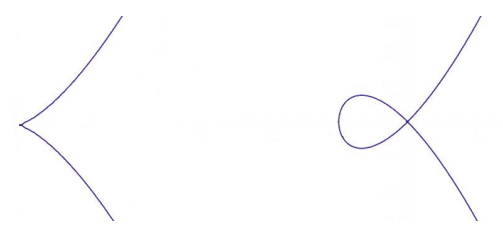

Figure 5 - Cusp and Node 
CHAPTER

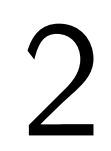

\section{THE DOUBLE OF A MODULE}

In order to deal with bi-Lipschitz equisingularity in a family of hypersurfaces and to get algebraic infinitesimal Lipschitz conditions, Gaffney defined in $[39,40]$ the double of an ideal in a quite natural way. In this chapter we extend this notion for modules and we generalize several results of [39].

\subsection{The double of a module and basic properties}

Let $X \subseteq \mathbb{C}^{n}$ be an analytic space. Consider the projection maps

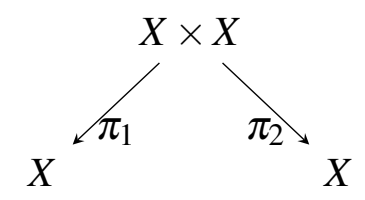

where $\pi_{i}$ is the projection onto the $i^{\text {th }}$-factor, for every $i \in\{1,2\}$.

Definition 2.1.1. Let $h \in \mathscr{O}_{X, x}^{p}$. The double of $h$ is defined as the element

$$
h_{D}:=\left(h \circ \pi_{1}, h \circ \pi_{2}\right) \in \mathscr{O}_{X \times X,(x, x)}^{2 p} .
$$

The double of a submodule $M \subseteq \mathscr{O}_{X, x}^{p}$ is denoted by $M_{D}$, and is defined as the $\mathscr{O}_{X \times X,(x, x)^{-}}$ submodule of $\mathscr{O}_{X \times X,(x, x)}^{2 p}$ generated by $\left\{h_{D} \mid h \in M\right\}$.

Let $\mathscr{M}$ be a sheaf of $\mathscr{O}_{X}$-submodules of $\mathscr{O}_{X}^{p}$. We can assume that $\mathscr{M}$ is finitely generated by global sections. If $h \in \mathscr{O}_{X}^{p}(X)$ is a global section, given an arbitrary point $\left(x, x^{\prime}\right) \in X \times X$, we can consider $h_{D} \in \mathscr{O}_{X \times X,\left(x, x^{\prime}\right)}^{2 p}$, the double of $h$, as the germ of $\left(h \circ \pi_{1}, h \circ \pi_{2}\right) \in \mathscr{O}_{X \times X}^{2 p}(X \times X)$ at the point $\left(x, x^{\prime}\right)$. Thus, we define the double of the sheaf $\mathscr{M}$, denoted $\mathscr{M}_{D}$, as the $\mathscr{O}_{X \times X^{-}}$ submodule of $\mathscr{O}_{X \times X}^{2 p}$ generated by $\left\{h_{D} \mid h \in \mathscr{M}(X)\right.$ is a global section $\}$. Unless we say the 
opposite, we denote by $M$ the stalk of $\mathscr{M}$ at an arbitrary point of $X$ and $M_{D}$ the stalk of $\mathscr{M}_{D}$ in an arbitrary point of $X \times X$. If we take the germ of $h$ at a point, we use the same notation, if there is no confusion. For any sheaf of $\mathscr{O}_{X}$-modules $\mathscr{F}$, the notation $h \in \mathscr{F}$ means that $h$ is a global section of $\mathscr{F}$.

We want to recover some results which are true in the ideal case, i.e, when $p=1$ and $M=I$ is an ideal. We start by obtain a set of generators for $M_{D}$ from a set of generators of $M$.

Consider $z_{1}, \ldots, z_{n}$ the coordinates on $\mathbb{C}^{n}$.

Lemma 2.1.2. Under the above notation, we have the following properties:

a) $(\alpha h)_{D}=-\left(0_{\mathscr{O}_{X \times X}^{p}},\left(\alpha \circ \pi_{1}-\alpha \circ \pi_{2}\right)\left(h \circ \pi_{2}\right)\right)+\left(\alpha \circ \pi_{1}\right) h_{D}$, for all $\alpha \in \mathscr{O}_{X}$ and $h \in \mathscr{O}_{X}^{p}$;

b) $\left(0_{\mathscr{O}_{X \times X}^{p}},\left(\alpha \circ \pi_{1}-\alpha \circ \pi_{2}\right)\left(h \circ \pi_{2}\right)\right) \in M_{D}$, for all $h \in M$ and $\alpha \in \mathscr{O}_{X}$;

c) $\alpha \circ \pi_{1}-\alpha \circ \pi_{2} \in I(\Delta(X))=\left(z_{1} \circ \pi_{1}-z_{1} \circ \pi_{2}, \ldots, z_{n} \circ \pi_{1}-z_{n} \circ \pi_{2}\right)$, for all $\alpha \in \mathscr{O}_{X}$;

d) $(g+h)_{D}=g_{D}+h_{D}$, for all $g, h \in \mathscr{O}_{X}^{p}$;

e) $\left(0_{\mathscr{O}_{X \times X}^{p}},\left(\alpha \circ \pi_{1}-\alpha \circ \pi_{2}\right)\left(h \circ \pi_{2}\right)\right) \in \overline{\mathscr{M}_{D}}$ at $\left(x, x^{\prime}\right)$, for all $h \in \overline{\mathscr{M}_{x^{\prime}}}$ and $\alpha \in \mathscr{O}_{X}$. The analogous property holds if we look at the first $p$ coordinates instead the last.

Proof. (a) We have: $(\alpha h)_{D}=\left((\alpha h) \circ \pi_{1},(\alpha h) \circ \pi_{2}\right)=\left(\left(\alpha \circ \pi_{1}\right)\left(h \circ \pi_{1}\right),\left(\alpha \circ \pi_{2}\right)\left(h \circ \pi_{2}\right)\right)$ $=-\left(0_{\mathscr{O}_{X \times X}^{p}},\left(\alpha \circ \pi_{1}-\alpha \circ \pi_{2}\right)\left(h \circ \pi_{2}\right)\right)+\left(\left(\alpha \circ \pi_{1}\right)\left(h \circ \pi_{1}\right),\left(\alpha \circ \pi_{1}\right)\left(h \circ \pi_{2}\right)\right)$ $=-\left(0_{\mathscr{O}_{X \times X}^{p}}^{p},\left(\alpha \circ \pi_{1}-\alpha \circ \pi_{2}\right)\left(h \circ \pi_{2}\right)\right)+\left(\alpha \circ \pi_{1}\right) h_{D}$.

(b) Since $h \in M$ then $\alpha h \in M$, so $h_{D} \in M_{D}$ and $(\alpha h)_{D} \in M_{D}$. Thus, by (a) we have that $\left(0_{\mathscr{O}_{X \times X}^{p}},\left(\alpha \circ \pi_{1}-\alpha \circ \pi_{2}\right)\left(h \circ \pi_{2}\right)\right)=\left(\alpha \circ \pi_{1}\right) h_{D}-(\alpha h)_{D} \in M_{D}$.

(c) Obviously $\alpha \circ \pi_{1}-\alpha \circ \pi_{2}$ vanishes on the diagonal of $X$, which finishes the proof of (c).

(d) Notice that: $(g+h)_{D}=\left((g+h) \circ \pi_{1},(g+h) \circ \pi_{2}\right)=\left(g \circ \pi_{1}+h \circ \pi_{1}, g \circ \pi_{2}+h \circ \pi_{2}\right)=$ $\left(g \circ \pi_{1}, g \circ \pi_{2}\right)+\left(h \circ \pi_{1}, h \circ \pi_{2}\right)=g_{D}+h_{D}$.

(e) Let $\phi:(\mathbb{C}, 0) \rightarrow\left(X \times X,\left(x, x^{\prime}\right)\right)$ be an arbitrary analytic curve, $\phi=\left(\phi_{1}, \phi_{2}\right)$. Since $h \in \overline{\mathscr{M}_{x^{\prime}}}$ then we can write

$$
h \circ \phi_{2}=\sum_{l} \alpha_{l}\left(v_{l} \circ \phi_{2}\right)
$$

with $\alpha_{l} \in \mathscr{O}_{\mathbb{C}, 0}$ and $v_{l} \in \mathscr{M}_{x^{\prime}}$, for all $l$. So we have

$\left(0,\left(\alpha \circ \pi_{1}-\alpha \circ \pi_{2}\right)\left(h \circ \pi_{2}\right)\right) \circ \phi=\left(0,\left(\alpha \circ \phi_{1}-\alpha \circ \phi_{2}\right)\left(h \circ \phi_{2}\right)\right)$

$=\sum_{l} \alpha_{l}\left(0,\left(\alpha \circ \phi_{1}-\alpha \circ \phi_{2}\right)\left(v_{l} \circ \phi_{2}\right)\right)=\sum_{l} \alpha_{l}(\underbrace{\left(0,\left(\alpha \circ \pi_{1}-\alpha \circ \pi_{2}\right)\left(v_{l} \circ \pi_{2}\right)\right)}_{\in \mathscr{M}_{D}} \circ \phi) \in \phi^{*}\left(\mathscr{M}_{D}\right)$. Hence,

$\left(0,\left(\alpha \circ \pi_{1}-\alpha \circ \pi_{2}\right)\left(h \circ \pi_{2}\right)\right) \in \overline{\mathscr{M}_{D}}$. 
The next proposition gives a set of generators of $M_{D}$, from a known set of generators of $M$, which will be useful in the proof of many results in this section and in the section 2.2.

Proposition 2.1.3. Suppose that $M$ is generated by $\left\{h_{1}, \ldots, h_{r}\right\}$. Then, the following sets are generators of $M_{D}$ :

a) $\mathscr{B}=\left\{\left(h_{1}\right)_{D}, \ldots,\left(h_{r}\right)_{D}\right\} \cup\left\{\left(0_{\mathscr{O}_{X \times X}^{p}},\left(z_{i} \circ \pi_{1}-z_{i} \circ \pi_{2}\right)\left(h_{j} \circ \pi_{2}\right)\right) \mid i \in\{1, \ldots, n\}\right.$ and $j \in\{1, \ldots, r\}\}$;

b) $\mathscr{B}^{\prime}=\left\{\left(h_{1}\right)_{D}, \ldots,\left(h_{r}\right)_{D}\right\} \cup\left\{\left(\left(z_{i} \circ \pi_{1}-z_{i} \circ \pi_{2}\right)\left(h_{j} \circ \pi_{1}\right), 0_{\mathscr{O}_{X \times X}^{p}}\right) \mid i \in\{1, \ldots, n\}\right.$ and $j \in\{1, \ldots, r\}\}$;

c) $\mathscr{B}^{\prime \prime}=\left\{\left(h_{1}\right)_{D}, \ldots,\left(h_{r}\right)_{D}\right\} \cup\left\{\left(z_{i} h_{j}\right)_{D} \mid i \in\{1, \ldots, n\}\right.$ and $\left.j \in\{1, \ldots, r\}\right\}$.

Proof. (a) Let $N$ be the submodule of $\mathscr{O}_{X \times X}^{2 p}$ generated by $\mathscr{B}$. By Lemma 2.1.2 (b) we have that $N \subseteq M_{D}$. Now, in order to verify that $M_{D} \subseteq N$, it is enough to check that $h_{D} \in N, \forall h \in M$. Indeed, if $h \in M$ we can write $h=\sum_{j=1}^{r} \alpha_{j} h_{j}$, for some $\alpha_{j} \in \mathscr{O}_{X}$. By Lemma 2.1.2 (a) and (d) we have that $h_{D}=\left(\sum_{j=1}^{r} \alpha_{j} h_{j}\right)_{D}=\sum_{j=1}^{r}\left(\alpha_{j} h_{j}\right)_{D}$ $=\left(\sum_{j=1}^{r}\left(\alpha_{j} \circ \pi_{1}\right)\left(h_{j}\right)_{D}\right)-\left(\sum_{j=1}^{r}\left(0_{\mathscr{O}_{X \times X}^{p}},\left(\alpha_{j} \circ \pi_{1}-\alpha_{j} \circ \pi_{2}\right)\left(h_{j} \circ \pi_{2}\right)\right)\right)$. Clearly the first sum is in $N$. By Lemma 2.1.2 (c) we have that each $\alpha_{j} \circ \pi_{1}-\alpha_{j} \circ \pi_{2}$ belongs to the ideal $I(\Delta(X))$, so the second sum is in $N$, which finishes the proof.

(b) This is completely analogous to the item (a).

(c) We use (a). Let $N$ be the submodule of $\mathscr{O}_{X \times X}^{2 p}$ generated by $\mathscr{B}^{\prime \prime}$. For all $j \in\{1, \ldots, r\}$ and $i \in\{1, \ldots, n\}$ we have

$$
\left(z_{i} h_{j}\right)_{D}=\left(z_{i} \circ \pi_{1}\right)\left(h_{j}\right)_{D}-\left(0_{\mathscr{O}_{X \times X}^{p}},\left(z_{i} \circ \pi_{1}-z_{i} \circ \pi_{2}\right)\left(h_{j} \circ \pi_{2}\right)\right) \in M_{D},
$$

by the previous lemma. Hence, $N \subseteq M_{D}$. Now, in order to check that $M_{D} \subseteq N$, it suffices to verify that all the generators of $M_{D}$ given in (a) are in $N$. We already have $\left(h_{j}\right)_{D} \in N$, for all $j \in\{1, \ldots, r\}$. Also, for all $j$ and $i$ we have

$$
\left(0_{\mathscr{O}_{X \times X}^{p}},\left(z_{i} \circ \pi_{1}-z_{i} \circ \pi_{2}\right)\left(h_{j} \circ \pi_{2}\right)\right)=\left(z_{i} \circ \pi_{1}\right)\left(h_{j}\right)_{D}-\left(z_{i} h_{j}\right)_{D} \in N
$$

which finishes the proof.

We can develop the same notion for the double in the family case. Suppose that $X \subseteq \mathbb{C}^{n+k}$ is an analytic space and let $Y=0 \times \mathbb{C}^{k} \subseteq X$. Identifying $Y=0 \times \mathbb{C}^{k}=\mathbb{C}^{k}$ we have that $X \subseteq \mathbb{C}^{n} \times Y$. Let $\mathbf{p}: X \subseteq \mathbb{C}^{n} \times Y \rightarrow Y$ be the projection, $X \times \underset{Y}{X}$ the fibered product relative to the projection onto $Y$, with the projections maps $\pi_{1}, \pi_{2}: X \underset{Y}{\stackrel{Y}{X}} X \rightarrow X$. 


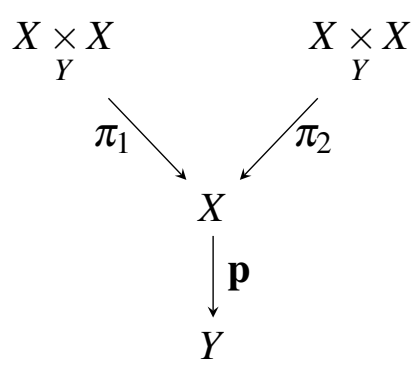

Let $h \in \mathscr{O}_{X}^{p}$. The double of $\mathbf{h}$ relative to $\mathbf{Y}$ is defined by

$$
h_{D, Y}:=h_{D}:=\left(h \circ \pi_{1}, h \circ \pi_{2}\right) \in \mathscr{O}_{X \times X}^{2 p} .
$$

In the same way, the double of a sheaf of $\mathscr{O}_{X}$-submodules $\mathscr{M}$ of $\mathscr{O}_{X}^{p}$ relative to $Y$ is defined as the $\mathscr{O}_{X \times X}$-submodule of $\mathscr{O}_{X \times X}^{2 p}$ generated by $\left\{h_{D, Y} \mid h \in \mathscr{M}(X)\right.$ is a global section $\}$, and is denoted by $\mathscr{M}_{D}\left(\right.$ or $\left.\mathscr{M}_{D, Y}\right)$.

Let $z_{1}, \ldots, z_{n}, y_{1}, \ldots, y_{k}$ be the coordinates on $\mathbb{C}^{n+k}$. It is easy to see that the Lemma 2.1.2 still holds when we are working with the projections restricted to the fibered product $\underset{Y}{X} X$ and, since each $y_{l} \circ \pi_{1}-y_{l} \circ \pi_{2}$ vanishes on the fibered product, then we get the following proposition, whose proof is completely analogous to the proof of the Proposition 2.1.3.

Proposition 2.1.4. Suppose that $M$ is generated by $\left\{h_{1}, \ldots, h_{r}\right\}$. Then, the following sets are generators of $M_{D}$ relative to $Y$ :

a) $\mathscr{B}=\left\{\left(h_{1}\right)_{D}, \ldots,\left(h_{r}\right)_{D}\right\} \cup\left\{\left(0_{\mathscr{O}_{X \times X}^{p}},\left(z_{i} \circ \pi_{1}-z_{i} \circ \pi_{2}\right)\left(h_{j} \circ \pi_{2}\right)\right) \mid i \in\{1, \ldots, n\}\right.$ and $j \in\{1, \ldots, r\}\}$;

b) $\mathscr{B}^{\prime}=\left\{\left(h_{1}\right)_{D}, \ldots,\left(h_{r}\right)_{D}\right\} \cup\left\{\left(\left(z_{i} \circ \pi_{1}-z_{i} \circ \pi_{2}\right)\left(h_{j} \circ \pi_{1}\right), 0_{\mathscr{O}_{X \times X}^{p}}\right) \mid i \in\{1, \ldots, n\}\right.$ and $j \in\{1, \ldots, r\}\}$

c) $\mathscr{B}^{\prime \prime}=\left\{\left(h_{1}\right)_{D}, \ldots,\left(h_{r}\right)_{D}\right\} \cup\left\{\left(z_{i} h_{j}\right)_{D} \mid i \in\{1, \ldots, n\}\right.$ and $\left.j \in\{1, \ldots, r\}\right\}$.

Example 2.1.5. Consider the ideal $I=\left(x^{2}, y^{2}\right)$ of $\mathscr{O}_{\mathbb{C}^{2}, 0}$. Notice that $I_{D}$ has generators further the doubles of $x^{2}$ and $y^{2}$. In fact, by Proposition 2.1.3 the double $I_{D}$ is generated by $\mathscr{B}:=$ $\left\{\left(x^{2}\right)_{D},\left(y^{2}\right)_{D},\left(0,\left(x-x^{\prime}\right) x^{\prime}\right),\left(0,\left(x-x^{\prime}\right) y^{\prime}\right),\left(0,\left(y-y^{\prime}\right) x^{\prime}\right),\left(0,\left(y-y^{\prime}\right) y^{\prime}\right)\right\}$. It is easy to see that no element of $\mathscr{B}$ can be generated by the another elements of $\mathscr{B}$, i.e, $\mathscr{B}$ is a minimal set of generators of $I_{D}$. Hence, we need further elements than the doubles of $x^{2}$ and $y^{2}$ to generate the double of $I$.

Remember that for any sheaf $\mathscr{F}$ of $\mathscr{O}_{X}$-modules, the support of $\mathscr{F}$ is defined as the subset of $X$ given by

$$
\operatorname{supp}(\mathscr{F}):=\left\{x \in X \mid \mathscr{F}_{x} \neq 0\right\}
$$


If $\mathscr{G}$ is a sheaf of $\mathscr{O}_{X}$-submodules of $\mathscr{F}$, the cosupport of $\mathscr{G}$ in $\mathscr{F}$ is defined as

$$
\operatorname{cosupp}(\mathscr{G}):=\operatorname{supp}\left(\frac{\mathscr{F}}{\mathscr{G}}\right)
$$

Let us to fix some notations. Let us assume that $\mathscr{M}$ is generated by global sections $\left\{h_{1}, \ldots, h_{r}\right\}$ of $\mathscr{O}_{X}^{p}$, let $[\mathscr{M}]$ be the $p \times r$ matrix whose columns are the generators of $\mathscr{M}$, and for each $x \in X$ let $[\mathscr{M}(x)]$ be the $p \times r$ matrix whose columns are $h_{1}(x), \ldots, h_{r}(x)$.

Let $\Sigma(\mathscr{M}):=\{x \in X \mid \operatorname{rank}[\mathscr{M}(x)]<p\}$. This set is called the singular set of $\mathscr{M}$.

In the next theorem we compute the cosupport of the double $\mathscr{M}_{D}$ in $\mathscr{O}_{X \times X}^{2 p}$.

Theorem 2.1.6. Let $\mathscr{M}$ be a sheaf of $\mathscr{O}_{X}$-submodules of $\mathscr{O}_{X}^{p}$. Then

$$
\operatorname{cosupp}\left(\mathscr{M}_{D}\right)=\Delta(X) \cup(X \times \Sigma(\mathscr{M})) \cup(\Sigma(\mathscr{M}) \times X) .
$$

Proof. Let $\left(x, x^{\prime}\right) \in X \times X$ and suppose that $\left(x, x^{\prime}\right)$ is not in the $\operatorname{cosupp}\left(\mathscr{M}_{D}\right)$.

(1) In a neighborhood of this point, by Proposition 2.1.3 we can write

$$
\left(1,0, \ldots, 0,0_{\mathscr{O}_{X \times X}^{p}}\right)=\left(\sum \alpha_{i}\left(h_{i} \circ \pi_{1}, h_{i} \circ \pi_{2}\right)\right)+\left(\sum \beta_{i j}\left(\left(z_{i} \circ \pi_{1}-z_{i} \circ \pi_{2}\right)\left(h_{j} \circ \pi_{1}\right), 0_{\mathscr{O}_{X \times X}^{p}}\right)\right)
$$

for some $\alpha_{i}, \beta_{i j} \in \mathscr{O}_{X \times X}$.

If $\left(x, x^{\prime}\right) \in \Delta(X)$, applying the above equation in this point, the second sum vanishes and we get

$$
\left(1,0, \ldots, 0,0_{\mathbb{C}^{p}}\right)=\sum \alpha_{i}(x, x)\left(h_{i}(x), h_{i}(x)\right) .
$$

Notice that the right hand side of the above equation is in the diagonal of $\mathbb{C}^{p}$ and the left hand side is not, which is a contradiction. So, $\left(x, x^{\prime}\right) \notin \Delta(X)$.

(2) Suppose by contradiction that $\left(x, x^{\prime}\right) \in \Sigma(\mathscr{M}) \times X$. Then $\operatorname{rank}[\mathscr{M}(x)]<p$, so there exists $v \in \mathbb{C}^{p}$ such that $v$ is not a linear combination of $h_{1}(x), \ldots, h_{r}(x)$. Consider the element $\left(v, 0_{\mathscr{O}_{X \times X}^{p}}\right) \in \mathscr{O}_{X \times X}^{2 p}$ (thinking $v$ as a constant function). Since $\left(x, x^{\prime}\right)$ is not in the cosupport of $\mathscr{M}_{D}$ then in a neighborhood of this point, by Proposition 2.1.3 we can write

$$
\left(v, 0_{\mathscr{O}_{X \times X}^{p}}\right)=\left(\sum \alpha_{i}\left(h_{i} \circ \pi_{1}, h_{i} \circ \pi_{2}\right)\right)+\left(\sum \beta_{i j}\left(0_{\mathscr{O}_{X \times X}^{p}},\left(z_{i} \circ \pi_{1}-z_{i} \circ \pi_{2}\right)\left(h_{j} \circ \pi_{2}\right)\right)\right)
$$

for some $\alpha_{i}, \beta_{i j} \in \mathscr{O}_{X \times X}$. Now, comparing the first $p$ coordinates in above equation and applying in $\left(x, x^{\prime}\right)$ we have that $v=\sum \alpha_{i}\left(x, x^{\prime}\right) h_{i}(x)$, so $v$ is a linear combination of $h_{1}(x), \ldots, h_{r}(x)$, which is a contradiction. Hence, $\left(x, x^{\prime}\right) \notin \Sigma(\mathscr{M}) \times X$.

(3) Analogously we can conclude that $\left(x, x^{\prime}\right) \notin X \times \Sigma(\mathscr{M})$ by using again the Proposition 2.1.3 with the natural changes.

The three above steps prove the inclusion

$$
\operatorname{cosupp}\left(\mathscr{M}_{D}\right) \supseteq \Delta(X) \cup(X \times \Sigma(\mathscr{M})) \cup(\Sigma(\mathscr{M}) \times X)
$$


Now, let $\left(x, x^{\prime}\right) \in X \times X$ such that $x \neq x^{\prime},[\mathscr{M}(x)]$ and $\left[\mathscr{M}\left(x^{\prime}\right)\right]$ have rank $\geq p$. For each $z \in X$ denote by $[\mathscr{M}(z)]_{p}$ the $p \times p$ submatrix given by the first $p$ columns of $[\mathscr{M}(z)]$. Since $[\mathscr{M}(x)]$ and $\left[\mathscr{M}\left(x^{\prime}\right)\right]$ have rank $\geq p$, without loss of generality we can assume that $[\mathscr{M}(x)]_{p}$ and $\left[\mathscr{M}\left(x^{\prime}\right)\right]_{p}$ are invertibles. Thus, there exist $U$ and $U^{\prime}$ open subsets of $X$ containing $x$ and $x^{\prime}$, respectively, such that $[\mathscr{M}(z)]_{p}$ and $\left[\mathscr{M}\left(z^{\prime}\right)\right]_{p}$ are invertibles, $\forall\left(z, z^{\prime}\right) \in U \times U^{\prime}$. Since $x \neq x^{\prime}$ then there exists $k \in\{1, \ldots, n\}$ such that $\left(z_{k} \circ \pi_{1}-z_{k} \circ \pi_{2}\right)\left(x, x^{\prime}\right) \neq 0$, so $z_{k} \circ \pi_{1}-z_{k} \circ \pi_{2}$ is invertible in $\mathscr{O}_{X \times X,\left(x, x^{\prime}\right)}$.

Let us to prove that $\left(e_{i}, 0_{\mathscr{O}_{X \times X}^{p}}\right) \in \mathscr{M}_{D}$ at $\left(x, x^{\prime}\right), \forall i \in\{1, \ldots, p\}$. In fact, given a such $i$, consider the analytic map $\alpha: U \times U^{\prime} \rightarrow \mathbb{C}^{p}$ given by $\alpha\left(z, z^{\prime}\right):=[\mathscr{M}(z)]_{p}^{-1} \cdot e_{i}$, with $\alpha_{j}$ its coordinates functions. So, it is easy to see that $\sum_{j=1}^{p} \alpha_{j}\left(z, z^{\prime}\right) h_{j}(z)=e_{i}, \forall\left(z, z^{\prime}\right) \in U \times U^{\prime}$. Thus, in the open subset $U \times U^{\prime}$ (which contains the point $\left(x, x^{\prime}\right)$ ) we have $e_{i}=\sum_{j=1}^{p} \alpha_{j}\left(h_{j} \circ \pi_{1}\right)$, which implies that

$$
\left(e_{i}, 0_{\mathscr{O}_{X \times X}^{p}}\right)=\sum_{j=1}^{p} \alpha_{j}\left(z_{k} \circ \pi_{1}-z_{k} \circ \pi_{2}\right)^{-1} \underbrace{\left(\left(z_{k} \circ \pi_{1}-z_{k} \circ \pi_{2}\right)\left(h_{j} \circ \pi_{1}\right), 0_{\mathscr{O}_{X \times X}^{p}}\right)}_{\in \mathscr{M}_{D}}
$$

$\Rightarrow\left(e_{i}, 0_{\mathscr{O}_{X \times X}^{p}}\right) \in \mathscr{M}_{D}$

Analogously, $\left(0_{\mathscr{O}_{X \times X}^{p}}, e_{i}\right) \in \mathscr{M}_{D}$ at $\left(x, x^{\prime}\right), \forall i \in\{1, \ldots, p\}$, hence $\mathscr{M}_{D}=\mathscr{O}_{X \times X,\left(x, x^{\prime}\right)}^{2 p}$ and $\left(x, x^{\prime}\right) \notin \operatorname{cosupp}\left(\mathscr{M}_{D}\right)$.

It is easy to see this theorem still holds in the family case, replacing the corresponding sets by $X \underset{Y}{\times} \Sigma(\mathscr{M})$ and $\Sigma(\mathscr{M}) \underset{Y}{\times} X$.

Remark 2.1.7. Notice that if the singular set of $\mathscr{M}$ is empty then $\operatorname{cosupp}\left(\mathscr{M}_{D}\right)=\Delta(X)$ the diagonal of $X$ and if the singular set of $\mathscr{M}$ is the whole $X$ then cosupp $\left(\mathscr{M}_{D}\right)=X \times X$.

Example 2.1.8. Let $F: \mathbb{C}^{n} \rightarrow \mathbb{C}^{p}$ be an analytic map with $n<p$ and $X=F^{-1}(0)$. Let $J M(X)$ be the jacobian module of $X$. In this case, the rank of $J M(X)$ at any point of $X$ is less than or equal to $\min \{n, p\}=n<p$, then $\Sigma(J M(X))=X$ and $\operatorname{cosupp}\left((J M(X))_{D}\right)=X \times X$.

Example 2.1.9. Let $F: \mathbb{C}^{n} \rightarrow \mathbb{C}^{p}$ be an analytic map and suppose that $F$ is a submersion. Thus $X=F^{-1}(0)$ is a smooth submanifold of $\mathbb{C}^{n}$ of dimension $n-p$ and the singular set of the jacobian module $J M(X)$ is empty. Therefore, $\operatorname{cosupp}\left((J M(X))_{D}\right)=\Delta(X)$.

Example 2.1.10. Let us consider the cusp deformation $X=F^{-1}(0) \subseteq \mathbb{C}^{3}$ given by the equation $F(x, y, z)=x^{3}+z^{2} x^{2}-y^{2}=0$. Thus the gradient of $F$ at a point $(x, y, z)$ is $\left(3 x^{2}+2 z^{2} x,-2 y, 2 z x^{2}\right)$ and it is easy to see that the singular set of the jacobian module of $X$ is the $z$-axis. Therefore, $\operatorname{cosupp}\left((J M(X))_{D}\right)=\Delta(X) \cup(X \times(z$-axis $)) \cup((z$-axis $) \times X)$.

The next proposition generalizes the Corollary 3.4 of [39] for modules. 
Proposition 2.1.11. Let $\mathscr{M} \subseteq \mathscr{N} \subseteq \overline{\mathscr{M}}$ be $\mathscr{O}_{X}$-submodules of $\mathscr{O}_{X}^{p}$, with $X$ equidimensional. Suppose that $M_{D}$ has finite colenght in $N_{D}$ and $N_{D}$ has finite colenght in $(\bar{M})_{D}$. Then

$$
e\left(M_{D},(\bar{M})_{D}\right)=e\left(N_{D},(\bar{M})_{D}\right) \text { if and only if } \overline{M_{D}}=\overline{N_{D}}
$$

Proof. By the principle of additivity (see Theorem 6.7 of [62]), we have that

$$
e\left(M_{D},(\bar{M})_{D}\right)=e\left(M_{D}, N_{D}\right)+e\left(N_{D},(\bar{M})_{D}\right)
$$

Notice that all these multiplicities are well-defined by hypothesis. So, $e\left(M_{D},(\bar{M})_{D}\right)=e\left(N_{D},(\bar{M})_{D}\right)$ if and only if $e\left(M_{D}, N_{D}\right)=0$, which is equivalent to the equality $\overline{M_{D}}=\overline{N_{D}}$, since $X$ is equidimensional.

The following proposition and corollary will be useful later to make a relation between the saturation and the double of a module, and to work with the infinitesimal Lipschitz conditions.

Proposition 2.1.12. Let $h \in \mathscr{O}_{X}^{p}$.

a) If $h_{D} \in \overline{\mathscr{M}_{D}}$ at $\left(x, x^{\prime}\right)$ then $h \in \overline{\mathscr{M}}$ at $x$ and $x^{\prime}$;

b) If $h_{D} \in\left(\mathscr{M}_{D}\right)^{\dagger}$ at $\left(x, x^{\prime}\right)$ then $h \in \mathscr{M}^{\dagger}$ at $x$ and $x^{\prime}$.

The same result still holds in the family case.

Proof. (a) Let us to prove that $h \in \overline{\mathscr{M}}$ at $x$ (the case at $x^{\prime}$ is completely analogous). Let $\phi:(\mathbb{C}, 0) \rightarrow(X, x)$ be an arbitrary analytic curve. Define $\gamma:(\mathbb{C}, 0) \rightarrow\left(X \times X,\left(x, x^{\prime}\right)\right)$ given by $\gamma(t)=\left(\phi(t), x^{\prime}\right)$. Since $h_{D} \in \overline{\mathscr{M}}_{D}$ then $h_{D} \circ \gamma \in \mathscr{M}_{D} \circ \gamma$, so we can write

$$
h_{D} \circ \gamma=\sum_{j} \alpha_{j}\left(\left(g_{j}\right)_{D} \circ \gamma\right)
$$

with $g_{j} \in \mathscr{M}$ and $\alpha_{j} \in \mathscr{O}_{\mathbb{C}, 0}$. Since $\pi_{1} \circ \gamma=\phi$, comparing the first $p$ coordinates of the above equation, we get $h \circ \phi=\sum_{j} \alpha_{j}\left(g_{j} \circ \phi\right) \in \mathscr{M} \circ \phi$. Therefore, $h \in \overline{\mathscr{M}}$ at $x$.

(b) Let $t$ be the coordinate on $\mathbb{C}$. Let us to prove that $h \in \mathscr{M}^{\dagger}$ at $x$ (the case at $x^{\prime}$ is completely analogous). Let $\phi:(\mathbb{C}, 0) \rightarrow(X, x)$ be an arbitrary analytic curve. Define $\gamma:(\mathbb{C}, 0) \rightarrow$ $\left(X \times X,\left(x, x^{\prime}\right)\right)$ given by $\gamma(t)=\left(\phi(t), x^{\prime}\right)$. Since $h_{D} \in\left(\mathscr{M}_{D}\right)^{\dagger}$ then $h_{D} \circ \gamma \in m_{1}\left(\mathscr{M}_{D} \circ \gamma\right)$, so we can write

$$
h_{D} \circ \gamma=\sum_{j} t \alpha_{j}\left(\left(g_{j}\right)_{D} \circ \gamma\right)
$$

with $g_{j} \in \mathscr{M}$ and $\alpha_{j} \in \mathscr{O}_{\mathbb{C}, 0}$. Since $\pi_{1} \circ \gamma=\phi$, comparing the first $p$ coordinates of the above equation, we get $h \circ \phi=\sum_{j} t \alpha_{j}\left(g_{j} \circ \phi\right) \in m_{1}(\mathscr{M} \circ \phi)$. Therefore, $h \in \mathscr{M}^{\dagger}$ at $x$.

The proof in the family case is quite analogous, working on the fibered product $X \underset{Y}{X}$. 
Example 2.1.13. As in Example 1.4.4, consider $F(x, y)=x^{2}+y^{p}, p \geq 3$ odd. Denote by $X$ the plane curve defined by $F$. We have seen that if we take $q=\frac{p+1}{2}$ then $y^{q} \in \overline{J(f)}$ and $\left(y^{q}\right)_{D} \notin \overline{(J(f))_{D}}$. Hence, the converse of the Proposition 2.1.12 (a) is not true in general.

Despite the converse of the Proposition 2.1.12 (a) is not true in general, the following proposition gives a condition such that the converse holds.

Proposition 2.1.14. Let $M$ be an $\mathscr{O}_{x, x}$-submodule of $\mathscr{O}_{X, x}^{p}$ and suppose that $I_{\Delta}^{\oplus p} \oplus I_{\Delta}^{\oplus p} \subseteq \overline{M_{D}}$.

If $h \in \bar{M}$ then :

a) $\left(h \circ \pi_{1}, h \circ \pi_{1}\right) \in \overline{M_{D}}$;

b) $\left(h \circ \pi_{2}, h \circ \pi_{2}\right) \in \overline{M_{D}}$;

c) $h_{D}=\left(h \circ \pi_{1}, h \circ \pi_{2}\right) \in \overline{M_{D}}$;

d) $\left(h \circ \pi_{2}, h \circ \pi_{1}\right) \in \overline{M_{D}}$.

The same result still holds in the family case.

Proof. (a) Assume first that $h \in M$. We can write $\left(h \circ \pi_{1}, h \circ \pi_{1}\right)=h_{D}+\left(0, h \circ \pi_{1}-h \circ \pi_{2}\right)$. In this case we have that $h_{D} \in M_{D} \subseteq \overline{M_{D}}$ and by hypothesis $\left(0, h \circ \pi_{1}-h \circ \pi_{2}\right) \in 0 \oplus I_{\Delta}^{\oplus p} \subseteq \overline{M_{D}}$. Hence, $\left(h \circ \pi_{1}, h \circ \pi_{1}\right) \in \overline{M_{D}}$.

Now, let us go back to the general case. Suppose that $h \in \bar{M}$ and take an arbitrary analytic curve $\phi:(\mathbb{C}, 0) \rightarrow(X \times X,(x, x))$ written as $\phi=\left(\phi_{1}, \phi_{2}\right)$. Since $h \in \bar{M}$ then we can write $h \circ \phi_{1}=\sum_{i} \alpha_{i}\left(g_{i} \circ \phi_{1}\right)$ with $g_{i} \in M$ and $\alpha_{i} \in \mathscr{O}_{\mathbb{C}, 0}$, for all $i$. By what we have proved before, we know that $\left(g_{i} \circ \pi_{1}, g_{i} \circ \pi_{1}\right) \in \overline{M_{D}}$ for all $i$. Thus, $\left(h \circ \pi_{1}, h \circ \pi_{1}\right) \circ \phi=\left(h \circ \phi_{1}, h \circ \phi_{1}\right)$

$=\left(\sum_{i} \alpha_{i}\left(g_{i} \circ \phi_{1}\right), \sum_{i} \alpha_{i}\left(g_{i} \circ \phi_{1}\right)\right)=\sum_{i} \alpha_{i}(\underbrace{\left(g_{i} \circ \pi_{1}, g_{i} \circ \pi_{1}\right)}_{\in \overline{M_{D}}} \circ \phi) \in \phi^{*}\left(\overline{M_{D}}\right)$. Hence,

$\left(h \circ \pi_{1}, h \circ \pi_{1}\right) \in \overline{\overline{M_{D}}}=\overline{M_{D}}$.

(b) The proof is analogous to the item (a) using the fact that $I_{\Delta}^{\oplus p} \oplus 0 \subseteq \overline{M_{D}}$.

(c) We can write $h_{D}=\left(h \circ \pi_{1}, h \circ \pi_{1}\right)+\left(0, h \circ \pi_{2}-h \circ \pi_{1}\right)$. By the item (a) we already know that $\left(h \circ \pi_{1}, h \circ \pi_{1}\right) \in \overline{M_{D}}$ and since $\left(0, h \circ \pi_{2}-h \circ \pi_{1}\right) \in 0 \oplus I_{\Delta}^{\oplus p} \subseteq \overline{M_{D}}$ then $h_{D} \in \overline{M_{D}}$.

(d) Notice that $\left(h \circ \pi_{2}, h \circ \pi_{1}\right)=\left(h \circ \pi_{2}-h \circ \pi_{1}, 0\right)+\left(h \circ \pi_{1}, h \circ \pi_{1}\right)$. By the item (a) we already know that $\left(h \circ \pi_{1}, h \circ \pi_{1}\right) \in \overline{M_{D}}$ and since $\left(h \circ \pi_{2}-h \circ \pi_{1}, 0\right) \in I_{\Delta}^{\oplus p} \oplus 0 \subseteq \overline{M_{D}}$ then $\left(h \circ \pi_{2}, h \circ \pi_{1}\right) \in \overline{M_{D}}$. 
Corollary 2.1.15. Let $\mathscr{M}$ and $\mathscr{N}$ be sheaves of $\mathscr{O}_{X}$-submodules of $\mathscr{O}_{X}^{p}$.

a) If $\mathscr{M}_{D} \subseteq \overline{\mathscr{N}_{D}}$ at $\left(x, x^{\prime}\right)$ then $\mathscr{M} \subseteq \overline{\mathscr{N}}$ at $x$ and $x^{\prime}$;

b) If $\mathscr{M}_{D} \subseteq\left(\mathscr{N}_{D}\right)^{\dagger}$ at $\left(x, x^{\prime}\right)$ then $\mathscr{M} \subseteq \mathscr{N}^{\dagger}$ at $x$ and $x^{\prime}$.

The same result still holds in the family case.

Proof. (a) Suppose that $h \in \mathscr{M}$ at $x$ and $x^{\prime}$. So, $h_{D} \in \mathscr{M}_{D}$ at $\left(x, x^{\prime}\right)$, and since $\mathscr{M}_{D} \subseteq \overline{\mathscr{N}}_{D}$ at $\left(x, x^{\prime}\right)$ then $h_{D} \in \overline{\mathscr{N}_{D}}$ at $\left(x, x^{\prime}\right)$. By Proposition 2.1.12 (a) we conclude that $h \in \overline{\mathscr{N}}$ at $x$ and $x^{\prime}$.

(b) Suppose that $h \in \mathscr{M}$ at $x$ and $x^{\prime}$. So, $h_{D} \in \mathscr{M}_{D}$ at $\left(x, x^{\prime}\right)$, and since $\mathscr{M}_{D} \subseteq\left(\mathscr{N}_{D}\right)^{\dagger}$ at $\left(x, x^{\prime}\right)$ then $h_{D} \in\left(\mathscr{N}_{D}\right)^{\dagger}$ at $\left(x, x^{\prime}\right)$. By Proposition 2.1.12 (b) we conclude that $h \in \mathscr{N}^{\dagger}$ at $x$ and $x^{\prime}$.

Looking to the Example 2.1.13, we conclude that the converse of the Corollary 2.1.15 (a) also is not true in general.

In the next corollary, we prove that the double of a module is contained in the integral closure of the double of another module, provided the doubles of the generators are.

Corollary 2.1.16. Let $M$ and $N$ be $\mathscr{O}_{X, x}$-submodules of $\mathscr{O}_{X, x}^{p}$. Suppose that $M$ is generated by $\left\{g_{1}, \ldots, g_{r}\right\}$ and let $M_{D}^{\prime}$ be the submodule of $\mathscr{O}_{X \times X,(x, x)}^{2 p}$ generated by $\left\{\left(g_{1}\right)_{D}, \ldots,\left(g_{r}\right)_{D}\right\}$. If $M_{D}^{\prime} \subseteq \overline{N_{D}}$ then $M_{D} \subseteq \overline{N_{D}}$. The same result still holds in the family case.

Proof. Let us prove that $\left(0,\left(z_{i} \circ \pi_{1}-z_{i} \circ \pi_{2}\right)\left(g_{j} \circ \pi_{2}\right)\right) \in \overline{N_{D}}$, for all $i \in\{1, \ldots, n\}$ and $j \in\{1, \ldots, r\}$. In fact, let $i$ and $j$ be arbitrary. Since $\left(g_{j}\right)_{D} \in M_{D}^{\prime} \subseteq \overline{N_{D}}$ then by Proposition 2.1.12 (a) we have that $g_{j} \in \bar{N}$. By Lemma 2.1.2 (e) we conclude that $\left(0,\left(z_{i} \circ \pi_{1}-z_{i} \circ \pi_{2}\right)\left(g_{j} \circ \pi_{2}\right)\right) \in \overline{N_{D}}$.

Therefore, all the generators of $M_{D}$ belong to $\overline{N_{D}}$ and so $M_{D} \subseteq \overline{N_{D}}$.

In the next theorem we compute the generic rank of the double of a module.

Theorem 2.1.17. Let $(X, x)$ be an irreducible analytic complex germ of dimension $d \geq 1$ and $M \subseteq \mathscr{O}_{X, x}^{p}$ a submodule of generic rank $k$. Then $M_{D}$ has generic rank $2 k$ at $(x, x)$.

The same result still holds in the family case.

Proof. Let $\left\{g_{1}, \ldots, g_{r}\right\}$ be a set of generators of $M$. We can write $[M]=\left[\begin{array}{ccc}\mid & & \mid \\ g_{1} & \ldots & g_{r} \\ \mid & & \mid\end{array}\right]=$ $\left[\begin{array}{ccc}-- & v_{1} & -- \\ & \vdots & \\ -- & v_{p} & --\end{array}\right]$, where $v_{1}, \ldots, v_{p} \in \mathscr{O}_{X, x}^{r}$ are the rows of $[M]$. Since $M$ has generic rank $k$ then 
we can choose $k$ rows of $[M]$ which are $\mathscr{O}_{X, x}$-linearly independent. Without loss of generality, we can suppose that $v_{1}, \ldots, v_{k}$ are linearly independent.

For each $l \in\{1, \ldots, p\}$, let

$$
w_{l}:=\left(v_{l} \circ \pi_{1}, 0_{\mathscr{O}_{X, x}^{n . r}}\right)
$$

and

$$
\bar{w}_{l}:=\left(v_{l} \circ \pi_{2},\left(z_{1} \circ \pi_{1}-z_{1} \circ \pi_{2}\right)\left(v_{l} \circ \pi_{2}\right), \ldots,\left(z_{n} \circ \pi_{1}-z_{n} \circ \pi_{2}\right)\left(v_{l} \circ \pi_{2}\right)\right) .
$$

Thus, $w_{1}, \ldots, w_{p}, \bar{w}_{1}, \ldots, \bar{w}_{p}$ are the rows of the matrix $\left[M_{D}\right]$ given by the generators of $M_{D}$ induced by the generators of $M$.

Claim 1: $\left\{\mathbf{w}_{\mathbf{1}}, \ldots, \mathbf{w}_{\mathbf{k}}, \overline{\mathbf{w}}_{\mathbf{1}}, \ldots, \overline{\mathbf{w}}_{\mathbf{k}}\right\}$ is $\mathscr{O}_{X \times X,(x, x)}$-linearly independent.

In fact, let $\alpha_{1}, \ldots, \alpha_{k}, \beta_{1}, \ldots, \beta_{k} \in \mathscr{O}_{X \times X,(x, x)}$ be arbitrary such that

$$
\sum_{l=1}^{k} \alpha_{l} w_{l}+\sum_{l=1}^{k} \beta_{l} \bar{w}_{l}=0_{\mathscr{O}_{X \times X,(x, x)}^{r+n . r}} .
$$

Then, we get two equations:

$$
\begin{gathered}
\sum_{l=1}^{k} \alpha_{l}\left(v_{l} \circ \pi_{1}\right)+\sum_{l=1}^{k} \beta_{l}\left(v_{l} \circ \pi_{2}\right)=0_{\mathscr{O}_{X \times X,(x, x)}^{r}} \\
\sum_{l=1}^{k} \beta_{l}\left(\left(z_{1} \circ \pi_{1}-z_{1} \circ \pi_{2}\right)\left(v_{l} \circ \pi_{2}\right), \ldots,\left(z_{n} \circ \pi_{1}-z_{n} \circ \pi_{2}\right)\left(v_{l} \circ \pi_{2}\right)\right)=0_{\mathscr{O}_{X \times X,(x, x)}^{n . r}}
\end{gathered}
$$

The equality (2) implies that

$$
\left(z_{i} \circ \pi_{1}-z_{i} \circ \pi_{2}\right) \sum_{l=1}^{k} \beta_{l}\left(v_{l} \circ \pi_{2}\right)=0_{\mathscr{O}_{X \times X,(x, x)}^{r}}
$$

for all $i \in\{1, \ldots, n\}$. Since $d \geq 1$ then $X$ is not a point. If $z_{i} \circ \pi_{1}-z_{i} \circ \pi_{2}=0_{\mathscr{O}_{X \times X,(x, x)}}$ for all $i$ then $X \times X \subseteq V(I(\Delta))=\Delta$ which implies $X \times X=\Delta$, so $X$ is a point, contradiction. So $z_{i} \circ \pi_{1}-$ $z_{i} \circ \pi_{2} \neq 0_{\mathscr{O}_{X \times X,(x, x)}}$, for some $i$. Since $(X, x)$ is irreducible then $(X \times X,(x, x))$ is irreducible, so $\mathscr{O}_{X \times X,(x, x)}$ is a domain, and by the equation (3) we have

$$
\sum_{l=1}^{k} \beta_{l}\left(v_{l} \circ \pi_{2}\right)=0_{\mathscr{O}_{X \times X,(x, x)}^{r}} .
$$

We can find an open subset $U$ of $X$, with $x \in U$, such that all $v_{1}, \ldots, v_{k}$ are defined on $U$, and all $\beta_{1}, \ldots, \beta_{k}$ are defined on $U \times U$. For each $z \in U$ define

$$
\begin{aligned}
\beta_{l}^{z}: U & \longrightarrow \mathbb{C} \\
w & \longmapsto \beta_{l}(z, w)
\end{aligned}
$$

Then, the germ $\beta_{l}^{z} \in \mathscr{O}_{X, x}, \forall z \in U$ and $l \in\{1, \ldots, k\}$. 
Let us prove that $\beta_{l}^{z}=0_{\mathscr{O}_{X, x}}, \forall z \in U$ and $l \in\{1, \ldots, k\}$. In fact, given $z$ and $l$, by the equation (4), for all $w \in U$ we have

$$
0_{\mathbb{C}^{r}}=\left(\sum_{l=1}^{k} \beta_{l}\left(v_{l} \circ \pi_{2}\right)\right)(z, w)=\sum_{l=1}^{k} \beta_{l}(z, w) v_{l}(w)=\left(\sum_{l=1}^{k} \beta_{l}^{z} v_{l}\right)(w)
$$

which implies $\sum_{l=1}^{k} \beta_{l}^{z} v_{l}=0_{\mathscr{O}_{X, x}^{r}}$. Since $v_{1}, \ldots, v_{k}$ are $\mathscr{O}_{X, x}$-linearly independent then $\beta_{l}^{z}=0_{\mathscr{O}_{X, x}}$.

Hence, $\beta_{l}=0_{\mathscr{O}_{X \times X,(x, x)}}$, for all $l \in\{1, \ldots, k\}$. So, the equation (1) becomes

$$
\sum_{l=1}^{k} \alpha_{l}\left(v_{l} \circ \pi_{1}\right)=0_{\mathscr{O}_{X \times X,(x, x)}^{r}}
$$

which is similar to the equation (4). Using the same argument, we conclude that $\alpha_{l}=0_{\mathscr{O}_{X \times X,(x, x)}}$, for all $l \in\{1, \ldots, k\}$. Thus, the Claim 1 is proved.

Hence, the generic rank of $M_{D}$ is $\geq 2 k$.

Claim 2: It does not exist more than $\mathbf{k}$ rows $\mathscr{O}_{X \times X,(x, x)}$-linearly independent on the first $p$ rows of $\left[M_{D}\right]$.

In fact, suppose that there exist. So, we have at least $k+1$ rows linearly independent on the first $\mathrm{p}$ rows of $\left[M_{D}\right]$. Without loss of generality, we can assume that the first $k+1$ rows satisfy it. Let us prove that $\left\{v_{1}, \ldots, v_{k+1}\right\}$ is $\mathscr{O}_{X, x}$-linearly independent. Indeed, let $\alpha_{1}, \ldots, \alpha_{k+1} \in \mathscr{O}_{X, x}$ such that $\sum_{l=1}^{k+1} \alpha_{l} v_{l}=0_{\mathscr{O}_{X, x}^{r}}$. Take $\lambda_{l}:=\alpha_{l} \circ \pi_{1}, \forall l \in\{1, \ldots, k+1\}$. For all $(z, w)$ in a small enough neighborhood of $(x, x)$ we have

$$
\left(\sum_{l=1}^{k+1} \lambda_{l} w_{l}\right)(z, w)=\left(\left(\sum_{l=1}^{k+1} \alpha_{l} v_{l}\right)(z), 0_{\mathbb{C}^{n . r}}\right)=0_{\mathbb{C}^{r+n . r}}
$$

which implies that $\sum_{l=1}^{k+1} \lambda_{l} w_{l}=0_{\mathscr{O}_{X \times X,(x, x)}^{r+n . r}}$. Since $\left\{w_{l}\right\}_{l=1}^{k+1}$ is linearly independent then $\lambda_{l}=$ $0_{\mathscr{O}_{X \times X,(x, x)}}$, for all $l \in\{1, \ldots, k+1\}$. Thus, for all $z$ in a small neighborhood of $x$ we have $0=\lambda_{l}(z, z)=\alpha_{l} \circ \pi_{1}(z, z)=\alpha_{l}(z)$, hence $\alpha_{l}=0_{\mathscr{O}_{X, x}}, \forall l \in\{1, \ldots, k+1\}$. Then, $v_{1}, \ldots, v_{k+1}$ is linearly independent and the generic rank of $M$ is $\geq k+1$, contradiction. Therefore, the Claim 2 is proved.

\section{Claim 3: It does not exist more than $\mathbf{k}$ rows $\mathscr{O}_{X \times X,(x, x)}$-linearly independent on the last $\mathbf{p}$ rows of $\left[\mathrm{M}_{\mathbf{D}}\right]$.}

In fact, suppose that there exist. So, we have at least $k+1$ rows linearly independent on the last $\mathrm{p}$ rows of $\left[M_{D}\right]$. Without loss of generality, we can assume that $\bar{w}_{1}, \ldots, \bar{w}_{k+1}$ are linearly independent. Let us prove that $\left\{v_{1}, \ldots, v_{k+1}\right\}$ is $\mathscr{O}_{X, x}$-linearly independent. Indeed, let $\alpha_{1}, \ldots, \alpha_{k+1} \in \mathscr{O}_{X, x}$ such that $\sum_{l=1}^{k+1} \alpha_{l} v_{l}=0_{\mathscr{O}_{X, x}^{r}}$. Take $\lambda_{l}:=\alpha_{l} \circ \pi_{2}, \forall l \in\{1, \ldots, k+1\}$. For all $(z, w)$ in a small enough neighborhood of $(x, x)$ we have

$$
\left(\sum_{l=1}^{k+1} \lambda_{l} \bar{w}_{l}\right)(z, w)=\left(\left(\sum_{l=1}^{k+1} \alpha_{l} v_{l}\right)(w),\left(z_{1}-w_{1}\right)\left(\left(\sum_{l=1}^{k+1} \alpha_{l} v_{l}\right)(w)\right), \ldots,\left(z_{n}-w_{n}\right)\left(\left(\sum_{l=1}^{k+1} \alpha_{l} v_{l}\right)(w)\right)\right)=0_{\mathbb{C}^{r+n . r}} .
$$


Since $\left\{\bar{w}_{l}\right\}_{l=1}^{k+1}$ is linearly independent then $\lambda_{l}=0_{\mathscr{O}_{X \times X}(x, x)}$, for all $l \in\{1, \ldots, k+1\}$, and $\alpha_{l}=0_{\mathscr{O}_{X, x}}$, $\forall l \in\{1, \ldots, k+1\}$. Then, $v_{1}, \ldots, v_{k+1}$ is linearly independent and the generic rank of $M$ is $\geq k+1$, contradiction. Therefore, the Claim 3 is proved.

Finally, suppose by contradiction that the generic rank of $M_{D}$ is $\geq 2 k+1$. Then we have $2 k+1$ rows of $\left[M_{D}\right]$ which are $\mathscr{O}_{X \times X,(x, x)}$-linearly independent. By the Claim 2, we have at most $k$ of these rows on the first $\mathrm{p}$ rows of $\left[M_{D}\right]$. Then, we have at least $k+1$ of these rows on the last p rows, which contradicts the Claim 3 . Therefore, the generic rank of $M_{D}$ is $2 k$.

The proof for the family case is completely analogous working on the fibered product $X \underset{Y}{\times} X$

Corollary 2.1.18. Let $\left\{V_{i}\right\}$ be the irreducible components of $(X, x)$. For each $i$, if $M$ has generic rank $k_{i}$ on $V_{i}$ then $M_{D}$ has generic rank $2 k_{i}$ on $V_{i} \times V_{i}$.

Remark 2.1.19. Let $M$ be an $\mathscr{O}_{X, x}$-submodule of $\mathscr{O}_{X, x}^{p}$ of generic rank $k$, and suppose that $\operatorname{dim} X=0$. Then $X$ is a point and $\mathscr{O}_{X, x} \cong \mathbb{C}$. In this case, $M$ is a $k$-dimensional $\mathbb{C}$-vector subspace of $\mathbb{C}^{p}$. Notice that, since $X \times X$ is also a point, then $\pi_{1}=\pi_{2}$. Hence, the map

$$
\begin{array}{ccc}
M \subseteq \mathbb{C}^{p} & \longrightarrow & M_{D} \subseteq \mathbb{C}^{2 p} \\
h & \longmapsto & (h, h)
\end{array}
$$

is an isomorphism of $\mathbb{C}$-vector spaces which implies that $\operatorname{dim}_{\mathbb{C}} M_{D}=k$, i.e, $M_{D}$ has generic rank $k$.

In next proposition we prove that the integral closure of modules commutes with finite direct sum of modules.

Proposition 2.1.20. Let $M \subseteq \mathscr{O}_{X, x}^{p}$ and $N \subseteq \mathscr{O}_{X, x}^{q}$ be $\mathscr{O}_{X, x}$-submodules. Then

$$
\overline{M \oplus N}=\bar{M} \oplus \bar{N} .
$$

Proof. First we prove that $\bar{M} \oplus 0 \subseteq \overline{M \oplus N}$. Let $v \in \bar{M} \oplus 0$. Then we can write $v=(g, 0)$ with $g \in \bar{M}$. Let $\phi:(\mathbb{C}, 0) \rightarrow(X, x)$ be an arbitrary analytic curve. Since $g \in \bar{M}$ then we can write $g \circ \phi=\sum_{i} \alpha_{i}\left(g_{i} \circ \phi\right)$, with $g_{i} \in M$ and $\alpha_{i} \in \mathscr{O}_{\mathbb{C}, 0}$, for all i. Thus, $v \circ \phi=(g \circ \phi, 0)$ $=\left(\sum_{i} \alpha_{i}\left(g_{i} \circ \phi\right), 0\right)=\sum_{i} \alpha_{i}\left(g_{i} \circ \phi, 0\right)=\sum_{i} \alpha_{i} \phi^{*}\left(g_{i}, 0\right) \in \phi^{*}(M \oplus N)$. Hence, $v \in \overline{M \oplus N}$ and $\bar{M} \oplus 0 \subseteq$ $\overline{M \oplus N}$. Analogously, $0 \oplus \bar{N} \subseteq \overline{M \oplus N}$. Therefore, $\bar{M} \oplus \bar{N} \subseteq \overline{M \oplus N}$.

Conversely, let $v \in \overline{M \oplus N}$. We can write $v=(g, h)$ with $g \in \mathscr{O}_{X, x}^{p}$ and $h \in \mathscr{O}_{X, x}^{q}$. Let $\phi:(\mathbb{C}, 0) \rightarrow(X, x)$ be an arbitrary analytic curve. Then we can write $\phi^{*}(v)=\sum_{i} \alpha_{i} \phi^{*}\left(g_{i}, h_{i}\right)$ where $g_{i} \in M, h_{i} \in N$ and $\alpha_{i} \in \mathscr{O}_{\mathbb{C}, 0}$, for all $i$. Comparing the coordinates of the last equation we conclude that $\phi^{*}(g)=\sum_{i} \alpha_{i} \phi^{*}\left(g_{i}\right)$ and $\phi^{*}(h)=\sum_{i} \alpha_{i} \phi^{*}\left(h_{i}\right)$. Thus, $g \in \bar{M}$ and $h \in \bar{N}$. Therefore, $v \in \bar{M} \oplus \bar{N}$. 
Corollary 2.1.21. Let $M_{i} \subseteq \mathscr{O}_{X, x}^{p_{i}}$ be $\mathscr{O}_{X, x}$-submodules, $i \in\{1, \ldots, r\}$. Then

$$
\overline{\bigoplus_{i=1}^{r} M_{i}}=\bigoplus_{i=1}^{r} \overline{M_{i}} .
$$

Proof. Induction on $r$ and apply the Proposition 2.1.20.

Let us recall some basic results in commutative algebra.

Remark 2.1.22. Let $(R, m)$ be a local ring and consider the residue field $k:=\frac{R}{m}$.

a) If $M$ is a free R-module of rank $p$ then $\frac{M}{m M}$ is a $k$-vector space of dimension $p$;

b) If $M$ is a free $R$-submodule of $R^{p}$ of rank $p$ then $M=R^{p}$.

Proof. (a) By hypothesis there exists an $R$-basis $\left\{v_{1}, \ldots v_{p}\right\}$ of $M$. Clearly the images of these elements in $\frac{M}{m M}$ form a generator set of $\frac{M}{m M}$. So, it remains to prove that these elements are $R$-linearly independent. Suppose we have an equation

$$
\sum_{i=1}^{p}\left(\alpha_{i}+m\right)\left(v_{i}+m M\right)=0+m M
$$

with $\alpha_{i} \in R$, for all $i \in\{1, \ldots, p\}$. Then $\sum_{i=1}^{p} \alpha_{i} v_{i} \in m M$ which implies that we can write $\sum_{i=1}^{p} \alpha_{i} v_{i}=$ $\sum_{i=1}^{p} \beta_{i} v_{i}$, for some $\beta_{1}, \ldots, \beta_{p} \in m$. Since $\left\{v_{1}, \ldots, v_{p}\right\}$ is $R$-linearly independent then $\alpha_{i}=\beta_{i} \in m$, for all $i \in\{1, \ldots, p\}$. Hence, $\alpha_{i}+m=0+m$, for all $i \in\{1, \ldots, p\}$.

(b) By hypothesis the submodule $M$ has an $R$-basis $\left\{v_{1}, \ldots, v_{p}\right\}$. Thus, $\left\{v_{1}, \ldots, v_{p}\right\}$ is an $R$-linearly independent subset of $R^{p}$. By the proof of the item (a) we have that $\left\{v_{1}+m R^{p}, \ldots, v_{p}+m R^{p}\right\}$ is a $k$-linearly independent subset of $\frac{R^{p}}{m R^{p}}$. By the item (a) we have $\operatorname{dim}_{k} \frac{R^{p}}{m R^{p}}=p$, hence $\left\{v_{1}+m R^{p}, \ldots, v_{p}+m R^{p}\right\}$ is a $k$-basis of $\frac{R^{p}}{m R^{p}}$. By Nakayama's Lemma $\left\{v_{1}, \ldots, v_{p}\right\}$ is a generator set of $R^{p}$ and we conclude that $M=R^{p}$.

Remark 2.1.23. Let $M$ be an $\mathscr{O}_{X, x}$-submodule of $\mathscr{O}_{X, x}^{p}$. If $\bar{M}=\mathscr{O}_{X, x}^{p}$ then $M=\mathscr{O}_{X, x}^{p}$.

In fact, let $s$ be the generic rank of $M$. Take an analytic curve $\phi:(\mathbb{C}, 0) \rightarrow(X, x)$ such that the rank of $\phi^{*}(M)$ is generically s. Since $\bar{M}=\mathscr{O}_{X, x}^{p}$ then $\phi^{*}(M)=\phi^{*}\left(\mathscr{O}_{X, x}^{p}\right)$ which has rank $p$, hence $s=p$. Thus, $M$ has generic rank $p$ and there exists a free $\mathscr{O}_{X, x}$-submodule $M_{p}$ of $M$ of rank p. By Remark 2.1.22 (b) we have that $M_{p}=\mathscr{O}_{X, x}^{p}$. Since $M_{p} \subseteq M$ then $M=\mathscr{O}_{X, x}^{p}$.

Therefore, in order to verify the equation $M=\mathscr{O}_{X, x}^{p}$ it suffices to check that $\bar{M}=\mathscr{O}_{X, x}^{p}$, which may be easier sometimes.

In the next proposition we calculate the stalk of the double sheaf $\mathscr{M}_{D}$ of a sheaf of modules $\mathscr{M}$ in a pair of points of $X$ such that one of them is not a singular point of $\mathscr{M}$, i.e, the matrix $[\mathscr{M}]$ in one of these points has maximal rank $p$. 
Proposition 2.1.24. Let $\mathscr{M} \subseteq \mathscr{O}_{X}^{p}$ be a sheaf of submodules. Let $\left(x, x^{\prime}\right) \in X \times X$ with $x \neq x^{\prime}$.

a) If $\mathscr{M}_{x}=\mathscr{O}_{X, x}^{p}$ then $\overline{\mathscr{M}_{D}}=\mathscr{O}_{X \times X,\left(x, x^{\prime}\right)}^{p} \oplus \overline{\left(\overline{\mathscr{M}_{x^{\prime}}} \circ \pi_{2}\right)}$ at $\left(x, x^{\prime}\right)$;

b) If $\mathscr{M}_{x^{\prime}}=\mathscr{O}_{X, x^{\prime}}^{p}$ then $\overline{\mathscr{M}_{D}}=\overline{\left(\overline{\mathscr{M}_{x}} \circ \pi_{1}\right)} \oplus \mathscr{O}_{X \times X,\left(x, x^{\prime}\right)}^{p}$ at $\left(x, x^{\prime}\right)$.

The same result still holds in the family case, working on the fibered product $X \underset{Y}{\times}$.

Proof. We may assume that $\mathscr{M}$ is generated by global sections $\left\{g_{1}, \ldots, g_{r}\right\}$. Since $x \neq x^{\prime}$ then $\left(z_{l} \circ \pi_{1}-z_{l} \circ \pi_{2}\right)\left(x, x^{\prime}\right) \neq 0$, for some $l \in\{1, \ldots, n\}$, thus $z_{l} \circ \pi_{1}-z_{l} \circ \pi_{2}$ is an invertible element of $\mathscr{O}_{X \times X,\left(x, x^{\prime}\right)}$.

(a) Let us prove that $\left(0, h \circ \pi_{2}\right) \in \overline{\mathscr{M}_{D}}$ at $\left(x, x^{\prime}\right), \forall h \in \overline{\mathscr{M}_{x^{\prime}}}$. In fact, let $\phi:(\mathbb{C}, 0) \rightarrow$ $\left(X \times X,\left(x, x^{\prime}\right)\right)$ be an arbitrary analytic curve. Write $\phi=\left(\phi_{1}, \phi_{2}\right)$. Since $h \in \overline{\mathscr{M}_{x^{\prime}}}$ then we can write $h \circ \phi_{2}=\sum_{i} \alpha_{i}\left(g_{i} \circ \phi_{2}\right)$ with $\alpha_{i} \in \mathscr{O}_{\mathbb{C}, 0}$, for all $i$. Since $\left(0,\left(z_{l} \circ \pi_{1}-z_{l} \circ \pi_{2}\right)\left(g_{i} \circ \pi_{2}\right)\right) \in \mathscr{M}_{D}$ at $\left(x, x^{\prime}\right)$, for all $i$, and $z_{l} \circ \pi_{1}-z_{l} \circ \pi_{2}$ is invertible then $\left(0, g_{i} \circ \pi_{2}\right) \in \mathscr{M}_{D}$ at $\left(x, x^{\prime}\right)$, for all $i$. Hence, $\left(0, h \circ \pi_{2}\right) \circ \phi=\left(0, h \circ \phi_{2}\right)=\sum_{i} \alpha_{i}\left(0, g_{i} \circ \phi_{2}\right)=\sum_{i} \alpha_{i}\left(\left(0, g_{i} \circ \pi_{2}\right) \circ \phi\right) \in \phi^{*}\left(\mathscr{M}_{D}\right)$. Therefore, $\left(0, h \circ \pi_{2}\right) \in \overline{\mathscr{M}}_{D}$ at $\left(x, x^{\prime}\right)$ and we conclude that $0 \oplus\left(\frac{i}{\mathscr{M}_{x^{\prime}}} \circ \pi_{2}\right) \subseteq \overline{\mathscr{M}_{D}}$ at $\left(x, x^{\prime}\right)$.

Let $e_{1}, \ldots, e_{p}$ be the canonical basis elements of $\mathscr{O}_{X}^{p}$. Since all these elements belong to $\mathscr{M}$ at $x$ then $\left(\left(z_{l} \circ \pi_{1}-z_{l} \circ \pi_{2}\right)\left(e_{j} \circ \pi_{1}\right), 0\right) \in \mathscr{M}_{D}$ at $\left(x, x^{\prime}\right), \forall j \in\{1, \ldots, p\}$. The element $z_{l} \circ \pi_{1}-z_{l} \circ \pi_{2}$ is invertible, hence $\left(e_{j} \circ \pi_{1}, 0\right) \in \mathscr{M}_{D}$ at $\left(x, x^{\prime}\right), \forall j \in\{1, \ldots, p\}$. Thus, $\mathscr{O}_{X \times X,\left(x, x^{\prime}\right)}^{p} \oplus 0 \subseteq \mathscr{M}_{D} \subseteq \overline{\mathscr{M}_{D}}$ at $\left(x, x^{\prime}\right)$, and $\mathscr{O}_{X \times X,\left(x, x^{\prime}\right)}^{p} \oplus\left(\overline{\mathscr{M}_{x^{\prime}}} \circ \pi_{2}\right) \subseteq \overline{\mathscr{M}_{D}}$ at $\left(x, x^{\prime}\right)$.

Clearly $\mathscr{M}_{D} \subseteq \mathscr{O}_{X \times X,\left(x, x^{\prime}\right)}^{p} \oplus\left(\mathscr{M}_{x^{\prime}} \circ \pi_{2}\right) \subseteq \mathscr{O}_{X \times X,\left(x, x^{\prime}\right)}^{p} \oplus\left(\overline{\mathscr{M}_{x^{\prime}}} \circ \pi_{2}\right)$ at $\left(x, x^{\prime}\right)$. Therefore,

$$
\mathscr{M}_{D} \subseteq \mathscr{O}_{X \times X,\left(x, x^{\prime}\right)}^{p} \oplus\left(\overline{\mathscr{M}_{x^{\prime}}} \circ \pi_{2}\right) \subseteq \overline{\mathscr{M}_{D}}
$$

at $\left(x, x^{\prime}\right)$ and using the Proposition 2.1.20 we conclude that $\overline{\mathscr{M}_{D}}=\overline{\mathscr{O}_{X \times X,\left(x, x^{\prime}\right)}^{p} \oplus\left(\overline{\mathscr{M}_{x^{\prime}}} \circ \pi_{2}\right)}=$ $\overline{\mathscr{O}_{X \times X,\left(x, x^{\prime}\right)}^{p}} \oplus \overline{\left(\overline{\mathscr{M}_{x^{\prime}}} \circ \pi_{2}\right)}=\mathscr{O}_{X \times X,\left(x, x^{\prime}\right)}^{p} \oplus \overline{\left(\overline{\mathscr{M}_{x^{\prime}}} \circ \pi_{2}\right)}$ at $\left(x, x^{\prime}\right)$.

(b) It is quite analogous to the item (a).

Corollary 2.1.25. Let $\mathscr{M} \subseteq \mathscr{O}_{X}^{p}$ be a sheaf of submodules. Let $\left(x, x^{\prime}\right) \in X \times X$ with $x \neq x^{\prime}$. If $\mathscr{M}_{x}=\mathscr{O}_{X, x}^{p}$ or $\mathscr{M}_{x^{\prime}}=\mathscr{O}_{X, x^{\prime}}^{p}$ then

$$
\overline{(\overline{\mathscr{M}})_{D}}=\overline{\mathscr{M}_{D}} \text { at }\left(x, x^{\prime}\right)
$$

The same result still holds in the family case, working on the fibered product $X \underset{Y}{X}$.

Proof. Assume that $\mathscr{M}_{x}=\mathscr{O}_{X, x}^{p}$ (the another case is analogous). By Proposition 2.1.24 (a) we have that

$$
\overline{\mathscr{M}_{D}}=\mathscr{O}_{X \times X,\left(x, x^{\prime}\right)}^{p} \oplus \overline{\left(\overline{\mathscr{M}_{x^{\prime}}} \circ \pi_{2}\right)}
$$


at $\left(x, x^{\prime}\right)$. Since $\overline{\mathscr{M}}_{x}=\mathscr{O}_{X, x}^{p}$ then applying again the Proposition 2.1.24 (a) we get

$$
\overline{(\overline{\mathscr{M}})_{D}}=\mathscr{O}_{X \times X,\left(x, x^{\prime}\right)}^{p} \oplus \overline{\left.\overline{\left(\overline{\mathscr{M}_{x^{\prime}}}\right.} \circ \pi_{2}\right)}
$$

at $\left(x, x^{\prime}\right)$. We already know that $\overline{\overline{\mathscr{M}_{x^{\prime}}}}=\overline{\mathscr{M}_{x^{\prime}}}$. Therefore $\overline{\overline{(\mathscr{M}})_{D}}=\overline{\mathscr{M}_{D}}$ at $\left(x, x^{\prime}\right)$.

Now we calculate the stalk of the double sheaf $\mathscr{M}_{D}$ at a point of the diagonal of $X$ whose corresponding point in $X$ is a non-singular point of $\mathscr{M}$.

Proposition 2.1.26. Let $\mathscr{M} \subseteq \mathscr{O}_{X}^{p}$ be a sheaf of submodules, $x \in X$ such that $\mathscr{M}_{x}=\mathscr{O}_{X, x}^{p}$. Then:

a) $\mathscr{M}_{D}=I_{\Delta}^{\oplus p} \oplus I_{\Delta}^{\oplus p}+E$ at $(x, x)$, where $E$ is the submodule generated by

$\left\{\left(e_{i}\right)_{D} \mid i \in\{1, \ldots, p\}\right\}$, where $e_{1}, \ldots, e_{p}$ are the canonical basis elements of $\mathscr{O}_{X}^{p}$;

b) $\overline{(\overline{\mathscr{M}})_{D}}=\overline{\mathscr{M}_{D}}$ at $(x, x)$.

The same result still holds in the family case, working on the fibered product $\underset{Y}{\underset{Y}{X}}$.

Proof. For each $i \in\{1, \ldots, p\}$ we have that $e_{i} \in \mathscr{M}_{x}$, which implies that

$\left(\left(z_{j} \circ \pi_{1}-z_{j} \circ \pi_{2}\right)\left(e_{i} \circ \pi_{1}\right), 0\right),\left(0,\left(z_{j} \circ \pi_{1}-z_{j} \circ \pi_{2}\right)\left(e_{i} \circ \pi_{2}\right)\right) \in \mathscr{M}_{D}$ at $(x, x)$, for all $j \in\{1, \ldots, n\}$ and $i \in\{1, \ldots, p\}$. Hence, $I_{\Delta}^{\oplus p} \oplus I_{\Delta}^{\oplus p} \subseteq \mathscr{M}_{D}$ at $(x, x)$. Since $E \subseteq \mathscr{M}_{D}$ at $(x, x)$ then $I_{\Delta}^{\oplus p} \oplus I_{\Delta}^{\oplus p}+E \subseteq$ $\mathscr{M}_{D}$ at $(x, x)$.

Notice that $\left(0,\left(z_{j} \circ \pi_{1}-z_{j} \circ \pi_{2}\right)\left(e_{i} \circ \pi_{2}\right)\right) \in I_{\Delta}^{\oplus p} \oplus I_{\Delta}^{\oplus p}$, for all $j \in\{1, \ldots, n\}$ and $i \in$ $\{1, \ldots, p\}$. Thus, all the generators of $\mathscr{M}_{D}$ at $(x, x)$ belong to $I_{\Delta}^{\oplus p} \oplus I_{\Delta}^{\oplus p}+E$, hence $\mathscr{M}_{D} \subseteq$ $I_{\Delta}^{\oplus p} \oplus I_{\Delta}^{\oplus p}+E$ at $(x, x)$. Therefore,

$$
\mathscr{M}_{D}=I_{\Delta}^{\oplus p} \oplus I_{\Delta}^{\oplus p}+E
$$

at $(x, x)$.

(b) Since $\mathscr{M}_{x}=\mathscr{O}_{X, x}^{p}$ then $\overline{\mathscr{M}}_{x}=\mathscr{M}_{x}$ at $x$, which implies that $(\overline{\mathscr{M}})_{D}=\mathscr{M}_{D}$ at $(x, x)$, and taking the integral closure we conclude that $\overline{(\overline{\mathscr{M}})_{D}}=\overline{\mathscr{M}_{D}}$ at $(x, x)$.

We use the previous results to obtain an algebraic invariant associated to an analytic variety with isolated singularity.

Let $F:\left(\mathbb{C}^{n}, 0\right) \rightarrow\left(\mathbb{C}^{p}, 0\right)$ be an analytic map, $n \geq p$ such that $X=F^{-1}(0)$ has an isolated singularity at the origin, $d=\operatorname{dim} X$ and $J M(F)$ the jacobian module defined on $X$. In general the submodule $(J M(F))_{D}$ does not have finite colength in $\mathscr{O}_{X \times X}^{2 p}$, even if $X$ is an isolated hypersurface singularity. Nevertheless, the multiplicity of the pair offers a way around this. The module $(J M(F))_{D}$ has a simple description, as we will see, off the origin in its cosupport, and any integral closure condition we wish to use is easily checked because of this structure. In the notation of [43], let $H_{2 d-1}\left((J M(F))_{D}\right)$ be the largest sheaf of modules whose integral closure 
agrees with $(J M(F))_{D}$ off the origin. This is the integral hull of $(J M(F))_{D}$ of codimension $2 d-1$, which means the integral closure of $(J M(F))_{D}$ and $H_{2 d-1}\left((J M(F))_{D}\right)$ agree off a set of codimension $2 d$, i.e, off $(0,0)$ in $X^{d} \times X^{d}$.

The next result identifies $H_{2 d-1}\left((J M(F))_{D}\right)$ and is a generalization of the Lemma 3.3 of [39].

Corollary 2.1.27. Let $F:\left(\mathbb{C}^{n}, 0\right) \rightarrow\left(\mathbb{C}^{p}, 0\right)$ be an analytic map with $n \geq p$ and suppose that $X=F^{-1}(0)$ has isolated singularity at the origin. Let $J M(F)$ be the jacobian module defined in $X$. Then the multiplicity of the pair of modules $e\left((J M(F))_{D},(\overline{J M(F)})_{D}\right)$ is well defined at the origin and if $X$ is an ICIS then

$$
H_{2 d-1}\left((J M(F))_{D}\right)=(\overline{J M(F)})_{D}
$$

Proof. By Corollary 2.1.25 and Proposition 2.1.26 (b) we have that $\overline{(\overline{J M(F)})_{D}}=\overline{(J M(F))_{D}}$ off the origin.

As a corollary of the proof of the last result, we have that

$$
H_{2 d-1}\left(\mathscr{M}_{D}\right)=(\overline{\mathscr{M}})_{D}
$$

for any sheaf of submodules $\mathscr{M} \subseteq \mathscr{O}_{X}^{p}$ of finite colength in $\mathscr{O}_{X}^{p}$.

\subsection{The infinitesimal Lipschitz conditions $i L_{A}$ and $i L_{m_{Y}}$}

Now we use some of the results presented in last section to recover some properties about the infinitesimal Lipschitz conditions for the following more general setup.

Setup 2.2.1. Let $(X, 0) \subseteq\left(\mathbb{C}^{n+k}, 0\right)$ be the germ of the analytic space defined by an analytic map $F: \mathbb{C}^{n} \times \mathbb{C}^{k} \rightarrow \mathbb{C}^{p}$, where $X$ is a sufficient small representative such that $Y=\mathbb{C}^{k}=0 \times \mathbb{C}^{k} \subseteq X$ is the singular set of $X$. Let $F_{1}, \ldots, F_{p}: \mathbb{C}^{n} \times \mathbb{C}^{k} \rightarrow \mathbb{C}$ be the coordinates functions of $F$, for each $y \in Y$ let $f_{y}: \mathbb{C}^{n} \rightarrow \mathbb{C}^{p}$ given by $f_{y}(z):=F(z, y)$ and let $X_{y}:=f_{y}^{-1}(0)$. Assume that $X_{y}$ has an isolated singularity in 0 , for all $y \in Y$ and $n \geq p$. Let $z_{1}, \ldots, z_{n}, y_{1}, \ldots, y_{k}$ be the coordinates on $\mathbb{C}^{n+k}$, let $m_{Y}$ be the ideal of $\mathscr{O}_{X}$ generated by $\left\{z_{1}, \ldots, z_{n}\right\}$, let $J M(X)$ be the jacobian module of $X$, let $J M(X)_{Y}$ be the module generated by $\left\{\frac{\partial F}{\partial y_{1}}, \ldots, \frac{\partial F}{\partial y_{k}}\right\}$ and let $J M_{z}(X)$ be the module generated by $\left\{\frac{\partial F}{\partial z_{1}}, \ldots, \frac{\partial F}{\partial z_{n}}\right\}$.

In this section we work with the doubles relative to $Y$ and with the projections

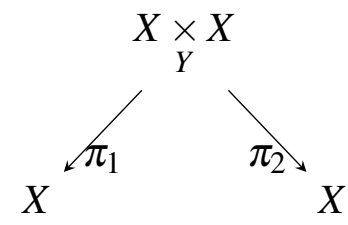


Since $X_{y}$ has an isolated singularity in 0 , for all $y \in Y$, then $\Sigma\left(J M_{z}(X)\right)=\{(0, y) \mid y \in$ $Y\}=Y$, so by Theorem 2.1.6 we have that

$$
\operatorname{cosupp}\left(\left(J M_{z}(X)\right)_{D}\right)=\Delta(X) \cup(\underset{Y}{\times} 0) \cup(\underset{Y}{\times} X) .
$$

Definition 2.2.2. Under the above setup, we introduce the infinitesimal Lipschitz conditions.

- The pair $(X, Y)$ satisfies the infinitesimal Lipschitz condition $\mathbf{m}_{\mathbf{Y}}\left(\mathbf{i L}_{\mathbf{m}_{\mathbf{Y}}}\right)$ at $\left(x, x^{\prime}\right) \in \underset{Y}{\underset{X}{X}}$ if $\left(J M(X)_{Y}\right)_{D} \subseteq \overline{\left(m_{Y} J M_{z}(X)\right)_{D}}$ at $\left(x, x^{\prime}\right)$;

- The pair $(X, Y)$ satisfies the infinitesimal Lipschitz condition $A\left(\mathbf{i L}_{\mathbf{A}}\right)$ at $\left(x, x^{\prime}\right) \in \underset{Y}{\times} \underset{X}{X}$ if $\left(J M(X)_{Y}\right)_{D} \subseteq \overline{\left(J M_{Z}(X)\right)_{D}}$ at $\left(x, x^{\prime}\right)$.

Notice that $i L_{m_{Y}}$ implies $i L_{A}$.

By Theorem 2.5 of [41], the pair $(X, Y)$ satisfies the Verdier $(w)$-regularity at $x \in X$ if and only if $J M(X)_{Y} \subseteq \overline{m_{Y} J M_{Z}(X)}$ at $x$. Thus, the $i L_{m_{Y}}$ condition is the Lipschitz version of the $W$ condition. The $i L_{A}$ condition is the Lipschitz version of the $A_{F}$ condition, which is $J M(X)_{Y} \subseteq \overline{J M_{Z}(X)}$, if this holds on the ambient space.

Example 2.2.3. (Family of cusps) Consider $X \subseteq \mathbb{C}^{3}$ given by the equation $F(x, y, z)=z^{2}-x^{3}=0$. Since the gradient of $F$ at a point $(x, y, z)$ is $\left(-3 x^{2}, 2 z, 0\right)$ then the singular locus of $X$ is the $y$-axis. In this case, it is easy to see that $J M(X)_{Y}=\{0\}$ and therefore both infinitesimal Lipschitz conditions are trivially satisfied at any point of $\underset{Y}{X} X$.

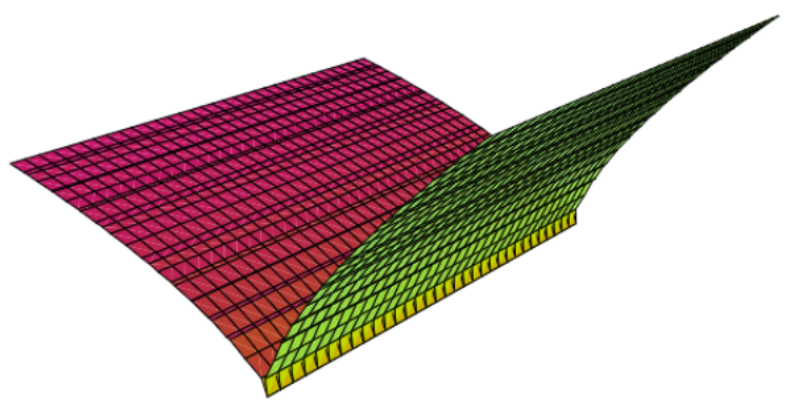

Figure 6 - Family of Cusps

In general, if $X$ is defined by an analytic map which does not depend of the parameter space then $J M(X)_{Y}=\{0\}$ and both infinitesimal Lipschitz conditions are trivially satisfied.

Example 2.2.4. Consider the analytic map $f(x, y)=x^{2}+y^{3}$. Taking $q:=\frac{3+1}{2}=2$ we have seen in Example 1.4.4 that $y^{2} \in \overline{J(f)}$ but $\left(y^{2}\right)_{D} \notin \overline{(J(f))_{D}}$. In this case, $J(f)_{Y}$ is generated by $y^{2}$. Then, $\left(J(f)_{Y}\right)_{D} \nsubseteq \overline{(J(f))_{D}}$.

Hence, neither of the infinitesimal Lipschitz conditions are satisfied. 
We want to see if the condition $i L_{m_{Y}}$ at a point of $(0,0) \times Y$ depends of the projection $\mathbf{p}: X \rightarrow Y$. The next lemma will be useful to answer this question.

Lemma 2.2.5. Consider the setup 2.2.1. For any parameter $y \in Y$ we have:

a) If $\left(J M(X)_{Y}\right)_{D} \subseteq \overline{\left(m_{Y} J M(X)\right)_{D}}$ at $(0, y, 0, y) \in(0,0) \times Y$ then

$$
J M(X)_{Y} \subseteq \overline{m_{Y} J M_{Z}(X)}
$$

at $(0, y)$, i.e, the $W$ condition holds at $(0, y)$;

b) If $\left(J M(X)_{Y}\right)_{D} \subseteq\left(\left(m_{Y} J M(X)\right)_{D}\right)^{\dagger}$ at $(z, y, z, y) \in \underset{Y}{X} \underset{Y}{\times}$ then

$$
J M(X)_{Y} \subseteq\left(m_{Y} J M_{Z}(X)\right)^{\dagger}
$$

at $(z, y)$.

Proof. (a) Let $\phi:(\mathbb{C}, 0) \rightarrow(X,(0, y))$ be an arbitrary analytic curve. By the hypothesis and Corollary 2.1.15 (a) we have that $J M(X)_{Y} \subseteq \overline{m_{Y} J M(X)}$, hence $\phi^{*}\left(J M(X)_{Y}\right) \subseteq \phi^{*}\left(m_{Y} J M(X)\right)$.

Now, since $\phi^{*}\left(m_{Y}\right) \subseteq m_{1}, J M(X)=J M(X)_{Y}+J M_{z}(X)$ and $\phi^{*}$ preserves the module operations then:

$\left.\phi^{*}\left(J M(X)_{Y}\right) \subseteq \phi^{*}\left(m_{Y} J M(X)\right)\right)=\phi^{*}\left(m_{Y} J M(X)_{Y}+m_{Y} J M_{z}(X)\right)$

$\subseteq m_{1} \phi^{*}\left(J M(X)_{Y}\right)+\phi^{*}\left(m_{Y} J M_{Z}(X)\right)$. By Nakayama's Lemma we conclude that $\phi^{*}\left(J M(X)_{Y}\right) \subseteq$ $\left.\phi^{*}\left(m_{Y} J M_{Z}(X)\right)\right)$. Therefore, (a) is proved by the curve criterion.

(b) Let $\phi:(\mathbb{C}, 0) \rightarrow(X,(z, y))$ be an arbitrary analytic curve. By the hypothesis and Corollary 2.1.15 (b) we have that $J M(X)_{Y} \subseteq\left(m_{Y} J M(X)\right)^{\dagger}$, hence $\phi^{*}\left(J M(X)_{Y}\right) \subseteq m_{1} \phi^{*}\left(m_{Y} J M(X)\right)$, and furthermore, $\phi^{*}\left(J M(X)_{Y}\right) \subseteq m_{1} \phi^{*}\left(m_{Y} J M(X)\right)=m_{1} \phi^{*}\left(m_{Y} J M(X)_{Y}\right)+m_{1} \phi^{*}\left(m_{Y} J M_{Z}(X)\right)$ $\subseteq m_{1} \phi^{*}\left(J M(X)_{Y}\right)+\left(m_{1} \phi^{*}\left(m_{Y} J M_{z}(X)\right)\right)$. By Nakayama's Lemma we have that $\phi^{*}\left(J M(X)_{Y}\right) \subseteq$ $m_{1} \phi^{*}\left(m_{Y} J M_{z}(X)\right)$. Therefore, (b) is proved.

The next result gives us a kind of "independence of the projection onto $Y$ " of the $i L_{m_{Y}}$ condition at the origin .

Proposition 2.2.6. Consider the setup 2.2.1. At any point of $(0,0) \times Y$, we have that $\left(J M(X)_{Y}\right)_{D} \subseteq$ $\overline{\left(m_{Y} J M(X)\right)_{D}}$ if and only if $\left(J M(X)_{Y}\right)_{D} \subseteq \overline{\left(m_{Y} J M_{Z}(X)\right)_{D}}$.

Proof. The implication $(\Longleftarrow)$ is obvious. Let us to prove $(\Longrightarrow)$.

Let $\phi:(\mathbb{C}, 0) \rightarrow(\underset{Y}{X} X,(0, y, 0, y))$ be an arbitrary analytic curve. Write $\phi=\left(\phi_{1}, \phi_{2}\right)$, with $\phi_{1}, \phi_{2}:(\mathbb{C}, 0) \rightarrow(X,(0, y))$.

Claim 1: $\left(\left(z_{i} \circ \phi_{1}-z_{i} \circ \phi_{2}\right)\left(\frac{\partial F}{\partial y_{l}} \circ \phi_{1}\right), 0\right) \in \phi^{*}\left(\left(m_{Y} J M_{z}(X)\right)_{D}\right)$, for all $i \in\{1, \ldots, n\}$ and $l \in\{1, \ldots, k\}$. 
In fact, by Lemma 2.2.5 (a) we have $(J M(X))_{Y} \subseteq \overline{\left(m_{Y} J M_{z}(X)\right)}$, so $\frac{\partial F}{\partial y_{l}} \in \overline{\left(m_{Y} J M_{z}(X)\right)} \Longrightarrow \frac{\partial F}{\partial y_{l}} \circ \phi_{1} \in \phi_{1}^{*}\left(m_{Y} J M_{z}(X)\right)$ and we can write $\frac{\partial F}{\partial y_{l}} \circ \phi_{1}=\sum_{r, j} \beta_{r j}\left(\left(z_{r} \frac{\partial F}{\partial z_{j}}\right) \circ \phi_{1}\right)$, with $\beta_{r j} \in \mathscr{O}_{\mathbb{C}, 0}$. Then, $\left(\left(z_{i} \circ \phi_{1}-z_{i} \circ \phi_{2}\right)\left(\frac{\partial F}{\partial y_{l}} \circ \phi_{1}\right), 0\right)=\sum_{r, j} \beta_{r j}\left(\left(z_{i} \circ \phi_{1}-z_{i} \circ \phi_{2}\right)\left(\left(z_{r} \frac{\partial F}{\partial z_{j}}\right) \circ \phi_{1}\right), 0\right)$

$=\sum_{r, j} \beta_{r j} \underbrace{\phi^{*}\left(\left(\left(z_{i} \circ \pi_{1}-z_{i} \circ \pi_{2}\right)\left(\left(z_{r} \frac{\partial F}{\partial z_{j}}\right) \circ \pi_{1}\right), 0\right)\right)}_{\in \phi^{*}\left(\left(m_{Y} J M_{z}(X)\right)_{D}\right)} \in \phi^{*}\left(\left(m_{Y} J M_{z}(X)\right)_{D}\right)$. So the Claim 1 is proved.

Claim 2: $\phi^{*}\left(\left(m_{Y} J M(X)_{Y}\right)_{D}\right) \subseteq m_{1} \phi^{*}\left(\left(m_{Y} J M(X)\right)_{D}\right)+\phi^{*}\left(\left(m_{Y} J M_{Z}(X)\right)_{D}\right)$.

In fact, it is enough to look to the images of the generators of $\left(m_{Y} J M(X)_{Y}\right)_{D}$. For all $i, j \in\{1, \ldots, n\}$ and $l \in\{1, \ldots, k\}$ we have

$$
\phi^{*}\left(\left(z_{i} \frac{\partial F}{\partial y_{l}}\right)_{D}\right)=\left(z_{i} \circ \phi_{2}\right)\left(\frac{\partial F}{\partial y_{l}} \circ \phi_{1}, \frac{\partial F}{\partial y_{l}} \circ \phi_{2}\right)+\left(\left(z_{i} \circ \phi_{1}-z_{i} \circ \phi_{2}\right)\left(\frac{\partial F}{\partial y_{l}} \circ \phi_{1}\right), 0\right) .
$$

We have that $z_{i} \circ \phi_{2} \in m_{1}$. By hypothesis we have $\phi^{*}\left(\left(J M(X)_{Y}\right)_{D}\right) \subseteq \phi^{*}\left(\left(m_{Y} J M(X)\right)_{D}\right)$, so $\phi^{*}\left(\left(\frac{\partial F}{\partial y_{l}}\right)_{D}\right) \in \phi^{*}\left(\left(m_{Y} J M(X)\right)_{D}\right)$. Thus, the first term on the right hand side of the above sum is in $m_{1} \phi^{*}\left(\left(m_{Y} J M(X)\right)_{D}\right)$. The second term on the right hand side of the above sum is in $\phi^{*}\left(\left(m_{Y} J M_{z}(X)\right)_{D}\right)$ by Claim 1 .

Hence, $\phi^{*}\left(\left(z_{i} \frac{\partial F}{\partial y_{l}}\right)_{D}\right) \in m_{1} \phi^{*}\left(\left(m_{Y} J M(X)\right)_{D}\right)+\phi^{*}\left(\left(m_{Y} J M_{z}(X)\right)_{D}\right)$. Also, we have $\phi^{*}\left(\left(z_{i} \circ \pi_{1}-z_{i} \circ \pi_{2}\right)\left(\left(z_{j} \frac{\partial F}{\partial y_{l}}\right) \circ \pi_{1}\right), 0\right)=\left(z_{j} \circ \phi_{1}\right) \underbrace{\left.\left(z_{i} \circ \phi_{1}-z_{i} \circ \phi_{2}\right)\left(\frac{\partial F}{\partial y_{l}} \circ \phi_{1}\right), 0\right)}_{\in \phi^{*}\left(\left(m_{Y} J M_{z}(X)\right)_{D}\right)}$, so the Claim 2 is proved.

Thus, $\phi^{*}\left(\left(m_{Y} J M(X)\right)_{D}\right)=\phi^{*}\left(\left(m_{Y} J M(X)_{Y}\right)_{D}\right)+\phi^{*}\left(\left(m_{Y} J M_{Z}(X)\right)_{D}\right)$

$\subseteq\left(m_{1} \phi^{*}\left(\left(m_{Y} J M(X)\right)_{D}\right)+\phi^{*}\left(\left(m_{Y} J M_{Z}(X)\right)_{D}\right)\right)+\phi^{*}\left(\left(m_{Y} J M_{Z}(X)\right)_{D}\right)$

$=m_{1} \phi^{*}\left(\left(m_{Y} J M(X)\right)_{D}\right)+\phi^{*}\left(\left(m_{Y} J M_{z}(X)\right)_{D}\right)$. By Nakayama's Lemma we conclude that $\phi^{*}\left(\left(m_{Y} J M(X)\right)_{D}\right) \subseteq \phi^{*}\left(\left(m_{Y} J M_{Z}(X)\right)_{D}\right)$.

Since $\phi^{*}\left(\left(J M(X)_{Y}\right)_{D}\right) \subseteq \phi^{*}\left(\left(m_{Y} J M(X)\right)_{D}\right)$ then $\phi^{*}\left(\left(J M(X)_{Y}\right)_{D}\right) \subseteq \phi^{*}\left(\left(m_{Y} J M_{Z}(X)\right)_{D}\right)$. By the curve criterion we conclude that $\left(J M(X)_{Y}\right)_{D} \subseteq \overline{\left(m_{Y} J M_{Z}(X)\right)_{D}}$.

While a similar result for $i L_{A}$ does not make sense, if we work on the strict $i L_{A}$, then we get an analogous result, as we can see in the following proposition.

Proposition 2.2.7. Consider the setup 2.2.1. At any point $\left(x, x^{\prime}\right) \in \underset{Y}{X} \underset{X}{X}$ we have:

$$
\left(J M(X)_{Y}\right)_{D} \subseteq\left(J M(X)_{D}\right)^{\dagger} \text { if and only if }\left(J M(X)_{Y}\right)_{D} \subseteq\left(J M_{z}(X)_{D}\right)^{\dagger}
$$

Proof. The implication $(\Longleftarrow)$ is obvious. Now, suppose that $\left(J M(X)_{Y}\right)_{D} \subseteq\left(J M(X)_{D}\right)^{\dagger}$, and let $\phi:(\mathbb{C}, 0) \rightarrow\left(X \times \underset{Y}{\times},\left(x, x^{\prime}\right)\right)$ be an arbitrary curve. Then,

$\phi^{*}\left(\left(J M(X)_{Y}\right)_{D}\right) \subseteq m_{1} \phi^{*}\left(J M(X)_{D}\right)=m_{1} \phi^{*}\left(\left(J M(X)_{Y}\right)_{D}\right)+m_{1} \phi^{*}\left(\left(J M_{Z}(X)\right)_{D}\right)$. By Nakayama's Lemma we conclude that $\phi^{*}\left(\left(J M(X)_{Y}\right)_{D}\right) \subseteq m_{1} \phi^{*}\left(J M_{Z}(X)_{D}\right)$, which finishes the proof. 
Lemma 2.2.8. Consider the setup 2.2.1. If $x=(z, y) \in X$ with $z \neq 0$ then $m_{Y} J M_{z}(X)=J M_{z}(X)=$ $\mathscr{O}_{X}^{p}$ at $x$.

Proof. Since $z \neq 0$ then the jacobian matrix $\left[J\left(f_{y}\right)(z)\right]=\left[J M_{z}(X)(x)\right]$ has maximal rank $p$. Without loss of generality we can assume that the $p \times p$ submatrix formed by the first $p$ columns of $\left[J M_{z}(X)(x)\right]$ is invertible. Denote this matrix by $\left[J M_{z}(X)(x)\right]_{p}$. Then, there exists an open subset $U$ of $X$ containing $x$ such that $\left[J_{z}(X)(w)\right]_{p}$ is invertible, $\forall w \in U$. Let $e_{1}, \ldots, e_{p}$ be the canonical generators of $\mathscr{O}_{X}^{p}$.

Let us to prove that $e_{j} \in J M_{z}(X)$ at $x, \forall j \in\{1, \ldots, p\}$. In fact, given such $j$, consider the analytic map $\alpha: U \rightarrow \mathbb{C}^{p}$ given by $\alpha(w):=\left[J M_{z}(X)(w)\right]_{p}^{-1} \cdot e_{j}$, with coordinates function $\alpha_{1}, \ldots, \alpha_{p}$. So, we have $e_{j}=\sum_{i=1}^{p} \alpha_{i} \frac{\partial F}{\partial z_{i}}$ on $U$, hence $e_{j} \in J M_{z}(X)$ at $x$. Therefore, $J M_{z}(X)=\mathscr{O}_{X}^{p}$ at $x$.

Now, since $z \neq 0$ then $z_{k}(x) \neq 0$ for some $k$, so $z_{k}$ is invertible in $\mathscr{O}_{X, x}$. Since $\frac{\partial F}{\partial z_{i}}=$ $z_{k}^{-1}\left(z_{k} \frac{\partial F}{\partial z_{i}}\right)$, for all $i \in\{1, \ldots, n\}$ then $J M_{z}(X)=m_{Y} J M_{z}(X)$ at $x$.

The next result generalizes the Proposition 4.1 of [39].

Proposition 2.2.9. Consider the setup 2.2.1. Then

$$
\left.\operatorname{cosupp}\left(\left(m_{Y} J M_{z}(X)\right)_{D}\right)=\operatorname{cosupp}\left(\left(J M_{z}(X)\right)_{D}\right)=\Delta(X) \cup \underset{Y}{X} \underset{X}{\times}\right) \cup(\underset{Y}{\times} X) .
$$

Proof. Since $\left(m_{Y} J M_{Z}(X)\right)_{D} \subseteq\left(J M_{Z}(X)\right)_{D}$ at any point, then $\operatorname{cosupp}\left(J M_{z}(X)_{D}\right) \subseteq \operatorname{cosupp}\left(m_{Y} J M_{z}(X)\right)_{D}$. We already know that

$$
\operatorname{cosupp}\left(\left(J M_{z}(X)\right)_{D}\right)=\Delta(X) \cup(\underset{Y}{\times} 0) \cup(\underset{Y}{\times} X) .
$$

Let us to prove that

$$
X \underset{Y}{\times} X-\left(\operatorname{cosupp}\left(\left(J M_{Z}(X)\right)_{D}\right)\right) \subseteq \underset{Y}{X} \underset{X}{\times}-\left(\operatorname{cosupp}\left(\left(m_{Y} J M_{Z}(X)\right)_{D}\right)\right) .
$$

In fact, if $\left(x, x^{\prime}\right)$ is not in the cosupport of $\left(J M_{z}(X)\right)_{D}$ then $\left(J M_{z}(X)\right)_{D}=\mathscr{O}_{X \times X}^{2 p}$ at $\left(x, x^{\prime}\right)$, and we can write $x=(z, y)$ and $x^{\prime}=\left(z^{\prime}, y\right)$, with $z \neq 0, z^{\prime} \neq 0$ and $z \neq z^{\prime}$. So there exist $t, k, r \in\{1, \ldots, n\}$ such that $z_{t} \circ \pi_{1}, z_{k} \circ \pi_{2}$ and $z_{r} \circ \pi_{1}-z_{r} \circ \pi_{2}$ are invertibles in $\mathscr{O}_{X \times X} \underset{Y}{ },\left(x, x^{\prime}\right)$.

Notice that: for all $j \in\{1, \ldots, n\}$ we have:

$$
\begin{aligned}
& \left(\frac{\partial F}{\partial z_{j}}\right)_{D}=\left(z_{t} \circ \pi_{1}\right)^{-1} \underbrace{\left(\left(z_{t} \frac{\partial F}{\partial z_{j}}\right) \circ \pi_{1},\left(z_{t} \frac{\partial F}{\partial z_{j}}\right) \circ \pi_{2}\right)}_{\in\left(m_{Y} J M_{z}(X)\right)_{D}} \\
& -\left(z_{t} \circ \pi_{1}\right)^{-1}\left(z_{r} \circ \pi_{1}-z_{r} \circ \pi_{2}\right)^{-1} \underbrace{\left(0,\left(z_{r} \circ \pi_{1}-z_{r} \circ \pi_{2}\right)\left(\left(z_{t} \frac{\partial F}{\partial z_{j}}\right) \circ \pi_{2}\right)\right)}_{\in\left(m_{Y} J M_{z}(X)\right)_{D}} \\
& +\left(z_{k} \circ \pi_{2}\right)^{-1}\left(z_{r} \circ \pi_{1}-z_{r} \circ \pi_{2}\right)^{-1} \underbrace{\left(0,\left(z_{r} \circ \pi_{1}-z_{r} \circ \pi_{2}\right)\left(\left(z_{k} \frac{\partial F}{\partial z_{j}}\right) \circ \pi_{2}\right)\right)}_{\in\left(m_{Y} J M_{z}(X)\right)_{D}} \in\left(m_{Y} J M_{z}(X)\right)_{D} .
\end{aligned}
$$


Furthermore, for all $i, j \in\{1, \ldots, n\}$ we have $\left(0,\left(z_{i} \circ \pi_{1}-z_{i} \circ \pi_{2}\right)\left(\frac{\partial F}{\partial z_{j}} \circ \pi_{2}\right)\right)$ $=\left(z_{k} \circ \pi_{2}\right)^{-1}\left(0,\left(z_{i} \circ \pi_{1}-z_{i} \circ \pi_{2}\right)\left(\left(z_{k} \frac{\partial F}{\partial z_{j}}\right) \circ \pi_{2}\right)\right) \in\left(m_{Y} J M_{z}(X)\right)_{D}$.

Thus, we conclude that $\left(m_{Y} J M_{Z}(X)\right)_{D}=\left(J M_{z}(X)\right)_{D}=\mathscr{O}_{X \times X}^{2 p}$ at $\left(x, x^{\prime}\right)$ $\Rightarrow\left(x, x^{\prime}\right) \notin \operatorname{cosupp}\left(\left(m_{Y} J M_{z}(X)\right)_{D}\right)$.

Hence, $\operatorname{cosupp}\left(m_{Y} J M_{z}(X)\right)_{D} \subseteq \operatorname{cosupp}\left(J M_{z}(X)\right)_{D}$, which finishes the proof.

The next result generalizes the Proposition 4.2 of [39], and states that both infinitesimal Lipschitz conditions hold in any point of $\underset{Y}{\times} X$ off $(0,0) \times Y$.

Proposition 2.2.10. Consider the setup 2.2.1.

a) If $(x, x) \in \Delta(X)-((0,0) \times Y)$ then $\left(J M(X)_{Y}\right)_{D} \subseteq\left(J M_{Z}(X)\right)_{D}$ and $\left(J M(X)_{Y}\right)_{D} \subseteq\left(m_{Y} J M_{z}(X)\right)_{D}$ at $(x, x)$. In particular, $i L_{A}$ and $i L_{m_{Y}}$ hold at $(x, x)$;

b) If $\left(x, x^{\prime}\right) \in(\underset{Y}{\times} \underset{\times}{\times}) \cup(\underset{Y}{\times} X)-((0,0) \times Y)$ and the $W$ condition holds in any point $(0, y)$, $y \in Y$, then $i L_{A}$ and $i L_{m_{Y}}$ conditions hold at $\left(x, x^{\prime}\right)$.

Proof. (a) We can write $x=(z, y)$ with $z \neq 0$. By Lemma 2.2.8 we have that $J M(X)_{Y} \subseteq \mathscr{O}_{X}^{p}=$ $J M_{z}(X)=m_{Y} J M_{z}(X)$ at $x$, which implies that $\left(J M(X)_{Y}\right)_{D} \subseteq\left(J M_{z}(X)\right)_{D}$ and $\left(J M(X)_{Y}\right)_{D} \subseteq$ $\left(m_{Y} J M_{z}(X)\right)_{D}$ at $(x, x)$.

(b) We can assume that $\left(x, x^{\prime}\right) \in(X \times 0)-((0,0) \times Y)$ (the other case is completely analougous). So, we can write $x=(z, y)$ and $x^{\prime}=(0, y)$, with $z \neq 0$. So $z_{k}(x) \neq 0$ for some $k$, and follows that $\left(z_{k} \circ \pi_{1}-z_{k} \circ \pi_{2}\right)\left(x, x^{\prime}\right)=z_{k}(x)-0=z_{k}(x) \neq 0 \Rightarrow z_{k} \circ \pi_{1}$ and $z_{k} \circ \pi_{1}-z_{k} \circ \pi_{2}$ are invertibles in $\mathscr{O}_{X \times X} \underset{Y}{ }\left(x, x^{\prime}\right)$. Let $e_{1}, \ldots, e_{p}$ be the canonical generators of $\mathscr{O}_{X}^{p} \times_{Y}^{p}$ and $e_{1}^{\prime}, \ldots, e_{p}^{\prime}$ be the canonical generators of $\mathscr{O}_{X}^{p}$. Thus $e_{j}=e_{j}^{\prime} \circ \pi_{1}, \forall j \in\{1, \ldots, p\}$. Since $z \neq 0$ then by Lemma 2.2.8 we have $J M_{z}(X)=\mathscr{O}_{X}^{p}$ at $x$.

Let us to prove that $\left(e_{j}, 0\right) \in\left(m_{Y} J M_{z}(X)\right)_{D}, \forall j \in\{1, \ldots, p\}$ at $\left(x, x^{\prime}\right)$. In fact, given a such $j$, we have $e_{j}^{\prime} \in \mathscr{O}_{X}^{p}=J M_{z}(X)$ at $x$, so we can write $e_{j}^{\prime}=\sum_{i=1}^{n} \alpha_{i} \frac{\partial F}{\partial z_{i}} \Rightarrow\left(e_{j}, 0\right)=\sum_{i=1}^{n}\left(\alpha_{i} \circ \pi_{1}\right)\left(z_{k} \circ\right.$ $\left.\pi_{1}-z_{k} \circ \pi_{2}\right)^{-1}\left(z_{k} \circ \pi_{1}\right)^{-1} \underbrace{\left(\left(z_{k} \circ \pi_{1}-z_{k} \circ \pi_{2}\right)\left(z_{k} \frac{\partial F}{\partial z_{i}}\right) \circ \pi_{1}, 0\right)}_{\in\left(m_{Y} J M_{z}(X)\right)_{D}}$, hence $\left(e_{j}, 0\right) \in\left(m_{Y} J M_{z}(X)\right)_{D}$.

Let us to prove that $\left(0, \frac{\partial F}{\partial y_{l}} \circ \pi_{2}\right) \in \overline{\left.\left(m_{Y} J M_{z}(X)\right)_{D}\right)}$ at $\left(x, x^{\prime}\right), \forall l \in\{1, \ldots, k\}$. In fact, given

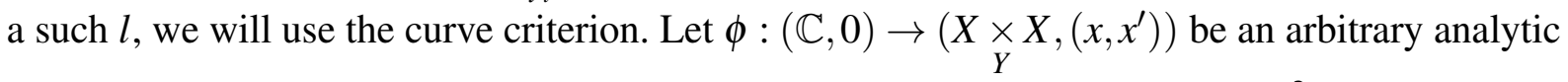
curve. Write $\phi=(\varphi, \gamma)$. Since $\gamma:(\mathbb{C}, 0) \rightarrow\left(X, x^{\prime}\right)$ is an analytic curve and $\frac{\partial F}{\partial y_{l}} \in \overline{m_{Y} J M_{z}(X)}$ at $x^{\prime}=(0, y)$ then $\frac{\partial F}{\partial y_{l}} \circ \gamma=g \circ \gamma$, for some $g \in m_{Y} J M_{z}(X)$ at $x^{\prime}$. So, we can write $g=\sum_{r, j=1}^{n} \beta_{r j} z_{r} \frac{\partial F}{\partial z_{j}}$. Thus, we have that $\left(0, g \circ \pi_{2}\right)=\sum\left(\beta_{r j} \circ \pi_{2}\right)\left(z_{k} \circ \pi_{1}-z_{k} \circ \pi_{2}\right)^{-1} \underbrace{\left(0,\left(z_{k} \circ \pi_{1}-z_{k} \circ \pi_{2}\right)\left(z_{r} \frac{\partial F}{\partial z_{j}}\right) \circ \pi_{2}\right)}_{\in\left(m_{Y} J M_{z}(X)\right)_{D}}$ 
$\Rightarrow\left(0, g \circ \pi_{2}\right) \in\left(m_{Y} J M_{z}(X)\right)_{D}$. Then, $\left(0, \frac{\partial F}{\partial y_{l}} \circ \pi_{2}\right) \circ \phi$

$=\left(0, g \circ \pi_{2}\right) \circ \phi \in\left(m_{Y} J M_{z}(X)\right)_{D} \circ \phi$. By curve criterion we conclude that

$\left(0, \frac{\partial F}{\partial y_{l}} \circ \pi_{2}\right) \in \overline{\left(m_{Y} J M_{z}(X)\right)_{D}}$ at $\left(x, x^{\prime}\right)$.

Finally, let us to prove that $\left(J M(X)_{Y}\right)_{D} \subseteq \overline{\left(m_{Y} J M_{Z}(X)\right)_{D}}$ at $\left(x, x^{\prime}\right)$. In fact, for all

$i \in\{1, \ldots, n\}$ and $l \in\{1, \ldots k\}$ we have that $\left(0,\left(z_{i} \circ \pi_{1}-z_{i} \circ \pi_{2}\right)\left(\frac{\partial F}{\partial y_{l}} \circ \pi_{2}\right)\right)=$ $\left(z_{i} \circ \pi_{1}-z_{i} \circ \pi_{2}\right) \underbrace{\left(0, \frac{\partial F}{\partial y_{l}} \circ \pi_{2}\right)}_{\in \overline{\left(m_{Y} J M_{z}(X)\right)_{D}}} \in \overline{\left(m_{Y} J M_{z}(X)\right)_{D}}$ and

$$
\left(\frac{\partial F}{\partial y_{l}}\right)_{D}=(\sum_{j=1}^{p}\left(\frac{\partial F_{j}}{\partial y_{l}} \circ \pi_{1}\right) \underbrace{\left(e_{j}, 0\right)}_{\in\left(m_{Y} J M_{z}(X)\right)_{D}})+\underbrace{\left(0, \frac{\partial F}{\partial y_{l}} \circ \pi_{2}\right)}_{\in \overline{\left(m_{Y} J M_{z}(X)\right)_{D}}} \in \overline{\left(m_{Y} J M_{z}(X)\right)_{D}} .
$$

Therefore, $\left(J M(X)_{Y}\right)_{D} \subseteq \overline{\left(m_{Y} J M_{z}(X)\right)_{D}}$ at $\left(x, x^{\prime}\right)$ and the $i L_{m_{Y}}$ and $i L_{A}$ condition are satisfied in $\left(x, x^{\prime}\right)$.

The next result generalizes the Theorem 4.3 of [39] and states that the infinitesimal Lipschitz condition A holds generically along the parameter space $Y$.

Theorem 2.2.11. Consider the setup 2.2.1. Then there exists a dense Zariski open subset $U$ of $Y$ such that the infinitesimal Lipschitz condition A holds for the pair $(X-Y, U \cap Y)$ along $Y$.

Proof. We can write a matrix of generators of $\left(J M_{z}(X)\right)_{D}$ as

$$
\left[\left(J M_{z}(X)\right)_{D}\right]=\left[\begin{array}{cc}
J M_{z}(x) \circ \pi_{1} & 0 \\
J M_{z}(X) \circ \pi_{2} & \left(0,\left(z_{i} \circ \pi_{1}-z_{i} \circ \pi_{2}\right)\left(\frac{\partial F}{\partial z_{s}} \circ \pi_{2}\right)\right)_{i, s=1}^{n}
\end{array}\right]
$$

whose entries are in $\mathscr{O}_{X \times X}$. Since $\left(J M_{z}(X)\right)_{D}$ is a sheaf of submodules of $\mathscr{O}_{X \times X}^{2 p}$ then, choosing $S_{1}, \ldots, S_{2 p}$ as the homogeneous coordinates on $\mathbb{P}^{2 p-1}$, we can consider the sheaf of ideals of $\mathscr{O}_{X \times X \times \mathbb{P}^{2 p-1}}$ induced by $\left(J M_{z}(X)\right)_{D}$, namely $\rho\left(\left(J M_{z}(X)\right)_{D}\right)$, which is generated by the entries of the vector

$$
\left[\begin{array}{llll}
1 & \frac{S_{2}}{S_{1}} & \cdots & \frac{S_{2 p}}{S_{1}}
\end{array}\right] \cdot\left[\left(J M_{z}(X)\right)_{D}\right]
$$

on the chart $U_{1}:=\left\{\left[S_{1}, \ldots, S_{2 p}\right] \in \mathbb{P}^{2 p-1} \mid S_{1} \neq 0\right\}$ which is a dense Zariski open subset of $\mathbb{P}^{2 p-1}$.

Denote by $N:=N B_{\rho\left(\left(J M_{z}(X)\right)_{D}\right)}\left(X \underset{Y}{\times} X \times \mathbb{P}^{2 p-1}\right)$ the normalized blow-up of $X \underset{Y}{\times} X \times$ $\mathbb{P}^{2 p-1}$ with respect to the sheaf of ideals $\rho\left(\left(J M_{Z}(X)\right)_{D}\right)$ of $\mathscr{O}_{X \times X \times \mathbb{P}} \mathbb{P}^{2 p-1}$. Consider the projection map $\pi: N \rightarrow X \underset{Y}{\times} X \times \mathbb{P}^{2 p-1}$ and let $E \subseteq N$ be the normalized exceptional divisor. To prove this theorem we use the Corollary 2.1.16 and the module criterion (see Proposition 3.5 in [48]), i.e, in order to verify the condition $\left(J M(X)_{Y}\right)_{D} \subseteq \overline{\left(J M_{Z}(X)\right)_{D}}$ in a dense Zariski open subset $U$ of $Y$, it suffices to check that on each component of the exceptional divisor, the pullback of the element 
$\rho\left(\left(\frac{\partial F}{\partial y}\right)_{D}\right)$ to the normalized blow-up is in the pullback of $\rho\left(\left(J M_{z}(X)\right)_{D}\right)$, for every coordinate $y$ in the parameter space.

Let $\mathbf{p}: X \subseteq \mathbb{C}^{n} \times Y \rightarrow Y$ be the projection onto $Y$. For each $\ell \in\{1,2\}$ consider the projection map $p_{\ell}: X \underset{Y}{\times} X \times \mathbb{P}^{2 p-1} \rightarrow X$ on the $\ell^{\text {th }}$ factor and $\bar{\pi}_{\ell}: N \rightarrow X$ given $\bar{\pi}_{\ell}:=p_{\ell} \circ \pi$.

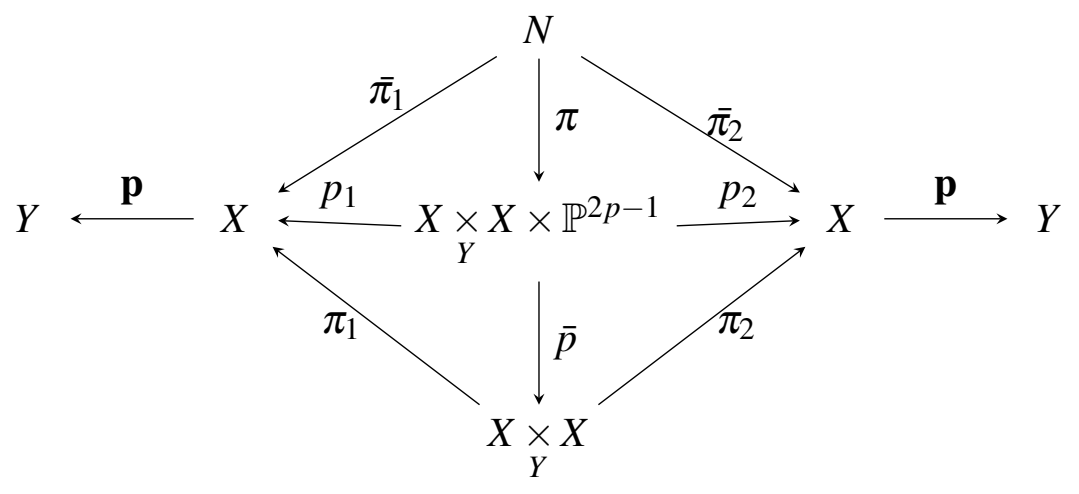

By Proposition 2.2.10 we need only consider those components of the exceptional divisor which projects to $Y$ under the map to $X \underset{Y}{\times} X$. Since we are working over a dense Zariski open subset of $Y$ we may assume that every such components maps surjectively onto $Y$. Since $N$ is a normal space and $E$ has codimension 1 in $N$ then we can work at a point $q$ of the normalized exceptional divisor $E$ such that $E$ is smooth at $q, N$ is smooth at $q$ and the projection to $Y$ is a submersion at $q$. Thus we can choose coordinates $\left(y^{\prime}, u^{\prime}, x^{\prime}\right)$ such that $y^{\prime}=y \circ \mathbf{p}, u^{\prime}$ defines $E$ locally with reduced structure and $\frac{\partial u^{\prime}}{\partial y^{\prime}}=0$, i.e, $u^{\prime}$ and $y^{\prime}$ are independent coordinates. Working on the subset $U_{1} \subset \mathbb{P}^{2 p-1}$, since $X$ is defined by $F$ then the germ of

$$
\left[\begin{array}{llll}
1 & \frac{S_{2}}{S_{1}} & \ldots & \frac{S_{2 p}}{S_{1}}
\end{array}\right] \cdot\left[\begin{array}{c}
F_{1} \circ p_{1} \\
\vdots \\
F_{p} \circ p_{1} \\
F_{1} \circ p_{2} \\
\vdots \\
F_{p} \circ p_{2}
\end{array}\right]=0
$$

is identically zero on $X \underset{Y}{\times} X \times \mathbb{P}^{2 p-1}$. Pull this back to $N$ by $\pi$ and take the partial derivative with respect to $y^{\prime}$ at $q$. We get by the chain rule: 


$$
\left[\begin{array}{lll}
1 & \frac{S_{2}}{S_{1}} \quad \ldots & \frac{S_{2 p}}{S_{1}}
\end{array}\right] \cdot\left[\begin{array}{c}
\frac{\partial F_{1}}{\partial y} \circ \bar{\pi}_{1}+\sum_{i=1}^{n}\left(\frac{\partial F_{1}}{\partial z_{i}} \circ \bar{\pi}_{1}\right)\left(\frac{\partial\left(z_{i} \circ \bar{\pi}_{1}\right)}{\partial y^{\prime}}\right) \\
\vdots \\
\frac{\partial F_{p}}{\partial y} \circ \bar{\pi}_{1}+\sum_{i=1}^{n}\left(\frac{\partial F_{p}}{\partial z_{i}} \circ \bar{\pi}_{1}\right)\left(\frac{\partial\left(z_{i} \circ \bar{\pi}_{1}\right)}{\partial y^{\prime}}\right) \\
\frac{\partial F_{1}}{\partial y} \circ \bar{\pi}_{2}+\sum_{i=1}^{n}\left(\frac{\partial F_{1}}{\partial z_{i}} \circ \bar{\pi}_{2}\right)\left(\frac{\partial\left(z_{i} \circ \bar{\pi}_{2}\right)}{\partial y^{\prime}}\right) \\
\vdots \\
\frac{\partial F_{p}}{\partial y} \circ \bar{\pi}_{2}+\sum_{i=1}^{n}\left(\frac{\partial F_{p}}{\partial z_{i}} \circ \bar{\pi}_{2}\right)\left(\frac{\partial\left(z_{i} \circ \bar{\pi}_{2}\right)}{\partial y^{\prime}}\right)
\end{array}\right]=0
$$

Since $F_{j} \circ \bar{\pi}_{1}=F_{j} \circ \bar{\pi}_{2}=0$ for all $j \in\{1, \ldots, p\}$ then there is no term involving the derivatives of the homogeneous coordinates with respect to $y^{\prime}$. Notice that all $z_{i}$ vanish along $Y$ and $z_{i} \circ \bar{\pi}_{1}$ and $z_{i} \circ \bar{\pi}_{2}$ vanish along $E$ at $q$ then we can assume that the order of vanishing of $z_{1} \circ \bar{\pi}_{\ell}$ is minimal among $\left\{z_{i} \circ \bar{\pi}_{\ell}\right\}$ and that the strict transform of $z_{1} \circ \bar{\pi}_{\ell}$ do not pass through $q$, $\forall \ell \in\{1,2\}$.

By the equation $(\star)$ we have that $\rho\left(\left(\frac{\partial F}{\partial y}\right)_{D}\right) \circ \pi=\left[\begin{array}{llll}1 & \frac{S_{2}}{S_{1}} & \ldots & \frac{S_{2 p}}{S_{1}}\end{array}\right] \cdot\left[\begin{array}{c}\frac{\partial F_{1}}{\partial y} \circ \bar{\pi}_{1} \\ \vdots \\ \frac{\partial F_{p}}{\partial y} \circ \bar{\pi}_{1} \\ \frac{\partial F_{1}}{\partial y} \circ \bar{\pi}_{2} \\ \vdots \\ \frac{\partial F_{p}}{\partial y} \circ \bar{\pi}_{2}\end{array}\right]=-v$,

where

$$
v:=\left[\begin{array}{llll}
1 & \frac{S_{2}}{S_{1}} & \ldots & \frac{S_{2 p}}{S_{1}}
\end{array}\right] \cdot\left[\begin{array}{c}
\sum_{i=1}^{n}\left(\frac{\partial F_{1}}{\partial z_{i}} \circ \bar{\pi}_{1}\right)\left(\frac{\partial\left(z_{i} \circ \bar{\pi}_{1}\right)}{\partial y^{\prime}}\right) \\
\vdots \\
\sum_{i=1}^{n}\left(\frac{\partial F_{p}}{\partial z_{i}} \circ \bar{\pi}_{1}\right)\left(\frac{\partial\left(z_{i} \circ \bar{\pi}_{1}\right)}{\partial y^{\prime}}\right) \\
\sum_{i=1}^{n}\left(\frac{\partial F_{1}}{\partial z_{i}} \circ \bar{\pi}_{2}\right)\left(\frac{\partial\left(z_{i} \circ \bar{\pi}_{2}\right)}{\partial y^{\prime}}\right) \\
\vdots \\
\sum_{i=1}^{n}\left(\frac{\partial F_{p}}{\partial z_{i}} \circ \bar{\pi}_{2}\right)\left(\frac{\partial\left(z_{i} \circ \bar{\pi}_{2}\right)}{\partial y^{\prime}}\right)
\end{array}\right] .
$$

In order to simplify the notation, for each $i \in\{1, \ldots n\}$ define 


$$
w_{i}:=\left[\begin{array}{llll}
1 & S_{2} & \ldots & \frac{S_{2 p}}{S_{1}}
\end{array}\right] \cdot\left[\begin{array}{c}
\left(\frac{\partial F_{1}}{\partial z_{i}} \circ \bar{\pi}_{1}\right)\left(\frac{\partial\left(z_{i} \circ \bar{\pi}_{1}\right)}{\partial y^{\prime}}\right) \\
\vdots \\
\left(\frac{\partial F_{p}}{\partial z_{i}} \circ \bar{\pi}_{1}\right)\left(\frac{\partial\left(z_{i} \circ \bar{\pi}_{1}\right)}{\partial y^{\prime}}\right) \\
\left(\frac{\partial F_{1}}{\partial z_{i}} \circ \bar{\pi}_{2}\right)\left(\frac{\partial\left(z_{i} \circ \bar{\pi}_{1}\right)}{\partial y^{\prime}}\right) \\
\vdots \\
\left(\frac{\partial F_{p}}{\partial z_{i}} \circ \bar{\pi}_{2}\right)\left(\frac{\partial\left(z_{i} \circ \bar{\pi}_{1}\right)}{\partial y^{\prime}}\right)
\end{array}\right]
$$

and

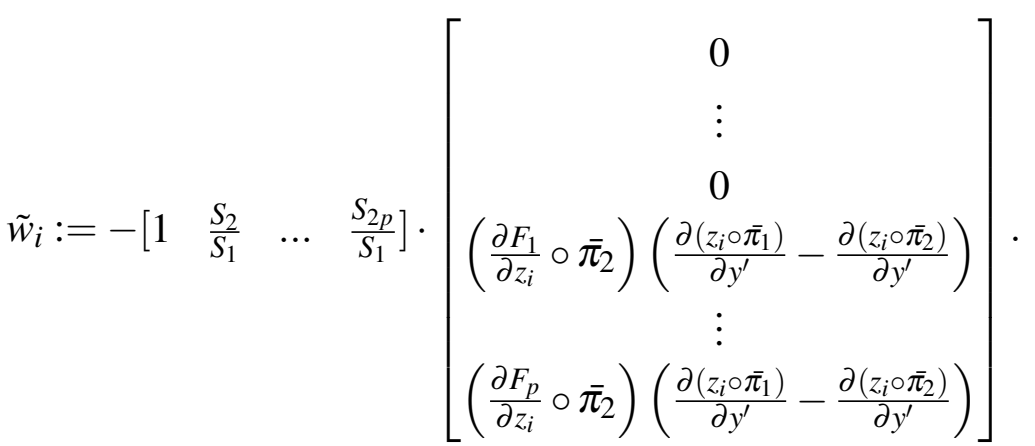

Clearly $v=\sum_{i=1}^{n}\left(w_{i}+\tilde{w}_{i}\right)$. For every $i \in\{1, \ldots, n\}$ we have that $w_{i}=\frac{\partial\left(z_{i} \circ \bar{\pi}_{1}\right)}{\partial y^{\prime}} \pi^{*}\left(\rho\left(\left(\frac{\partial F}{\partial z_{i}}\right)_{D}\right)\right) \in \pi^{*}\left(\rho\left(\left(J M_{z}(X)\right)_{D}\right)\right)$.

Now it suffices to check that $\tilde{w}_{i} \in \pi^{*}\left(\rho\left(\left(J M_{Z}(X)\right)_{D}\right)\right), \forall i \in\{1, \ldots, n\}$. Since the pullback of the ideal $\rho\left(\left(J M_{z}(X)\right)_{D}\right)$ is locally principal then we can work at a point $q$ such that $\pi^{*}\left(\rho\left(\left(J M_{z}(X)\right)_{D}\right)\right)$ is generated by $u^{\prime r}$, a power of $u^{\prime}$. Since $\mathscr{O}_{N, q}$ is a normal ring then the Lemma 1.12 of [64] implies that the ideal $\pi^{*}\left(\rho\left(\left(J M_{z}(X)\right)_{D}\right)\right)$ is integrally closed, i.e, $\overline{\pi^{*}\left(\rho\left(\left(J M_{z}(X)\right)_{D}\right)\right)}=\pi^{*}\left(\rho\left(\left(J M_{z}(X)\right)_{D}\right)\right)$. So, it is enough to prove that $\tilde{w}_{i} \in \overline{\pi^{*}\left(\rho\left(\left(J M_{z}(X)\right)_{D}\right)\right)}$, for all $i \in\{1, \ldots, n\}$. Let $i \in\{1, \ldots, n\}$ be arbitrary. We use the curve criterion. Let $\tilde{\phi}:(\mathbb{C}, 0) \rightarrow$ $(N, q)$ be an analytic curve. We can choose $\tilde{\phi}$ such that $\phi:(\mathbb{C}, 0) \rightarrow\left(\underset{Y}{\times X} X \mathbb{P}^{2 p-1}, \pi(q)\right)$ given by $\phi:=\pi \circ \tilde{\phi}$ meets the dense Zariski open subset $U_{1}, \phi=\left(\phi_{1}, \phi_{2}, \psi\right)$ and $\psi=\left[1, \frac{\psi_{2}}{\psi_{1}}, \ldots, \frac{\psi_{2 p}}{\psi_{1}}\right]$. Further, $\tilde{\phi}$ can be chosen such that $\tilde{\phi}$ is transverse to the component so that $u^{\prime} \circ \tilde{\phi}=t$, where $t$ is the generator of the maximal ideal of $\mathscr{O}_{\mathbb{C}, 0}$. Hence, the pullback of the ideal $\pi^{*}\left(\rho\left(\left(J_{z}(X)\right)_{D}\right)\right)$ is generated by $t^{r}$. Consider the element

$$
\hat{w}_{i}:=-\left[\begin{array}{llll}
1 & S_{2} & \ldots & \frac{S_{2 p}}{S_{1}}
\end{array}\right] \cdot\left[\begin{array}{c}
0 \\
\vdots \\
0 \\
\left(\frac{\partial F_{1}}{\partial z_{i}} \circ \bar{\pi}_{2}\right)\left(z_{i} \circ \bar{\pi}_{1}-z_{i} \circ \bar{\pi}_{2}\right) \\
\vdots \\
\left(\frac{\partial F_{p}}{\partial z_{i}} \circ \bar{\pi}_{2}\right)\left(z_{i} \circ \bar{\pi}_{1}-z_{i} \circ \bar{\pi}_{2}\right)
\end{array}\right] .
$$


Notice that $\left.\hat{w}_{i}=-\pi^{*}\left(\rho(\underbrace{\left(0,\left(z_{i} \circ \pi_{1}-z_{i} \circ \pi_{2}\right)\left(\frac{\partial F}{\partial z_{i}} \circ \pi_{2}\right)\right.}_{\in\left(J M_{z}(X)_{D}\right)})\right)\right) \in \pi^{*}\left(\rho\left(\left(J M_{z}(X)\right)_{D}\right)\right)$. Since $y^{\prime}$ and $u^{\prime}$ are independent coordinates then the order of $\frac{\partial\left(z_{i} \circ \bar{\pi}_{1}\right)}{\partial y^{\prime}}-\frac{\partial\left(z_{i} \circ \bar{\pi}_{2}\right)}{\partial y^{\prime}}$ in $u^{\prime}$ is the same as the order of $z_{i} \circ \bar{\pi}_{1}-z_{i} \circ \bar{\pi}_{2}$ in $u^{\prime}$. Then the pullback of both have the same order in $t$, so there exists an invertible element $\alpha_{i} \in \mathscr{O}_{\mathbb{C}, 0}$ such that

$$
\tilde{\phi}^{*}\left(\frac{\partial\left(z_{i} \circ \bar{\pi}_{1}\right)}{\partial y^{\prime}}-\frac{\partial\left(z_{i} \circ \bar{\pi}_{2}\right)}{\partial y^{\prime}}\right)=\alpha_{i}\left(\tilde{\phi}^{*}\left(z_{i} \circ \bar{\pi}_{1}-z_{i} \circ \bar{\pi}_{2}\right)\right)
$$

Hence, $\tilde{\phi}^{*}\left(\tilde{w}_{i}\right)=-\sum_{j=1}^{p}\left(\frac{\partial F_{j}}{\partial z_{i}} \circ \phi_{2}\right)\left(\tilde{\phi}^{*}\left(\frac{\partial\left(z_{i} \circ \overline{\pi_{1}}\right)}{\partial y^{\prime}}-\frac{\partial\left(z_{i} \circ \bar{\pi}_{2}\right)}{\partial y^{\prime}}\right)\right) \frac{\psi_{p+j}}{\psi_{1}}$

$=\alpha_{i} \tilde{\phi}^{*}\left(\hat{w}_{i}\right) \in \tilde{\phi}^{*}\left(\pi^{*}\left(\rho\left(\left(J M_{z}(X)\right)_{D}\right)\right)\right)$.

Therefore, $\tilde{w}_{i} \in \overline{\pi^{*}\left(\rho\left(\left(J M_{z}(X)\right)_{D}\right)\right)}$, for all $i \in\{1, \ldots, n\}$ which finishes the proof.

\subsection{The genericity theorem applied in a family of hyper- plane sections}

Given $X$ an analytic variety with isolated singularity at the origin, we can consider the sections of $X$ by hyperplanes. One natural question is if there exists a generic set of hyperplanes for which the family of hyperplanes sections satisfies the infinitesimal Lipschitz condition A. We will show this is true. First, we recall some important notions in order to make precise statements. Fore more details see [44].

Let us work on the Grassmanian modification of $X=f^{-1}(0)$, defined by an analytic map $f:\left(\mathbb{C}^{n}, 0\right) \rightarrow\left(\mathbb{C}^{p}, 0\right), X$ with isolated singularity at the origin, $n \geq p$.

For each $y=\left[y_{1}, \ldots, y_{n}\right] \in \mathbb{P}^{n-1}$, consider the hyperplane on $\mathbb{C}^{n}$ given by

$$
H_{y}:=\left\{z=\left(z_{1}, \ldots, z_{n}\right) \in \mathbb{C}^{n} \mid z \cdot y:=\sum_{i=1}^{n} z_{i} y_{i}=0\right\} .
$$

Let $E_{n-1}$ be the canonical bundle over $\mathbb{P}^{n-1}$, i.e,

$$
E_{n-1}:=\left\{(z, y) \in \mathbb{C}^{n} \times \mathbb{P}^{n-1} \mid z \in H_{y}\right\}
$$

Consider the projection map $\beta: E_{n-1} \rightarrow \mathbb{C}^{n}$. We call $\tilde{X}:=\beta^{-1}(X)$ the $(\mathbf{n}-\mathbf{1})$-Grassmanian modification of $\mathbf{X}$. Here we will simply refer to the $(n-1)$-modification as the Grassmanian modification of $X$. We can see $\mathbb{P}^{n-1}$ embedded into $E_{n-1}$ as the zero section of the bundle $E_{n-1}$, which allows us to think of $0 \times \mathbb{P}^{n-1}$ as a stratum of $\tilde{X}$. Note that the projection to $0 \times \mathbb{P}^{n-1}$ makes $\tilde{X}$ a family of analytic sets with $0 \times \mathbb{P}^{n-1}$ as the parameter space, which we denote by $Y$. The members of this family are just $\left\{H_{y} \cap X\right\}$ as $y$ varies in $\mathbb{P}^{n-1}$. 


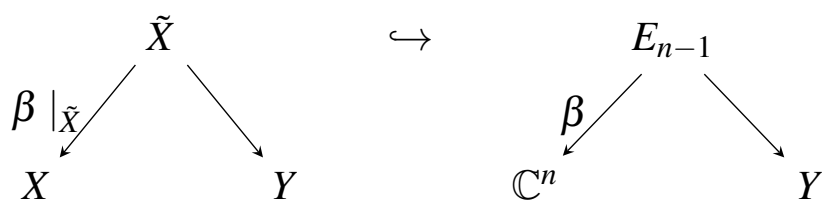

Consider the chart

$$
U_{n}:=\left\{\left[y_{1}, \ldots, y_{n}\right] \in \mathbb{P}^{n-1} \mid y_{n} \neq 0\right\}=\left\{\left[y_{1}, \ldots, y_{n-1},-1\right] \mid\left(y_{1}, \ldots, y_{n-1}\right) \in \mathbb{C}^{n-1}\right\} \equiv \mathbb{C}^{n-1}
$$

which is a dense Zariski open subset of $\mathbb{P}^{n-1}$. Working on the dense Zariski open subset $E_{n-1} \cap\left(\mathbb{C}^{n} \times U_{n}\right)$ of $E_{n-1}$, we have local coordinates given by $\left(z_{1}, \ldots, z_{n}, y_{1}, \ldots, y_{n-1}\right)$. In these coordinates, the projection map $\beta$ satisfies the equation

$$
\beta\left(z_{1}, \ldots, z_{n}, y_{1}, \ldots, y_{n-1}\right)=\left(z_{1}, \ldots, z_{n-1}, \sum_{i=1}^{n-1} y_{i} z_{i}\right) .
$$

Consider the analytic map

$$
\begin{array}{ccc}
F: E_{n-1} \cap\left(\mathbb{C}^{n} \times U_{n}\right) & \rightarrow & \mathbb{C}^{p} \\
(z, y) & \mapsto f \circ \beta(z, y)
\end{array}
$$

Thus, $F^{-1}(0)=\beta^{-1}\left(f^{-1}(0)\right)=\beta^{-1}(X)=\tilde{X}$, hence $\tilde{X}$ is defined by $F$. For each $y=\left(y_{1}, \ldots, y_{n-1}\right)$ $\equiv\left[y_{1}, \ldots, y_{n-1},-1\right] \in U_{n}$, let $F_{y}: \mathbb{C}^{n} \rightarrow \mathbb{C}^{p}$ given by $F_{y}(z):=F(z, y)$ and let $\tilde{X}_{y}:=F^{-1}(0)$. In these coordinates, clearly $\tilde{X}_{y}=\left(f^{-1}(0)\right) \cap H_{y}=X \cap H_{y}$. Therefore, $F$ defines the family of sections of $X$ by the hyperplanes $H_{y}$, as $y$ varies on the dense Zariski open subset $U_{n}$ of $\mathbb{P}^{n-1}$.

The next result generalizes the Theorem 4.4 of [39].

Theorem 2.3.1. Suppose $(X, 0)$ is a germ of an isolated singularity analytic variety defined by an analytic map-germ $f:\left(\mathbb{C}^{n}, 0\right) \rightarrow\left(\mathbb{C}^{p}, 0\right), n \geq p$, and consider the Grassmanian modification $\tilde{X}$ of $X$. Then, there exists a non-empty Zariski open subset $U$ of $\mathbb{P}^{n-1}$, such that the $i L_{A}$ condition holds for the pair $(\tilde{X}-U, U)$ along $U$.

Proof. As we have seen, $\tilde{X}$ is a family defined by the above analytic map $F$.

Let us prove that $\tilde{X}_{y}$ has isolated singularity at $(0, y)$ for all $y$ varying in a non-empty Zariski open subset $U^{\prime}$ of $U_{n}$. In fact, we already know that the set of limiting tangent hyperplanes of $X$ at the origin is a Zariski proper closed subset of $\mathbb{P}^{n-1}$. Call this set $W$. Let $U^{\prime}:=U_{n}-\left(W \cap U_{n}\right)$. Since $\mathbb{P}^{n-1}$ is irreducible then $U_{n}$ is irreducible. Since $U_{n}$ is a dense subset of $\mathbb{P}^{n-1}$ then $W \cap U_{n}$ also is a proper Zariski closed subset of $U_{n}$, hence $U^{\prime}$ is a dense Zariski open subset of $U_{n}$. Let $y \in U^{\prime}$. We want to show that $(0, y)$ is an isolated singularity of $\tilde{X}_{y}$. By hypothesis, $H_{y}$ is not a limiting tangent hyperplane of $X$ at the origin, and by Lemma 4.1 (a) of [48] we have that $\overline{J M(X)_{H_{y}}}=\overline{J M(X)}$ at the origin, where $J M(X)_{H_{y}}:=\left\{\frac{\partial f}{\partial v} \mid v \in H_{y}\right\}$. Thus, in a neighborhood of the origin, the generic rank of $J M(X)$ and $J M(X)_{H_{y}}$ is the same. Thus, if we take 
$z$ in this neighborhood, such that $z \in H_{y}, z \neq 0$ then the generic rank of $J M\left(\tilde{X}_{y}\right)=J M\left(X \cap H_{y}\right)$ at $z$ is the generic rank of $J M(X)_{H_{y}}$ at $z$, which is the generic rank of $J M(X)$ at $z$. Since $z \neq 0$ and $X$ has isolated singularity at the origin then we can choose this neighborhood so that $z$ is a non-singular point of $X$, which implies that $z$ is not a singular point of $\tilde{X}_{y}$. Therefore, $\tilde{X}_{y}$ has isolated singularity at the origin, for all $y \in U^{\prime}$.

Now, the existence of $U$ follows from the Theorem 2.2.11.

Let us go back to the discussion before the last theorem. We have seen that $\tilde{X}$ is defined by the map $F: E_{n-1} \cap\left(\mathbb{C}^{n} \times U_{n}\right) \rightarrow \mathbb{C}^{p}$ given by $F(z, y)=f \circ \beta(z, y)$. From the chain rule we have that

$$
\left[d F_{(z, y)}\right]=\left[d f_{\beta(z, y)}\right] \cdot\left[\begin{array}{cccccccc}
1 & 0 & \ldots & 0 & 0 & 0 & \ldots & 0 \\
0 & 1 & \ldots & 0 & 0 & 0 & \ldots & 0 \\
0 & 0 & \ldots & 0 & 0 & 0 & \ldots & 0 \\
\vdots & \vdots & & \vdots & \vdots & \vdots & & \vdots \\
0 & 0 & \ldots & 1 & 0 & 0 & \ldots & 0 \\
y_{1} & y_{2} & \ldots & y_{n-1} & 0 & z_{1} & \ldots & z_{n-1}
\end{array}\right]
$$

which implies that $\frac{\partial F}{\partial y_{i}}=z_{i}\left(\frac{\partial f}{\partial z_{n}} \circ \beta\right)$ and $\frac{\partial F}{\partial z_{i}}=\frac{\partial f}{\partial z_{i}} \circ \beta+\sum_{j=1}^{n-1} y_{j}\left(\frac{\partial f}{\partial z_{n}} \circ \beta\right)$, for all $i \in\{1, \ldots, n-1\}$, and $\frac{\partial F}{\partial z_{n}}=0$. Thus, we have immediately the next result, which is a generalization of the Corollary 4.5 of [39].

Corollary 2.3.2. The point $(0, P) \in E_{n-1} \cap\left(\mathbb{C}^{n} \times U_{n}\right)$ belongs to the Zariski open subset of the last theorem if and only if $\left(z_{i}\left(\frac{\partial f}{\partial z_{n}} \circ \beta\right)\right)_{D} \in \overline{\left(J M_{z}(\tilde{X})\right)_{D}}$ at $(0, P)$, for all $i \in\{1, \ldots, n-1\}$.

The corollary tell us that in order to check if a hyperplane is generic, it is enough to verify that for all curves $\phi_{1}, \phi_{2}$, tangent to $P$ at the origin with lifts $\tilde{\phi}_{1}, \tilde{\phi}_{2}$, respectively, with $\phi:=\left(\phi_{1}, \phi_{2}\right)$ and $\tilde{\phi}:=\left(\tilde{\phi}_{1}, \tilde{\phi}_{2}\right)$, we have that $\left(z_{i}\left(\frac{\partial f}{\partial z_{n}} \circ \beta\right)\right) \circ \phi$ is an $\mathscr{O}_{\mathbb{C}, 0}$-linear combination of $\left\{\left(\frac{\partial f}{\partial z_{s}} \circ \phi\right)+\left(\sum_{j=1}^{n-1} y_{j}\left(\frac{\partial f}{\partial z_{n}} \circ \beta\right)\right) \circ \tilde{\phi}\right\}_{s=1}^{n-1}$, for all $i \in\{1, \ldots, n-1\}$.

In [39] Gaffney gave a description of these generic hyperplanes using analytic invariants in the jacobian ideal case. Now we generalize this description for the jacobian module case. For the rest of this section we assume that the hyperplanes $H_{y}$ are not limiting tangent hyperplanes of $(X, 0)$. As we have seen, this implies that $\tilde{X}_{y}=X \cap H_{y}$ has isolated singularity at the origin and $\overline{J M(X)_{H_{y}}}=\overline{J M(X)}$ at the origin.

The invariants that we use here appeared earlier in section 2.1. Since $\tilde{X}_{y}$ has isolated singularity at the origin then by Corollary 2.1.27 the multiplicity of the pair $e\left(\left(\operatorname{JM}\left(\tilde{X}_{y}\right)\right)_{D},\left(\overline{J M\left(\tilde{X}_{y}\right)}\right)_{D}\right)$ is well defined.

These invariants have been used in this context before. As pointed out by Gaffney in [39], for ICIS singularities, and more generally in [43], to check for whether or not a hyperplane is in 
the generic set of hyperplanes for which the hyperplane sections form a Whitney equisingular family, you can use the multiplicity of the pair $\left(J M\left(\tilde{X}_{y}\right), \mathscr{O}_{\tilde{X}_{y}}^{p}\right)$, which is the Buchsbaum-Rim multiplicity $e\left(J M\left(\tilde{X}_{y}\right)\right)$.

The hyperplane is generic if this multiplicity is minimal, and the minimal number is the sum of the Milnor number of $X \cap H_{y}$ and $X \cap H_{y} \cap H_{y^{\prime}}$, where $H_{y}$ and $H_{y^{\prime}}$ are generic hyperplanes.

The proof that the minimal value of $e\left(\left(J M\left(\tilde{X}_{y}\right)\right)_{D},\left(\overline{J M\left(\tilde{X}_{y}\right)}\right)_{D}\right)$ identifies generic hyperplanes will be done using the Multiplicity Polar Theorem (see Corollary 1.4 [45]). Now we identify the modules we will use.

We will work on the fibered product $\tilde{X} \underset{\mathbb{P}^{n-1}}{\times} \tilde{X} \subseteq X \times \mathbb{P}^{n-1} \times X$. Let $N:=\left(\beta^{*}(\overline{J M(X)})\right)_{D}$ and $M:=\left(J M_{z}(\tilde{X})\right)_{D}$, considering $\tilde{X}$ defined by the analytic map $F: E_{n-1} \cap\left(\mathbb{C}^{n} \times U_{n}\right) \rightarrow \mathbb{C}^{p}$, given by $F(z, y)=f \circ \beta(z, y)$. Clearly $M$ restricted to the fiber of the family $\tilde{X}$ over the hyperplane $H_{y}$ is just $\left(J M\left(X \cap H_{y}\right)\right)_{D}$ and $N$ restricted to $H_{y}$ is $\left(\left.\overline{J M(X)}\right|_{H_{y}}\right)_{D}$. Further, since we are assuming that $H_{y}$ is not a limiting tangent hyperplane of $(X, 0)$ then $\left.\overline{J M(X)}\right|_{H_{y}}=\overline{J M(X)_{H_{y}}}$, hence $N$ restricted to $H_{y}$ is $\left(\overline{J M\left(\tilde{X}_{y}\right)}\right)_{D}$. Therefore, the multiplicity of the pair $\left(\left.M\right|_{H_{y}},\left.N\right|_{H_{y}}\right)$ is the same as $e\left(\left(J M\left(\tilde{X}_{y}\right)\right)_{D},\left(\overline{J M\left(\tilde{X}_{y}\right)}\right)_{D}\right)$.

The next result is a generalization of the Theorem 4.6 of [39].

Theorem 2.3.3. Under the above notations, let $U$ be the set of hyperplanes which are not limiting tangent hyperplanes of $(X, 0)$. Suppose that $N$ has no polar variety with the same codimension of $U$. Then:

a) The map

$$
\begin{array}{rlr}
U & \rightarrow & \mathbb{Z} \\
H_{y} & \mapsto & e\left(\left(J M\left(\tilde{X}_{y}\right)\right)_{D},\left(\overline{J M\left(\tilde{X}_{y}\right)}\right)_{D}\right)
\end{array}
$$

is upper semicontinuous on $U$;

b) The $i L_{A}$ condition holds along $U$ at a hyperplane $H_{y}$ for which the value of $e\left(\left(J M\left(\tilde{X}_{y}\right)\right)_{D},\left(\overline{J M\left(\tilde{X}_{y}\right)}\right)_{D}\right)$ is minimal.

Proof. (a) By the definition of $U,\left(\overline{J M\left(\tilde{X}_{y}\right)}\right)_{D}$ is the restriction of $N$ to the fiber $\tilde{X}_{y}=X \cap H_{y}$. Since $N$ has no polar variety with the same codimension of $U$ then the multiplicity polar theorem implies that $e\left(\left(J M\left(\tilde{X}_{y}\right)\right)_{D},\left(\overline{J M\left(\tilde{X}_{y}\right)}\right)_{D}\right)$ is upper semicontinuous.

(b) Suppose $H_{y} \in U$ gives the minimal value of the multiplicity. Since this value already is minimal then it cannot go down, hence it must be constant. This implies that the polar variety of $M$ of the same codimension as $U$ is empty, which puts restrictions on the size of the fiber of $\operatorname{Proj}(\mathscr{R}(M))$. We already know that $\left\{\frac{\partial F}{\partial y_{i}}\right\}_{i=1}^{n-1}$ are in $\bar{M}$ generically. Since the dimension of the fiber of $\operatorname{Proj}(\mathscr{R}(M))$ is bounded then by Theorem A1 of [62] we have that $\left\{\frac{\partial F}{\partial y_{i}}\right\}_{i=1}^{n-1}$ are in $\bar{M}$ at $H_{y}$, which finishes the proof. 
In order to check the hypothesis over $N$, we have to check if the fiber dimension of $\operatorname{Proj}(\mathscr{R}(N))$ is small enough so that $N$ has no polar variety of the same codimension of $U$. 
CHAPTER

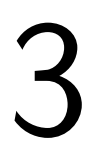

\section{THE LIPSCHITZ SATURATION OF A \\ MODULE}

Motivated by some properties that the Lipschitz saturation has on the ideal case, we define some kinds of Lipschitz saturation for modules.

\subsection{The Lipschitz saturation of a module and basic prop- erties}

Let $X \subseteq \mathbb{C}^{n}$ be an analytic set and let $\mathscr{M}$ be a sheaf of $\mathscr{O}_{X}$-submodules of $\mathscr{O}_{X}^{p}$ generated by global sections $\left\{g_{1}, \ldots, g_{r}\right\}$. Consider the ideal sheaf $\rho(\mathscr{M})$ on $X \times \mathbb{P}^{p-1}$ induced by $\mathscr{M}$. Then $\rho(\mathscr{M})$ is generated by $\left\{\rho\left(g_{1}\right), \ldots, \rho\left(g_{r}\right)\right\}$, so we can consider the blowup $B_{\rho(\mathscr{M})}\left(X \times \mathbb{P}^{p-1}\right) \subseteq$ $\left(X \times \mathbb{P}^{p-1}\right) \times \mathbb{P}^{r-1}$ and the blowup map

$$
\pi: B_{\rho(\mathscr{M})}\left(X \times \mathbb{P}^{p-1}\right) \rightarrow X \times \mathbb{P}^{p-1}
$$

Let $T_{1}, \ldots, T_{p}$ and $S_{1}, \ldots, S_{r}$ be the homogeneous coordinates of $\mathbb{P}^{p-1}$ and $\mathbb{P}^{r-1}$, respectively.

Definition 3.1.1. Let $x \in X$. The 1-Lipschitz saturation of $\mathscr{M}$ at $\mathbf{x}$ is denoted by $\left(\mathscr{M}_{S_{1}}\right)_{x}$ and is defined by

$$
\left(\mathscr{M}_{S_{1}}\right)_{x}:=\left\{h \in \mathscr{O}_{X, x}^{p} \mid \rho(h) \in(\rho(\mathscr{M}))_{S} \text { at all }\left(x,\left[t_{1}, \ldots, t_{p}\right]\right) \in V(\rho(\mathscr{M}))\right\} .
$$

In the family case, the 1-Lipschitz saturation of $\mathscr{M}$ at $\mathbf{X} \in \mathbf{X}$ relative to $\mathbf{Y}$ is defined as above taking the Lipschitz saturation of $\rho(\mathscr{M})$ relative to $Y$.

Unless we say the opposite, we denote by $M_{S_{1}}$ the stalk of the sheaf $\mathscr{M}_{S_{1}}$ at an arbitrary point of $X$. 
Write $h=\left(h_{1}, \ldots, h_{p}\right)$ and each generator $g_{j}=\left(g_{1 j}, \ldots, g_{p j}\right)$. Suppose $t_{1} \neq 0$ and take $\left(x,\left[t_{1}, \ldots, t_{p}\right],\left[s_{1}, \ldots, s_{r}\right]\right) \in \pi^{-1}\left(\left(x,\left[t_{1}, \ldots, t_{p}\right]\right)\right)$, with $s_{1} \neq 0$. Then, $\pi^{*}(\rho(\mathscr{M}))$ is locally generated by $\rho\left(g_{1}\right) \circ \pi$, and $\rho(h) \in(\rho(\mathscr{M}))_{S}$ at $\left(x,\left[t_{1}, \ldots, t_{p}\right]\right)$ if and only if $\frac{\rho(h) \circ \pi}{\rho\left(g_{1}\right) \circ \pi}$ is Lipschitz with respect to the system of coordinates $z_{1}, \ldots, z_{n}, \frac{T_{1}}{T_{1}}, \ldots, \frac{T_{p}}{T_{1}}, \frac{S_{1}}{S_{1}}, \ldots, \frac{S_{r}}{S_{1}}$.

Remark 3.1.2. $\mathscr{M}_{S_{1}}$ is the Lipschitz saturation of an ideal if $p=1$. In fact, in this case $\rho(h)=h$, $\forall h \in \mathscr{O}_{X, x}^{p}$ and $\rho(\mathscr{M})=\mathscr{M}$, and the condition $\rho(h) \in(\rho(\mathscr{M}))_{S}$ at all $(x,[1]) \in V(\rho(\mathscr{M}))$ is equivalent to $h \in \mathscr{M}_{S}$ at $x$. Hence, $\mathscr{M}_{S_{1}}=\mathscr{M}_{S}$ at $x$ if $\mathscr{M}$ is an ideal sheaf of $\mathscr{O}_{X}$.

Proposition 3.1.3. Let $\mathscr{M}$ be a sheaf of $\mathscr{O}_{X}$-submodules of $\mathscr{O}_{X}^{p}$. Then:

a) $\mathscr{M}_{S_{1}}$ is an $\mathscr{O}_{X}$-submodule of $\mathscr{O}_{X}^{p}$;

b) $M \subseteq M_{S_{1}} \subseteq \bar{M}$. In particular, $M$ is a reduction of $M_{S_{1}}$ and, if $M$ has finite colenght in $M_{S_{1}}$ then $e\left(M, M_{S_{1}}\right)=0$.

Proof. Let $x \in X$ be an arbitrary point.

(a) Let $h, h^{\prime} \in \mathscr{M}_{S_{1}}$ at $x$ and $\alpha \in \mathscr{O}_{X, x}$. So, in every point $\left(x,\left[t_{1}, \ldots, t_{p}\right]\right) \in V(\rho(\mathscr{M}))$ we have $\rho\left(\alpha h+h^{\prime}\right)=\alpha \rho(h)+\rho\left(h^{\prime}\right) \in(\rho(\mathscr{M}))_{S}$. So, $\alpha h+h^{\prime} \in \mathscr{M}_{S_{1}}$ at $x$, which finishes the proof of (a).

(b) It is pretty obvious that $M \subseteq M_{S_{1}}$. Let us to prove that $M_{S_{1}} \subseteq \bar{M}$.

Let $h \in M_{S_{1}}$ at $x$. So $\rho(h) \in(\rho(M))_{S}$ in every point $\left(x,\left[t_{1}, \ldots, t_{p}\right]\right) \in V(\rho(M))$. By Proposition 2.1 of [40] we have $(\rho(M))_{S} \subseteq \overline{\rho(M)}$, hence $\rho(h) \in \overline{\rho(M)}$ in every point $\left(x,\left[t_{1}, \ldots, t_{p}\right]\right) \in$ $V(\rho(M))$. By Proposition 3.4 of [48] we conclude that $h \in \bar{M}$ at $x$. Therefore, $M_{S_{1}} \subseteq \bar{M}$.

Definition 3.1.4. Consider the Setup 2.2.1. We define the infinitesimal Lipschitz conditions with respect to the 1-Lipschitz saturation of a module.

- The pair $(X, Y)$ satisfies the 1-infinitesimal Lipschitz condition $\mathbf{m}_{\mathbf{Y}}\left(\mathbf{1}-\mathbf{i} \mathbf{L}_{\mathbf{m}_{\mathbf{Y}}}\right)$ at $\mathbf{x} \in \mathbf{X}$ if $J M(X)_{Y} \subseteq\left(m_{Y} J M_{Z}(X)\right)_{S_{1}}$ at $x$;

- The pair $(X, Y)$ satisfies the 1-infinitesimal Lipschitz condition $A\left(1-\mathbf{i L}_{\mathbf{A}}\right)$ at $\mathbf{x} \in \mathbf{X}$ if $J M(X)_{Y} \subseteq\left(J M_{z}(X)\right)_{S_{1}}$ at $x$.

Here we consider the 1-Lipschitz saturation relative to the parameter space Y. Notice that 1-i $L_{m_{Y}}$ implies $1-i L_{A}$.

Corollary 3.1.5. Consider the Setup 2.2.1. If $x=(z, y) \in X$ with $z \neq 0$ then 1-i $L_{m_{Y}}$ and 1-iLA hold at $x$.

Proof. By Lemma 2.2.8 we have that $J M(X)_{Y} \subseteq m_{Y} J M_{z}(X)$ at $x$. By Proposition 3.1.3 the inclusion $m_{Y} J M_{z}(X) \subseteq\left(m_{Y} J M_{z}(X)\right)_{S_{1}}$ holds and therefore the condition 1-iL $L_{m_{Y}}$ holds at $x$. In particular, $1-i L_{A}$ holds at $x$. 
Thus, it remains to investigate the 1-infinitesimal Lipschitz conditions along the parameter space $Y \equiv 0 \times Y$.

Definition 3.1.6. The 2-Lipschitz saturation of the submodule $M \subseteq \mathscr{O}_{X, x}^{p}$ is denoted by $M_{S_{2}}$, and is defined by

$$
M_{S_{2}}:=\left\{h \in \mathscr{O}_{X, x}^{p} \mid h_{D} \in \overline{M_{D}} \text { at }(x, x)\right\} .
$$

In the family case, the 2-Lipschitz saturation of $\mathrm{M}$ at $\mathbf{x} \in \mathbf{X}$ relative to $\mathrm{Y}$ is defined as above taking the double relative to $Y$.

If $\mathscr{M}$ is an $\mathscr{O}_{X}$-submodule of $\mathscr{O}_{X}^{p}$ then we can consider the sheaf of $\mathscr{O}_{X}$-modules $\mathscr{M}_{S_{2}}$ where each $\operatorname{stalk}\left(\mathscr{M}_{S_{2}}\right)_{x}$ is $\left(\mathscr{M}_{x}\right)_{S_{2}}$.

Unless we say the opposite, we denote by $M_{S_{2}}$ the stalk of the sheaf $\mathscr{M}_{S_{2}}$ at an arbitrary point of $X$.

Remark 3.1.7. $M_{S_{2}}$ is the Lipschitz saturation of an ideal if $p=1$. In fact, by Theorem 1.4.2 we have that $h \in M_{S_{2}}$ if and only if $h_{D} \in \overline{M_{D}}$ if and only if $h \in M_{S}$. Therefore, $M_{S_{2}}=M_{S}$ if $M$ is an ideal of $\mathscr{O}_{X, x}^{p}$.

Proposition 3.1.8. Let $\mathscr{M}$ be a sheaf of $\mathscr{O}_{X}$-submodules of $\mathscr{O}_{X}^{p}$. Then:

a) $\mathscr{M}_{S_{2}}$ is an $\mathscr{O}_{X}$-submodule of $\mathscr{O}_{X}^{p}$;

b) $M \subseteq M_{S_{2}} \subseteq \bar{M}$. In particular, $M$ is a reduction of $M_{S_{2}}$ and, if $M$ has finite colenght in $M_{S_{2}}$ then $e\left(M, M_{S_{2}}\right)=0$.

The same result holds in the family case.

Proof. Let $x \in X$ be an arbitrary point.

(a) Let $\alpha \in \mathscr{O}_{X, x}$ and $h, h^{\prime} \in \mathscr{M}_{S_{2}}$ at $x$.

Claim: $\left(0,\left(\alpha \circ \pi_{1}-\alpha \circ \pi_{2}\right)\left(h \circ \pi_{2}\right)\right) \in \overline{M_{D}}$ at $(x, x)$. In fact,

let $\phi:(\mathbb{C}, 0) \rightarrow(X \times X,(x, x))$ be an arbitrary curve. Since $h_{D} \in \overline{M_{D}}$ then by Proposition 2.1.12 (a) we have that $h \in \bar{M}$ at $x$. If we write $\phi=\left(\phi_{1}, \phi_{2}\right)$, we have that $h \circ \phi_{2} \in M \circ \phi_{2}$, so we can write $h \circ$ $\phi_{2}=\sum \alpha_{j}\left(h_{j} \circ \phi_{2}\right)$, with $\alpha_{j} \in \mathscr{O}_{\mathbb{C}, 0}$ and $h_{j} \in M$. By the Lemma 2.1.2(b) we have that $\left(0,\left(\alpha \circ \pi_{1}-\right.\right.$ $\left.\left.\alpha \circ \pi_{2}\right)\left(h_{j} \circ \pi_{2}\right)\right) \in M_{D}$, for all $j$. Thus, we get: $\left(0,\left(\alpha \circ \pi_{1}-\alpha \circ \pi_{2}\right)\left(h \circ \pi_{2}\right)\right) \circ \phi=\left(0,\left(\alpha \circ \phi_{1}-\alpha \circ\right.\right.$ $\left.\left.\phi_{2}\right)\left(h \circ \phi_{2}\right)\right)=\left(0,\left(\alpha \circ \phi_{1}-\alpha \circ \phi_{2}\right)\left(\sum \alpha_{j}\left(h_{j} \circ \phi_{2}\right)\right)=\sum \alpha_{j}(\underbrace{\left(0,\left(\alpha \circ \pi_{1}-\alpha \circ \pi_{2}\right)\left(h_{j} \circ \pi_{2}\right)\right.}_{\in M_{D}}) \circ \phi\right) \in$ $M_{D} \circ \phi$, which proves the claim.

Finally, we have that $h_{D}, h_{D}^{\prime} \in \overline{\mathscr{M}_{D}}$ at $(x, x)$, hence: $\left(\alpha h+h^{\prime}\right)_{D}=\left(\alpha \circ \pi_{1}\right) h_{D}+\left(0,\left(\alpha \circ \pi_{1}-\alpha \circ \pi_{2}\right)\left(h \circ \pi_{2}\right)\right)+h_{D}^{\prime}$, and each factor on the right hand side belongs to $\overline{\mathscr{M}}_{D}$ at $(x, x)$, so the proof of (a) is done. 
(b) If $h \in \mathscr{M}$ at $x$ then $h_{D} \in \mathscr{M}_{D} \subseteq \overline{\mathscr{M}}_{D}$ at $(x, x)$, so $h \in \mathscr{M}_{S_{2}}$ at $x$. Therefore, $M \subseteq M_{S_{2}}$.

Furthermore, if $h \in \mathscr{M}_{S_{2}}$ at $x$ then $h_{D} \in \overline{\mathscr{M}}_{D}$ at $(x, x)$, and by Proposition 2.1.12 (a) we have that $h \in \overline{\mathscr{M}}$ at $x$. Therefore, $M_{S_{2}} \subseteq \bar{M}$.

Definition 3.1.9. Consider the Setup 2.2.1. We define the infinitesimal Lipschitz conditions with respect to the 2-Lipschitz saturation of a module.

- The pair $(X, Y)$ satisfies the 2-infinitesimal Lipschitz condition $\mathbf{m}_{\mathbf{Y}}\left(2-\mathbf{i L}_{\mathbf{m}_{\mathbf{Y}}}\right)$ at $\mathbf{x} \in \mathbf{X}$ if $J M(X)_{Y} \subseteq\left(m_{Y} J M_{Z}(X)\right)_{S_{2}}$ at $x$;

- The pair $(X, Y)$ satisfies the 2-infinitesimal Lipschitz condition $A\left(2-i L_{\mathbf{A}}\right)$ at $\mathbf{x} \in \mathbf{X}$ if $J M(X)_{Y} \subseteq\left(J M_{z}(X)\right)_{S_{2}}$ at $x$.

Here we consider the 2-Lipschitz saturation relative to the parameter space Y. Notice that 2-i $L_{m_{Y}}$ implies $2-i L_{A}$.

Clearly the above definition is equivalent to the Definition 2.2.2.

Corollary 3.1.10. Consider the Setup 2.2.1. If $x=(z, y) \in X$ with $z \neq 0$ then 2-i $L_{m_{Y}}$ and $2-i L_{A}$ hold at $x$. Furthermore, there exists a dense Zariski open subset $U$ of $Y$ such the 2-i $L_{A}$ holds in $(0, y)$, for all $y \in U$.

Proof. By Lemma 2.2.8 we have that $J M(X)_{Y} \subseteq m_{Y} J M_{z}(X)$ at $x$. By Proposition 3.1.8 the inclusion $m_{Y} J M_{z}(X) \subseteq\left(m_{Y} J M_{z}(X)\right)_{S_{2}}$ holds and therefore the condition 2-iL $L_{m_{Y}}$ holds at $x$. In particular, 2-i $i L_{A}$ holds at $x$.

The second statement is a consequence of the Theorem 2.2.11.

Before to define the third Lipschitz saturation, Let us fix some notations.

For each $\psi: X \rightarrow \operatorname{Hom}\left(\mathbb{C}^{p}, \mathbb{C}\right)$ analytic map, $\psi=\left(\psi_{1}, \ldots, \psi_{p}\right)$ and $h=\left(h_{1}, \ldots, h_{p}\right) \in \mathscr{O}_{X}^{p}$, we define $\psi \cdot h \in \mathscr{O}_{X}$ given by $(\psi \cdot h)(z):=\sum_{i=1}^{p} \psi_{i}(z) h_{i}(z)$. We define $\psi \cdot M$ as the ideal of $\mathscr{O}_{X}$ generated $\{\psi \cdot h \mid h \in M\}$.

Lemma 3.1.11. Under the above notation, we have the following properties:

a) $\psi \cdot(\alpha g+h)=\alpha(\psi \cdot g)+(\psi \cdot h), \forall g, h \in \mathscr{O}_{X}^{p}$ and $\alpha \in \mathscr{O}_{X}$

b) If $M$ is generated by $\left\{h_{1}, \ldots, h_{r}\right\}$ then $\psi \cdot M$ is generated by $\left\{\psi \cdot h_{1}, \ldots, \psi \cdot h_{r}\right\}$.

Proof. It is easy to see that (b) is a straightforward consequence of (a). Now, write $g=\left(g_{1}, \ldots, g_{p}\right)$ and $h=\left(h_{1}, \ldots, h_{p}\right)$. Then, for every $z$ we have $(\psi \cdot(\alpha g+h))(z)=\sum_{i=1}^{p} \psi_{i}(z)\left(\alpha(z) g_{i}(z)+h_{i}(z)\right)=$ $\alpha(z) \sum_{i=1}^{p} \psi_{i}(z) g_{i}(z)+\sum_{i=1}^{p} \psi_{i}(z) h_{i}(z)=(\alpha(\psi \cdot g)+(\psi \cdot h))(z)$, so (a) is proved. 
Lemma 3.1.12. Suppose the submodule $M \subseteq \mathscr{O}_{X, x}^{p}$ has generic rank $k$ on each component of $X$. If $I=\left(i_{1}, \ldots, i_{k}\right)$ and $J=\left(j_{1}, \ldots, j_{k}\right)$ are indexes with $j_{1}=1$ then there exists $\psi: X \rightarrow \operatorname{Hom}\left(\mathbb{C}^{p}, \mathbb{C}\right)$ such that:

a) $\psi \cdot h=J_{I J}(h, M), \forall h \in \mathscr{O}_{X, x}^{p}$

b) $\psi \cdot M \subseteq J_{k}(M)$

Proof. Let us fix a matrix of generators of $M,[M]=\left[\begin{array}{ccc}\mid & & \mid \\ g_{1} & \ldots & g_{r} \\ \mid & & \mid\end{array}\right]$. We have $J_{I J}(h, M)=$ $\operatorname{det}[h, M]_{I J}$, where $[h, M]_{I J}=\left[\begin{array}{cccc}h_{i_{1}} & g_{i_{1}, j_{2}-1} & \ldots & g_{i_{1}, j_{k}-1} \\ \vdots & \vdots & & \vdots \\ h_{i_{k}} & g_{i_{k}, j_{2}-1} & \ldots & g_{i_{k}, j_{k}-1}\end{array}\right]$, for all $h=\left(h_{1}, \ldots, h_{p}\right)$. Let $G_{i_{l}, j_{s}-1}$ be the $(l, s)$-cofactor of $[h, M]_{I J}$, for all $l, s \in\{1, \ldots, k\}$. Notice that the $(l, 1)$-cofactors $G_{i_{l}, 0}$ does not depend of $h$. Then, $J_{I J}(h, M)=\operatorname{det}[h, M]_{I J}=\sum_{l=1}^{k} G_{i_{l}, 0} \cdot h_{i_{l}}$, for all $h$ (taking the cofactor expansion at the first column, since $\left.j_{1}-1=0\right)$. Take $\psi: X \rightarrow \operatorname{Hom}\left(\mathbb{C}^{p}, \mathbb{C}\right)$ given by $\left(\psi_{1}, \ldots, \psi_{p}\right)$ where $\psi_{i_{l}}=G_{i_{l}, 0}$, for all $l \in\{1, \ldots k\}$, and $\psi_{j}=0$, for every index $j$ off $I$. Thus, for all $h \in \mathscr{O}_{X, x}^{p}$ we get $J_{I J}(h, M)=\psi_{I} \cdot h_{I}=\psi \cdot h$. So, (a) is proved.

Now, let $g \in M$ arbitrary. Then $(g, M)=M$. By (a) we have $\psi \cdot g=J_{I, J}(g, M) \in J_{k}((g, M))=$ $J_{k}(M)$, hence $\psi \cdot M \subseteq J_{k}(M)$.

The Propositions 1.2.5 and 1.2.11 are characterizations for the integral closure of modules using integral closure of ideals. Notice the next proposition is another characterization of the same type.

Proposition 3.1.13. Let $h \in \mathscr{O}_{X, x}^{p}$ and suppose that the submodule $M \subseteq \mathscr{O}_{X, x}^{p}$ has generic rank $k$ on each component of $X$. Then, $h \in \bar{M}$ at $x$ if and only if $\psi \cdot h \in \overline{\psi \cdot M}$ at $x$, for every $\psi: X \rightarrow \operatorname{Hom}\left(\mathbb{C}^{p}, \mathbb{C}\right)$ analytic map.

Proof. $(\Longrightarrow)$ Let $\phi:(\mathbb{C}, 0) \rightarrow(X, x)$ be an arbitrary curve. Since $h \in \bar{M}$ then $h \circ \phi \in \phi^{*}(M)$, so $(\psi \circ \phi)(h \circ \phi) \in(\psi \circ \phi) \phi^{*}(M) \Rightarrow(\psi \cdot h) \circ \phi \in \phi^{*}(\psi \cdot M)$. Hence, $\psi \cdot h \in \overline{\psi \cdot M}$.

$(\Longleftarrow)$ The proof use 1.2.5 and 1.2.6.

By these results it is enough to check that $J_{I J}(h, M) \in \overline{J_{k}(M)}$, for all indexes $I$ and $J$. Write $I=\left(i_{1}, \ldots, i_{k}\right)$ and $J=\left(j_{1}, \ldots, j_{k}\right)$. Let $[M]=\left[\begin{array}{ccc}\mid & & \mid \\ g_{1} & \ldots & g_{r} \\ \mid & & \mid\end{array}\right]$ be a matrix of generators of 
$M$. Write $h=\left(h_{1}, \ldots, h_{p}\right)$. Then, $[h, M]=\left[\begin{array}{cccc}\mid & \mid & & \mid \\ h & g_{1} & \ldots & g_{r} \\ \mid & \mid & & \mid\end{array}\right]$. If $j_{1}>1$ then $J_{I J}(h, M)$ is a $k \times k$ minor taken only among the generators of $M$, hence $J_{I J}(h, M) \in J_{k}(M) \subseteq \overline{J_{k}(M)}$.

Now suppose that $j_{1}=1$. By the Lemma 3.1.12 there exists $\psi: X \rightarrow \operatorname{Hom}\left(\mathbb{C}^{p}, \mathbb{C}\right)$ such that $\psi \cdot h=J_{I, J}(h, M)$ and $\psi \cdot M \subseteq J_{k}(M)$. By the hypothesis we have $\psi \cdot h \in \overline{\psi \cdot M}$, hence $J_{I, J}(h, M)=\psi \cdot h \in \overline{\psi \cdot M} \subseteq \overline{J_{k}(M)}$, which finishes the proof.

The last proposition will be useful to prove that the 3-Lipschitz Saturation satisfies the same statement of the Propositions 3.1.3 and 3.1.8.

Definition 3.1.14. The 3-Lipschitz saturation of the submodule $M \subseteq \mathscr{O}_{X, x}^{p}$ is denoted by $M_{S_{3}}$, and is defined by

$$
M_{S_{3}}:=\left\{h \in \mathscr{O}_{X, x}^{p} \mid \psi \cdot h \in(\psi \cdot M)_{S}, \forall \psi: X \rightarrow \operatorname{Hom}\left(\mathbb{C}^{p}, \mathbb{C}\right) \text { analytic map }\right\} .
$$

In the family case, the 3-Lipschitz saturation of $\mathbf{M}$ at $\mathbf{x} \in \mathbf{X}$ relative to $Y$ is defined as above taking the Lipschitz saturation of $\psi \cdot M$ relative to $Y$.

If $\mathscr{M}$ is an $\mathscr{O}_{X}$-submodule of $\mathscr{O}_{X}^{p}$ then we can consider the sheaf of $\mathscr{O}_{X}$-modules $\mathscr{M}_{S_{3}}$ where each stalk $\left(\mathscr{M}_{S_{3}}\right)_{x}$ is $\left(\mathscr{M}_{x}\right)_{S_{3}}$.

Unless we say the opposite, we denote by $M_{S_{3}}$ the stalk of the sheaf $\mathscr{M}_{S_{3}}$ at an arbitrary point of $X$.

Remark 3.1.15. $M_{S_{3}}$ is the Lipschitz saturation of an ideal if $p=1$.

First, let us prove that $\alpha \cdot M_{S} \subseteq(\alpha \cdot M)_{S}, \forall \alpha \in \mathscr{O}_{X, x}$. In fact consider the canonical projection $\pi: S B_{M}(X) \rightarrow X$. We have a canonical projection $B_{\alpha \cdot M}(X)=B_{(\alpha)}\left(B_{M}(X)\right) \rightarrow B_{M}(X)$ which gives rise a canonical projection $\pi_{\alpha}: S B_{\alpha \cdot M}(X) \rightarrow S B_{M}(X)$ and $\pi \circ \pi_{\alpha}: S B_{\alpha \cdot M}(X) \rightarrow X$ is the canonical projection for the Lipschitz saturation of the blowup of $X$ with respect to the ideal $\alpha \cdot M$. We already know that

$$
\begin{gathered}
M_{S}=\left\{h \in \mathscr{O}_{X, x} \mid \pi^{*}(h) \in \pi^{*}(M)\right\} \text { and } \\
(\alpha \cdot M)_{S}=\left\{h \in \mathscr{O}_{X, x} \mid\left(\pi \circ \pi_{\alpha}\right)^{*}(h) \in\left(\pi \circ \pi_{\alpha}\right)^{*}(\alpha \cdot M)\right\} .
\end{gathered}
$$

Thus, given $h \in M_{S}$ we have that $\pi^{*}(h) \in \pi^{*}(M)$ which implies that $\left(\pi \circ \pi_{\alpha}\right)^{*}(\alpha h)=$ $\pi_{\alpha}^{*} \circ \pi^{*}(\alpha h)=\pi_{\alpha}^{*}\left(\pi^{*}(\alpha)\right) \cdot \pi_{\alpha}^{*}\left(\pi^{*}(h)\right) \in\left(\pi \circ \pi_{\alpha}\right)^{*}(\alpha) \cdot\left(\pi \circ \pi_{\alpha}\right)^{*}(M) \subseteq\left(\pi \circ \pi_{\alpha}\right)^{*}(\alpha \cdot M)$. Hence $\alpha h \in(\alpha M)_{S}$ and therefore $\alpha \cdot M_{S} \subseteq(\alpha \cdot M)_{S}$.

Finally, let us prove that $M_{S_{3}}=M_{S}$. Indeed, if $h \in M_{S_{3}}$, taking $\psi_{0}: X \rightarrow \operatorname{Hom}(\mathbb{C}, \mathbb{C})=\mathbb{C}$, $\psi_{0} \equiv 1$ we have that $h=\psi_{0} \cdot h \in\left(\psi_{0} \cdot M\right)_{S}=M_{S}$. Conversely, let $h \in M_{S}$. For every $\psi: X \rightarrow$ $\operatorname{Hom}(\mathbb{C}, \mathbb{C})=\mathbb{C}$ we can look to the germ $\psi \in \mathscr{O}_{X, x}$ and $\psi \cdot h \in \psi \cdot M_{S} \subseteq(\psi \cdot M)_{S}$, i.e, $\psi \cdot h \in$ $(\psi \cdot M)_{S}$. Hence, $h \in M_{S_{3}}$. 
Proposition 3.1.16. Let $\mathscr{M}$ be a sheaf of $\mathscr{O}_{X}$-submodules of $\mathscr{O}_{X}^{p}$. Then:

a) $\mathscr{M}_{S_{3}}$ is an $\mathscr{O}_{X}$-submodule of $\mathscr{O}_{X}^{p}$;

b) $M \subseteq M_{S_{3}} \subseteq \bar{M}$. In particular, $M$ is a reduction of $M_{S_{3}}$ and, if $M$ has finite colenght in $M_{S_{3}}$ then $e\left(M, M_{S_{3}}\right)=0$.

The same result holds in the family case.

Proof. Let $x \in X$ be an arbitrary point.

(a) Let $h, h^{\prime} \in \mathscr{M}_{S_{3}}$ and $\alpha \in \mathscr{O}_{X}$ at $x$, and let $\left.\psi: X \rightarrow \operatorname{Hom}\left(\mathbb{C}^{p}, \mathbb{C}\right)\right\}$ arbitrary. Then, $\psi \cdot h, \psi \cdot h^{\prime} \in(\psi \cdot M)_{S}$ at $x$, and by Lemma 3.1.11 (a) we have $\psi \cdot\left(\alpha h+h^{\prime}\right)=\alpha(\psi \cdot h)+\psi \cdot h^{\prime} \in(\psi \cdot M)_{S}$ at $x$. Therefore, $\alpha h+h^{\prime} \in \mathscr{M}_{S_{3}}$ at $x$.

(b) If $h \in M$ then $\psi \cdot h \in \psi \cdot M \subseteq(\psi \cdot M)_{S}$, so $h \in M_{S_{3}}$ and $M \subseteq M_{S_{3}}$.

Now, let $h \in M_{S_{3}}$. Then, $\psi \cdot h \in(\psi \cdot M)_{S} \subseteq \overline{\psi \cdot M}, \forall \psi: X \rightarrow \operatorname{Hom}\left(\mathbb{C}^{p}, \mathbb{C}\right)$. By Proposition 3.1.13 we conclude that $h \in \bar{M}$.

Definition 3.1.17. Consider the Setup 2.2.1. We define the infinitesimal Lipschitz conditions with respect to the 3-Lipschitz saturation of a module.

- The pair $(X, Y)$ satisfies the 3-infinitesimal Lipschitz condition $\mathbf{m}_{\mathbf{Y}}\left(3-\mathbf{i} \mathbf{L}_{\mathbf{m}_{\mathbf{Y}}}\right)$ at $\mathbf{X} \in \mathbf{X}$ if $J M(X)_{Y} \subseteq\left(m_{Y} J M_{Z}(X)\right)_{S_{3}}$ at $x$;

- The pair $(X, Y)$ satisfies the 3-infinitesimal Lipschitz condition $\boldsymbol{A}\left(3-\mathbf{i} \mathbf{L}_{\mathbf{A}}\right)$ at $\mathbf{x} \in \mathbf{X}$ if $J M(X)_{Y} \subseteq\left(J M_{Z}(X)\right)_{S_{3}}$ at $x$.

Here we consider the 3-Lipschitz saturation relative to the parameter space Y. Notice that 3-i $L_{m_{Y}}$ implies $3-i L_{A}$.

Corollary 3.1.18. Consider the Setup 2.2.1. If $x=(z, y) \in X$ with $z \neq 0$ then $3-i L_{m_{Y}}$ and 3-iL hold at $x$. Furthermore, there exists a dense Zariski open subset $U$ of $Y$ such the 3-iL $L_{A}$ holds in $(0, y)$, for all $y \in U$.

Proof. By Lemma 2.2.8 we have that $J M(X)_{Y} \subseteq m_{Y} J M_{Z}(X)$ at $x$. By Proposition 3.1.16 the inclusion $m_{Y} J M_{z}(X) \subseteq\left(m_{Y} J M_{z}(X)\right)_{S_{3}}$ holds and therefore the condition 3-iL ${m_{Y}}_{Y}$ holds at $x$. In particular, 3-i $i L_{A}$ holds at $x$.

The second statement is a consequence of the Theorem 2.2.11 and the Proposition 3.1.22. 
Proposition 3.1.19. Suppose the submodule $M \subseteq \mathscr{O}_{X, x}^{p}$ has generic rank $k$ on every component of $X$. Let $\pi_{S}: S B_{J_{k}(M)}(X) \rightarrow B_{J_{k}(M)}(X), \mathbf{p}: B_{J_{k}(M)}(X) \rightarrow X$ and $\pi=\mathbf{p} \circ \pi_{S}$ be the projections maps.

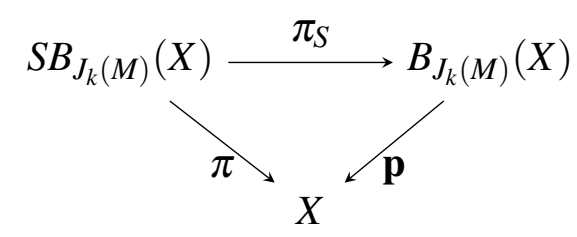

Let $h \in \mathscr{O}_{X, x}^{p}$ Then:

$$
J_{k}(h, M) \subseteq\left(J_{k}(M)\right)_{S} \text { if and only if } \pi^{*}(h) \in \pi^{*}(M)
$$

Proof. Fix a set of generators $\left\{g_{1}, \ldots, g_{r}\right\}$ of $M$. We work at $x^{\prime} \in E, E=\pi_{S}^{-1}\left(E_{B}\right), E_{B}$ the exceptional divisor of $B_{J_{k}(M)}(X)$.

Suppose first that $J_{k}(h, M) \subseteq J_{k}(M)_{S}$. Let $\pi^{*}\left(J_{I, J}(M)\right)$ be a local generator of the principal ideal $\pi^{*}\left(J_{k}(M)\right)$. Then, by Cramer's rule we can write

$$
\left(J_{I, J}(M) \circ \pi\right)\left(h_{I} \circ \pi\right)=\sum_{j \in J}\left(J_{I, j}\left(h_{I}, M_{I}\right) \circ \pi\right)\left(m_{I, j} \circ \pi\right)
$$

with $m_{j} \in M$. We claim in fact that

$$
\left(J_{I, J}(M) \circ \pi\right)(h \circ \pi)=\sum_{j \in J}\left(J_{I, j}\left(h_{I}, M_{I}\right) \circ \pi\right)\left(m_{j} \circ \pi\right)
$$

To see this pick a curve $\phi:(\mathbb{C}, 0) \rightarrow\left(B_{J_{k}(M)}(X)_{S}, x^{\prime}\right)$ and choose $\phi$ so that the rank of $\left.\pi^{*}(M)\right|_{\phi}$ is generically $k$. Since by hypothesis $h \in \bar{M}$ then $h \circ \pi \circ \phi \in \phi^{*}\left(\pi^{*}(M)\right)$. So, the element

$$
\star=h \circ \pi \circ \phi-\sum_{j \in J}\left(\frac{J_{I, j}\left(h_{I}, M_{I}\right) \circ \pi}{J_{I, J}(M) \circ \pi} \circ \phi\right)\left(m_{j} \circ \pi \circ \phi\right) \in \phi^{*}\left(\pi^{*}(M)\right)
$$

because the above quotients by hypothesis are regular functions on $S B_{J_{k}(M)}(X)$. By equation (1), the above element has 0 for the entries indexed by $I$. So, if $\star$ is not zero then $\phi^{*}\left(\pi^{*}(M)\right)$ has rank at least $k+1$, which is a contradiction. Since the images of $\phi$ fill up a Z-open set it follows that (see Lemma 3.3 of [48]) $\star$ is locally zero. Therefore,

$$
h \circ \pi=\sum_{j \in J}\left(\frac{J_{I, j}\left(h_{I}, M_{I}\right) \circ \pi}{J_{I, J}(M) \circ \pi}\right)\left(m_{j} \circ \pi\right) \in \pi^{*}(M) .
$$

Conversely, suppose that $\pi^{*}(h) \in \pi^{*}(M)$. Then we can write

$$
h \circ \pi=\sum_{j=1}^{r} \alpha_{j}\left(g_{j} \circ \pi\right)
$$


where $\alpha_{j} \in \widetilde{\mathscr{O}}_{B_{J_{k}(M)}(X), x^{\prime}}, \forall j \in\{1, \ldots, r\}$.

Let $c$ be an arbitrary generator of $J_{k}(h, M)$. Then we can write $c=\operatorname{det}[h, M]_{I J}$ for some $k$-indexes $I$ and $J$. If the $k$-index $J$ does not pick the first column of $[h, M]$, then $c$ is a $k \times k$ minor of the matrix $[M]$, hence $c \in J_{k}(M) \subset\left(J_{k}(M)\right)_{S}$ and we are done. Thus we may assume that $J$ pick the first column of $[h, M]$. Then, we can write

$$
c=\operatorname{det}\left[\begin{array}{llll}
h_{I} & \left(g_{j_{1}}\right)_{I} & \ldots & \left(g_{j_{k-1}}\right)_{I}
\end{array}\right] .
$$

Applying the pullback of the projection map we have

$$
\pi^{*}(c)=\operatorname{det}\left[h_{I} \circ \pi \quad\left(g_{j_{1}}\right)_{I} \circ \pi \quad \ldots \quad\left(g_{j_{k-1}}\right)_{I} \circ \pi\right] .
$$

By the equation (2), if we look only to the entries of the $k$-index $I$, we get

$$
h_{I} \circ \pi=\sum_{j=1}^{r} \alpha_{j}\left(\left(g_{j}\right)_{I} \circ \pi\right) .
$$

Using the linearly of the determinant in the first column, we conclude that $\pi^{*}(c)=\sum_{j=1}^{r} \alpha_{j}\left(v_{j} \circ \pi\right)$, where

$$
v_{j}:=\operatorname{det}\left[\begin{array}{llll}
\left(g_{j}\right)_{I} & \left(g_{j_{1}}\right)_{I} & \cdots & \left(g_{j_{k-1}}\right)_{I}
\end{array}\right],
$$

for all $j \in\{1, . ., r\}$. Clearly, $v_{j} \in J_{k}(M), \forall j \in\{1, \ldots, r\}$. Hence, $\pi^{*}(c) \in \pi^{*}\left(J_{k}(M)\right)$ and by the definition of the Lipschitz saturation of an ideal, we conclude that $c \in\left(J_{k}(M)\right)_{S}$. Therefore, $J_{k}(h, M) \subseteq\left(J_{k}(M)\right)_{S}$.

Corollary 3.1.20. Suppose the submodule $M \subseteq \mathscr{O}_{X, x}^{p}$ has generic rank $k$ on every component of $X$. If $J_{k}(h, M) \subseteq\left(J_{k}(M)\right)_{S}$ then there exists an open covering of $X$ such that $h$ can be written on each $U$ of the covering as an element of $M$ by using Lipschitz functions (Lipschitz with respect to $\left(z_{i}\right)$ and $\left(\frac{J_{I^{\prime}, J^{\prime}}(M)}{J_{I, J}(M)}\right)$, where $J_{I, J}(M)$ is the minor which gives the local generator on the preimage of $U$ in $\left.S B_{J_{k}(M)}(X)\right)$.

Proof. It is a straightforward consequence of the proof of the last proposition, more precisely, the equation (2) above.

Proposition 3.1.21. Suppose the submodule $M \subseteq \mathscr{O}_{X, x}^{p}$ has generic rank $k$ on each component of $X$. If $h \in M_{S_{3}}$ then $J_{k}(h, M) \subseteq\left(J_{k}(M)\right)_{S}$.

Proof. By Theorem 2.3 of [40] it is enough to prove that $\left(J_{k}(h, M)\right)_{D} \subseteq \overline{\left(J_{k}(M)\right)_{D}}$ at $(x, x)$. So, we have to prove that all the generators $\left(J_{I J}(h, M)\right)_{D}$ and $\left(0,\left(z_{i} \circ \pi_{1}-z_{i} \circ \pi_{2}\right)\left(J_{I J}(h, M)\right) \circ \pi_{2}\right)$ are in $\overline{\left(J_{k}(M)\right)_{D}}$, for all $i \in\{1, \ldots n\}$ and for all indexes $I$ and $J$. Write $I=\left(i_{1}, \ldots, i_{k}\right)$ and $J=\left(j_{1}, \ldots, j_{k}\right)$. We have two cases.

Suppose $j_{1}>1$. Then, as we have seen, $J_{I J}(h, M) \in J_{k}(M)$, so $\left(J_{I J}(h, M)\right)_{D} \in\left(J_{k}(M)\right)_{D}$, in particular, $\left(J_{I J}(h, M)\right)_{D} \in \overline{\left(J_{k}(M)\right)_{D}}$. Furthermore, since $J_{I J}(h, M) \in J_{k}(M)$, by Lemma 2.1.2 
(b) we have that

$\left(0,\left(z_{i} \circ \pi_{1}-z_{i} \circ \pi_{2}\right)\left(J_{I J}(h, M)\right) \circ \pi_{2}\right) \in\left(J_{k}(M)\right)_{D} \subseteq \overline{\left(J_{k}(M)\right)_{D}}$.

Suppose $j_{1}=1$. By the Lemma 3.1.12 there exists $\psi: X \rightarrow \operatorname{Hom}\left(\mathbb{C}^{p}, \mathbb{C}\right)$ such that $\psi \cdot h=J_{I J}(h, M)$ and $\psi \cdot M \subseteq J_{k}(M)$. Since $h$ is in the 3-Lipschitz Saturation of $M$ at $x$ then $\psi \cdot h \in(\psi \cdot M)_{S}$, which is equivalent to $(\psi \cdot h)_{D} \in \overline{(\psi \cdot M)_{D}}$. Thus, $\left(J_{I, J}(h, M)\right)_{D}=(\psi \cdot h)_{D} \in$ $\overline{(\psi \cdot M)_{D}} \subseteq \overline{\left(J_{k}(M)\right)_{D}}$. Let us prove that

$\left(0,\left(z_{i} \circ \pi_{1}-z_{i} \circ \pi_{2}\right)\left(J_{I J}(h, M) \circ \pi_{2}\right)\right) \in \overline{\left(J_{k}(M)\right)_{D}}$ by using the curve criterion. Let $\phi:(\mathbb{C}, 0) \rightarrow$ $(X \times X,(x, x))$ be an analytic curve. Write $\phi=\left(\phi_{1}, \phi_{2}\right)$. Since $\psi \cdot h \in(\psi \cdot M)_{S} \subseteq \overline{\psi \cdot M}$ then we can write $(\psi \cdot h) \circ \phi_{2}=\sum_{j} \beta_{j}\left(\left(\psi \cdot g_{j}\right) \circ \phi_{2}\right)$, with $\beta_{j} \in \mathscr{O}_{\mathbb{C}, 0}$ and $g_{j} \in M$. Thus, we get $\left(0,\left(z_{i} \circ\right.\right.$ $\left.\left.\pi_{1}-z_{i} \circ \pi_{2}\right)\left(J_{I J}(h, M) \circ \pi_{2}\right)\right) \circ \phi=\sum_{j} \beta_{j}\left(\left(0,\left(z_{i} \circ \pi_{1}-z_{i} \circ \pi_{2}\right)\left(\psi \cdot g_{j}\right) \circ \pi_{2}\right) \circ \phi\right) \in(\psi \cdot M)_{D} \circ \phi \subseteq$ $\left(J_{k}(M)\right)_{D} \circ \phi$, which finishes the proof.

Proposition 3.1.22. Let $\mathscr{M}$ be a sheaf of $\mathscr{O}_{X}$-submodules of $\mathscr{O}_{X}^{p}$. Then, $\mathscr{M}_{S_{2}} \subseteq \mathscr{M}_{S_{3}}$ in every point $x \in X$.

Proof. Let $h=\left(h_{1}, \ldots, h_{p}\right) \in \mathscr{M}_{S_{2}}$ at $x$. Then $h_{D} \in \overline{\mathscr{M}_{D}}$ at $(x, x)$. It is enough to check that $(\psi \cdot h)_{D} \in \overline{(\psi \cdot M)_{D}}$, for all $\psi: X \rightarrow \operatorname{Hom}\left(\mathbb{C}^{p}, \mathbb{C}\right)$. Let $\phi=\left(\phi_{1}, \phi_{2}\right):(\mathbb{C}, 0) \rightarrow(X \times X,(x, x))$ be an arbitrary analytic curve. Since $h_{D} \in \overline{M_{D}}$ then we can write

$$
h_{D} \circ \phi=\sum_{j} \alpha_{j} \phi^{*}\left(\left(g_{j}\right)_{D}\right)
$$

with $g_{j}=\left(g_{1 j}, \ldots, g_{p j}\right) \in M$ and $\alpha_{j} \in \mathscr{O}_{\mathbb{C}, 0}$ for all $j$. Looking to the above equation and comparing the $2 p$ coordinates we conclude that $h_{i} \circ \phi_{1}=\sum_{j} \alpha_{j}\left(g_{i j} \circ \phi_{1}\right)$ and $h_{i} \circ \phi_{2}=\sum_{j} \alpha_{j}\left(g_{i j} \circ \phi_{2}\right)$, for all $i \in\{1, \ldots, p\}$. Let $\psi_{1}, \ldots, \psi_{p}$ be the coordinates functions of $\psi$.

Thus: $(\psi \cdot h)_{D} \circ \phi=\left((\psi \cdot h) \circ \phi_{1},(\psi \cdot h) \circ \phi_{2}\right)$

$=\left(\sum_{i}\left(\psi_{i} \circ \phi_{1}\right) \cdot\left(h_{i} \circ \phi_{1}\right), \sum_{i}\left(\psi_{i} \circ \phi_{2}\right) \cdot\left(h_{i} \circ \phi_{2}\right)\right)$

$=\left(\sum_{i, j}\left(\psi_{i} \circ \phi_{1}\right) \alpha_{j}\left(g_{i j} \circ \phi_{1}\right), \sum_{i, j}\left(\psi_{i} \circ \phi_{2}\right) \alpha_{j}\left(g_{i j} \circ \phi_{2}\right)\right)$

$=\sum_{j} \alpha_{j}\left(\sum_{i}\left(\psi_{i} \circ \phi_{1}\right) \cdot\left(g_{i j} \circ \phi_{1}\right), \sum_{i}\left(\psi_{i} \circ \phi_{2}\right) \cdot\left(g_{i j} \circ \phi_{2}\right)\right)$

$=\sum_{j} \alpha_{j}\left(\left(\psi \cdot g_{j}\right) \circ \phi_{1},\left(\psi \cdot g_{j}\right) \circ \phi_{2}\right)$

$=\sum_{j} \alpha_{j} \underbrace{\left(\left(\psi \cdot g_{j}\right)_{D} \circ \phi\right)}_{\in(\psi \cdot M)_{D} \circ \phi} \in(\psi \cdot M)_{D} \circ \phi$, and the proof is done.

Definition 3.1.23. Suppose that $M \subseteq \mathscr{O}_{X, x}^{p}$ is an $\mathscr{O}_{X, x}$-submodule of generic rank $k$ on each component of $X$. The 4-Lipschitz saturation of $M$ is denoted by $M_{S_{4}}$, and is defined by

$$
M_{S_{4}}:=\left\{h \in \mathscr{O}_{X, x}^{p} \mid J_{k}(h, M) \subseteq\left(J_{k}(M)\right)_{S}\right\}
$$

In the family case, the 4-Lipschitz saturation of $\mathrm{M}$ at $\mathbf{x} \in \mathbf{X}$ relative to $\mathrm{Y}$ is defined as above taking the Lipschitz saturation of $J_{k}(M)$ relative to $Y$. 
If $\mathscr{M}$ is an $\mathscr{O}_{X}$-submodule of $\mathscr{O}_{X}^{p}$ then we can consider the sheaf of $\mathscr{O}_{X}$-modules $\mathscr{M}_{S_{4}}$ where each $\operatorname{stalk}\left(\mathscr{M}_{S_{4}}\right)_{x}$ is $\left(\mathscr{M}_{x}\right)_{S_{4}}$.

Unless we say the opposite, we denote by $M_{S_{4}}$ the stalk of the sheaf $\mathscr{M}_{S_{4}}$ at an arbitrary point of $X$.

Remark 3.1.24. $M_{S_{4}}$ is the Lipschitz saturation of an ideal if $p=1$. In fact, since $p=1$ then the generic rank of $M$ is 0 or 1 . If the generic rank is 0 then $M=M_{S_{4}}=M_{S}=(0)$. Suppose the generic rank is 1. Then: $h \in M_{S_{4}} \Leftrightarrow J_{1}(h, M) \subseteq\left(J_{1}(M)\right)_{S} \Leftrightarrow(h, M) \subseteq M_{S} \Leftrightarrow h \in M_{S}$.

The next proposition allows us to see $\mathscr{M}_{S_{4}}$ as a sheaf of $\mathscr{O}_{X}$-submodules of $\mathscr{O}_{X}^{p}$.

Proposition 3.1.25. Let $\mathscr{M}$ be a sheaf of $\mathscr{O}_{X}$-submodules of $\mathscr{O}_{X}^{p}$ of generic rank $k$ on each component of $X$. Then:

a) $\mathscr{M}_{S_{4}}$ is an $\mathscr{O}_{X}$-submodule of $\mathscr{O}_{X}^{p}$;

b) $M \subseteq M_{S_{4}} \subseteq \bar{M}$. In particular, $M$ is a reduction of $M_{S_{4}}$ and, if $M$ has finite colenght in $M_{S_{4}}$ then $e\left(M, M_{S_{4}}\right)=0$.

The same result holds in the family case.

Proof. (a) Let $g, h \in \mathscr{M}_{S_{4}}$ at $x \in X$ and $\alpha \in \mathscr{O}_{X, x}$. By the basic properties of determinants we have that

$$
J_{k}(\alpha g+h, M) \subseteq \alpha J_{k}(g, M)+J_{k}(h, M) \subseteq\left(J_{k}(M)\right)_{S}
$$

Hence, $\alpha g+h \in M_{S_{4}}$ at $x$.

(b) Since $J_{k}(M) \subseteq\left(J_{k}(M)\right)_{S}$ then $M \subseteq M_{S_{4}}$. Now, let $h \in M_{S_{4}}$. Thus, $J_{k}(h, M) \subseteq\left(J_{k}(M)\right)_{S} \subseteq$ $\overline{J_{k}(M)}$ which implies that $h \in \bar{M}$. Therefore, the inclusion $M_{S_{4}} \subseteq \bar{M}$ is proved.

Definition 3.1.26. Consider the Setup 2.2.1. We define the infinitesimal Lipschitz conditions with respect to the 4-Lipschitz saturation of a module.

- The pair $(X, Y)$ satisfies the 4-infinitesimal Lipschitz condition $\mathbf{m}_{\mathbf{Y}}\left(\right.$ (4-iL $\left.\mathbf{m}_{\mathbf{Y}}\right)$ at $\mathbf{x} \in \mathbf{X}$ if $J M(X)_{Y} \subseteq\left(m_{Y} J M_{Z}(X)\right)_{S_{4}}$ at $x ;$

- The pair $(X, Y)$ satisfies the 4-infinitesimal Lipschitz condition $A\left(4-\mathbf{i L}_{\mathbf{A}}\right)$ at $\mathbf{x} \in \mathbf{X}$ if $J M(X)_{Y} \subseteq\left(J M_{z}(X)\right)_{S_{4}}$ at $x$.

Here we consider the 4-Lipschitz saturation relative to the parameter space Y. Notice that 4-i $L_{m_{Y}}$ implies $4-i L_{A}$.

Corollary 3.1.27. Consider the Setup 2.2.1. If $x=(z, y) \in X$ with $z \neq 0$ then 4-i $L_{m_{Y}}$ and 4-i $L_{A}$ hold at $x$. Furthermore, there exists a dense Zariski open subset $U$ of $Y$ such the 4-i $L_{A}$ holds in $(0, y)$, for all $y \in U$. 
Proof. By Lemma 2.2.8 we have that $J M(X)_{Y} \subseteq m_{Y} J M_{z}(X)$ at $x$. By Proposition 3.1.25 the inclusion $m_{Y} J M_{z}(X) \subseteq\left(m_{Y} J M_{z}(X)\right)_{S_{4}}$ holds and therefore the condition 4-iL $L_{m_{Y}}$ holds at $x$. In particular, 4-i $L_{A}$ holds at $x$.

The second statement is a consequence of the Theorem 2.2.11, Propositions 3.1.21 and 3.1.22.

Notice that we can rephrase the Proposition 3.1.21 by saying that $\mathscr{M}_{S_{3}} \subseteq \mathscr{M}_{S_{4}}$ at every point $x \in X$.

Example 3.1.28. Consider $X=\mathbb{C}$ with coordinate $t$ and $p=2$. Consider the $\mathscr{O}_{\mathbb{C}, 0 \text {-submodule }}$ $M \subseteq \mathscr{O}_{\mathbb{C}, 0}^{2}$ generated by $\{(t, 0)\}$. Let $h:=(0, t) \in \mathscr{O}_{\mathbb{C}, 0}^{2}$. Notice that the generic rank of $M$ is $1,[M]=\left[\begin{array}{l}t \\ 0\end{array}\right]$ and $[h, M]=\left[\begin{array}{ll}0 & t \\ t & 0\end{array}\right]$. Thus, $J_{1}(h, M)=(t)$ and $J_{1}(M)=(t)$. In particular, $J_{1}(h, M) \subseteq J_{1}(M) \subseteq\left(J_{1}(M)\right)_{S}$ which implies that $h \in M_{S_{4}}$.

Now consider $\psi: \mathbb{C} \rightarrow \operatorname{Hom}\left(\mathbb{C}^{2}, \mathbb{C}\right)$ given by $\psi(t):=(t, 1)$. Then $\psi \cdot M=\left(t^{2}\right)$ and $\psi \cdot h=t$. Since $t \notin \overline{\left(t^{2}\right)}$ then $\psi \cdot h \notin \overline{\psi \cdot M}$, in particular, $\psi \cdot h \notin(\psi \cdot M)_{S}$. Hence, $h \notin M_{S_{3}}$ and we conclude that $M_{S_{3}} \subsetneq M_{S_{4}}$.

In [39] Gaffney gave a geometric interpretation of the infinitesimal Lipschitz condition A, presenting vector fields defined in a open covering of $B_{J_{z}(G)}(X)$, where $X$ is a hypersurface defined by $G$. Here we extend this interpretation for the jacobian module case.

Consider $X$ a family of analytic varieties as on the setup 2.2.1. Let $s$ be the generic rank of $M:=J M_{z}(X)$. Let $g$ be the number of generators of $J_{S}(M)$, i.e, $g=\#\{(I, J) \mid I$ and $J$ are $s$-indexes $\}$. Let $T_{I J}$ be the homogeneous coordinates of $\mathbb{P}^{g-1}$ and $M_{I J}$ be the generators of $J_{S}(M),(I, J)$ varying on the set of all pairs of $s$-indexes. Consider the diagram

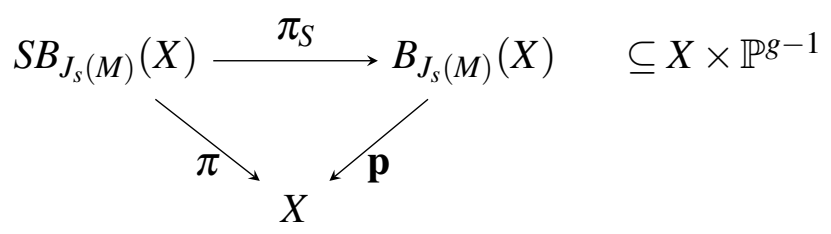

as in the Proposition 3.1.19. Let $V_{I J}$ be the dense Zariski open subset of $\mathbb{P}^{g-1}$ defined by $T_{I J} \neq 0$ and let $U_{I J}:=B_{J_{S}(M)}(X) \cap\left(X \times V_{I J}\right)$. Clearly the collection $\left\{U_{I J}\right\}_{(I, J)}$ is an open covering of $B_{J_{S}(M)}(X)$. At each point of $U_{I J}, M_{I J} \circ \pi$ is a local generator of the principal ideal sheaf $\pi^{*}\left(J_{S}(M)\right)$.

Suppose that $J M(X)_{Y} \subseteq\left(J M_{Z}(X)\right)_{S_{t}}$ for some $t \in\{2,3,4\}$. By Propositions 3.1.21 and 3.1.22 we have $J M(X)_{Y} \subseteq\left(J M_{z}(X)\right)_{S_{4}}$. By the proof of the Proposition 3.1.19, for each $\ell \in$ $\{1, \ldots, k\}$ we can write

$$
\frac{\partial F}{\partial y_{\ell}} \circ \pi=\sum_{j \in J} \frac{M_{I J, j, \ell} \circ \pi}{M_{I J} \circ \pi}\left(\frac{\partial F}{\partial z_{j}} \circ \pi\right)
$$


where $M_{I J, j, \ell}$ is the determinant of the matrix obtained by replacing the $j^{\text {th }}$ column of $M_{I J}$ by $\left(\frac{\partial F}{\partial y_{\ell}}\right)_{I}$, for all $j \in J$.

Thus, for each pair of $s$-indexes $(I, J)$, we have $s . k$ vector fields tangent to $X$

$$
\vec{v}_{I J, j, \ell}:=\frac{\partial}{\partial y_{\ell}}-\frac{M_{I J, j, \ell}}{M_{I J}} \frac{\partial}{\partial z_{j}}
$$

defined on $\pi\left(U_{I J}\right)$. Since $\frac{M_{I J, j, \ell} \circ \pi}{M_{I J} \circ \pi}$ is Lipschitz, for all $j \in J$ and $\ell \in\{1, \ldots, k\}$ then $\vec{v}_{I J, j, \ell}$ is a Lipschitz vector field relative to $Y$.

\subsection{The generic equivalence among the Lipschitz satura- tions}

Now we look for conditions ensuring the above notions of Lipschitz saturations are generically equivalent. The next theorem gives us a such condition.

Theorem 3.2.1. Let $M$ be an $\mathscr{O}_{X, x}$-submodule of $\mathscr{O}_{X, x}^{p}$ with generic rank $k$ on each component of $X$ and $h \in \mathscr{O}_{X, x}^{p}$. Suppose that there exists an ideal I of $\mathscr{O}_{X \times X,(x, x)}^{2 p}$ such that

$$
\begin{gathered}
I . J_{2}\left(\left(J_{k}(M)\right)_{D}\right) \subseteq \overline{J_{2 k}\left(M_{D}\right)} \\
J_{2 k}\left(h_{D}, M_{D}\right) \subseteq \overline{I . J_{2}\left(\left(J_{k}(h, M)\right)_{D}\right)} .
\end{gathered}
$$

Then, the following condition are equivalent:

(a) $h \in M_{S_{2}}$;

(b) $h \in M_{S_{3}}$;

(c) $h \in M_{S_{4}}$.

Proof. $(a) \Longrightarrow(b)$ Follows from the Proposition 3.1.22.

$(b) \Longrightarrow(c)$ Follows from the Proposition 3.1.21.

$(c) \Longrightarrow(a)$ Suppose that $J_{k}(h, M) \subseteq\left(J_{k}(M)\right)_{S}$. Then, $\left(J_{k}(h, M)\right)_{D} \subseteq \overline{\left(J_{k}(M)\right)_{D}}$ and so

$$
\overline{\left(J_{k}(h, M)\right)_{D}}=\overline{\left(J_{k}(M)\right)_{D}}
$$

Let us prove that $J_{2 k}\left(h_{D}, M_{D}\right) \subseteq \overline{J_{2 k}\left(M_{D}\right)}$. In fact, let $\phi:(\mathbb{C}, 0) \rightarrow(X \times X,(x, x))$ be an arbitrary analytic curve. Since $\overline{\left(J_{k}(h, M)\right)_{D}}=\overline{\left(J_{k}(M)\right)_{D}}$ then $\phi^{*}\left(\left(J_{k}(h, M)\right)_{D}\right)=\phi^{*}\left(\left(J_{k}(M)\right)_{D}\right)$. Using the inclusions of the hypothesis and the curve criterion for the integral closure of modules we have $\phi^{*}\left(J_{2 k}\left(h_{D}, M_{D}\right)\right) \subseteq \phi^{*}\left(I . J_{2}\left(\left(J_{k}(h, M)\right)_{D}\right)\right)=\phi^{*}(I) \cdot \phi^{*}\left(J_{2}\left(\left(J_{k}(h, M)\right)_{D}\right)\right)$ 
$=\phi^{*}(I) . J_{2}\left(\phi^{*}\left(\left(J_{k}(h, M)\right)_{D}\right)\right)=\phi^{*}(I) . J_{2}\left(\phi^{*}\left(\left(J_{k}(M)\right)_{D}\right)\right)=\phi^{*}(I) \cdot \phi^{*}\left(J_{2}\left(\left(J_{k}(M)\right)_{D}\right)\right)$

$=\phi^{*}\left(I . J_{2}\left(\left(J_{k}(M)\right)_{D}\right)\right) \subseteq \phi^{*}\left(J_{2 k}\left(M_{D}\right)\right)$. Hence, the desired inclusion is proved.

By Theorem 2.1.17 we have that $M_{D}$ has generic rank $2 k$ on each component of $(X \times X,(x, x))$. Therefore, by Corollary 1.2 .6 we conclude that $h_{D} \in \overline{M_{D}}$.

Corollary 3.2.2. Let $M \subseteq \mathscr{O}_{X, x}^{p}$ be an $\mathscr{O}_{X, x}$-submodule of generic rank $k$ on each component of $X$. Suppose that there exists an ideal I of $\mathscr{O}_{X \times X,(x, x)}^{2 p}$ such that

$$
\begin{gathered}
I . J_{2}\left(\left(J_{k}(M)\right)_{D}\right) \subseteq \overline{J_{2 k}\left(M_{D}\right)} \\
J_{2 k}\left(h_{D}, M_{D}\right) \subseteq \overline{I . J_{2}\left(\left(J_{k}(h, M)\right)_{D}\right)}, \forall h \in \bar{M} .
\end{gathered}
$$

Then

$$
M_{S_{2}}=M_{S_{3}}=M_{S_{4}}
$$

In order to use the above criterion, we prove some useful lemmas that allow us to get the desired equivalence.

Notation: For each object $A$, we denote $A=A \circ \pi_{1}$ and $A^{\prime}=A \circ \pi_{2}$. For $k$-indexes $I, J$, we denote by $M_{I J}$ the submatrix of $[M]$ defined by these $k$-indexes.

Lemma 3.2.3. Let $M \subseteq \mathscr{O}_{X, x}^{p}$ be a submodule and $k \in \mathbb{N}$. Then

$$
\left(z_{t_{1}}-z_{t_{1}}^{\prime}\right) \ldots\left(z_{t_{k}}-z_{t_{k}}^{\prime}\right) \operatorname{det}\left(M_{I J}\right) \operatorname{det}\left(M_{K L}^{\prime}\right) \in J_{2 k}\left(M_{D}\right) a t(x, x)
$$

for every $t_{1}, \ldots, t_{k} \in\{1, \ldots, n\}$ and $k$-indexes $I, J, K, L$.

Proof. Write $M_{K L}=\left(g_{r s}\right)$. The $2 k \times 2 k$ matrix

$$
\left[\begin{array}{c|ccc}
M_{I J} & 0_{k \times k} \\
-------- & ------------ \\
& \left(z_{t_{1}}-z_{t_{1}}^{\prime}\right) g_{11}^{\prime} & \ldots & \left(z_{t_{k}}-z_{t_{k}}^{\prime}\right) g_{1 k}^{\prime} \\
M_{I J}^{\prime} & \vdots & & \vdots \\
& \left(z_{t_{1}}-z_{t_{1}}^{\prime}\right) g_{k 1}^{\prime} & \ldots & \left(z_{t_{k}}-z_{t_{k}}^{\prime}\right) g_{k k}^{\prime}
\end{array}\right]
$$

is a submatrix of $\left[M_{D}\right]$. So, its determinant, which is $\left(z_{t_{1}}-z_{t_{1}}^{\prime}\right) \ldots\left(z_{t_{k}}-z_{t_{k}}^{\prime}\right) \operatorname{det}\left(M_{I J}\right) \operatorname{det}\left(M_{K L}^{\prime}\right)$, belogns to $J_{2 k}\left(M_{D}\right)$.

The next result gives us the first condition of the Theorem 3.2.1 in terms of the ideal coming from the diagonal.

Lemma 3.2.4. Let $M \subseteq \mathscr{O}_{X, x}^{p}$ be a submodule and $k \in \mathbb{N}$.

a) $I_{\Delta}^{k} J_{2}\left(\left(J_{k}(M)\right)_{D}\right) \subseteq J_{2 k}\left(M_{D}\right)$ at $(x, x)$;

b) If $J_{k}(M)$ is principal then $I_{\Delta}^{k-1} J_{2}\left(\left(J_{k}(M)\right)_{D}\right) \subseteq J_{2 k}\left(M_{D}\right)$ at $(x, x)$. 
Proof. (a) We have that

$$
\left[\left(J_{k}(M)\right)_{D}\right]=\left[\begin{array}{ccc}
\operatorname{det}\left(M_{I J}\right) & \ldots & 0 \\
\operatorname{det}\left(M_{I J}^{\prime}\right) & \ldots & \left(z_{i}-z_{i}^{\prime}\right) \operatorname{det}\left(M_{K L}^{\prime}\right)
\end{array}\right]
$$

varying the $k$-indexes $I, J, K, L$ and $i \in\{1, \ldots, n\}$.

Thus, the desired inclusion is a straightforward consequence of the previous lemma.

(b) Since $J_{k}(M)$ is principal then there exist $k$-indexes $I, J$ such that $g=\operatorname{det}\left(M_{I J}\right)$ and $J_{k}(M)$ is generated by $\{g\}$. Thus we can write

$$
\left[\left(J_{k}(M)\right)_{D}\right]=\left[\begin{array}{cc}
g & 0 \\
g^{\prime} & \left(z_{i}-z_{i}^{\prime}\right) g^{\prime}
\end{array}\right]
$$

varying $i \in\{1, \ldots n\}$.

Thus, in this case $J_{2}\left(\left(J_{k}(M)\right)_{D}\right)$ is generated by $\left\{g \cdot g^{\prime}\left(z_{i}-z_{i}^{\prime}\right) \mid i \in\{1, \ldots, n\}\right\}$. So, by previous lemma we have that

$$
\left[\left(z_{t_{1}}-z_{t_{1}}^{\prime}\right) \ldots\left(z_{t_{k-1}}-z_{t_{k-1}}^{\prime}\right)\right] \cdot\left[g \cdot g^{\prime}\left(z_{i}-z_{i}^{\prime}\right)\right] \in J_{2 k}\left(M_{D}\right)
$$

for all $t_{1}, \ldots, t_{k-1}, i \in\{1, \ldots, n\}$.

Therefore, $I_{\Delta}^{k-1} J_{2}\left(\left(J_{k}(M)\right)_{D}\right) \subseteq J_{2 k}\left(M_{D}\right)$ at $(x, x)$.

Now, we start to get conditions in order to obtain the second condition of the Theorem 3.2.1.

Lemma 3.2.5. Let $M$ be an $\mathscr{O}_{X, x}$-submodule of $\mathscr{O}_{X, x}^{p}$ and $k \in \mathbb{N}$. Denote by $[\tilde{M}]$ the matrix such that

$$
\left[M_{D}\right]=\left[\begin{array}{cc}
{[M]} & 0 \\
{[M]^{\prime}} & {[\tilde{M}]}
\end{array}\right] .
$$

Let $\mathscr{J}_{2 k}\left(M_{D}\right)$ be the subideal of $J_{2 k}\left(M_{D}\right)$ generated by

$$
\left\{\operatorname{det}\left(M_{I J}\right) \operatorname{det}\left(\tilde{M}_{K L}\right) \mid I, J, K, L \text { are } k \text {-indexes }\right\} .
$$

Then,

$$
\mathscr{J}_{2 k}\left(M_{D}\right) \subseteq I_{\Delta}^{k-1} J_{2}\left(\left(J_{k}(M)\right)_{D}\right) \text { at }(x, x)
$$

Proof. It suffices to prove that each generator of $\mathscr{J}_{2 k}\left(M_{D}\right)$ belongs to $I_{\Delta}^{k-1} J_{2}\left(\left(J_{k}(M)\right)_{D}\right)$.

The columns of $\tilde{M}_{K L}$ are columns of $[M]^{\prime}$ possibly in a different order with terms of type $z_{i}-z_{i}^{\prime}$ multiplied on each column. If the $k$-indexes $K, L$ pick repeated columns then $\operatorname{det}\left(\tilde{M}_{K L}\right)=0$ and we are done. If this does not occur then

$$
\operatorname{det}\left(\tilde{M}_{K L}\right)=\left(z_{i_{1}}-z_{i_{1}}^{\prime}\right) \ldots\left(z_{i_{k}}-z_{i_{k}}^{\prime}\right)\left( \pm \operatorname{det}\left(M_{K^{\prime} L^{\prime}}^{\prime}\right)\right)
$$


for some reorganization $k$-indexes $K^{\prime}, L^{\prime}$, where $i_{1}, \ldots, i_{k} \in\{1, \ldots, n\}$ are the indexes which $z_{i_{1}}-z_{i_{1}}^{\prime}, \ldots, z_{i_{k}}-z_{i_{k}}^{\prime}$ appear on each of the $k$ columns of $\tilde{M}_{K L}$.

Since $\pm\left(z_{i_{1}}-z_{i_{1}}^{\prime}\right) \ldots\left(z_{i_{k-1}}-z_{i_{k-1}}^{\prime}\right) \in I_{\Delta}^{k-1}$ and $\operatorname{det}\left(M_{I J}\right)\left(z_{i_{k}}-z_{i_{k}}^{\prime}\right) \operatorname{det}\left(M_{K L}^{\prime}\right) \in J_{2}\left(\left(J_{k}(M)\right)_{D}\right)$ then $\operatorname{det}\left(M_{I J}\right) \operatorname{det}\left(\tilde{M}_{K L}\right) \in I_{\Delta}^{k-1} J_{2}\left(\left(J_{k}(M)\right)_{D}\right)$, which finishes the proof.

The next result ensures that any free submodule satisfies the second condition of the Theorem 3.2.1 for a suitable power of the ideal coming from the diagonal.

Lemma 3.2.6. Let $M$ be a free $\mathscr{O}_{X, x}$-submodule of $\mathscr{O}_{X, x}^{p}$ of rank $k$. Then

$$
J_{2 k}\left(M_{D}\right) \subseteq I_{\Delta}^{k-1} J_{2}\left(\left(J_{k}(M)\right)_{D}\right) a t(x, x)
$$

Proof. We have that

$$
\left[M_{D}\right]=\left[\begin{array}{c|c}
{[M]_{p \times k}} & 0 \\
------ & ------- \\
{[M]_{p \times k}^{\prime}} & \left(z_{i}-z_{i}^{\prime}\right)[M]^{\prime}
\end{array}\right]
$$

varying $i \in\{1, \ldots, n\}$.

Let $d \in J_{2 k}\left(M_{D}\right)$ at $(x, x)$ be an arbitrary generator. Then $d=\operatorname{det} N$ where $N$ is a $2 k \times 2 k$ submatrix of $\left[M_{D}\right]$.

(i) Suppose first that there are $k+t$ columns of $N$ taken on the part

$$
\left[\begin{array}{c}
0 \\
\left(z_{i}-z_{i}^{\prime}\right)[M]^{\prime}
\end{array}\right]
$$

of $\left[M_{D}\right]$, with $1 \leq t \leq k$. Then we can write

$$
\begin{aligned}
N= & {\left[\begin{array}{c|c}
\left(M_{I J}\right)_{k \times(k-t)} & 0_{k \times(k+t)} \\
------- & ------- \\
\left(M_{I J}\right)_{k \times(k-t)}^{\prime} & (\tilde{M})_{K L}
\end{array}\right] } \\
& =\left[\begin{array}{cc|c}
M_{I J} & 0_{k \times t} & 0_{k \times k} \\
--- & --- & ------ \\
M_{I J}^{\prime} & * & * *
\end{array}\right]
\end{aligned}
$$

for some matrices $*$ and $* *$, where $* *$ is a square matrix of size $k \times k$. So in this case we have that $d=\operatorname{det} N=\operatorname{det}\left[\begin{array}{ll}M_{I J} & 0_{k \times t}\end{array}\right] \cdot \operatorname{det}(* *)=0 \in I_{\Delta}^{k-1} J_{2}\left(\left(J_{k}(M)\right)_{D}\right)$.

(ii) Now suppose that we have exactly $k$ columns of $N$ taken on the part

$$
\left[\begin{array}{c}
0 \\
\left(z_{i}-z_{i}^{\prime}\right)[M]^{\prime}
\end{array}\right]
$$


of $\left[M_{D}\right]$. Then we can write

$$
N=\left[\begin{array}{c|c}
M_{I J} & 0_{k \times k} \\
------ & ------ \\
M_{I J}^{\prime} & \tilde{M}_{K L}
\end{array}\right]
$$

Thus, $d=\operatorname{det}\left(M_{I J}\right) \operatorname{det}\left(\tilde{M}_{K L}\right) \in \mathscr{J}_{2 k}\left(M_{D}\right)$ and by previous lemma we conclude that $d \in I_{\Delta}^{k-1} J_{2}\left(\left(J_{k}(M)\right)_{D}\right)$.

As a consequence of the previous lemma, we get another lemma which states that the second condition of the Theorem 3.2.1 holds in a dense Zariski open subset of $X$ in a sheaf of submodules of $\mathscr{O}_{X}^{p}$.

Lemma 3.2.7. Let $\mathscr{M}$ be a sheaf of $\mathscr{O}_{X}$-submodules of $\mathscr{O}_{X}^{p}$ of generic rank $k$ on each component of $X$. Then there exists a dense Zariski open subset $U$ of $X$ such that $U \cap V$ is a dense Zariski open subset of $V, \forall V$ component of $X, J_{k}(\mathscr{M})$ is principal at $x$ and

$$
J_{2 k}\left(\mathscr{M}_{D}\right) \subseteq I_{\Delta}^{k-1} J_{2}\left(\left(J_{k}(\mathscr{M})\right)_{D}\right) \text { at }(x, x),
$$

for all $x \in U$.

Proof. Consider $[\mathscr{M}]$ a matrix of generators of $\mathscr{M}$. Using the Cramer's rule, we can choose $k$ $\mathscr{O}_{X}$-linear independents columns of $[\mathscr{M}]$ such that these columns generates $\mathscr{M}$ in a such dense Zariski open subset $U$ of $X$. Let $\mathscr{M}_{k}$ be the $\mathscr{O}_{X}$-submodule of $\mathscr{O}_{X}^{p}$ generated by the columns chosen above. Thus, given $x \in U$, we have that $\mathscr{M}_{x}=\left(\mathscr{M}_{k}\right)_{x}$ is a free $\mathscr{O}_{X, x}$-submodule of $\mathscr{O}_{X, x}^{p}$ of rank $k$ and the desired inclusion is a consequence of the previous lemma. The ideal $J_{k}(\mathscr{M})=J_{k}\left(\mathscr{M}_{k}\right)$ is principal at $x$, because $\left[\mathscr{M}_{k}\right]$ is a square $k \times k$ matrix.

Lemma 3.2.8. Let $M$ be an $\mathscr{O}_{X, x}$-submodule of $\mathscr{O}_{X, x}^{p}$ of generic rank $k$ on each component of $X$, where $\mathscr{O}_{X, x}$ is a reduced ring. If $h \in \bar{M}$ then $(h, M)$ also has generic rank $k$ on each component of $X$.

Proof. Let $V$ be an arbitrary component of $(X, x)$ and let $t$ be the generic rank of $(h, M)$ at $V$. Since $V$ is irreducible then there exists a prime ideal $I_{x}$ of $\mathscr{O}_{X, x}$ such that $\mathscr{O}_{V, x}=\frac{\mathscr{O}_{X, x}}{I_{x}}$. Since $I_{x}$ is a prime ideal (hence radical ideal) then $\mathscr{O}_{V, x}$ is a domain (hence reduced ring).

Since $M \subseteq(h, M)$ then $t \geq k$. Suppose by contradiction that $t>k$. Then $J_{t}(M)=(0)$, once $M$ has generic rank $k$. Since $h \in \bar{M}$ then (see [41]) $J_{t}(h, M) \subseteq \overline{J_{t}(M)}=\overline{(0)} \subseteq \sqrt{(0)}=(0)$ at the component $V$. Hence, $J_{t}(h, M)=(0)$ at $V$, contradiction. Therefore, $t=k$.

The next theorem gives us the equivalence desired. 
Theorem 3.2.9. Let $\mathscr{M}$ be a sheaf of $\mathscr{O}_{X}$-submodules of $\mathscr{O}_{X}^{p}$ of generic rank $k$ on each component of $X$, and suppose that $X$ has reduced structure. Then there exists a dense Zariski open subset $U$ of $X$ such that $U \cap V$ is a dense Zariski open subset of $V$, for all $V$ component of $X$ and

$$
\mathscr{M}_{S_{2}}=\mathscr{M}_{S_{3}}=\mathscr{M}_{S_{4}}
$$

along $U$.

In the case when $k=p$ is the maximal rank, the same conclusion holds even if $X$ does not have reduced structure.

Proof. Since $X \subseteq \mathbb{C}^{n}$ is an analytic complex variety then $\mathscr{O}_{X}$ is a noetherian sheaf of rings, hence $\mathscr{O}_{X}^{p}$ is a noetherian sheaf of $\mathscr{O}_{X}$-modules. Since $\mathscr{M}_{S_{4}}$ is a sheaf of $\mathscr{O}_{X}$-modules then $\mathscr{M}_{S_{4}}$ is finitely generated by global sections $h_{1}, \ldots, h_{r}$. Since $h_{i} \in \overline{\mathscr{M}}, \forall i \in\{1, \ldots, r\}$ then by previous lemma $\left(h_{i}, \mathscr{M}\right)$ also has generic rank $k$ on each component of $X, \forall i \in\{1, \ldots, r\}$. By Lemma 3.2.7, for each $i \in\{1, \ldots, r\}$ there exists a dense Zariski open subset $U_{i}$ of $X$ such that $U_{i} \cap V$ is a dense Zariski open subset of $V$, for all $V$ component of $X$, and

$$
J_{2 k}\left(\left(h_{i}\right)_{D}, \mathscr{M}_{D}\right) \subseteq I_{\Delta}^{k-1} J_{2}\left(\left(J_{k}\left(h_{i}, \mathscr{M}\right)\right)_{D}\right) \text { at }(x, x),
$$

$\forall x \in U_{i}$

Also by Lemma 3.2.7 there exists a dense Zariski open subset $U_{0}$ of $X$ such that $U_{0} \cap V$ is a dense Zariski open subset of $V$, for all component $V$ of $X$, and $J_{k}(M)$ is principal at $x, \forall x \in U_{0}$. From the Lemma 3.2.4(b), we conclude that

$$
I_{\Delta}^{k-1} J_{2}\left(\left(J_{k}(\mathscr{M})\right)_{D}\right) \subseteq J_{2 k}\left(\mathscr{M}_{D}\right) \text { at }(x, x)
$$

$\forall x \in U_{0}$.

Take $U:=\bigcap_{j=0}^{r} U_{j}$. Then $U$ is a dense Zariski open subset of $X$ and $U \cap V$ is a dense Zariski open subset of $V$, for all $V$ component of $X$.

We already know that $\mathscr{M}_{S_{2}} \subseteq \mathscr{M}_{S_{3}} \subseteq \mathscr{M}_{S_{4}}$ at any point of $X$, in particular, along $U$.

Let us prove that $\mathscr{M}_{S_{4}} \subseteq \mathscr{M}_{S_{2}}$ along $U$. In fact, let $x \in U$. Given an arbitrary $i \in\{1, \ldots, r\}$, since $x \in U_{0}$ and $x \in U_{i}$ then

$$
\begin{gathered}
I_{\Delta}^{k-1} J_{2}\left(\left(J_{k}(\mathscr{M})\right)_{D}\right) \subseteq J_{2 k}\left(\mathscr{M}_{D}\right) \text { at }(x, x) \\
\text { and } \\
J_{2 k}\left(\left(h_{i}\right)_{D}, \mathscr{M}_{D}\right) \subseteq I_{\Delta}^{k-1} J_{2}\left(\left(J_{k}\left(h_{i}, \mathscr{M}\right)\right)_{D}\right) \text { at }(x, x) .
\end{gathered}
$$

Since $h_{i} \in \mathscr{M}_{S_{4}}$ at $x$ then by Theorem 3.2.1 we have that $h_{i} \in \mathscr{M}_{S_{2}}$ at $x, \forall i \in\{1, \ldots, r\}$. Since $\mathscr{M}_{S_{4}}$ is generated by $h_{1}, \ldots, h_{r}$ at $x$, then $\mathscr{M}_{S_{4}} \subseteq \mathscr{M}_{S_{2}}$ at $x$, and the theorem is proved. 


\subsection{Geometric applications}

The next result gives us conditions envolving the double and the integral closure of modules so that we can construct Lipschitz vector fields.

Proposition 3.3.1. Let $X \subseteq \mathbb{C}^{n}$ be an analytic curve. Let $\mathscr{M}$ be an $\mathscr{O}_{X}$-submodule of $\mathscr{O}_{X}^{p}$ of generic rank $k$, with $k+1$ generators. Let $\mathscr{M}_{k}$ be a reduction of $\mathscr{M}$ generated by the first $k$ columns of $[\mathscr{M}]$, whose rank is $k$. Let $h \in \mathscr{O}_{X}^{p}$. Let $c(h) \in \mathscr{O}_{X}^{p}$ be defined by the solution of the equation

$$
\left[\mathscr{M}_{k}\right] \cdot c(h)=h
$$

in a dense Zariski open subset $U$ of $X$ (using the Cramer's Rule). Let $x \in U, M=\mathscr{M}_{x}, M_{k}=$ $\left(\mathscr{M}_{k}\right)_{x}$ and $m=m_{X, x}$. If $h \in \overline{m M}$ and $h_{D} \in \overline{\left(M_{k}\right)_{D}}$ at $(x, x)$ then the vector field $c(h)$ is Lipschitz, i.e, $c(h)-c(h)^{\prime} \in \overline{I_{\Delta} \mathscr{O}_{X \times X}^{p}}$ at $(x, x)$.

Proof. Let us use the curve criterion. Let $\phi: \mathbb{C}, 0 \rightarrow X \times X,(x, x)$ be a curve, with coordinates $\phi_{1}, \phi_{2}$.

First suppose $\phi_{1}(t) \equiv 0$. In this case we have

$$
\left[\begin{array}{l}
h \circ \phi_{1} \\
h \circ \phi_{2}
\end{array}\right]=\left[\begin{array}{c}
0 \\
h \circ \phi_{2}
\end{array}\right]=\left[\begin{array}{c}
0 \\
{\left[M_{k}\right]^{\prime} \cdot c(h)}
\end{array}\right] \quad \bmod \phi_{2}^{*}\left(m M_{k}^{\prime}\right) \mathscr{O}_{\mathbb{C}, 0}^{p}
$$

because $h \circ \phi_{2} \in \phi_{2}^{*}\left(m M_{k}^{\prime}\right)$. Let $m_{1}, \ldots, m_{k}$ be the columns of $\left[M_{k}\right]$. Suppose that $z_{i} \circ \phi_{2}$ is a generator of $\phi_{2}^{*}(m)$. For each $j \in\{1, \ldots, k\}$ look to

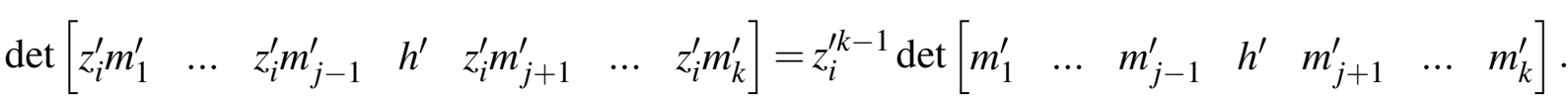

Then,

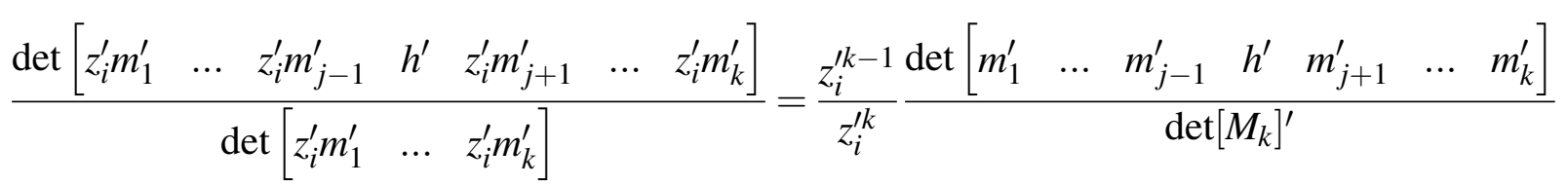

$=\frac{1}{z_{i}^{\prime}} c(h)_{j}^{\prime}$ which is analytic along $\phi_{2}, \forall j \in\{1, . . k\}$. Thus, $c(h)^{\prime} \in z_{i}^{\prime} \mathscr{O}_{X}^{p}$ along $\phi_{2}$ and since $c(h)=0$ along $\phi_{1}$ then $\phi^{*}\left(c(h)-c(h)^{\prime}\right) \in \phi^{*}\left(I_{\Delta} \mathscr{O}_{X \times X}^{p}\right)$.

The case where $\phi_{2} \equiv 0$ is analogous.

Now assume that $\phi_{1}, \phi_{2} \neq 0$. Since $\left(h_{D}\right) \in \overline{\left(M_{k}\right)_{D}}$ then

$$
\left(0,\left[M_{k}\right]^{\prime} \cdot\left(c(h)^{\prime}-c(h)\right) \in \overline{\left(M_{k}\right)_{D}} .\right.
$$

Since $M_{k} \circ \phi_{1}$ has rank $k$ generically, then $M_{k}^{\prime}\left(c(h)^{\prime}-c(h)\right) \in M_{k}^{\prime} I_{\Delta} \mathscr{O}_{X \times X}^{p}$ at $(x, x)$ along $\phi$, so $c(h)^{\prime}-c(h) \in I_{\Delta} \mathscr{O}_{X \times X}^{p}$ along $\phi$ at $(x, x)$, which finishes the proof. 


\section{Bi-Lipschitz equisingularity in an ICIS family of irreducible curves}

Let $X \subseteq \mathbb{C} \times \mathbb{C}^{n}$ be an ICIS family of irreducible curves. Assume that we have a normalization $F: \mathbb{C} \times \mathbb{C} \rightarrow X \subseteq \mathbb{C} \times \mathbb{C}^{n}$ which is a homeomorphism, and suppose that the family $X$ is Whitney equisingular. Let $p$ be the multiplicity of $X$ and assume we can write

$$
F(t, s)=\left(t, F_{1}(t, s), \ldots, F_{n-1}(t, s), s^{p}\right)
$$

where for each parameter $t$ the order of

$$
s \mapsto F_{i}(t, s)
$$

is greater than $p, \forall i \in\{1, \ldots, n-1\}$.

We already know that if $\frac{\partial F_{1}}{\partial t}, \ldots, \frac{\partial F_{n}}{\partial t}$ are Lipschitz functions on $X$ then $X$ is bi-Lipschitz equisingular.

Let $G: \mathbb{C} \times \mathbb{C}^{n} \rightarrow \mathbb{C}^{q}$ be an analytic map that defines $X$, i.e, $X=G^{-1}(0)$. Consider the jacobian module $J M(X)=J M(G)$. Here we consider the double and the 2-Lipschitz saturation relative to the parameter space $Y=\mathbb{C} \times 0 \equiv \mathbb{C}$.

The following proposition characterizes the above condition in terms of the 2-Lipschitz saturation (or the double), which gives us an infinitesimal condition for the bi-Lipschitz equisingularity.

Proposition 3.3.2. With the above notations, the functions $\frac{\partial F_{1}}{\partial t}, \ldots, \frac{\partial F_{n}}{\partial t}$ are Lispchitz if and only if

$$
\left(\frac{\partial G}{\partial t}\right)_{D} \in \overline{\left(D G_{n-1}\right)_{D}}
$$

where $D G_{n-1}$ denotes the submodule of $J M_{z}(X)$ generated by the first $n-1$ partial derivatives of $G$ with respect to the $z$ coordinates.

In particular, if $J M(X)_{Y} \subseteq\left(J M_{z}(X)\right)_{S_{2}}$ then $X$ is bi-Lipschitz equisingular.

Proof. Since $G \circ F \equiv 0$ then

$$
0=\left[\frac{\partial(G \circ F)}{\partial t}\right]=\left[\begin{array}{lllll}
\frac{\partial G}{\partial t} \circ F & \frac{\partial G}{\partial z_{1}} \circ F & \ldots & \frac{\partial G}{\partial z_{n-1}} \circ F & \frac{\partial G}{\partial z_{n}} \circ F
\end{array}\right] \cdot\left[\begin{array}{c}
1 \\
\frac{\partial F_{1}}{\partial t} \\
\vdots \\
\frac{\partial F_{n-1}}{\partial t} \\
0
\end{array}\right]
$$

which implies that

$$
\frac{\partial G}{\partial t} \circ F=-\left[D G_{n-1} \circ F\right] \cdot\left[\begin{array}{c}
\frac{\partial F_{1}}{\partial t} \\
\vdots \\
\frac{\partial F_{n-1}}{\partial t}
\end{array}\right]
$$


We also have that

$$
0=\frac{\partial(G \circ F)}{\partial s}=[J M(G) \circ F] \cdot\left[\begin{array}{c}
\frac{\partial F_{1}}{\partial s} \\
\vdots \\
\frac{\partial F_{n-1}}{\partial s} \\
p s^{p-1}
\end{array}\right] .
$$

Hence,

$$
\frac{\partial G}{\partial z_{n}} \circ F=\left[D G_{n-1} \circ F\right] \cdot\left[\begin{array}{c}
-\frac{\dot{x}_{1}(s)}{p s^{p-1}} \\
\vdots \\
-\frac{\dot{x}_{n-1}(s)}{p s^{p-1}}
\end{array}\right]
$$

Thus, for each parameter $t$ we have that $J M_{z}(X)_{\left.\right|_{X_{t}}} \subseteq \overline{D G_{n-\left.1\right|_{X_{t}}}}$. Since $X$ is a Whitney equisingular family of curves then the multiplicity of the members of the family does not depend on the parameter and we can apply the Principle of Specialization (Theorem 1.8 of [48]) to conclude that $J M_{z}(X) \subseteq \overline{D G_{n-1}}$, i.e, $D G_{n-1}$ is a reduction of $J M_{z}(X)$.

Let $c\left(\frac{\partial G}{\partial t}\right)$ be the vector field associated to the Cramer's rule in order to solve the equation

$$
\left[\frac{\partial G}{\partial t}\right]=\left[D G_{n-1}\right] \cdot \xi
$$

Since $\frac{\partial G}{\partial t} \circ F=\left[D G_{n-1} \circ F\right] \cdot\left[\begin{array}{c}-\frac{\partial F_{1}}{\partial t} \\ \vdots \\ -\frac{\partial F_{n-1}}{\partial t}\end{array}\right]$ then $c\left(\frac{\partial G}{\partial t}\right) \circ F=\left[\begin{array}{c}-\frac{\partial F_{1}}{\partial t} \\ \vdots \\ -\frac{\partial F_{n-1}}{\partial t}\end{array}\right]$.

Therefore, $\frac{\partial F_{1}}{\partial t}, \ldots, \frac{\partial F_{n}}{\partial t}$ are Lipschitz functions if and only if $c\left(\frac{\partial G}{\partial t}\right) \circ F$ is Lipschitz which is equivalent to $\left(\frac{\partial G}{\partial t}\right)_{D} \in \overline{\left(D G_{n-1}\right)_{D}}$. 

CHAPTER

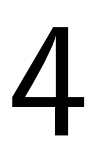

\section{BI-LIPSCHITZ EQUISINGULARITY OF DETERMINANTAL SURFACES}

In [70] Mostowski shows that every analytic variety has a stratified vector field which is Lipschitz, however, this vector field is not canonical from the variety. In [40], Gaffney got conditions so that a family of irreducible curves has a canonical vector field which is Lipschitz, namely

$$
\frac{\partial}{\partial y}+\sum_{i} \frac{\partial \widetilde{f}}{\partial y} \cdot \frac{\partial}{\partial z_{i}}
$$

where $\widetilde{F}: \mathbb{C} \times \mathbb{C} \longrightarrow \mathbb{C} \times \mathbb{C}^{n}$ given by $\widetilde{F}(y, t)=(y, \tilde{f}(y, t))$ defines the family of curves. In this case, the main condition is the Segre number $s_{2}\left(I_{D}(\tilde{F})\right)$ to be independent of the parameter $y$. Gaffney showed that the last condition is equivalent to the multiplicity of the pair $e\left(I_{D}(\tilde{F}), I_{\Delta}\right)$ to be independent of the parameter $y$, where $I_{\Delta}$ is the ideal which defines the diagonal on $\mathbb{C} \times \mathbb{C}$ and $I_{D}(\tilde{F})$ is the ideal generated by the doubles of the components of $\tilde{F}$. For more about the Segre numbers see [46].

In this chapter we get conditions so that the above vector field is Lipschitz on the context of determinantal varieties. It following the approach of Pereira and Ruas [89], we see that for the special case of determinantal surfaces, there are deformations $\tilde{f}: \mathbb{C}^{q} \longrightarrow \operatorname{Hom}\left(\mathbb{C}^{n}, \mathbb{C}^{m}\right)$ such that the above vector field always is Lipschitz. 


\subsection{Simple isolated Cohen-Macaulay of codimension 2 singularities in $\mathbb{C}^{4}$ and $\mathbb{C}^{5}$}

In [37], Frübis-Krüger and Neumer determine a complete classification of simple CohenMacaulay codimension 2 singularities. This classification was obtained up to isomorphism of germs.

A singularity is called simple if it can only deform into finitely many different isomorphism classes.

The Cohen-Macaulay singularities in codimension 2 are particularly important. One special reason is that not all of them are complete intersection, however, the theorem of HibertBruch provides a powerful tool in order to describe these singularities and their deformations.

First they classified the possible candidates of 1 -jets with 4 or more variables.

Theorem 4.1.1. ([37] Lemma 3.2) Let $M$ be a $3 \times 2$ matrix with entries in the maximal ideal of $\mathbb{C}\left\{x_{1}, \ldots, x_{m}\right\}$. Then, $j_{1} M$ is contact-equivalent to one of the jets in the following tables or is contact-equivalent to a 1-jet containing only 3 or less variables.

\begin{tabular}{|c|c|c|c|c|c|}
\hline & 6 variables & & 5 variables & & 4 variables \\
\hline \multirow[t]{6}{*}{$J^{(6,1)}$} & $\left(\begin{array}{lll}x & y & v \\
z & w & u\end{array}\right)$ & $J^{(5,1)}$ & $\left(\begin{array}{lll}z & y & v \\
z & w & x\end{array}\right)$ & $J^{(4,1)}$ & $\left(\begin{array}{lll}w & y & x \\
z & w & y\end{array}\right)$ \\
\hline & & $J^{(5,2)}$ & $\left(\begin{array}{ccc}x & y & v \\
z & w & 0\end{array}\right)$ & $\boldsymbol{J}^{(4,2)}$ & $\left(\begin{array}{lll}w & y & x \\
z & w & 0\end{array}\right)$ \\
\hline & & & & $J^{(4,3)}$ & $\left(\begin{array}{lll}0 & y & x\end{array}\right)$ \\
\hline & & & & $J^{(4,4)}$ & $\left(\begin{array}{lll}x & y & z \\
& & 0\end{array}\right)$ \\
\hline & & & & $J^{(4,5)}$ & $\left(\begin{array}{lll}x & y & 0\end{array}\right)$ \\
\hline & & & & $J^{(4,6)}$ & $\left(\begin{array}{lll}x & y & z \\
w & 0 & 0\end{array}\right)$ \\
\hline
\end{tabular}

Table 1 - Possible candidates of 1-jets with 4 or more variables

In [37] the authors have seen that 1 -jets containing only 2 or fewer variables cannot be simple in dimension 4 , so we have to consider only the 1 -jets with 3 and 4 variables. We denote $\tau$ as the Tjurina number. 
Theorem 4.1.2. ([37] Theorem 3.3) The list of simple isolated Cohen-Macaulay in codimension 2 singularities in $\left(\mathbb{C}^{4}, 0\right)$ are the following ones:

Name of triple

Jet-type Type Presentation Matrix $\tau$ point in [100]

\begin{tabular}{|c|c|c|c|c|c|}
\hline$J^{(4,1)}$ & $\Lambda_{1,1}$ & $\left(\begin{array}{ccc}w & y & x \\
z & w & y\end{array}\right)$ & & 2 & $A_{0,0,0}$ \\
\hline $\boldsymbol{J}^{(4,2)}$ & $\Lambda_{k, 1}$ & $\left(\begin{array}{ccc}w & y & x \\
z & w & y^{k}\end{array}\right)$ & $k \geq 2$ & $k+1$ & $A_{0,0, k-1}$ \\
\hline$J^{(4,3)}$ & $\Lambda_{k, l}$ & $\left(\begin{array}{ccc}w^{l} & y & x \\
z & w & y^{k}\end{array}\right)$ & $k \geq l \geq 2$ & $k+l$ & $A_{0, l-1, k-1}$ \\
\hline$J^{(4,4)}$ & & $\left(\begin{array}{ccc}z & y & x \\
x & w & y^{2}+z^{k}\end{array}\right)$ & $\begin{array}{l}k \geq 2 \\
k \geq 1\end{array}$ & $\begin{array}{l}k+3 \\
2 k+4\end{array}$ & $\begin{array}{l}C_{k+1,0} \\
B_{2 k+2,0}\end{array}$ \\
\hline & & $\left(\begin{array}{ccc}z & y & x \\
x & w & y z+y^{k}\end{array}\right)$ & $k \geq 3$ & $\begin{array}{l}2 k+1 \\
7\end{array}$ & $\begin{array}{l}B_{2 k-1,0} \\
D_{0} \\
F_{0}\end{array}$ \\
\hline
\end{tabular}

$J^{(3,1)} \quad\left(\begin{array}{ccc}z & y+w^{l} & w^{m} \\ w^{k} & y & x\end{array}\right) \quad k, l, m \geq 2 \quad k+l+m-1 \quad A_{k-1, l-1, m-1}$

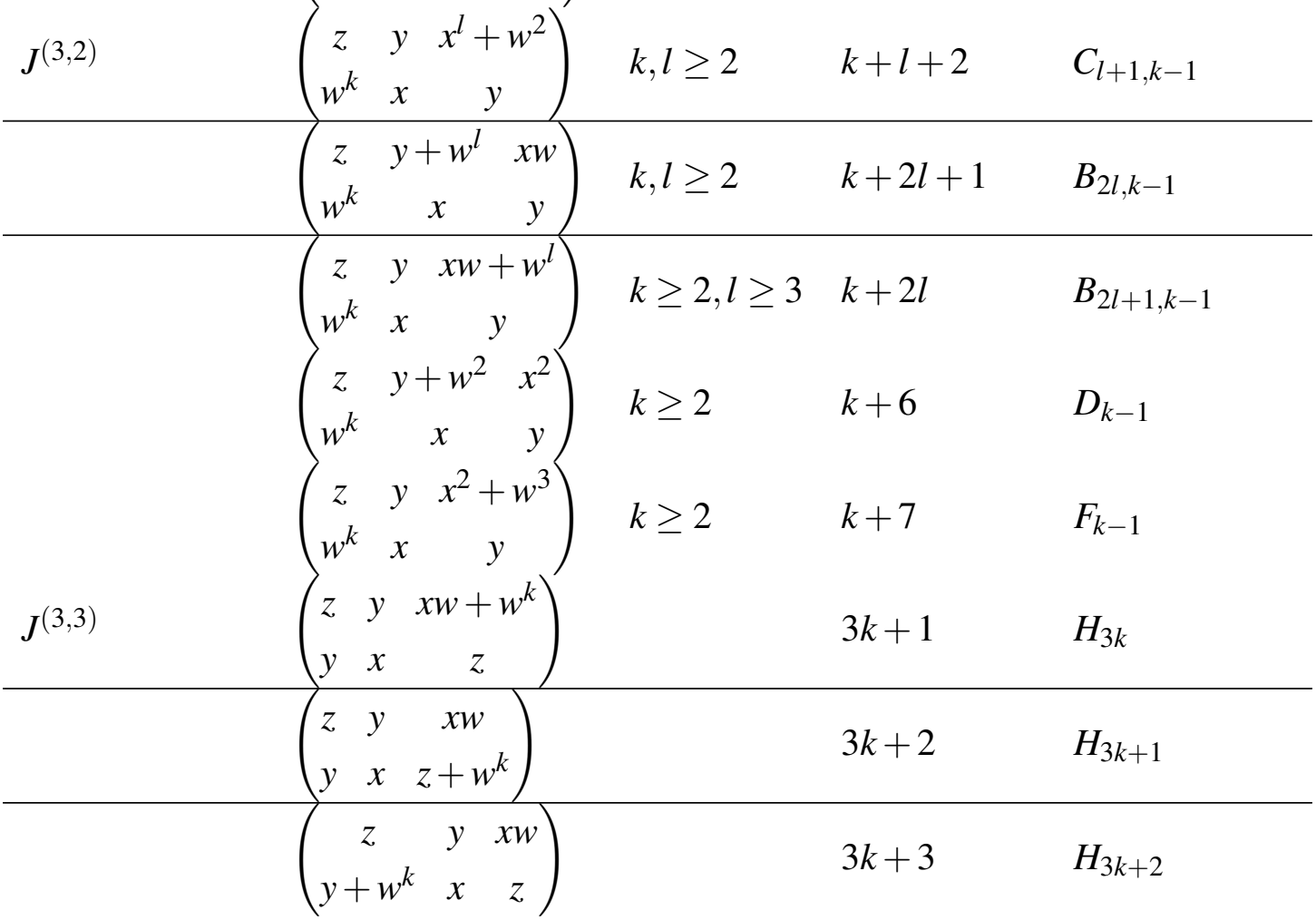




$$
\left(\begin{array}{ccc}
z & y & w^{2} \\
y & x & z+x^{2}
\end{array}\right)
$$

Table 2 - Simple isolated Cohen-Macaulay in codimension 2 singularities in $\left(\mathbb{C}^{4}, 0\right)$

In the case in $\left(\mathbb{C}^{5}, 0\right)$, in [37] the authors realized that we only need to consider matrices whose 1 -jet involves at least 4 variables. The methods are basically the same as in the previous case, with one exception: For the case $J^{(5,2)}$, the problem of classification and of finding adjacencies can be reduced to the corresponding problem for plane curve singularities and deformations with sections thereof.

\begin{tabular}{|c|c|c|c|c|}
\hline Jet-type & Type & Presentation Matrix & & $\tau$ \\
\hline$J^{(5,1)}$ & $A_{0}^{\#}$ & $\left(\begin{array}{lll}x & y & z \\
w & v & x\end{array}\right)$ & & 1 \\
\hline$J^{(5,2)}$ & $A_{k}^{\#}$ & $\left(\begin{array}{ccc}x & y & z \\
w & v & x^{k+1}+y^{2}\end{array}\right.$ & $k \geq 1$ & $k+2$ \\
\hline & $D_{k}^{\sharp}$ & $\left(\begin{array}{ccc}x & y & z \\
w & v & x y^{2}+x^{k-1}\end{array}\right)$ & $k \geq 4$ & $k+2$ \\
\hline & $E_{6}^{\sharp}$ & $\left(\begin{array}{ccc}x & y & z \\
w & v & x^{3}+v^{4}\end{array}\right.$ & & 8 \\
\hline & $E_{7}^{\sharp}$ & $\left(\begin{array}{ccc}x & y & z \\
w & v & x^{3}+x y^{3}\end{array}\right)$ & & 9 \\
\hline & $E_{8}^{\sharp}$ & $\left(\begin{array}{ccc}x & y & z \\
w & v & x^{3}+y^{5}\end{array}\right.$ & & 10 \\
\hline$J^{(4,1)}$ & $\Pi_{k}$ & $\left(\begin{array}{ccc}w & y & x \\
z & w & y+v^{k}\end{array}\right)$ & $k \geq 2$ & $2 k-1$ \\
\hline$J^{(4,2)}$ & & $\left(\begin{array}{ccc}w & y & x \\
z & w & y^{k}+v^{2} \\
w & y & x \\
z & w & y v+v^{k}\end{array}\right)$ & $k \geq 2$ & $\begin{array}{l}k+2 \\
2 k\end{array}$ \\
\hline & & 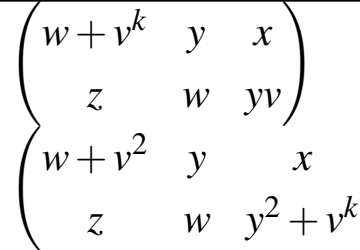 & & $\begin{array}{l}2 k+1 \\
k+3\end{array}$ \\
\hline
\end{tabular}

Theorem 4.1.3. ([37] Theorem 3.5) The simple isolated Cohen-Macaulay in codimension 2 singularities in $\left(\mathbb{C}^{5}, 0\right)$ are the following ones: 


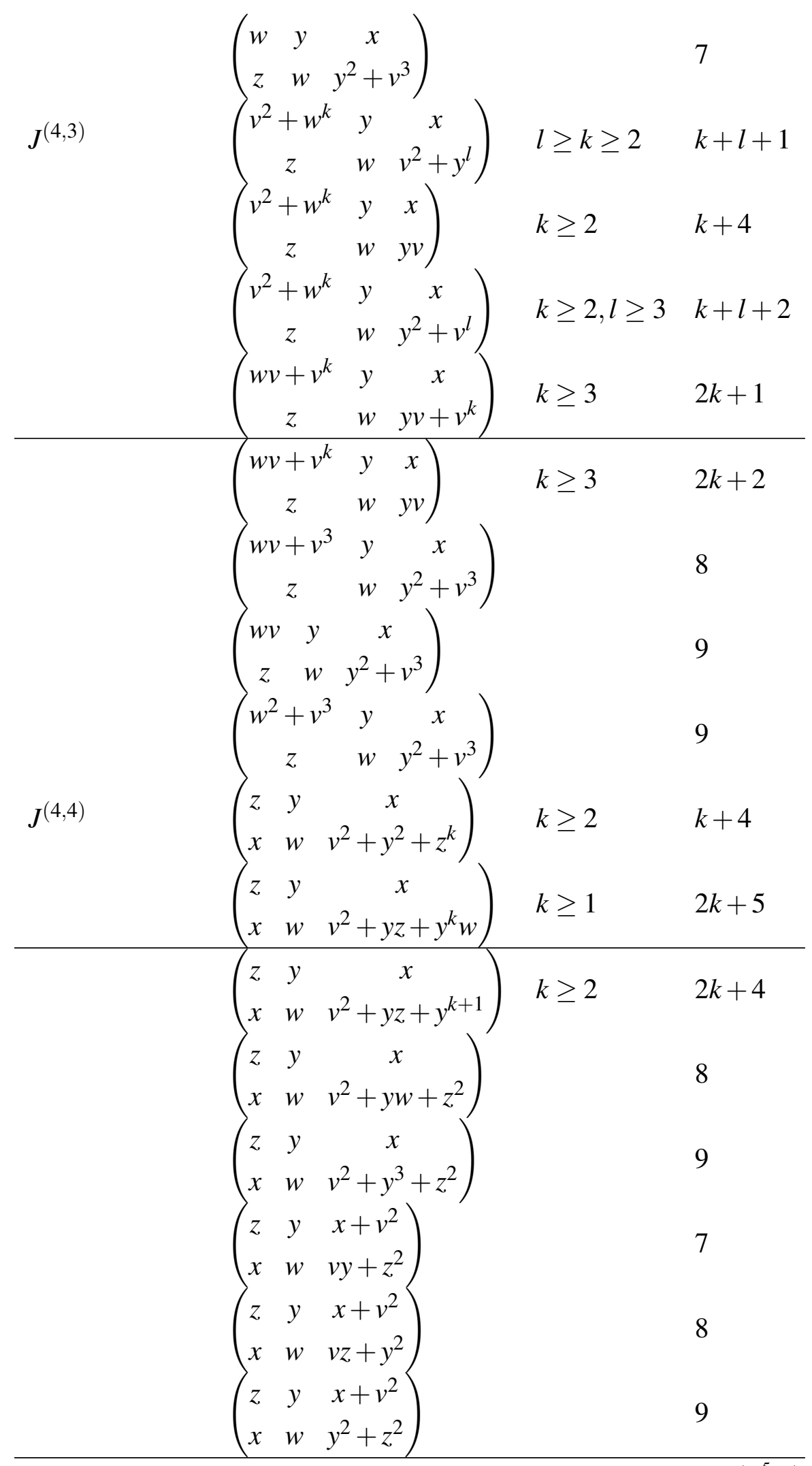

Table 3 - Simple isolated Cohen-Macaulay in codimension 2 singularities in $\left(\mathbb{C}^{5}, 0\right)$ 


\subsection{The bi-Lipschitz equisingularity on determinantal va- rieties}

Let us fix some notations.

We work with one parameter deformations and unfoldings. The parameter space is denoted by $Y=\mathbb{C} \equiv \mathbb{C} \times 0$.

Let $h \in \mathscr{O}_{\mathbb{C}^{N}}$. The $(\mathbf{1}, \mathbf{- 1})$-double of $h$ is the element denoted by $I_{D}(h) \in \mathscr{O}_{\mathbb{C}^{2 N}}$ defined by the equation

$$
I_{D}(h)\left(z, z^{\prime}\right):=h(z)-h\left(z^{\prime}\right)
$$

If $h=\left(h_{1}, \ldots, h_{r}\right)$ is a map, with $h_{i} \in \mathscr{O}_{\mathbb{C}^{N}}, \forall i \in\{1, \ldots, r\}$, then we define $I_{D}(h)$ as the the ideal of $\mathscr{O}_{\mathbb{C}^{2 N}}$ generated by $\left\{I_{D}\left(h_{1}\right), \ldots, I_{D}\left(h_{r}\right)\right\}$.

The above notions was defined by Gaffney in [39].

Now we get a relation between the integral closure of the double and the canonical vector field induced by a one parameter unfolding to be Lipschitz.

Let $\tilde{F}: \mathbb{C} \times \mathbb{C}^{q} \longrightarrow \mathbb{C} \times \mathbb{C}^{n}$ be an analytic map, which is a homeomorphism onto its image, and such that we can write $\tilde{F}(y, x)=(y, \tilde{f}(y, x))$, with $\tilde{f}(y, x)=\left(\tilde{f}_{1}(y, x), \ldots, \tilde{f}_{n}(y, x)\right)$. Let us denote by

$$
\frac{\partial}{\partial y}+\sum_{j=1}^{n} \frac{\partial \widetilde{f}}{\partial y} \cdot \frac{\partial}{\partial z_{j}}
$$

the vector field $v: \tilde{F}\left(\mathbb{C} \times \mathbb{C}^{q}\right) \longrightarrow \mathbb{C} \times \mathbb{C}^{n}$ given by

$$
v(y, z)=\left(1, \frac{\partial \tilde{f}_{1}}{\partial y}\left(\tilde{F}^{-1}(y, z)\right), \ldots, \frac{\partial \tilde{f}_{n}}{\partial y}\left(\tilde{F}^{-1}(y, z)\right)\right) .
$$

Proposition 4.2.1. The vector field $\frac{\partial}{\partial y}+\sum_{j=1}^{n} \frac{\partial \tilde{f}}{\partial y} \cdot \frac{\partial}{\partial z_{j}}$ is Lipschitz if and only if

$$
I_{D}\left(\frac{\partial \tilde{F}}{\partial y}\right) \subsetneq \overline{I_{D}(\tilde{F})}
$$

Proof. Since we are working in a finite dimensional $\mathbb{C}$-vector space then all the norms are equivalent. To simplify the argument, we use the notation $\|$.$\| for the maximum norm on \mathbb{C} \times \mathbb{C}^{q}$ and $\mathbb{C} \times \mathbb{C}^{n}$, i.e, $\left\|\left(x_{1}, \ldots, x_{n+1}\right)\right\|=\max _{i=1}^{n+1}\left\{\left\|x_{i}\right\|\right\}$.

Suppose that the canonical vector field is Lipschitz. By hypothesis there exists a constant $c>0$ such that

$$
\left\|v(y, z)-v\left(y^{\prime}, z^{\prime}\right)\right\| \leq c\left\|(y, z)-\left(y^{\prime}, z^{\prime}\right)\right\|
$$

$\forall(y, z),\left(y^{\prime}, z^{\prime}\right) \in U$, where $U$ is a non-empty open subset of $\tilde{F}\left(\mathbb{C} \times \mathbb{C}^{q}\right)$. 
Thus, given $(y, x),\left(y^{\prime}, x^{\prime}\right) \in \tilde{F}^{-1}(U)$, applying the above inequality on these points, we get

$$
\left\|\left(\frac{\partial \tilde{f}_{j}}{\partial y}\right)_{D}\left(y, x, y^{\prime}, x^{\prime}\right)\right\| \leq c\left\|\tilde{F}(y, x)-\tilde{F}\left(y^{\prime}, x^{\prime}\right)\right\|
$$

for all $j \in\{1, \ldots n\}$. By Theorem 1.1.23 each generator of $I_{D}\left(\frac{\partial \tilde{F}}{\partial y}\right)$ belongs to $\overline{I_{D}(\tilde{F})}$.

Now suppose that $I_{D}\left(\frac{\partial \tilde{F}}{\partial y}\right) \subsetneq \overline{I_{D}(\tilde{F})}$. Using the hypothesis and again the Lejeune-Teissier theorem, for each $j \in\{1, \ldots n\}$ there exists a constant $c_{j}>0$ and an open subset $U_{j} \subsetneq \mathbb{C} \times \mathbb{C}^{q}$ such that

$$
\begin{gathered}
\left\|\left(\frac{\partial \tilde{f}_{j}}{\partial y}\right)_{D}\left(y, x, y^{\prime}, x^{\prime}\right)\right\| \leq c_{j}\left\|\tilde{F}(y, x)-\tilde{F}\left(y^{\prime}, x^{\prime}\right)\right\| \\
\forall(y, x),\left(y^{\prime}, x^{\prime}\right) \in U_{j} \text {. Take } U:=\bigcap_{j=1}^{n} U_{j}, c:=\max \left\{c_{j}\right\}_{j=1}^{n} \text { and } V:=\tilde{F}(U), \text { which is an }
\end{gathered}
$$
open subset of $\tilde{F}\left(\mathbb{C} \times \mathbb{C}^{q}\right)$, since $\tilde{F}$ is a homeomorphism onto its image. Hence, it is easy to see that

$$
\left\|v(y, z)-v\left(y^{\prime}, z^{\prime}\right)\right\| \leq c\left\|(y, z)-\left(y^{\prime}, z^{\prime}\right)\right\|
$$

$\forall(y, z),\left(y^{\prime}, z^{\prime}\right) \in V$

Therefore, the vector field $\frac{\partial}{\partial y}+\sum_{j=1}^{n} \frac{\partial \tilde{f}_{j}}{\partial y} \cdot \frac{\partial}{\partial z_{j}}$ is Lipschitz .

Now, we have an application for a special case of determinantal surfaces.

Proposition 4.2.2. Suppose that $\tilde{F}: \mathbb{C} \times \mathbb{C}^{q} \longrightarrow \mathbb{C} \times H o m\left(\mathbb{C}^{m}, \mathbb{C}^{n}\right)$ is an analytic map and a homeomorphism onto its image, and suppose we can write

$$
\tilde{F}(y, x)=(y, F(x)+y \theta(x))
$$

a) The vector field $\frac{\partial}{\partial y}+\sum_{j} \frac{\partial \widetilde{f}}{\partial y} \cdot \frac{\partial}{\partial z_{j}}$ is Lipschitz if and only if

$$
I_{D}(\theta) \subsetneq \overline{I_{D}(\tilde{F})}
$$

b) If $\theta$ is constant then the vector field $\frac{\partial}{\partial y}+\sum_{j} \frac{\partial \tilde{f}}{\partial y} \cdot \frac{\partial}{\partial z_{j}}$ is Lipschitz.

Proof. (a) It is a straightforward consequence of the last proposition because $\frac{\partial \tilde{f}}{\partial y}=\theta$.

(b) Since $\theta$ is constant then the doubles of the components of $\theta$ are all zero, so $I_{D}(\theta)$ is the zero ideal, which ensures the inclusion $I_{D}(\theta) \subsetneq \overline{I_{D}(\tilde{F})}$.

Remark 4.2.3. In [2] and [76], the authors consider a one parameter deformation with a constant $\theta$. As showed above, for all these deformations the canonical vector field is Lispchitz.

In Example 4.2.4 we see a case where the deformation does not come from a constant $\theta$, and the canonical vector field remains Lipschitz. In Example 4.2.5 we have another deformation that does not come from a constant $\theta$ where the canonical vector field is not Lipschitz. 
As we have seen before, the canonical vector field is naturally associated to the 1unfolding of the variety. However, its behaviour for the Lipschitz equisingularity is not the same. This behaviour depends on the type of the normal form, as we will see later.

The space of the first order deformations $T_{X}^{1}$ can be identified with $\frac{\operatorname{Mat}_{(n, p)}\left(\mathscr{O}_{r}\right)}{\mathscr{T} \mathscr{G} F}$, where $\mathscr{T} \mathscr{G} F$ is the extended $\mathscr{G}$-tangent space of the matrix $F$ (Lemma 2.3, [37]). Hence we can treat the base of the semi-universal deformation using matrix representation and $F$ is $\mathscr{G}$-finitely determined if and only if $T_{X}^{1}$ is a finite dimensional module. From now on, the element $\theta$ is taken

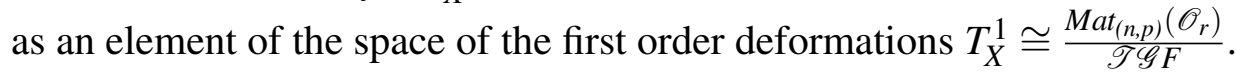

Example 4.2.4. Consider

$$
F=\left(\begin{array}{ccc}
w^{l} & y & x \\
z & w & y^{k}
\end{array}\right)
$$

with $l, k \geq 2$, which is one of the normal forms obtained in Table 2. Consider the matrix of deformation

$$
\theta=\left(\begin{array}{ccc}
\sum_{i=0}^{l-1} w^{i} & 0 & 0 \\
0 & 0 & \sum_{j=0}^{k-1} y^{j}
\end{array}\right)
$$

and $\tilde{F}(u, x, y, z, w)=\left(u, F(x, y, z, w)+u \theta(x, y, z, w)\right.$. Notice that $\theta \in \frac{\operatorname{Mat}_{(2,3)}\left(\mathscr{O}_{4}\right)}{T \mathscr{G} F}$ and $I_{D}(\theta)$ is generated by $\left\{\sum_{i=0}^{l-1}\left(w^{i}-w^{\prime i}\right), \sum_{j=0}^{k-1}\left(y^{j}-y^{\prime j}\right)\right\}$. So, the generators are multiples of $w-w^{\prime}$ and $y-y^{\prime}$, respectively, and these linear differences belong to $I_{D}(\tilde{F})$. Therefore, $I_{D}(\theta) \subsetneq I_{D}(\tilde{F})$. By Proposition 4.2.2 we conclude that the canonical vector field is Lipschitz.

Example 4.2.5.

$$
F=\left(\begin{array}{ccc}
z & y+w^{2} & x^{2} \\
w^{k} & x & y
\end{array}\right)
$$

with $k \geq 2$, which is one of the normal forms obtained in Table 2. Consider the matrix of deformation

$$
\theta=\left(\begin{array}{ccc}
0 & 1 & x w+w \\
\sum_{i=0}^{k-1} w^{i} & w & w
\end{array}\right)
$$

and $\tilde{F}(u, x, y, z, w)=\left(u, F(x, y, z, w)+u \theta(x, y, z, w)\right.$. Notice that $\theta \in \frac{\operatorname{Mat}_{(2,3)}\left(\mathscr{O}_{4}\right)}{T \mathscr{G} F}, I_{D}(\theta)$ is generated by $\left\{w-w^{\prime}, w(x+1)-w^{\prime}\left(x^{\prime}+1\right), \sum_{i=0}^{k-1}\left(w^{i}-w^{\prime i}\right)\right\}$ and $I_{D}(\tilde{F})$ is generated by $\left\{z-z^{\prime},(y-\right.$ $\left.y^{\prime}\right)+w^{2}-w^{\prime 2}, x^{2}-x^{\prime 2}+u(x w+w)-u^{\prime}\left(x^{\prime} w^{\prime}+w^{\prime}\right), w^{k}-w^{\prime k}+u \sum_{i=0}^{k-1} w^{i}-u^{\prime} \sum_{i=0}^{k-1} w^{\prime i}, x+u w-x^{\prime}-$ $\left.u^{\prime} w^{\prime}, y+u w-y^{\prime}-u^{\prime} w^{\prime}\right\}$ 
Consider the curve $\phi: \mathbb{C} \longrightarrow\left(\mathbb{C} \times \mathbb{C}^{4}\right) \times\left(\mathbb{C} \times \mathbb{C}^{4}\right)$ given by $\phi(t)=(0,0,0,0,2 t, 0,0,0,0, t)$. Then, $I_{D}(\theta) \circ \phi$ is the ideal of $\mathscr{O}_{1}$ generated by $t$, and $I_{D}(\tilde{F}) \circ \phi$ is the ideal generated by $t^{2}$. Hence, $I_{D}(\theta) \circ \phi \nsubseteq I_{D}(\tilde{F}) \circ \phi$ and by the curve criterion for the integral closure of ideals we conclude that $I_{D}(\theta) \nsubseteq \overline{I_{D}(\tilde{F})}$. Therefore, Proposition 4.2.2 ensures that the canonical vector field is not Lipschitz.

Theorem 4.2.6. Consider $X$ a variety given by some $F: \mathbb{C}^{q} \longrightarrow \operatorname{Hom}\left(\mathbb{C}^{3}, \mathbb{C}^{2}\right)$ and $\tilde{F}: \mathbb{C} \times$ $\mathbb{C}^{q} \longrightarrow \mathbb{C} \times \operatorname{Hom}\left(\mathbb{C}^{3}, \mathbb{C}^{2}\right), q \in\{4,5\}$ as in 4.2 .2 , where $\theta \in \frac{\operatorname{Mat}_{(2,3)}\left(\mathscr{O}_{q}\right)}{T \mathscr{G}_{F}}$. Suppose that $X$ is a simple isolated Cohen-Macaulay variety of codimension 2.

If $F$ is of 1-jet-type $J_{q, k}$ from Table 1 then the canonical vector field is Lipschitz, otherwise it is not.

Proof. Suppose that $F$ is of 1-jet-type from Lemma 3.2 of [37]. Since $\theta \in \frac{\operatorname{Mat}_{(2,3)}\left(\mathscr{O}_{q}\right)}{T \mathscr{G} F}$ then the $q$ order 1 entries of the matrix $F$ stay unperturbed, thus the differences of the monomial generators of the maximal ideal are in $I_{D}(\tilde{F})$. In particular the ideal $I_{\Delta}$ from the diagonal satisfies the inclusion $I_{\Delta} \subseteq I_{D}(\tilde{F})$. Let $\theta_{i}, i \in\{1, \ldots, 6\}$ be the components of $\theta$. Notice that every $\left(\theta_{i}\right)_{D}$ vanishes on the diagonal $\Delta$ which implies that all the generators of $I_{D}(\theta)$ belong to $I_{\Delta}$. Therefore, $I_{D}(\theta) \subseteq I_{\Delta} \subseteq I_{D}(\tilde{F})$ and the Proposition 4.2.2 ensures that the canonical vector field is Lipschitz.

Suppose the opposite. In this case, one of the generators of the maximal ideal is not an entry of the 1 -jet of the matrix $F$. Without loss of generality, we may assume this is the first coordinate $x$. Since $\tilde{F}$ is a semiuniversal unfolding then $x-x^{\prime}$ certainly appears as a part of a generator set of $I_{D}(\theta)$. Take the curve $\phi:(\mathbb{C}, 0) \rightarrow\left(\mathbb{C} \times \mathbb{C}^{q}\right) \times\left(\mathbb{C} \times \mathbb{C}^{q}\right)$ given by $\phi(t)=(0,2 t, 0, \ldots, 0, t, 0, \ldots)$. Then $I_{D}(\tilde{F}) \circ \phi$ is generated by $t^{m}$, for some $m>1$. Since $\left(x-x^{\prime}\right) \circ$ $\phi=t$ then $\left(x-x^{\prime}\right) \circ \phi \notin I_{D}(\tilde{F}) \circ \phi$, and by the curve criterion we conclude that $x-x^{\prime} \notin \overline{I_{D}(\tilde{F})}$. Therefore, $I_{D}(\theta) \nsubseteq \overline{I_{D}(\tilde{F})}$ and the Proposition 4.2.2 ensures that the canonical vector field is not Lipschitz.

Remark 4.2.7. (Frühbis-Krüger) We can rephrase the condition on the jet-type by stating:

a) The canonical vector field is Lipschitz if the ideal of 1-minors of the matrix of X defines a reduced point.

b) The canonical vector field is not Lipschitz if the ideal of 1-minors of the matrix of X defines a fat point.

We can re-state the Theorem 4.2.6 in a more explicit way using the tables of classification of the Theorems 4.1.2 and 4.1.3.

Theorem 4.2.8. Consider $X$ a variety given by some $F: \mathbb{C}^{q} \longrightarrow \operatorname{Hom}\left(\mathbb{C}^{3}, \mathbb{C}^{2}\right)$ and $\tilde{F}: \mathbb{C} \times$ $\mathbb{C}^{q} \longrightarrow \mathbb{C} \times \operatorname{Hom}\left(\mathbb{C}^{3}, \mathbb{C}^{2}\right), q \in\{4,5\}$ as in 4.2 .2 , where $\theta \in \frac{\operatorname{Mat}_{(2,3)}\left(\mathscr{O}_{q}\right)}{T \mathscr{G} F}$. Suppose that $X$ is a simple isolated Cohen-Macaulay variety of codimension 2. 
a) Suppose $q=4$. If the normal form of $F$ is one of those first eight normal forms on 4.1.2, then the canonical vector field is Lipschitz. For the other normal forms, the canonical vector field is not Lipschitz.

b) Suppose $q=5$. If the normal form of $F$ is one of those first six normal forms on 4.1.3, then the canonical vector field is Lipschitz. For the other normal forms, the canonical vector field is not Lipschitz.

Looking to tables 2 and 3 one can realize that the canonical vector field is Lipschitz exactly when the normal form has all the generators of the maximal ideal as an entry of the matrix. 
CHAPTER

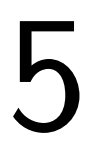

\section{CATEGORICAL ASPECTS OF THE DOUBLE STRUCTURE}

In this section, our main goal is look to the categorical properties of the double structure, under an algebraic viewpoint.

\subsection{The double homomorphism and basic properties}

Let $R$ be a ring.

Definition 5.1.1. Let $\mathscr{T}(R)$ be the category of the R-modules $M$ which are $R$-submodules of $R^{p}$, for some natural number $p$.

Let $X \subseteq \mathbb{C}^{n}$ be an analytic space and let $\mathscr{O}_{X}$ be the analytic sheaf of local rings over $X$, and let $x \in X$.

Here we work on the categories $\mathscr{T}\left(\mathscr{O}_{X, x}\right)$ and $\mathscr{T}\left(\mathscr{O}_{X \times X,(x, x)}\right)$.

Consider the projection maps $\pi_{1}, \pi_{2}: X \times X \rightarrow X$.

The first result is a quite useful tool many times when we work with the double.

Proposition 5.1.2. Let $M, N \subseteq \mathscr{O}_{X, x}^{p}$ submodules and $h, g \in \mathscr{O}_{X, x}^{p}$. Then:

a) $h=g$ if and only if $h_{D}=g_{D}$;

b) $h \in M$ if and only if $h_{D} \in M_{D}$;

c) $M \subseteq N$ if and only if $M_{D} \subseteq N_{D}$;

d) $M=N$ if and only if $M_{D}=N_{D}$. 
Proof. (a) The implication ( $\Longrightarrow$ ) is obvious. Suppose that $h_{D}=g_{D}$. In particular, $h \circ \pi_{1}=g \circ \pi_{1}$, and for all $z$ in a neighborhood of $x$ we have $h(z)=h \circ \pi_{1}(z, x)=g \circ \pi_{1}(z, x)=g(z)$, hence $h=g$.

(b) The implication $(\Longrightarrow)$ is obvious. Suppose now that $h_{D} \in M_{D}$. Then, we can write

$$
h_{D}=\sum \alpha_{i}\left(g_{i}\right)_{D}
$$

with $g_{i} \in M$ and $\alpha_{i} \in \mathscr{O}_{X \times X,(x, x)}$. In particular, $h \circ \pi_{1}=\sum \alpha_{i}\left(g_{i} \circ \pi_{1}\right)$. Taking $\alpha_{i}^{x} \in \mathscr{O}_{X, x}$ given by $\alpha_{i}^{x}(z):=\alpha_{i}(z, x), \forall i$, we get $h=\sum \alpha_{i}^{x} g_{i}$ which belongs to $M$.

(c) The implication ( $\Longrightarrow$ ) is obvious. Suppose that $M_{D} \subseteq N_{D}$. Let $h \in M$ arbitrary. Then $h_{D} \in M_{D} \subseteq N_{D}$, so by the item (b) we conclude that $h \in N$. Therefore, $M \subseteq N$.

(d) It is a straightforward consequence of the item (c).

Corollary 5.1.3. For each $\mathscr{O}_{X, x}$-submodule $M$ of $\mathscr{O}_{X, x}^{p}$, the natural map

$$
\begin{aligned}
D_{M}: M & \longrightarrow M_{D} \\
h & \longmapsto h_{D}
\end{aligned}
$$

is an injective group homomorphism. In particular, we can see $M$ as an additive subgroup of $M_{D}$.

Proof. It is a straightforward consequence of the definition of the double that $D_{M}$ is a group homomorphism. The Proposition 5.1.2 (a) gives the injectivity.

Our main goal is to give a categorical sense for the double structure. The next theorem is the key for it.

Theorem 5.1.4. Let $M \subseteq \mathscr{O}_{X, x}^{p}$ and $N \subseteq \mathscr{O}_{X, x}^{q}$ be $\mathscr{O}_{X, x}$-submodules. If $\phi: M \rightarrow N$ is an $\mathscr{O}_{X, x^{-}}$ module homomorphism then there exists a unique $\mathscr{O}_{X \times X,(x, x)}$-module homomorphism $\phi_{D}: M_{D} \rightarrow$ $N_{D}$ such that $\phi_{D}\left(h_{D}\right)=(\phi(h))_{D}, \forall h \in M$, i.e, the following diagram commutes:

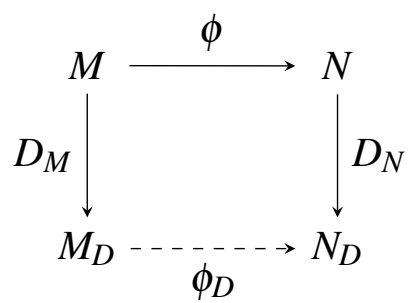

The map $\phi_{D}$ is called the double of $\phi$.

Proof. Since $M_{D}$ is generated by $\left\{h_{D} \mid h \in M\right\}$ then we can define $\phi_{D}: M_{D} \rightarrow N_{D}$ in a quite natural way: for each $u=\sum_{i} \alpha_{i}\left(h_{i}\right)_{D}$ with $\alpha_{i} \in \mathscr{O}_{X \times X,(x, x)}$ and $h_{i} \in M$ we define

$$
\phi_{D}(u):=\sum_{i} \alpha_{i}\left(\phi\left(h_{i}\right)\right)_{D}
$$


which belongs to $N_{D}$.

Claim : $\phi_{\mathbf{D}}$ is well defined. In fact, suppose that $\sum_{i} \alpha_{i}\left(h_{i}\right)_{D}=\sum_{j} \beta_{j}\left(g_{j}\right)_{D}$, with $\alpha_{i}, \beta_{j} \in$ $\mathscr{O}_{X \times X,(x, x)}$ and $h_{i}, g_{j} \in M$. So, we get two equations:

$$
\begin{aligned}
& \sum_{i} \alpha_{i}\left(h_{i} \circ \pi_{1}\right)=\sum_{j} \beta_{j}\left(g_{j} \circ \pi_{1}\right) \\
& \sum_{i} \alpha_{i}\left(h_{i} \circ \pi_{2}\right)=\sum_{j} \beta_{j}\left(g_{j} \circ \pi_{2}\right) .
\end{aligned}
$$

Take $U$ an open neighborhood of $x$ in $X$ where $\alpha_{i}, \beta_{j}$ are defined on $U \times U$, and $h_{i}, g_{j}$ are defined on $U$. For each $w \in U$ define $\alpha_{i}^{w}, \beta_{j}^{w} \in \mathscr{O}_{X, x}$ given by the germs of the maps

$$
\begin{aligned}
& \alpha_{i}^{w}: U \longrightarrow \mathbb{C} \quad \beta_{j}^{w}: U \longrightarrow \mathbb{C} \\
& z \longmapsto \alpha_{i}(z, w) \quad z \longmapsto \beta_{j}(z, w)
\end{aligned}
$$

The equation (1) implies that $\sum_{i} \alpha_{i}^{w} h_{i}=\sum_{j} \beta_{j}^{w} g_{j}, \forall w \in U$. Applying $\phi$ (which is an $\mathscr{O}_{X, x^{-}}$ homomorphism) in both sides of the last equation we get $\sum_{i} \alpha_{i}^{w} \phi\left(h_{i}\right)=\sum_{j} \beta_{j}^{w} \phi\left(g_{j}\right), \forall w \in U$. This implies that

$$
\left(\sum_{i} \alpha_{i}\left(\phi\left(h_{i}\right) \circ \pi_{1}\right)\right)(z, w)=\left(\sum_{j} \beta_{j}\left(\phi\left(g_{j}\right) \circ \pi_{1}\right)\right)(z, w)
$$

$\forall(z, w) \in U \times U$, hence

$$
\sum_{i} \alpha_{i}\left(\phi\left(h_{i}\right) \circ \pi_{1}\right)=\sum_{j} \beta_{j}\left(\phi\left(g_{j}\right) \circ \pi_{1}\right)
$$

Analogously, using the equation (2), we get

$$
\sum_{i} \alpha_{i}\left(\phi\left(h_{i}\right) \circ \pi_{2}\right)=\sum_{j} \beta_{j}\left(\phi\left(g_{j}\right) \circ \pi_{2}\right) .
$$

The equations (3) and (4) implies that

$$
\sum_{i} \alpha_{i}\left(\phi\left(h_{i}\right)\right)_{D}=\sum_{j} \beta_{j}\left(\phi\left(\left(g_{j}\right)\right)_{D}\right.
$$

and the Claim is proved.

Now, by the definition of $\phi_{D}$, it is clear that $\phi_{D}$ is an $\mathscr{O}_{X \times X,(x, x)}$-module homomorphism and is the unique satisfying the property $\phi_{D}\left(h_{D}\right)=(\phi(h))_{D}, \forall h \in M$.

From now on, all the modules are objects in $\mathscr{T}\left(\mathscr{O}_{X, x}\right)$ and their doubles are objects in $\mathscr{T}\left(\mathscr{O}_{X \times X,(x, x)}\right)$.

Notice that if $i d_{M}: M \rightarrow M$ and $i d_{M_{D}}: M_{D} \rightarrow M_{D}$ are the identity homomorphisms of $M$ and $M_{D}$, then

$$
\left(i d_{M}\right)_{D}=i d_{M_{D}}
$$

The next proposition gives us a relation between images and kernels of a module homomorphism. 
Proposition 5.1.5. Let $\phi: M \rightarrow N$ be an $\mathscr{O}_{X, x}$-module homomorphism and $\phi_{D}: M_{D} \rightarrow N_{D}$ its double. Then:

a) $\operatorname{Im}\left(\phi_{D}\right)=(\operatorname{Im}(\phi))_{D}$

b) $(\operatorname{ker}(\phi))_{D} \subseteq \operatorname{ker}\left(\phi_{D}\right)$.

Proof. (a) By definition of $\phi_{D}$, it is clear that $\operatorname{Im}\left(\phi_{D}\right) \subseteq(\operatorname{Im}(\phi))_{D}$. Now, if $g \in \operatorname{Im}(\phi)$ then we can write $g=\phi(h)$, for some $h \in M$. So, $g_{D}=(\phi(h))_{D}=\phi_{D}\left(h_{D}\right) \in \operatorname{Im}\left(\phi_{D}\right)$. Thus, $g_{D} \in \operatorname{Im}\left(\phi_{D}\right)$, $\forall g \in \operatorname{Im}(\phi)$, hence $(\operatorname{Im}(\phi))_{D} \subseteq \operatorname{Im}\left(\phi_{D}\right)$.

(b) For every $h \in \operatorname{ker}(\phi)$, we have that $\phi_{D}\left(h_{D}\right)=(\phi(h))_{D}=\left(0_{N}\right)_{D}=0_{N_{D}}$, so $h_{D} \in$ $\operatorname{ker}\left(\phi_{D}\right)$.

The next proposition shows that the double homomorphism has a good behavior with respect to sum and composition.

Proposition 5.1.6. Let $\phi, \phi^{\prime}: M \rightarrow N$ and $\gamma: N \rightarrow P$ be $\mathscr{O}_{X, x}$-module homomorphisms.

a) $\phi=\phi^{\prime}$ if and only if $\phi_{D}=\phi_{D}^{\prime}$;

b) $(\gamma \circ \phi)_{D}=\gamma_{D} \circ \phi_{D}$;

c) $\left(\phi+\phi^{\prime}\right)_{D}=\phi_{D}+\phi_{D}^{\prime}$

Proof. (a) ( $\Longrightarrow$ ) Suppose $\phi=\phi^{\prime}$. For all $h \in M, \phi_{D}\left(h_{D}\right)=(\phi(h))_{D}=\left(\phi^{\prime}(h)\right)_{D}=\phi_{D}^{\prime}\left(h_{D}\right)$. Since the module $M_{D}$ is generated by $\left\{h_{D} \mid h \in M\right\}$ then $\phi_{D}=\phi_{D}^{\prime}$.

$(\Longleftarrow)$ Suppose $\phi_{D}=\phi_{D}^{\prime}$. Given $h \in M$ arbitrary, we have $(\phi(h))_{D}=\phi_{D}\left(h_{D}\right)=\phi_{D}^{\prime}\left(h_{D}\right)=$ $\left(\phi^{\prime}(h)\right)_{D}$. By Proposition 5.1.2 (a), $\phi(h)=\phi^{\prime}(h)$. Hence, $\phi=\phi^{\prime}$.

(b) For every $h \in M$ we have $(\gamma \circ \phi)_{D}\left(h_{D}\right)=(\gamma \circ \phi(h))_{D}=\gamma_{D}\left((\phi(h))_{D}\right)=\gamma_{D} \circ \phi_{D}\left(h_{D}\right)$, which proves (b).

(c) For every $h \in M$ we have $\left(\phi+\phi^{\prime}\right)_{D}\left(h_{D}\right)=\left(\left(\phi+\phi^{\prime}\right)(h)\right)_{D}=\left(\phi(h)+\phi^{\prime}(h)\right)_{D}=$ $(\phi(h))_{D}+\left(\phi^{\prime}(h)\right)_{D}=\left(\phi_{D}+\phi_{D}^{\prime}\right)\left(h_{D}\right)$, which proves (c).

In the next corollary we compare algebraic properties of a homomorphism and its double. 
Corollary 5.1.7. Let $\phi: M \rightarrow N$ be an $\mathscr{O}_{X, x}$-module homomorphism. Then:

a) $\phi: M \rightarrow N$ is surjective if and only if $\phi_{D}: M_{D} \rightarrow N_{D}$ is surjective;

b) If $\phi_{D}: M_{D} \rightarrow N_{D}$ is injective then $\phi: M \rightarrow N$ is injective;

c) $\phi: M \rightarrow N$ is an $\mathscr{O}_{X, x}$-isomorphism if and only if $\phi_{D}: M_{D} \rightarrow N_{D}$ is an $\mathscr{O}_{X \times X,(x, x)^{-}}$ isomorphism;

d) $\phi: M \rightarrow N$ is the zero homomorphism if and only if $\phi_{D}: M_{D} \rightarrow N_{D}$ is the zero homomorphism.

Proof. (a) By Propositions 5.1.2 (d) and 5.1.5 (a) we have: $\phi$ is surjective $\Longleftrightarrow \operatorname{Im}(\phi)=N$ $\Longleftrightarrow(\operatorname{Im}(\phi))_{D}=N_{D} \Longleftrightarrow \operatorname{Im}\left(\phi_{D}\right)=N_{D} \Longleftrightarrow \phi_{D}$ is surjective.

(b) If $\phi_{D}$ is injective then $\operatorname{ker}\left(\phi_{D}\right)=0_{M_{D}}$, and the Proposition 5.1.5 (b) implies that $(\operatorname{ker}(\phi))_{D} \subseteq \operatorname{ker}\left(\phi_{D}\right)=0_{M_{D}}=\left(0_{M}\right)_{D}$. By Proposition 5.1.2 (c) we conclude that $\operatorname{ker}(\phi)=0_{M}$, hence $\phi$ is injective.

(c) $(\Longrightarrow)$ Since $\phi: M \rightarrow N$ is an isomorphism then there exists an $\mathscr{O}_{X, x}$-homomorphism $\gamma: N \rightarrow M$ such that $\gamma \circ \phi=i d_{M}$ and $\phi \circ \gamma=i d_{N}$, then, by Proposition 5.1.6 (b) we have $(\gamma)_{D} \circ(\phi)_{D}=i d_{M_{D}}$ and $(\phi)_{D} \circ(\gamma)_{D}=i d_{N_{D}}$. Hence, $\phi_{D}$ is an isomorphism.

$(\Longleftarrow)$ It follows immediately from (a) and (b).

(d) By Propositions 5.1.2 (d) and 5.1.5 (a) we have: $\phi$ is the zero homomorphism $\Longleftrightarrow$ $\operatorname{Im}(\phi)=0_{N} \Longleftrightarrow(\operatorname{Im}(\phi))_{D}=\left(0_{N}\right)_{D} \Longleftrightarrow \operatorname{Im}\left(\phi_{D}\right)=0_{N_{D}} \Longleftrightarrow \phi_{D}$ is the zero homomorphism.

Definition 5.1.8. We say that an $\mathscr{O}_{X, x}$-homomorphism $\phi: M \subseteq \mathscr{O}_{X, x}^{p} \rightarrow N \subseteq \mathscr{O}_{X, x}^{q}$ is induced by a $q \times p$ matrix if there exists $A \in \operatorname{Mat}_{q \times p}\left(\mathscr{O}_{X, x}\right)$ such that $\phi(h)=A \cdot h, \forall h \in M$.

Lemma 5.1.9. An $\mathscr{O}_{X, x}$-homomorphism $\phi: M \subseteq \mathscr{O}_{X, x}^{p} \rightarrow N \subseteq \mathscr{O}_{X, x}^{q}$ is induced by a $q \times p$ matrix if and only if there exists an $\mathscr{O}_{X, x}$-homomorphism $\tilde{\phi}: \mathscr{O}_{X, x}^{p} \rightarrow \mathscr{O}_{X, x}^{q}$ such that $\tilde{\phi}(M) \subseteq N$ and $\left.\tilde{\phi}\right|_{M}=\phi$.

Proof. $(\Longrightarrow)$ By hypothesis there exists a $q \times p$ matrix $A$ with entries in $\mathscr{O}_{X, x}$ such that $\phi(h)=$ $A \cdot h, \forall h \in M$. From this matrix $A$, we can define $\tilde{\phi}: \mathscr{O}_{X, x}^{p} \rightarrow \mathscr{O}_{X, x}^{q}$ given by $\tilde{\phi}(g):=A \cdot g$, which is an $\mathscr{O}_{X, x}$-homomorphism. Clearly, $\left.\tilde{\phi}\right|_{M}=\phi$, and for all $h \in M$ we have $\tilde{\phi}(h)=\phi(h) \in N$, so $\tilde{\phi}(M) \subseteq N$.

$(\Longleftarrow)$ Let $e_{1}, \ldots, e_{p}$ be the canonical elements in $\mathscr{O}_{X, x}^{p}$. Let $A$ be the $q \times p$ matrix whose columns are $\phi\left(e_{1}\right), \ldots, \phi\left(e_{p}\right)$. Then $\tilde{\phi}(g)=A \cdot g, \forall g \in \mathscr{O}_{X, x}^{p}$. Since $\left.\tilde{\phi}\right|_{M}=\phi$ then $\phi(h)=\tilde{\phi}(h)=$ $A \cdot h, \forall h \in M$. Therefore, $\phi$ is induced by a $q \times p$ matrix. 
Proposition 5.1.10. If $\phi: M \subseteq \mathscr{O}_{X, x}^{p} \rightarrow N \subseteq \mathscr{O}_{X, x}^{q}$ is an $\mathscr{O}_{X, x}$-homomorphism induced by a $q \times p$ matrix then

$$
\phi_{D}: M_{D} \subseteq \mathscr{O}_{X \times X,(x, x)}^{2 p} \rightarrow N_{D} \subseteq \mathscr{O}_{X \times X,(x, x)}^{2 q}
$$

is an $\mathscr{O}_{X \times X,(x, x)}$-homomorphism induced by a $2 q \times 2 p$ matrix.

Proof. By hypothesis there exists a $q \times p$ matrix $A$ with entries in $\mathscr{O}_{X, x}$ such that $\phi(h)=A \cdot h, \forall h \in$ $M$. Then, for all $h \in M$ we have $\phi_{D}\left(h_{D}\right)=\left[\begin{array}{l}\phi(h) \circ \pi_{1} \\ \phi(h) \circ \pi_{2}\end{array}\right]=\left[\begin{array}{l}(A \cdot h) \circ \pi_{1} \\ (A \cdot h) \circ \pi_{2}\end{array}\right]=\left[\begin{array}{l}\left(A \circ \pi_{1}\right) \cdot\left(h \circ \pi_{1}\right) \\ \left(A \circ \pi_{2}\right) \cdot\left(h \circ \pi_{2}\right)\end{array}\right]$.

So, taking the $2 q \times 2 p$ matrix

$$
B:=\left[\begin{array}{cc}
A \circ \pi_{1} & 0_{q \times p} \\
0_{q \times p} & A \circ \pi_{2}
\end{array}\right]
$$

we conclude that $\phi_{D}\left(h_{D}\right)=B \cdot h_{D}$, and the proposition is proved, once $M_{D}$ is generated by $h_{D}$, $h \in M$.

As an application of the double homomorphism, we prove in the next theorem that the double structure is compatible with finite direct sum of modules.

Theorem 5.1.11. Let $M \subseteq \mathscr{O}_{X, x}^{p}$ and $N \subseteq \mathscr{O}_{X, x}^{q}$ be $\mathscr{O}_{X, x}$-submodules. Then

$$
(M \oplus N)_{D} \cong M_{D} \oplus N_{D}
$$

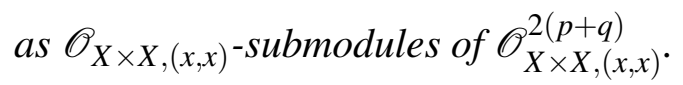

Furthermore, there exists an isomorphism

$$
\eta:(M \oplus N)_{D} \longrightarrow M_{D} \oplus N_{D}
$$

such that $\eta\left((h, g)_{D}\right)=\left(h_{D}, g_{D}\right)$, for all $h \in M$ and $g \in N$.

Proof. Consider the canonical projections and inclusions:

$$
\begin{aligned}
& \psi_{1}: M \oplus N \quad \longrightarrow \quad M \quad \psi_{2}: M \oplus N \quad \longrightarrow \quad N \\
& (h, g) \longmapsto h \quad h \quad(h, g) \longmapsto g
\end{aligned}
$$

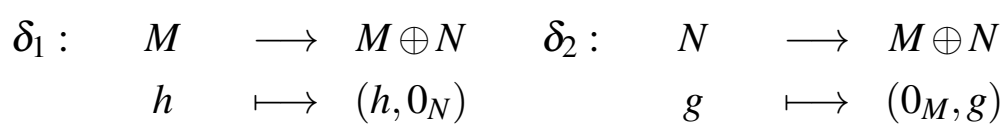

Thus, we get the double homomorphism of each one above:

$$
\begin{aligned}
& \left(\psi_{1}\right)_{D}:(M \oplus N)_{D} \quad \longrightarrow \quad M_{D} \quad\left(\psi_{2}\right)_{D}:(M \oplus N)_{D} \quad \longrightarrow N_{D} \\
& \left(\delta_{1}\right)_{D}: M_{D} \longrightarrow(M \oplus N)_{D} \quad\left(\delta_{2}\right)_{D}: N_{D} \longrightarrow(M \oplus N)_{D}
\end{aligned}
$$


Define:

$$
\begin{array}{rlc}
\eta:(M \oplus N)_{D} & \longrightarrow & M_{D} \oplus N_{D} \\
w & \longmapsto & \left(\left(\psi_{1}\right)_{D}(w),\left(\psi_{2}\right)_{D}(w)\right) \\
\delta: M_{D} \oplus N_{D} & \longrightarrow & (M \oplus N)_{D} \\
(u, v) & \longmapsto & \left(\delta_{1}\right)_{D}(u)+\left(\delta_{2}\right)_{D}(v)
\end{array}
$$

which are $\mathscr{O}_{X \times X,(x, x)}$-module homomorphisms.

Claim 1: $\eta\left((h, g)_{D}\right)=\left(h_{D}, g_{D}\right)$, for all $h \in M$ and $g \in N$.

In fact, $\eta\left((h, g)_{D}\right)=\left(\left(\psi_{1}\right)_{D}\left((h, g)_{D}\right),\left(\psi_{2}\right)_{D}\left((h, g)_{D}\right)\right)=\left(\left(\psi_{1}(h, g)\right)_{D},\left(\psi_{2}(h, g)\right)_{D}\right)=$ $\left(h_{D}, g_{D}\right)$.

Claim 2: $\delta\left(h_{D}, g_{D}\right)=(h, g)_{D}$, for all $h \in M$ and $g \in N$.

In fact, $\delta\left(h_{D}, g_{D}\right)=\left(\delta_{1}\right)_{D}\left(h_{D}\right)+\left(\delta_{2}\right)_{D}\left(g_{D}\right)=\left(\delta_{1}(h)\right)_{D}+\left(\delta_{2}(g)\right)_{D}=\left(h, 0_{N}\right)_{D}+\left(0_{M}, g\right)_{D}=$ $\left(\left(h, 0_{N}\right)+\left(0_{M}, g\right)\right)_{D}=(h, g)_{D}$.

By the Claims 1 and 2 we have that $\delta \circ \eta\left((h, g)_{D}\right)=(h, g)_{D}, \eta \circ \delta\left(h_{D}, 0_{N_{D}}\right)=\left(h_{D}, 0_{N_{D}}\right)$ and $\eta \circ \delta\left(0_{M_{D}}, g_{D}\right)=\left(0_{M_{D}}, g_{D}\right)$, for all $h \in M$ and $g \in N$.

Since $\left\{(h, g)_{D} \mid h \in M\right.$ and $\left.g \in N\right\}$ is a generator set of $(M \oplus N)_{D}$ and $\left\{\left(h_{D}, 0_{N_{D}}\right),\left(0_{M_{D}}, g_{D}\right) \mid h \in M\right.$ and $\left.g \in N\right\}$ is a generator set of $M_{D} \oplus N_{D}$ then we conclude that $\delta \circ \eta=i d_{(M \oplus N)_{D}}$ and $\eta \circ \delta=i d_{M_{D} \oplus N_{D}}$, which finishes the proof of the theorem.

Corollary 5.1.12. Let $M_{i} \subseteq \mathscr{O}_{X, x}^{p_{i}}$ be $\mathscr{O}_{X, x}$-submodules, for each $i \in\{1, \ldots, r\}$. Then

$$
\left(M_{1} \oplus \ldots \oplus M_{r}\right)_{D} \cong\left(M_{1}\right)_{D} \oplus \ldots \oplus\left(M_{r}\right)_{D}
$$

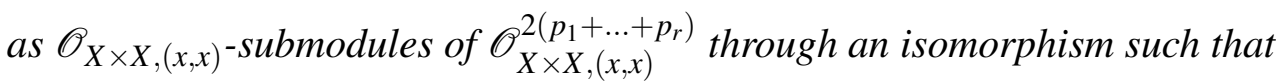

$$
\left(h_{1}, \ldots, h_{r}\right)_{D} \longmapsto\left(\left(h_{1}\right)_{D}, \ldots,\left(h_{r}\right)_{D}\right)
$$

for all $h_{i} \in M_{i}$.

Proof. Induction on $r$ and use the previous theorem.

The next proposition compares the length of a module and its double.

Proposition 5.1.13. Let $M \subseteq N$ be $\mathscr{O}_{X, x}$-submodules of $\mathscr{O}_{X, x}^{p}$.

a) If $M_{D}$ has finite length then $M$ has finite length and $\ell(M) \leq \ell\left(M_{D}\right)$;

b) If $M_{D}$ has finite colength in $N_{D}$ then $M$ has finite colength in $N$. 
Proof. (a) If $r \in \mathbb{N}$ and $\left(M_{i}\right)_{i=0}^{r}$ is an ascending series of $M$, then $\left(\left(M_{i}\right)_{D}\right)_{i=0}^{r}$ is an ascending series of $M_{D}$, which has finite length. Thus, $r \leq \ell\left(M_{D}\right)$. Therefore $\ell(M)$ is finite and $\ell(M) \leq \ell\left(M_{D}\right)$.

(b) Let $r \in \mathbb{N}$ and consider an arbitrary ascending series of $\frac{N}{M}$ of length $r$. This series can be given on the form

$$
\frac{N_{0}}{M} \subsetneq \frac{N_{1}}{M} \subsetneq \ldots \subsetneq \frac{N_{r-1}}{M} \subsetneq \frac{N_{r}}{M}=\frac{N}{M}
$$

where $N_{0} \subsetneq N_{1} \subsetneq \ldots \subsetneq N_{r-1} \subsetneq N_{r}=N$ are $\mathscr{O}_{X, x}$-submodules of $N$ which contain $M$. Then,

$$
\frac{\left(N_{0}\right)_{D}}{\left(M_{D}\right)} \subsetneq \frac{\left(N_{1}\right)_{D}}{M_{D}} \subsetneq \ldots \subsetneq \frac{\left(N_{r-1}\right)_{D}}{M_{D}} \subsetneq \frac{\left(N_{r}\right)_{D}}{M_{D}}=\frac{N_{D}}{M_{D}}
$$

is an ascending series of $\frac{N_{D}}{M_{D}}$, which has finite length by hypothesis. Hence, $r \leq \ell\left(\frac{N_{D}}{M_{D}}\right)$ and $\ell\left(\frac{N}{M}\right)$ is finite.

We want to use the double homomorphism in order to get an equivalence between the second and third Lipschitz saturations.

The next proposition gives the "persistence" of the integral closure of modules.

Proposition 5.1.14. Let $\varphi: M \subseteq \mathscr{O}_{X, x}^{p} \rightarrow N \subseteq \mathscr{O}_{X, x}^{q}$ be a homomorphism of $\mathscr{O}_{X, x}$-modules which can be extended to a homomorphism $\tilde{\varphi}: \mathscr{O}_{X, x}^{p} \rightarrow c O_{X, x}^{q}$, given by $\tilde{\varphi}(h)=A \cdot h$, where $A$ is a $q \times p$ matrix with entries in $\mathscr{O}_{X, x}$. Let $h \in \mathscr{O}_{X, x}^{p}$.

a) If $h \in \bar{M}$ then $\tilde{\varphi}(h) \in \overline{\varphi(M)}$;

b) Suppose $q=p$. If $A$ is an invertible matrix and $\varphi$ is an isomorphism of $\mathscr{O}_{X, x}$-modules then:

$$
h \in \bar{M} \text { if and only if } \tilde{\varphi}(h) \in \overline{\varphi(M)}
$$

c) Suppose $q=p$ and $\varphi$ is injective. If $\tilde{\varphi}(h) \in \overline{\varphi(M)}$ then $h \in \bar{M}$.

Proof. (a) Let $\phi:(\mathbb{C}, 0) \rightarrow(X, x)$ be an arbitrary analytic curve. By hypothesis $\phi^{*}(h) \in \phi^{*}(M)$ and we can write $\phi^{*}(h)=\sum \alpha_{i} \phi^{*}\left(g_{i}\right)$, for some $g_{i} \in M$ and $\alpha_{i} \in \mathscr{O}_{\mathbb{C}, 0}$. Thus: $\tilde{\varphi}(h) \circ \phi=(A \cdot h) \circ$ $\phi=[A \circ \phi] \cdot[h \circ \phi]=[A \circ \phi] \cdot \sum \alpha_{i} \phi^{*}\left(g_{i}\right)=\sum \alpha_{i}\left(\left[A \cdot g_{i}\right] \circ \phi\right) \in \phi^{*}(\varphi(M))$.

Hence, $\tilde{\varphi}(h) \in \overline{\varphi(M)}$.

(b) It suffices apply (a) in $\tilde{\varphi}^{-1}$.

(c) It suffices consider the isomorphism $\hat{\varphi}: M \rightarrow \varphi(M)$ given by $\hat{\varphi}(h)=\varphi(h)$ and apply the item (b).

For each $i \in\{1, \ldots, p\}$ consider the $i$-th canonical global section of the vector bundle $\operatorname{Hom}\left(\mathbb{C}^{p}, \mathbb{C}\right)$

$$
\xi_{i}: X \rightarrow \operatorname{Hom}\left(\mathbb{C}^{p}, \mathbb{C}\right)
$$

given by $\xi_{i}(x)=(0, . ., 1, \ldots 0)$, where 1 is on the $i$-th place.

Notice that if $M$ is an $\mathscr{O}_{X, x}$-submodule of $\mathscr{O}_{X, x}^{p}$ then $M \subseteq \xi_{1} \cdot M \oplus \ldots \oplus \xi_{p} \cdot M$. 
Theorem 5.1.15. Let $M \subseteq \mathscr{O}_{X, x}^{p}$ be a submodule. Suppose that $M_{D}$ is a reduction of $\left(\xi_{1} \cdot M \oplus \ldots \oplus \xi_{p} \cdot M\right)_{D}$. Then, $M_{S_{2}}=M_{S_{3}}$.

Proof. Consider the inclusion $i: M \rightarrow \xi_{1} \cdot M \oplus \ldots \oplus \xi_{p} \cdot M$. Then we can consider the inclusion $i_{D}: M_{D} \rightarrow\left(\xi_{1} \cdot M \oplus \ldots \oplus \xi_{p} \cdot M\right)_{D}$ which is induced by an invertible $2 p \times 2 p$ matrix. By Corollary 5.1.12 there is an isomorphism

$$
\gamma:\left(\xi_{1} \cdot M \oplus \ldots \oplus \xi_{p} \cdot M\right)_{D} \rightarrow\left(\xi_{1} \cdot M\right)_{D} \oplus \ldots \oplus\left(\xi_{p} \cdot M\right)_{D}
$$

and by the proof of this corollary, this isomorphism is induced by an invertible $2 p \times 2 p$ matrix. Taking the composition of $i_{D}$ with $\gamma$, we get an injective homomorphism

$$
\eta: M_{D} \rightarrow\left(\xi_{1} \cdot M\right)_{D} \oplus \ldots \oplus\left(\xi_{p} \cdot M\right)_{D}
$$

induced by an invertible $2 p \times 2 p$ matrix $B$ which extends to the isomorphism

$$
\tilde{\eta}: \mathscr{O}_{X \times X,(x, x)}^{2 p} \rightarrow \mathscr{O}_{X \times X,(x, x)}^{2 p}
$$

given by the multiplication by $B$, which satisfies the property

$$
\left(g_{1}, \ldots, g_{p}\right)_{D} \mapsto\left(\left(g_{1}\right)_{D}, \ldots,\left(g_{p}\right)_{D}\right)
$$

By Proposition 3.1.22 we have the inclusion $M_{S_{2}} \subseteq M_{S_{3}}$. So, it suffices to check the another inclusion.

Let $h \in M_{S_{3}}$. In particular, $\left(\xi_{i} \cdot h\right)_{D} \in \overline{\left(\xi_{i} \cdot M\right)_{D}}, \forall i \in\{1, \ldots, p\}$. Let $\phi:(\mathbb{C}, 0) \rightarrow(X \times$ $X,(x, x))$ be an arbitrary analytic curve. Then $\phi^{*}\left(\left(\xi_{i} \cdot h\right)_{D}\right) \in \phi^{*}\left(\left(\xi_{i} \cdot M\right)_{D}\right), \forall i \in\{1, \ldots, p\}$ and $\phi^{*}\left(\tilde{\eta}\left(h_{D}\right)\right)=\phi^{*}\left(\left(\xi_{1} \cdot h\right)_{D}, \ldots,\left(\xi_{p} \cdot h\right)_{D}\right)=\left(\phi^{*}\left(\left(\xi_{1} \cdot h\right)_{D}\right), \ldots, \phi^{*}\left(\left(\xi_{p} \cdot h\right)_{D}\right)\right)$ which belongs to $\phi^{*}\left(\left(\xi_{1} \cdot M\right)_{D} \oplus \ldots \oplus\left(\xi_{p} \cdot M\right)_{D}\right)$. Hence, $\tilde{\eta}\left(h_{D}\right) \in \overline{\left(\xi_{1} \cdot M\right)_{D} \oplus \ldots \oplus\left(\xi_{p} \cdot M\right)_{D}}$.

Since $M_{D}$ is a reduction of $\left(\xi_{1} \cdot M \oplus \ldots \oplus \xi_{p} \cdot M\right)_{D}$ then by the previous proposition we have that $\eta\left(M_{D}\right)$ is a reduction of $\left(\xi_{1} \cdot M\right)_{D} \oplus \ldots \oplus\left(\xi_{p} \cdot M\right)_{D}$. Thus, $\tilde{\eta}\left(h_{D}\right) \in \overline{\eta\left(M_{D}\right)}$, and by the previous proposition we conclude that $h_{D} \in \overline{M_{D}}$, therefore $h \in M_{S_{2}}$.

Example 5.1.16. Notice that any p-symmetric submodule $M \subseteq \mathscr{O}_{X, x}^{p}$ satisfies the hypothesis of the previous theorem, so $M_{S_{2}}=M_{S_{3}}$. Here, p-symmetric means that given $\left(h_{1}, \ldots, h_{p}\right) \in M$ arbitrary, then $\left(h_{\sigma(1)}, \ldots, h_{\sigma(p)}\right) \in M$ for every p-permutation $\sigma$. Observe that is equivalent to $M=\xi_{1} \cdot M \oplus \ldots \oplus \xi_{p} \cdot M$. 


\subsection{Homological aspects of the double structure}

In this section we define the double chain of a given chain complex, and we compare homological properties between these complexes.

First, we need the following result.

Proposition 5.2.1. Let

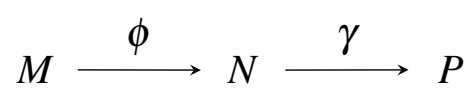

be a sequence of $\mathscr{O}_{X, x}$-module homomorphism and consider the double sequence

$$
\begin{gathered}
M_{D} \stackrel{\phi_{D}}{\longrightarrow} N_{D} \stackrel{\gamma_{D}}{\longrightarrow} P_{D} \\
\text { If } \operatorname{Im}(\phi) \subseteq \operatorname{ker}(\gamma) \text { then } \operatorname{Im}\left(\phi_{D}\right) \subseteq \operatorname{ker}\left(\gamma_{D}\right) .
\end{gathered}
$$

Proof. Since $\operatorname{Im}(\phi) \subseteq \operatorname{ker}(\gamma)$ then $(\operatorname{Im}(\phi))_{D} \subseteq(\operatorname{ker}(\gamma))_{D}$. Hence, $\operatorname{Im}\left(\phi_{D}\right)=(\operatorname{Im}(\phi))_{D} \subseteq(\operatorname{ker}(\gamma))_{D} \subseteq$ $\operatorname{ker}\left(\gamma_{D}\right)$.

We realize that the double homomorphism gives a natural way to study the homology of the double structure.

Definition 5.2.2 (The double chain complex). Let $\mathscr{C}=\left(M_{\bullet}, \phi_{\bullet}\right)$ be a chain complex in $\mathscr{T}\left(\mathscr{O}_{X, x}\right)$. We define

$$
\mathscr{C}_{D}:=\left(\left(M_{\bullet}\right)_{D},\left(\phi_{\bullet}\right)_{D}\right)
$$

and by Proposition 5.2.1 we have that $\mathscr{C}_{D}$ is a chain complex in $\mathscr{T}\left(\mathscr{O}_{X \times X,(x, x)}\right)$. The chain complex $\mathscr{C}_{D}$ is called the double of $\mathscr{C}$.

Proposition 5.2.3. Let $\mathscr{C}=\left(M_{\bullet}, \phi_{\bullet}\right)$ be a chain complex. If $\mathscr{C}_{D}$ is an exact sequence then $\mathscr{C}$ is an exact sequence. In other words, if $\mathscr{C}_{D}$ has trivial homology then $\mathscr{C}$ has trivial homology.

Proof. Let $i \in \mathbb{Z}$ be arbitrary. We have the commutative diagram:

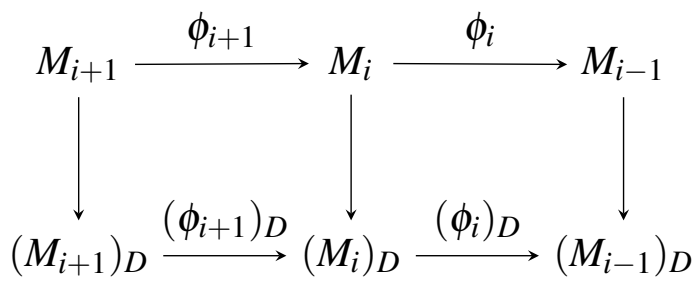


where the vertical arrows are the canonical morphisms. We already know that $\operatorname{Im}\left(\phi_{i+1}\right) \subseteq \operatorname{ker}\left(\phi_{i}\right)$. Since $\mathscr{C}_{D}$ is an exact sequence then $\operatorname{Im}\left(\left(\phi_{i+1}\right)_{D}\right)=\operatorname{ker}\left(\left(\phi_{i}\right)_{D}\right)$. By Proposition 5.1.5 we have $\left(\operatorname{ker}\left(\phi_{i}\right)\right)_{D} \subseteq \operatorname{ker}\left(\left(\phi_{i}\right)_{D}\right)=\operatorname{Im}\left(\left(\phi_{i+1}\right)_{D}\right)=\left(\operatorname{Im}\left(\phi_{i+1}\right)\right)_{D}$. By Proposition 5.1.2 (c), we conclude that $\operatorname{ker}\left(\phi_{i}\right) \subseteq \operatorname{Im}\left(\phi_{i+1}\right)$. Therefore, $\operatorname{Im}\left(\phi_{i+1}\right)=\operatorname{ker}\left(\phi_{i}\right)$.

Proposition 5.2.4. Let $\mathscr{C}=\left(M_{\bullet}, \phi_{\bullet}\right)$ and $\mathscr{C}^{\prime}=\left(M_{\bullet}^{\prime}, \phi_{\bullet}^{\prime}\right)$ be chain complexes. If $\alpha: \mathscr{C} \longrightarrow \mathscr{C}^{\prime}$ is a chain complex morphism then $\alpha_{D}: \mathscr{C}_{D} \longrightarrow \mathscr{C}_{D}^{\prime}$ given by $\left\{\left(\alpha_{i}\right)_{D}:\left(M_{i}\right)_{D} \rightarrow\left(M_{i}^{\prime}\right)_{D} \mid i \in \mathbb{Z}\right\}$ is a chain complex morphism, called the the double morphism of $\alpha$.

Proof. Let $i \in \mathbb{Z}$. We have the following commutative diagram

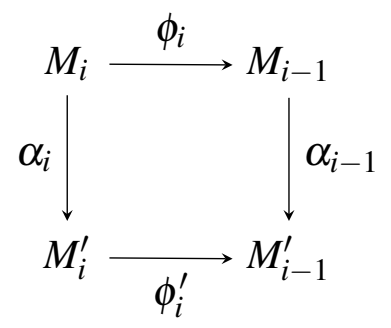

By Proposition 5.1.6 (b) follows that $\left(\phi_{i}^{\prime}\right)_{D} \circ\left(\alpha_{i}\right)_{D}=\left(\phi_{i}^{\prime} \circ \alpha_{i}\right)_{D}=\left(\alpha_{i-1} \circ \phi_{i}\right)_{D}=\left(\alpha_{i-1}\right)_{D} \circ$ $\left(\phi_{i}\right)_{D}$, and the following diagram is also commutative:

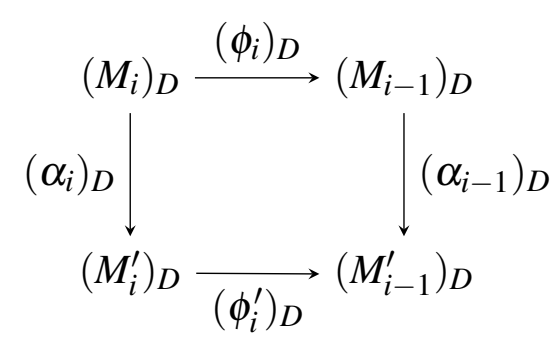

Corollary 5.2.5. If $\alpha: \mathscr{C} \longrightarrow \mathscr{C}^{\prime}$ and $\beta: \mathscr{C}^{\prime} \longrightarrow \mathscr{C}^{\prime \prime}$ are chain morphisms then

$$
(\beta \circ \alpha)_{D}=\beta_{D} \circ \alpha_{D}
$$

Proof. It is a straightforward consequence of the Proposition 5.1.6 (b).

Now, we get some results related to chain homotopy.

Let $\mathscr{C}=\left(M_{\bullet}, \phi_{\bullet}\right)$ and $\mathscr{C}^{\prime}=\left(M_{\bullet}^{\prime}, \phi_{\bullet}^{\prime}\right)$ be chain complexes. Let $\mu: \mathscr{C} \rightarrow \mathscr{C}^{\prime}$ be a homomorphism of degree 1 , i.e, $\mu$ is a collection of $\mathscr{O}_{X, x}$-module homomorphisms $\left\{\mu_{i}: M_{i} \rightarrow\right.$ $\left.M_{i+1}^{\prime} \mid i \in \mathbb{Z}\right\}$. We know this homomorphism induces a chain morphism $\tilde{\mu}: \mathscr{C} \rightarrow \mathscr{C}^{\prime}$ given by $\left\{\tilde{\mu}_{i}: M_{i} \rightarrow M_{i}^{\prime} \mid i \in \mathbb{Z}\right\}$, where $\tilde{\mu}_{i}:=\phi_{i+1}^{\prime} \circ \mu_{i}+\mu_{i-1} \circ \phi_{i}, \forall i \in \mathbb{Z}$.

If $\alpha, \beta: \mathscr{C} \rightarrow \mathscr{C}^{\prime}$ are chain morphisms, remember that $\mu: \mathscr{C} \rightarrow \mathscr{C}^{\prime}$ is defined as a homotopy between $\alpha$ and $\beta$ when $\tilde{\mu}=\alpha-\beta$, and we denote $\alpha \widetilde{\mu} \beta$. 
Lemma 5.2.6. Consider $\mu_{D}: \mathscr{C}_{D} \rightarrow \mathscr{C}_{D}^{\prime}$ the homomorphism of degree 1 given by the double homomorphisms of $\mu: \mathscr{C} \rightarrow \mathscr{C}^{\prime}$. Then $\widetilde{\mu_{D}}=(\tilde{\mu})_{D}$.

Proof. For all $i \in \mathbb{Z}$ we have $\left(\widetilde{\mu_{D}}\right)_{i}=\left(\phi_{i+1}^{\prime}\right)_{D} \circ\left(\mu_{i}\right)_{D}+\left(\mu_{i-1}\right)_{D} \circ\left(\phi_{i}\right)_{D}$ $=\left(\phi_{i+1}^{\prime} \circ \mu_{i}+\mu_{i-1} \circ \phi_{i}\right)_{D}=\left(\tilde{\mu}_{i}\right)_{D}$, and the lemma is proved.

Proposition 5.2.7. Let $\alpha, \beta: \mathscr{C} \rightarrow \mathscr{C}^{\prime}$ be chain morphisms and $\mu: \mathscr{C} \rightarrow \mathscr{C}^{\prime}$ a homomorphism of degree 1. Then: $\mu$ is a homotopy between $\alpha$ and $\beta$ if and only if $\mu_{D}$ is a homotopy between $\alpha_{D}$ and $\beta_{D}$.

Proof. We have that $\mu$ is a homotopy between $\alpha$ and $\beta$ if and only if $\tilde{\mu}=\alpha-\beta$. By Proposition 5.1.6 (a) and (c) and the previous lemma we have: $\tilde{\mu}=\alpha-\beta \Longleftrightarrow(\tilde{\mu})_{D}=(\alpha-\beta)_{D} \Longleftrightarrow$ $\widetilde{\mu_{D}}=\alpha_{D}-\beta_{D} \Longleftrightarrow \mu_{D}$ is a homotopy between $\alpha_{D}$ and $\beta_{D}$.

Corollary 5.2.8. If $\alpha: \mathscr{C} \rightarrow \mathscr{C}^{\prime}$ is a chain homotopy equivalence then $\alpha_{D}: \mathscr{C}_{D} \rightarrow \mathscr{C}_{D}^{\prime}$ is a chain homotopy equivalence.

Proof. By hypothesis there exists a chain morphism $\beta: \mathscr{C}^{\prime} \rightarrow \mathscr{C}$ such that $\beta \circ \alpha \simeq i d_{\mathscr{C}}$ and $\alpha \circ \beta \simeq i d_{\mathscr{C}^{\prime}}$. By the previous proposition, we have $(\beta \circ \alpha)_{D} \simeq\left(i d_{\mathscr{C}}\right)_{D}$ and $(\alpha \circ \beta)_{D} \simeq\left(i d_{\mathscr{C}^{\prime}}\right)_{D}$, and therefore $(\beta)_{D} \circ(\alpha)_{D} \simeq i d_{\mathscr{C}_{D}}$ and $\alpha_{D} \circ \beta_{D} \simeq i d_{\mathscr{C}_{D}^{\prime}}$.

Corollary 5.2.9. If $\mathscr{C}$ is a contractible chain complex then $\mathscr{C}_{D}$ is also contractible.

Proof. Since $\mathscr{C}$ is contractible then $i d_{\mathscr{C}} \simeq 0_{\mathscr{C}}$, and the Proposition 5.2.7 implies that $\left(i d_{\mathscr{C}}\right)_{D} \simeq$ $\left(0_{\mathscr{C}}\right)_{D}$, thus $i d_{\mathscr{C}_{D}} \simeq 0_{\mathscr{C}_{D}}$. Hence, $\mathscr{C}_{D}$ is contractible.

Notice the nice relation between the Proposition 5.2.3 and Corollary 5.2.9. We already know every contractible chain complex is an exact sequence. The Proposition 5.2.3 says that the exactness on the double level implies the exactness on the "single" level. The Corollary 5.2.9, which treats about contractible (stronger than exactness), says the opposite.

It is clear that all the results obtained in this section can be naturally translated to the cohomology language. 


\subsection{The Double category}

Let us define the category $\mathscr{D}\left(\mathscr{O}_{X, x}\right)$.

The objects of $\mathscr{D}\left(\mathscr{O}_{X, x}\right)$ consist of the double of modules in $\mathscr{T}\left(\mathscr{O}_{X, x}\right)$. Given $M_{D}, N_{D}$ objects in $\mathscr{D}\left(\mathscr{O}_{X, x}\right)$, we define

$$
\operatorname{Mor}\left(M_{D}, N_{D}\right):=\left\{\phi_{D}: M_{D} \rightarrow N_{D} \mid \phi: M \rightarrow N \text { is an } \mathscr{O}_{X, x} \text {-module homomorphism }\right\}
$$

Working with the standard composition of maps, we have the category $\mathscr{D}\left(\mathscr{O}_{X, x}\right)$, called the Double category of $(\mathbf{X}, \mathbf{x})$.

The morphisms in $\mathscr{D}\left(\mathscr{O}_{X, x}\right)$ are called $\mathscr{O}_{X, x}$-double morphisms. Observe that the $\mathscr{O}_{X, x^{-}}$ doubles morphisms are $\mathscr{O}_{X \times X,(x, x)}$-homomorphisms with an addictional property: they preserve the double structure.

Notice that $\mathscr{D}\left(\mathscr{O}_{X, x}\right)$ is a subcategory of $\mathscr{T}\left(\mathscr{O}_{X \times X,(x, x)}\right)$.

Theorem 5.3.1. The covariant functor

$$
\begin{aligned}
& \text { D : } \quad \mathscr{T}\left(\mathscr{O}_{X, x}\right) \quad \longrightarrow \quad \mathscr{D}\left(\mathscr{O}_{X, x}\right) \\
& M \quad \longmapsto \quad M_{D} \\
& \phi: M \rightarrow N \longmapsto \phi_{D}: M_{D} \rightarrow N_{D}
\end{aligned}
$$

is an isomorphism of categories.

Proof. The Proposition 5.1.2 (d) proves that the map between the objects is a bijection, and the Proposition 5.1.6 (a) proves that the map between the morphisms is a bijection. Hence, $\mathbf{D}$ is an isomorphism of categories.

Corollary 5.3.2. $\mathscr{T}\left(\mathscr{O}_{X, x}\right)$ can be seen as a subcategory of $\mathscr{T}\left(\mathscr{O}_{X \times X,(x, x)}\right)$.

The Theorem 5.3.1 implies that $\mathscr{T}\left(\mathscr{O}_{X, x}\right)$ and $\mathscr{D}\left(\mathscr{O}_{X, x}\right)$ are essentially the same category, so they have the same behavior in all of the categorical statements. But, one of them is reasonable to emphasize, in the next corollary, which is interesting to compare with the result obtained in the Corollary 5.1.7.

Corollary 5.3.3. Let $\phi: M \rightarrow N$ be an $\mathscr{O}_{X, x}$-module homomorphism. Then:

a) $\phi: M \rightarrow N$ is an $\mathscr{O}_{X, x}$-monomorphism of modules if and only if $\phi_{D}: M_{D} \rightarrow N_{D}$ is an $\mathscr{O}_{X, x}$-double monomorphism;

b) $\phi: M \rightarrow N$ is an $\mathscr{O}_{X, x^{-}}$epimorphism of modules if and only if $\phi_{D}: M_{D} \rightarrow N_{D}$ is an $\mathscr{O}_{X, x^{-}}$ double epimorphism;

c) $\phi: M \rightarrow N$ is an $\mathscr{O}_{X, x^{-}}$-isomorphism of modules if and only if $\phi_{D}: M_{D} \rightarrow N_{D}$ is an $\mathscr{O}_{X, x^{-}}$ double isomorphism. 
Here it is reasonable to emphasize the difference between the notions of injective homomorphism and monomorphism of modules, which are not the samething in the category of modules, since we are understanding the term monomorphism in the categorical sense, i.e, there is a left-inverse morphism.

The same remark has to be done between surjective homomorphism and epimorphism.

Remark 5.3.4. The covariant functor

$$
\begin{array}{cccc}
\mathbf{D}: \mathscr{T}\left(\mathscr{O}_{X, x}\right) & \longrightarrow & \mathscr{T}\left(\mathscr{O}_{X \times X,(x, x)}\right) \\
M & \longmapsto & M_{D} \\
\phi: M \rightarrow N & \longmapsto \phi_{D}: M_{D} \rightarrow N_{D}
\end{array}
$$

is not an isomorphism of categories anymore.

In fact, suppose $(X, x)$ irreducible. It is proved in 2.1.17 that the generic rank of the double of every module $M$ in $\mathscr{T}\left(\mathscr{O}_{X, x}\right)$ has generic rank even. Thus, the map between the objects cannot be surjective.

\subsection{The double in a quotient of a free $\mathscr{O}_{X}$-module of finite rank}

Let $W$ be an $\mathscr{O}_{X, x}$-submodule of $\mathscr{O}_{X, x}^{p}$ and consider the quotient map

$$
\pi: \mathscr{O}_{X, x}^{p} \longrightarrow \frac{\mathscr{O}_{X, x}^{p}}{W}
$$

Then, $W_{D}$ is an $\mathscr{O}_{X \times X,(x, x)}$-submodule of $\mathscr{O}_{X \times X,(x, x)}^{2 p}$.

Let $h \in \mathscr{O}_{X, x}^{p}$. We define the double of $h+W \in \frac{\mathscr{O}_{X, x}^{p}}{W}$ as

$$
(h+W)_{D}:=h_{D}+W_{D} \in \frac{\mathscr{O}_{X \times X,(x, x)}^{2 p}}{W_{D}} .
$$

Notice the definition of $(h+W)_{D}$ does not depend of the choice of the representative $h$. In fact, if $h+W=g+W$ then $h-g \in W \Longrightarrow(h-g)_{D} \in W_{D} \Longrightarrow h_{D}-g_{D} \in W_{D} \Longrightarrow h_{D}+W_{D}=$ $g_{D}+W_{D}$

Now, we want to define the double of a submodule $M$ of $\frac{\mathscr{O}_{X, x}^{p}}{W}$. We have that $\pi^{-1}(M)$ is a submodule of $\mathscr{O}_{X, x}^{p}$ and $\pi^{-1}(M) \supset W$, hence $\left(\pi^{-1}(M)\right)_{D}$ is a submodule of $\mathscr{O}_{X \times X,(x, x)}^{2 p}$ which contains $W_{D}$.

Then, we define the double of $M$ as

$$
M_{D}:=\frac{\left(\pi^{-1}(M)\right)_{D}}{W_{D}}
$$


which is an $\mathscr{O}_{X \times X,(x, x)}$-submodule of $\frac{\mathscr{O}_{X \times X,(x, x)}^{2 p}}{W_{D}}$.

If we call $\tilde{M}:=\pi^{-1}(M)$ then

$$
M=\frac{\tilde{M}}{W} \text { and } M_{D}=\frac{\tilde{M}_{D}}{W_{D}} .
$$

Rewriting with standard notation, we conclude that, if $M$ is a submodule of $\mathscr{O}_{X, x}^{p}$ and $M \supset W$ then

$$
\left(\frac{M}{W}\right)_{D}=\frac{M_{D}}{W_{D}}
$$

and is generated by $\left\{(h+W)_{D} \mid h \in M\right\}$.

\subsection{The double homomorphism relative to an analytic map germ}

Let $(Y, y)$ and $(X, x)$ be germs of analytic spaces, and let $\varphi:(Y, y) \rightarrow(X, x)$ be an analytic map germ. The pullback map $\varphi^{*}: \mathscr{O}_{X, x} \rightarrow \mathscr{O}_{Y, y}$ is a ring homomorphism, which induces an $\mathscr{O}_{X, x}$-algebra structure in $\mathscr{O}_{Y, y}$. Thus, every $\mathscr{O}_{Y, y}$-module is also an $\mathscr{O}_{X, x}$-module through this ring homomorphism.

We see that there is a natural $\mathscr{O}_{X \times X,(x, x)}$-algebra structure in $\mathscr{O}_{Y \times Y,(y, y)}$ induced by the pullback of $\varphi$. In fact, let

$$
\mu_{X, x}: \mathscr{O}_{X, x} \underset{\mathbb{C}}{\otimes} \mathscr{O}_{X, x} \longrightarrow \mathscr{O}_{X \times X,(x, x)}
$$

be the $\mathbb{C}$-algebra homomorphism such that $\mu_{X, x}(f \underset{\mathbb{C}}{\otimes} g)$ is the germ of the map

$$
\begin{aligned}
U \times U & \rightarrow \mathbb{C} \\
(u, v) & \mapsto f(u) \cdot g(v)
\end{aligned}
$$

and let

$$
\mu_{Y, y}: \mathscr{O}_{Y, y} \underset{\mathbb{C}}{\otimes} \mathscr{O}_{Y, y} \longrightarrow \mathscr{O}_{Y \times Y,(y, y)}
$$

the same for $(Y, y)$.

Since $\varphi^{*}: \mathscr{O}_{X, x} \rightarrow \mathscr{O}_{Y, y}$ is a ring homomorphism then we have a natural $\mathbb{C}$-algebra homomorphism

$$
\varphi^{\otimes}: \mathscr{O}_{X, x} \underset{\mathbb{C}}{\otimes} \mathscr{O}_{X, x} \longrightarrow \mathscr{O}_{Y, y} \underset{\mathbb{C}}{\otimes} \mathscr{O}_{Y, y}
$$

such that $\varphi^{\otimes}(f \underset{\mathbb{C}}{\otimes} g)=\varphi^{*}(f) \underset{\mathbb{C}}{\otimes} \varphi^{*}(g), \forall f, g \in \mathscr{O}_{X, x}$. In fact, the map

$$
\begin{aligned}
\mathscr{O}_{X, x} \times \mathscr{O}_{X, x} & \longrightarrow \mathscr{O}_{Y, y} \underset{\mathbb{C}}{\otimes} \mathscr{O}_{Y, y} \\
(f, g) & \longmapsto\left(\varphi^{*}(f)\right) \underset{\mathbb{C}}{\underset{\mathbb{N}}{\otimes}\left(\varphi^{*}(g)\right)}
\end{aligned}
$$


is $\mathbb{C}$-bilinear. So, the existence and uniqueness of $\varphi^{\otimes}$ is provided by the universal property of the tensor product. It is known that $\mu_{X, x}$ and $\mu_{Y, y}$ are $\mathbb{C}$-algebra isomorphisms, so we can consider the $\mathbb{C}$-algebra homomorphism $\varepsilon_{\varphi}: \mathscr{O}_{X \times X,(x, x)} \rightarrow \mathscr{O}_{Y \times Y,(y, y)}$ such that the following diagram is commutative:

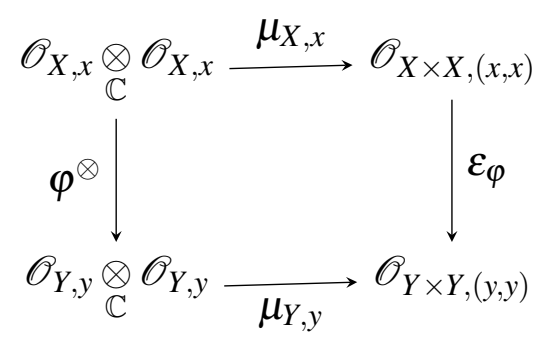

Since $\mu_{X, x}$ and $\mu_{Y, y}$ are $\mathbb{C}$-algebra isomorphisms then we can identify $\varepsilon_{\varphi} \cong \varphi^{\otimes}$, and $\varphi^{\otimes}: \mathscr{O}_{X \times X,(x, x)} \rightarrow \mathscr{O}_{Y \times Y,(y, y)}$ induces in $\mathscr{O}_{Y \times Y,(y, y)}$ an $\mathscr{O}_{X \times X,(x, x)}$-algebra structure.

Lemma 5.5.1. Let $\alpha \in \mathscr{O}_{X \times X,(x, x)}$. Suppose that $U$ is an open subset of $X$ containing $x$ where a representative of $\alpha$ is defined on $U \times U$. For each $w \in U$ let $\alpha^{w} \in \mathscr{O}_{X, x}$ be the germ of the map

$$
\begin{aligned}
\alpha^{w}: U & \rightarrow \mathbb{C} \\
z & \mapsto \alpha(z, w)
\end{aligned}
$$

For each $y_{2} \in \varphi^{-1}(U)$ let $\left(\varphi^{\otimes}(\alpha)\right)^{y_{2}} \in \mathscr{O}_{Y, y}$ be the germ of the map

$$
\begin{array}{rlcc}
\left(\varphi^{\otimes}(\alpha)\right)^{y_{2}}: \varphi^{-1}(U) & \rightarrow & \mathbb{C} \\
y_{1} & \mapsto & \left(\varphi^{\otimes}(\alpha)\right)\left(y_{1}, y_{2}\right)
\end{array}
$$

Then

$$
\varphi^{*}\left(\alpha^{\varphi\left(y_{2}\right)}\right)=\left(\varphi^{\otimes}(\alpha)\right)^{y_{2}}, \forall y_{2} \in \varphi^{-1}(U)
$$

Proof. We can write $\alpha=\sum_{i}\left(f_{i} \underset{\mathbb{C}}{\otimes} g_{i}\right)$, with $f_{i}, g_{i} \in \mathscr{O}_{X, x}$. For all $y_{1} \in \varphi^{-1}(U)$ we have:

$$
\begin{aligned}
& \varphi^{*}\left(\alpha^{\varphi\left(y_{2}\right)}\right)\left(y_{1}\right)=\alpha^{\varphi\left(y_{2}\right)}\left(\varphi\left(y_{1}\right)\right)=\alpha\left(\varphi\left(y_{1}\right), \varphi\left(y_{2}\right)\right) \sum_{i}\left(f_{i}\left(\varphi\left(y_{1}\right)\right) \underset{\mathbb{C}}{\otimes} g_{i}\left(\varphi\left(y_{2}\right)\right)\right) \\
& =\left(\sum_{i}\left(\varphi^{*}\left(f_{i}\right)\right) \underset{\mathbb{C}}{\otimes}\left(\left(\varphi^{*}\left(g_{i}\right)\right)\right)\left(y_{1}, y_{2}\right)=\left(\varphi^{\otimes}(\alpha)\right)\left(y_{1}, y_{2}\right)=\left(\varphi^{\otimes}(\alpha)\right)^{y_{2}}\left(y_{1}\right),\right. \text { and the lemma }
\end{aligned}
$$
is proved.

Clearly we get the analogous result if we fix the first coordinate instead the second one. Consider the projections $\pi_{1}^{X}, \pi_{2}^{X}: X \times X \rightarrow X$ and $\pi_{1}^{Y}, \pi_{2}^{Y}: Y \times Y \rightarrow Y$. 
Theorem 5.5.2. Let $M \subseteq \mathscr{O}_{X, x}^{p}$ and $N \subseteq \mathscr{O}_{Y, y}^{q}$ be submodules. If $\phi: M \rightarrow N$ is an $\mathscr{O}_{X, x}$-module homomorphism then there exists a unique $\mathscr{O}_{X \times X,(x, x)}$-module homomorphism $\phi_{D, \varphi}=\phi_{D}: M_{D} \rightarrow$ $N_{D}$ such that the following diagram is commutative:

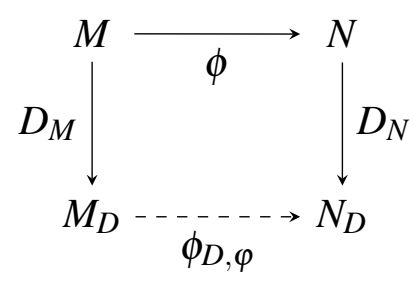

The map $\phi_{D, \varphi}=\phi_{D}$ is called the double of $\phi$ relative to $\varphi:(\mathbf{Y}, \mathbf{y}) \rightarrow(\mathbf{X}, \mathbf{x})$.

Proof. Since $M_{D}$ is generated by $\left\{h_{D} \mid h \in M\right\}$ then we can define $\phi_{D}: M_{D} \rightarrow N_{D}$ in a natural way: for each $u=\sum_{i} \alpha_{i}\left(h_{i}\right)_{D}$ with $\alpha_{i} \in \mathscr{O}_{X \times X,(x, x)}$ and $h_{i} \in M$ we define

$$
\phi_{D}(u):=\sum_{i} \alpha_{i}\left(\phi\left(h_{i}\right)\right)_{D}=\sum_{i} \varphi^{\otimes}\left(\alpha_{i}\right)\left(\phi\left(h_{i}\right)\right)_{D}
$$

which belongs to $N_{D}$.

Claim : $\phi_{\mathbf{D}}$ is well defined. In fact, suppose that $\sum_{i} \alpha_{i}\left(h_{i}\right)_{D}=\sum_{j} \beta_{j}\left(g_{j}\right)_{D}$, with $\alpha_{i}, \beta_{j} \in$ $\mathscr{O}_{X \times X,(x, x)}$ and $h_{i}, g_{j} \in M$. So, we obtain two equations:

$$
\begin{aligned}
& \sum_{i} \alpha_{i}\left(h_{i} \circ \pi_{1}^{X}\right)=\sum_{j} \beta_{j}\left(g_{j} \circ \pi_{1}^{X}\right) \\
& \sum_{i} \alpha_{i}\left(h_{i} \circ \pi_{2}^{X}\right)=\sum_{j} \beta_{j}\left(g_{j} \circ \pi_{2}^{X}\right) .
\end{aligned}
$$

Take $U$ an open neighborhood of $x$ in $X$ where representatives of $\alpha_{i}, \beta_{j}$ are defined on $U \times U$, and representatives of $h_{i}, g_{j}$ are defined on $U$. For each $w \in U$ define $\alpha_{i}^{w}, \beta_{j}^{w} \in \mathscr{O}_{X, x}$ given by the germs of the maps

$$
\begin{aligned}
& \alpha_{i}^{w}: U \longrightarrow \mathbb{C} \quad \beta_{j}^{w}: U \longrightarrow \mathbb{C} \\
& z \longmapsto \alpha_{i}(z, w) \quad z \longrightarrow \beta_{j}(z, w)
\end{aligned}
$$

The equation (1) implies that $\sum_{i} \alpha_{i}^{w} h_{i}=\sum_{j} \beta_{j}^{w} g_{j}, \forall w \in U$. Applying $\phi$ (which is a $\mathscr{O}_{X, x^{-}}$ homomorphism) in both sides of the last equation we get

$$
\sum_{i} \alpha_{i}^{w} \phi\left(h_{i}\right)=\sum_{j} \beta_{j}^{w} \phi\left(g_{j}\right), \forall w \in U
$$

By the $\mathscr{O}_{X, x}$-module structure on $N$ induced by $\varphi^{*}$, the last equation boils down to

$$
\sum_{i} \varphi^{*}\left(\alpha_{i}^{w}\right) \phi\left(h_{i}\right)=\sum_{j} \varphi^{*}\left(\beta_{j}^{w}\right) \phi\left(g_{j}\right), \forall w \in U
$$


By Lemma 5.5.1 we conclude that

$$
\sum_{i}\left(\varphi^{\otimes}\left(\alpha_{i}\right)\right)^{y_{2}} \phi\left(h_{i}\right)=\sum_{j}\left(\varphi^{\otimes}\left(\beta_{j}\right)\right)^{y_{2}} \phi\left(g_{j}\right), \forall y_{2} \in \varphi^{-1}(U) .
$$

Hence,

$$
\sum_{i} \varphi^{\otimes}\left(\alpha_{i}\right)\left(\phi\left(h_{i}\right) \circ \pi_{1}^{Y}\right)=\sum_{j} \varphi^{\otimes}\left(\beta_{j}\right)\left(\phi\left(g_{j}\right) \circ \pi_{1}^{Y}\right)
$$

Working with the analogous result of the Lemma 5.5.1, the equation (2) implies that

$$
\sum_{i} \varphi^{\otimes}\left(\alpha_{i}\right)\left(\phi\left(h_{i}\right) \circ \pi_{2}^{Y}\right)=\sum_{j} \varphi^{\otimes}\left(\beta_{j}\right)\left(\phi\left(g_{j}\right) \circ \pi_{2}^{Y}\right) .
$$

Therefore,

$$
\sum_{i} \varphi^{\otimes}\left(\alpha_{i}\right)\left(\phi\left(h_{i}\right)\right)_{D}=\sum_{j} \varphi^{\otimes}\left(\beta_{j}\right)\left(\phi\left(g_{j}\right)\right)_{D}
$$

and $\phi_{D}$ is well-defined.

Now, by the definition of $\phi_{D}$, it is clear that $\phi_{D}$ is an $\mathscr{O}_{X \times X,(x, x)}$-module homomorphism and is the unique satisfying the property $\phi_{D}\left(h_{D}\right)=(\phi(h))_{D}, \forall h \in M$, i.e,

$$
\phi_{D}\left(h \circ \pi_{1}^{X}, h \circ \pi_{2}^{X}\right)=\left(\phi(h) \circ \pi_{1}^{Y}, \phi(h) \circ \pi_{2}^{Y}\right) .
$$

Notice that this approach generalizes what we have defined in Section 5.1, taking $\varphi:(X, x) \rightarrow(X, x)$ as the identity map. The main motivation of this approach is the fact that when we work with integral closure of modules, the analytic curves $\varphi:(\mathbb{C}, 0) \rightarrow(X, x)$ has a key role.

Clearly the Propositions 5.1.5, 5.1.6 (a,c) and the Corollary 5.1.7 (a,b,d) still hold for the double homomorphism relative to an analytic map.

We can write the Proposition 5.1.6 (b) on this new language as follows:

Proposition 5.5.3. Let $\varphi:(Y, y) \rightarrow(X, x)$ and $\varphi^{\prime}:(Z, z) \rightarrow(Y, y)$ be analytic map germs, $M \subseteq$ $\mathscr{O}_{X, x}^{p}, N \subseteq \mathscr{O}_{Y, y}^{q}$ and $P \subseteq \mathscr{O}_{Z, z}^{r}$ submodules. Let $\phi: M \rightarrow N$ be an $\mathscr{O}_{X, x}$-module homomorphism and $\phi^{\prime}: N \rightarrow P$ be an $\mathscr{O}_{Y, y}$-module homomorphism. Then, $\phi^{\prime} \circ \phi: M \rightarrow P$ is an $\mathscr{O}_{X, x}$-module homomorphism, considering $P$ with the $\mathscr{O}_{X, x}$-module structure induced by the pullback of $\varphi \circ \varphi^{\prime}:(Z, z) \rightarrow(X, x)$ and

$$
\left(\phi^{\prime} \circ \phi\right)_{D, \varphi \circ \varphi^{\prime}}=\phi_{D, \varphi^{\prime}}^{\prime} \circ \phi_{D, \varphi}
$$

Proof. For all $\alpha \in \mathscr{O}_{X, x}$ and $h \in M$, working with the module structures induced by the pullbacks of the analytic map germs, we have: 


$$
\phi^{\prime} \circ \phi(\alpha h)=\phi^{\prime}(\alpha \phi(h))=\phi^{\prime}\left(\varphi^{*}(\alpha) \phi(h)\right)=\varphi^{*}(\alpha) \phi^{\prime}(\phi(h))=\varphi^{*}\left(\varphi^{*}(\alpha)\right)\left(\phi^{\prime} \circ \phi(h)\right)=
$$

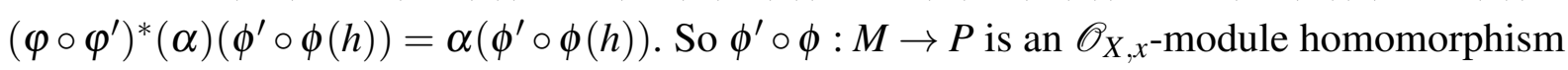
and $\left(\phi^{\prime} \circ \phi\right)_{D, \varphi \circ \varphi^{\prime}}$ is well defined and clearly is equal to $\phi_{D, \varphi^{\prime}}^{\prime} \circ \phi_{D, \varphi}$. 

[1] W. Adkins, Weak normality and Lipschitz saturation for ordinary singularities, Compositio Math. 51, 149-157, (1984). No citation.

[2] D. A. H. Ament, J. J. Nuño-Ballesteros, B. Oréfice-Okamoto and J. N. Tomazella, The Euler obstruction of a function on a determinantal variety and on a curve. Bull. Braz. Math. Soc. (N.S.) 47, no. 3, 955-970, (2016). Citation on pages 45 and 109.

[3] L. Birbrair, Local bi-Lipschitz classification of 2-dimensional semialgebraic sets. Houston J. Math. 25, no. 3, 453-472, (1999). Citation on page 24.

[4] L. Birbrair, Metric theory of singularities. Lipschitz geometry of singular spaces, Singularities in geometry and topology, World Sci. Publ., Hackensack, NJ, 223-233, (2007). Citation on page 24 .

[5] L. Birbrair, Lipschitz geometry of curves and surfaces definable in $\mathscr{O}$-minimal structures, Illinois J. Math. 52, no. 4, 1325-1353, (2008). Citation on page 24.

[6] L. Birbrair, J. C. F. Costa, A. Fernandes and M. A. S. Ruas, $\mathscr{K}$-bi-Lipschitz equivalence of real function-germs, Proc. Amer. Math. Soc. 135, no. 4, 1089-1095, (2007). Citation on page 24 .

[7] L. Birbrair and A. Fernandes, Local Lipschitz geometry of real weighted homogeneous surfaces, Geom. Dedicata 135, 211-217, (2008). Citation on page 24.

[8] L. Birbrair, A. Fernandes and V. Grandjean, On the bi-Lipschitz contact equivalence of plane complex function germs, J. Singul. 13, 1-10, (2015). Citation on page 24.

[9] L. Birbrair, A. Fernandes, V. Grandjean and D. O'Shea, Choking horns in Lipschitz geometry of complex algebraic varieties, Appendix by Walter D. Neumann. J. Geom. Anal. 24, no. 4, 1971-1981, (2014). Citation on page 24.

[10] L. Birbrair, A. Fernandes, D. Lê and J. Sampaio, Lipschitz regular complex algebraic sets are smooth, Proc. Amer. Math. Soc. 144, no. 3, 983-987, (2016). Citation on page 24.

[11] L. Birbrair, A. Fernandes and W. Neumann, Bi-Lipschitz geometry of complex surface singularities, Geom. Dedicata. 139, 259-267, (2009). Citation on page 24. 
[12] L. Birbrair, A. Fernandes and W. Neumann, Bi-Lipschitz geometry of weighted homogeneous surface singularities, Math. Ann. 342, no. 1, 139-144, (2008). Citation on page 24.

[13] L. Birbrair, A. Fernandes and D. Panazzolo, Lipschitz classification of functions on a Hölder triangle, Algebra i Analiz 20, no. 5, 1-8 (2008); translation in St. Petersburg Math. J. 20, no. 5, 681-686, (2008). Citation on page 24.

[14] L. Birbrair, W. Neumann and A. Pichon, The thick-thin decomposition and the bilipschitz classification of normal singularities, Acta Math. 212, no. 2, 199-256, (2014). Citation on page 24 .

[15] C. Bivià-Ausina, The integral closure of ideals in $\mathbb{C}\{x, y\}$, Comm. Algebra 31, no. 12, 6115-6134, (2003). Citation on page 27.

[16] C. Bivià-Ausina, The integral closure of modules, Buchsbaum-Rim multiplicities and Newton polyhedra, J. London Math. Soc. (2) 69, no. 2, 407-427, (2004). Citation on page 27.

[17] J. W. Bruce, Families of symmetric matrices, Moscow Math. J., 3, no 2, 335-360, (2003). Citation on page 45 .

[18] W. Bruns and U. Vetter, Determinantal Rings, Springer- Verlang, New York, (1998). Citation on page 44 .

[19] D. A. Buchsbaum and D. S. Rim, A generalized Koszul complex. II. Depth and multiplicity, Trans. AMS 111, 197-224, (1963). Citation on page 35.

[20] G. Comte, Multiplicity of complex analytic sets and bi-Lipschitz maps, in: Real analytic and Algebraic Singularities, Nagoya/Sapporo/Hachioji, 1996. Pitman Res. Notes Math. Ser., Longman, Harlow, 182-188, (1998). Citation on page 24.

[21] J. C. F. Costa, A note on topological contact equivalent, Real and Complex singularities, London Math. Soc. Lecture Notes Series 380, 114-124, (2010). Citation on page 49.

[22] J. C. F. Costa, T. Nishimura and M. A. S. Ruas, Bi-Lipschitz $\mathscr{A}$-equivalence of $\mathscr{K}$ equivalent map germs, Rev. R. Acad. Cienc. Exactas Fís. Nat. Ser. A Math. RACSAM 108, no. 1, 173-182, (2014). Citation on page 24.

[23] J. C. F. Costa, M. J. Saia and C. H. Soares Júnior, Bi-Lipschitz $\mathscr{G}$-triviality and Newton polyhedra, $\mathscr{G}=\mathscr{R}, \mathscr{C}, \mathscr{K}, \mathscr{R}_{V}, \mathscr{C}_{V}, \mathscr{K}_{V}$. Real and complex singularities, Contemp. Math., 569, Amer. Math. Soc., Providence, RI, 29-43, (2012). Citation on page 24.

[24] J. C. F. Costa, M. J. Saia and C. H. Soares Júnior, Bi-Lipschitz $\mathscr{A}$-triviality of map germs and Newton filtrations, Topology Appl. 159, no. 2, 430-436, (2012). Citation on page 24. 
[25] T. da Silva, Categorical Aspects of the Double Structure, arXiv:1704.08664 [math.AG], (2017). No citation.

[26] T. da Silva, N. G. Grulha Jr. and M. Pereira, The Bi-Lipschitz Equisingularity of Essentially Isolated Determinantal Singularities, arXiv:1705.07180 [math.AG], (2017) (Accepted for publication in Bulletin of the Brazilian Mathematical Society, New Series (67)). Citation on page 24 .

[27] J. Damon, The unfolding and determinancy theorems for subgoups of $\mathscr{A}$ and $\mathscr{K}$, Memoirs of the American Mathematical Society, Providence RI, (1984). Citation on page 45.

[28] Z. Denkowska, S. Lojasiewicz and J. Stasica, Certaines propriétés elementaires des ensembles sous-analytiques, Bull. Acad. Polon. Sci. 27, 530-536, (1979). Citation on page 48.

[29] J. Damon and B. Pike, Solvable groups, free divisors and nonisolated matrix singularities II: Vanishing topology. Geom. Topol. 18, no. 2, 911-962, (2014). Citation on page 45.

[30] W. Ebeling and S. M. Gusein-Zade, On indices of 1-forms on determinantal singularities, Proc. Steklov Inst. Math. 267, no. 1, 113-124, (2009). Citation on page 46.

[31] D. Eisenbud, Commutative Algebra with a View Toward Algebraic Geometry, Springer, Graduate Texts in Mathematics, (2004). Citation on page 31.

[32] A. Fernandes, Topological equivalence of complex curves and bi-Lipschitz homeomorphisms, Michigan Math. J. 51, no. 3, 593-606, (2003). Citation on page 24.

[33] A. Fernandes and M. A. S. Ruas, Bi-Lipschitz determinacy of quasihomogeneous germs, Glasg. Math. J. 46, no. 1, 77-82, (2004). Citation on page 24.

[34] A. Fernandes and M. A. S. Ruas, Rigidity of bi-Lipschitz equivalence of weighted homogeneous function-germs in the plane. Proc. Amer. Math. Soc. 141, no. 4, 1125-1133, (2013). Citation on page 24 .

[35] A. Fernandes and J. Sampaio, Multiplicity of analytic hypersurface singularities under bi-Lipschitz homeomorphisms, J. Topol. 9, no. 3, 927-933, (2016). Citation on page 24.

[36] H. Fitting, Die Determinantenideale einer Modulo Jahresler, Jahresber. Dtsch. Math. Ver. 46, 195-220, (1936). Citation on page 33.

[37] A. Frühbis-Krüger and A. Neumer, Simple Cohen-Macaulay Codimension 2 Singularities, Communications in Algebra, 38:2, 454-495, (2010). Citation on pages 25, 45, 104, 105, 106, 110, and 111.

[38] A. Frühbis-Krüger, Classification of Simple Space Curves Singularities, Communications in Algebra, 27 (8), pp. 3993-4013, (1999). Citation on pages 45 and 46. 
[39] T. Gaffney, The genericity of the infinitesimal Lipschitz condition for hypersurfaces, J. Singul. 10, 108-123, (2014). Citation on pages 24, 42, 43, 44, 45, 51, 56, 66, 70, 71, 72, $77,78,79,92$, and 108 .

[40] T. Gaffney, Bi-Lipschitz equivalence, integral closure and invariants, Proceedings of the 10th International Workshop on Real and Complex Singularities. Edited by: M. Manoel, Universidade de São Paulo, M. C. Romero Fuster, Universitat de Valencia, Spain, C. T. C. Wall, University of Liverpool, London Mathematical Society Lecture Note Series (No.380), 125-137, (2010). Citation on pages 24, 42, 51, 82, 89, and 103.

[41] T. Gaffney, Integral Closure of Modules and Whitney equisingularity, Invent. Math. 107, 301-322, (1992). Citation on pages 24, 32, 33, 34, 40, 67, and 97.

[42] T. Gaffney, Notes in Equisingularity and the Theory of Integral Closure, Brazil-Mexico 2nd Meeting on Singularities, (2015). Citation on pages 35, 36, 39, and 40.

[43] T. Gaffney, Generalized Buchsbaum-Rim Multiplicities and a Theorem of Rees, Communications in Algebra, 31, 3811-3828 (2003). Citation on pages 65 and 78.

[44] T. Gaffney, Equisingularity of Plane Sections, $t^{1}$ Condition, and the Integral Closure of Modules, Real and Complex Singularities, ed. by W. L. Marar, Pitman Research Series in Math. 333, Longman, (1995). Citation on page 76.

[45] T. Gaffney, The Multiplicity Polar Theorem, arxiv:math/0703650v1 [math.CV], (2007). Citation on page 79.

[46] T. Gaffney and R. Gassler, Segre numbers and hypersurface singularities, J. Algebraic Geometry 8, 695-736, (1999). Citation on page 103.

[47] T. Gaffney, N. G. Grulha Jr. and M. A. S. Ruas, The local Euler obstruction and topology of the stabilization of associated determinantal varieties, arXiv:1611.00749 [math.AG], (2017). Citation on page 45.

[48] T. Gaffney and S. Kleiman, Specialization of integral dependence for modules, Inventiones mathematicae. 137, 541-574 (1999). Citation on pages 34, 35, 72, 77, 82, 88, and 101.

[49] T. Gaffney and M. Vitulli, Weak subintegral closure of ideals, Adv. Math. 226, no. 3, 2089-2117, (2011). No citation.

[50] C. G. Gibson, Singular points of smooth mappings, Research Notes in Mathematics 25, (1979). Citation on page 49.

[51] G. M. Greuel and J. Steenbrink, On the Topology of Smoothable Singularities, Proceedings of Symposia in Pure Mathematics, 40, Part 1, 535- 545, (1983). No citation. 
[52] A. Grothendieck, Éléments de géométrie algébrique, IV, Publications de l'IHES, PUF, (1964). Citation on page 31.

[53] R. C. Gunning and H. Rossi, Analytic Functions of Several Complex Variables, AMS Chelsea Publishing, vol. 368, (1965). No citation.

[54] J.-P. Henry and M. Merle, Limites de normales, conditions de Whitney et éclatement d'Hironaka, Proceedings of Symposia in Pure Mathematics, Volume 40, Arcata 1981Singularities, Part 2, American Mathematical Society, Providence, RI, 575-584, (1983). Citation on page 23.

[55] J. Henry and A. Parusiński, Invariants of bi-Lipschitz equivalence of real analytic functions, Geometric singularity theory, Banach Center Publ., 65, Polish Acad. Sci. Inst. Math., Warsaw, 67-75, (2004). Citation on page 24.

[56] J. Henry and A. Parusiński, Existence of moduli for bi-Lipschitz equivalence of analytic functions, Compositio Math. 136, no. 2, 217-235, (2003). No citation.

[57] D. Juniati and G. Valette, Bi-Lipschitz trivial quasi-homogeneous stratifications, Saitama Math. J. 26 (2009), 1-13, (2010). Citation on page 24.

[58] D. Juniati and D. Trotman, Determination of Lipschitz stratification for the surfaces $y^{a}=$ $z^{b} x^{c}+x^{d}$, Singularités Franco-Japonaises, Sémin. Congr., 10, Soc. Math., France, Paris, 127-138, (2005). Citation on page 24.

[59] D. Juniati, D. Trotman and G. Valette, Lipschitz stratifications and generic wings, J. London Math. Soc. (2) 68, no. 1, 133-147, (2003). Citation on pages 24 and 49.

[60] L. Kovalev, Bi-Lipschitz embedding of projective metrics, Conform. Geom. Dyn. 18, 110118, (2014). Citation on page 24.

[61] S. Koike and L. Paunescu, The directional dimension of subanalytic sets is invariant under bi-Lipschitz homeomorphisms, Ann. Inst. Fourier (Grenoble) 59, no. 6, 2445-2467, (2009). Citation on page 24.

[62] S. Kleiman and A. Thorup, A geometric theory of the Buchsbaum-Rim multiplicity, J. Algebra 167, 168-231, (1994). Citation on pages 35, 57, and 79.

[63] D. Lê and B. Teissier, Cycles evanescents, sections planes et conditions de Whitney, II. (French) [Vanishing cycles, plane sections and Whitney conditions. II] Singularities, Part 2 (Arcata, Calif., 1981) Proc. Symp. Pure. Math., 40, Amer. Math. Soc., Providence, RI, 65-103, (1983). No citation.

[64] M. Lejeune-Jalabert and B. Teissier, Clôture intégrale des idéaux et équisingularité. (French) [Integral closure of ideals and equisingularity] With an appendix by Jean-Jacques 
Risler. Ann. Fac. Sci. Toulouse Math. (6) 17, no. 4, 781-859 (2008). Citation on pages 28, $30,31,32,41$, and 75 .

[65] J. Lipman, Absolute saturation of one-dimensional local rings, Amer. J. Math. 97, 771-790, (1975). Citation on page 23.

[66] J. Lipman, Relative Lipschitz Saturation, Amer. J. Math. 97, no. 3, 791-813, (1975). Citation on pages 23 and 41.

[67] S. Lojasiewicz, Ensembles semi-analytiques, IHES, (1965). Citation on page 48.

[68] E.J.N. Looijenga, Isolated singular points on complete intersection, Lond. Math. Soc. Lect. Note Ser., vol 77, Cambridge University Press, (1984). Citation on page 33.

[69] J. Mather, Notes on topological stability, Harvard, (1970). Citation on page 38.

[70] T. Mostowski, A criterion for Lipschitz equisingularity. Bull. Polish Acad. Sci. Math. 37, no. 1-6, 109-116 (1989). Citation on pages 23, 24, 46, 49, and 103.

[71] T. Mostowski, Tangent cones and Lipschitz stratifications. Singularities (Warsaw, 1985), 303-322, Banach Center Publ., 20, PWN, Warsaw, (1988). Citation on pages 23 and 24.

[72] T. Mostowski, Lipschitz stratifications, Dissertationes Math. PWN, Warszawa, (1985). Citation on pages 23, 24, 47, and 49.

[73] T. Mostowski, Lipschitz stratifications and Lipschitz isotopies, Geometric singularity theory, Banach Center Publ., 65, Polish Acad. Sci. Inst. Math., Warsaw, 179-210, (2004). Citation on page 24 .

[74] W. Neumann and A. Pichon, Lipschitz geometry of complex surfaces, J. Singul. 10, 225-234, (2014). Citation on page 24.

[75] N. Nguyen and G. Valette, Lipschitz stratifications in $\mathscr{O}$-minimal structures, Ann. Sci. Éc. Norm. Supér. (4) 49, no. 2, 399-421, (2016). No citation.

[76] J. J. Nuño-Ballesteros, B. Oréfice-Okamoto and J. N. Tomazella, The vanishing Euler characteristic of an isolated determinantal singularity, Israel J. Math. 197, no. 1, 475-495 (2013). Citation on pages 45 and 109.

[77] D. O'Shea, The bilipschitz geometry of the $A_{k}$ surface singularities, J. Singul. 12, 156-163, (2015). Citation on page 24.

[78] A. Parusiński, Lipschitz stratification of real analytic sets. Singularities (Warsaw, 1985), 323-333, Banach Center Publ., 20, PWN, Warsaw, (1988). Citation on pages 23, 24, and 49. 
[79] A. Parusiński, Lipschitz properties of semi-analytic sets. Ann. Inst. Fourier (Grenoble) 38, no. 4, 189-213, (1988). Citation on pages 23 and 24.

[80] A. Parusiński, Lipschitz Stratifications, Global Analysis in modern mathematics; a symposium in honor of Richard Palais' sixtieth birthday, 73-89, (1993). Citation on pages 24 and 48 .

[81] A. Parusiński, Bi-Lipschitz trivialization of the distance function to a stratum of a stratification, Ann. Polon. Math. 87, 213-218, (2005). Citation on page 24.

[82] A. Parusiński, Lipschitz stratifications of subanalytic sets, Ann. Sci. École Norm. Sup. (4) 27, no. 6, 661-696, (1994). Citation on pages 23, 24, and 49.

[83] M. S. Pereira, Variedades Determinantais e Singularidades de Matrizes, Ph.D. Thesis, ICMC-USP, http://www.teses.usp.br/teses/disponiveis/55/55135/tde-22062010133339/en.php (2010). Citation on page 45.

[84] F. Pham, Fractions lipschitziennes et saturation de Zariski des algèbres analytiques complexes. Exposé d'un travail fait avec Bernard Teissier. Fractions lipschitziennes d'une algèbre analytique complexe et saturation de Zariski, Centre Math. lÈcole Polytech., Paris, 1969. Actes du Congrès International des Mathématiciens (Nice, 1970), Tome 2, pp. 649654. Gauthier-Villars, Paris, (1971). Citation on pages 23 and 24.

[85] F. Pham and B. Teissier, Fractions lipschitziennes d'une algébre analytique complexe et saturation de Zariski, Centre de Mathématiques de l'Ecole Polytechnique (Paris), http://people.math.jussieu.fr/teissier/old-papers.html, (1969). Citation on pages 23, 24, and 40.

[86] D. Rees, a-transforms of local rings and a theorem on multiplicities of ideals, Proceedings Camb. Philos., 57, 1, 8-17, (1961). Citation on page 31.

[87] D. Rees, Reduction of Modules. Math. Proc. Camb. Philos. Soc. 101, 431-449 (1987). Citation on pages 33 and 34.

[88] J. Rotman, An Introduction to Homological Algebra, 2nd edition, Springer, (2009). No citation.

[89] M. Ruas and M. Pereira, Codimension two determinantal varieties with isolated singularities. Math. Scand. 115, no. 2, 161-172, (2014). Citation on pages 45 and 103.

[90] J. Risler and D. Trotman, Bi-Lipschitz invariance of the multiplicity, Bull. London Math. Soc. 29, no. 2, 200-204, (1997). No citation.

[91] M. A. S. Ruas and G. Vallete, $C^{0}$ and bi-Lipschitz $\mathscr{K}$-equivalence of mappings, Math. Z. 269, no. 1-2, 293-308, (2011). Citation on page 24. 
[92] J. Sampaio, Bi-Lipschitz homeomorphic subanalytic sets have bi-Lipschitz homeomorphic tangent cones, Selecta Math. (N. S.) 22, no. 2, 553-559, (2016). Citation on page 24.

[93] M. Schaps, Deformations of Cohen-Macaulay schemes of codimension 2 and nonsingular deformations of space curves, Amer. J. Math. 99, 669-684, (1977). Citation on page 45.

[94] L. Siebenmann and D. Sullivan, On complexes that are Lipschitz manifolds, Proceedings of the Georgia Topology conference, Athens, Ga., (1977), Geometric topology, Academic Press, New York-London, 503-525, (1979). No citation.

[95] I. Swanson and C. Huneke, Integral Closure of Ideals, Rings and Modules, London Mathematical Society, Lecture Note Series 336, (2006). Citation on pages 27, 28, 29, and 30.

[96] F. Tari, Singularidades de aplicações diferenciáveis, Notas Didáticas do Instituto de Ciências Matemáticas e Computação - USP, (1999). Citation on page 49.

[97] B. Teissier, The hunting of invariants in the geometry of discriminants, Real and complex singularities (Proc. Ninth Nordic Summer School/NAVF Sympos. Math., Oslo, 1976), Sijthoff and Noordhoff, Alphen aan den Rijn, 565-678, (1977). No citation.

[98] B. Teissier, Multiplicités polaires, sections planes, et conditions de Whitney, in "Proc. La Rábida, 1981." J. M. Aroca, R. Buchweitz, M. Giusti and M. Merle (eds.) Springer Lecture Notes in Math. 961, 314-491, (1982). Citation on pages 23 and 39.

[99] R. Thom, Ensembles et morphismes stratifiés, Bull. Amer. Math. Soc. 75, 240-284, (1969). Citation on page 23.

[100] G. N. Tjurina, Absolute isolatedness of rational singularities and triple rational points., Func. Anal. Appl. 2: 324-333, (1968). Citation on page 105.

[101] D. Trotman, Bi-Lipschitz equisingularity, Real and complex singularities, London Math. Soc. Lecture Note Ser., 380, Cambridge Univ. Press, Cambridge, 338-349, (2010). Citation on pages 23,24 , and 49 .

[102] G. Valette, A bilipschitz version of Hardt's theorem, C. R. Acad. Sci. Paris, Sér. I, t. 340, 895-900, (2005). Citation on page 24.

[103] G. Valette, Lipschitz triangulations, Illinois J. Math. 49, no. 3, 953-979, (2005). Citation on page 24 .

[104] G. Valette, Bi-Lipschitz sufficiency of jets, J. Geom. Anal. 19, no. 4, 963-993, (2009). Citation on page 24.

[105] G. Valette, Multiplicity $\bmod 2$ as a metric invariant, Discrete and Computational Geometry, 43, 668-679, (2010). Citation on page 24. 
[106] G. Valette, On bi-Lipschitz stability of families of functions. J. Singul. 6, 179-198, (2012). Citation on page 24.

[107] J.-L. Verdier, Stratifications de Whitney et théorème de Bertini-Sard, Inventiones Math. 36, 295-312, (1976). No citation.

[108] J. Wahl, Smoothings of normal surface singularities, Topology, 20, 219- 246, (1981). No citation.

[109] H. Whitney, Local properties of analytic varieties Differential and Combinatorial Topology (edited by Stewart S. Cairns), (A Symposium in Honor of Marston Morse), Princeton Univ. Press, Princeton, NJ, 205-244, (1965). Citation on pages 23 and 39.

[110] H. Whitney, Tangents to an analytic variety, Ann. of Math. (2) 81, 496-549, (1965). Citation on pages 23 and 39.

[111] O. Zariski, General Theory of saturation and of saturated Local Rings, I. Saturation of complete local domains of dimension one having arbitrary coefficient fields (of characteristic zero). Amer. J. Math. 93, 573-648, (1971). Citation on pages 23 and 24.

[112] O. Zariski, General Theory of saturation and of saturated Local Rings, II. Saturated local rings of dimension 1, Amer. J. Math. 93, 872-964, (1971). Citation on pages 23 and 24.

[113] O. Zariski, General theory of saturation and of saturated local rings, III. Saturation in arbitrary dimension and, in particular, saturation of algebroid hypersurfaces, Amer. J. Math. 97, 415-502, (1975). Citation on pages 23 and 24.

[114] X. Zhang, Chern-Schwartz-MacPherson Class of Determinantal Varieties, arXiv:1605.05380 [math.AG], (2016). Citation on page 45. 

(w)-regularity, 40

Bi-Lipschitz equisingularity on determinantal varieties, 108

Chain homotopy, 123

Chain of points, 48

Determinantal Varieties, 44

Double chain complex, 122

Double homomorphism, 114, 129

Double of a module, 51

Double of an ideal, 42

EIDS, 46

Genericity of the $i L_{A}$ condition, 72

Infinitesimal Lipschitz conditions, 43, 67

Integral closure of ideals, 27

Integral closure of modules, 32

Lipschitz equisingularity, 47

Lipschitz saturation of a module, 81, 83, 86, 90

Lipschitz saturation of an ideal, 41

Lipschitz stratifications, 48

Multiplicity of a pair of modules, 35

Reduction of ideals, 30

Simple isolated Cohen-Macaulay singularities, 104

Stratifications, 47

The Double Category, 125

The generic rank of the double of a module, 59

Whitney equisingularity, 37 


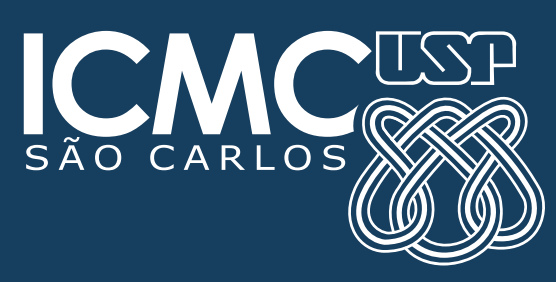

